\title{
Iron-Catalyzed $\alpha$-Alkylation of Ketones with Secondary Alcohols: Access to $\beta$-Disubstituted Carbonyl Compounds
}

\author{
Léo Bettoni $^{[a]}$, Sylvain Gaillard ${ }^{[a]}$, Jean-Luc Renaud*[a]
}

aNormandie Univ, LCMT, ENSICAEN, UNICAEN, CNRS, 6 boulevard du Maréchal Juin, 14000 Caen, France.

Supporting Information

\section{Table Of Contents}

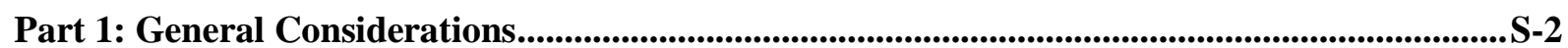

Part 2: Alkylation of 1-mesitylethan-1-one with 2-phenylethanol ...................................................S-3

Part 3: General procedure for the alkylation of ketones .........................................................................S-4

Part 4: Functionalization of $\beta$-disubstituted carbonyl compounds.....................................................S-19

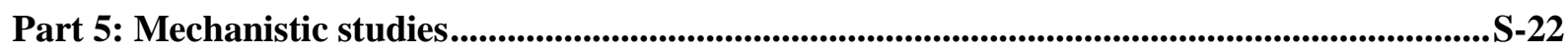

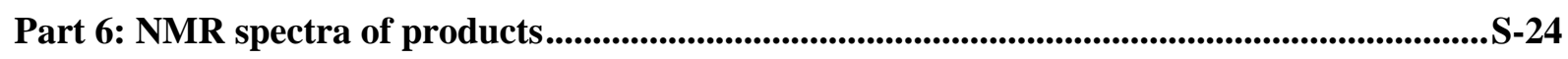

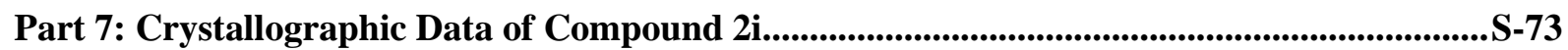




\section{Part 1: General Considerations}

All air- and moisture-sensitive manipulations were carried out using standard vacuum line Schlenk tubes techniques. Dry toluene was dried using a solvent purification system from Innovative Technologies, by passage through towers containing activated alumina. Xylene was purchased from Carlo Reba and was distillated over sodium and stocked over $4 \AA$ molecular sieves. Both and dioxane, dimethyl sulfoxide, dimethyl carbonate, tert-butanol and alcohol substrat were deglazed prior to use by bubbling argon gas directly in the solvent. Other solvents and chemicals were purchased from different suppliers and used as received. Neutral alumina was purchased from Alfa Aesar (Brockmann Grade I, 58 Angstroms, -60 Mesh

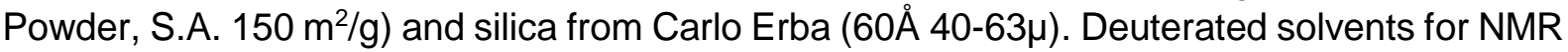
spectroscopy were purchased from Sigma Aldrich and used as received. NMR spectra were recorded on a $500 \mathrm{MHz}$ Brücker spectrometer. Proton $\left({ }^{1} \mathrm{H}\right)$ NMR information is given in the following format: multiplicity (s, singlet; $d$, doublet; $t$, triplet; q, quartet; $m$, multiplet), coupling constant(s) $(J)$ in Hertz $(\mathrm{Hz})$, number of protons, type. The prefix app is occasionally applied when the true signal multiplicity was unresolved and $b r$ indicates the signal in question broadened. Carbon $\left({ }^{13} \mathrm{C}\right)$ NMR spectra are reported in ppm $(\delta)$ relative to $\mathrm{CDCl}_{3}$ unless noted otherwise. Infrared spectra were recorded over a PerkinElmer Spectrum 100 FT-IR Spectrometer using neat conditions. HRMS (ESI-TOF) $\mathrm{m} / \mathrm{z}$ analyses were performed by Laboratoire de Chimie Moléculaire et Thioorganique analytical Facilities. 


\section{Part 2: Alkylation of 1-mesitylethan-1-one with 2-phenylethanol}

Table S1: Optimization of the reaction conditions ${ }^{\mathrm{a}}$
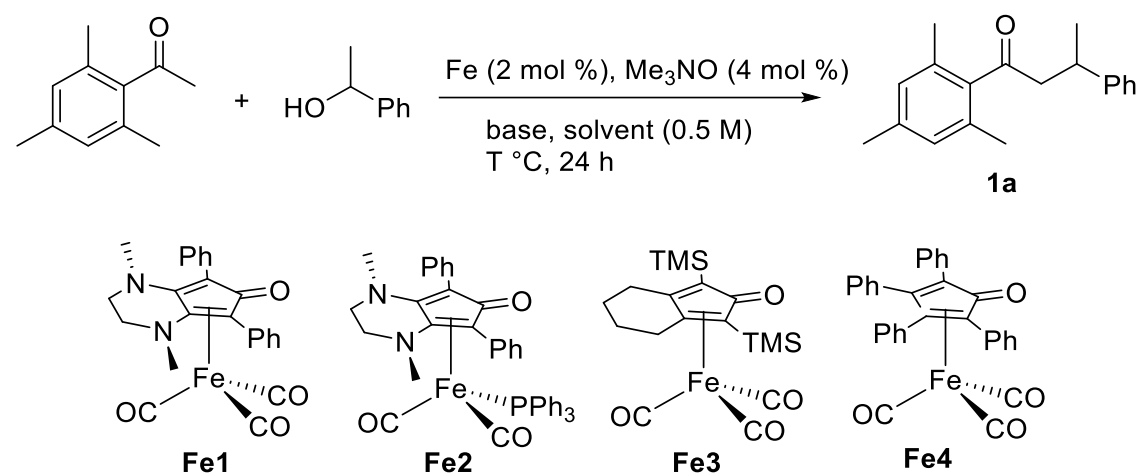

\begin{tabular}{|c|c|c|c|c|c|c|}
\hline entry & $\begin{array}{l}\text { alcohol } \\
\text { (equiv) }\end{array}$ & catalyst & $\begin{array}{c}\text { temperature } \\
\left({ }^{\circ} \mathrm{C}\right)\end{array}$ & base (equiv) & solvent & $1 \mathrm{a}(\%)$ \\
\hline 1 & 2 & $\mathrm{Fe} 1$ & 90 & $\mathrm{~K}_{3} \mathrm{PO}_{4}(1)$ & toluene & - \\
\hline 2 & 2 & $\mathrm{Fe} 1$ & 90 & $\mathrm{NaOMe}(1)$ & toluene & - \\
\hline 3 & 2 & Fe1 & 90 & $\mathrm{NaO}^{t} \mathrm{Bu}(1)$ & toluene & 11 \\
\hline 4 & 2 & $\mathrm{Fe} 1$ & 110 & $\mathrm{NaOMe}(1)$ & toluene & 32 \\
\hline 5 & 2 & $\mathrm{Fe} 1$ & 110 & $\mathrm{~K}_{3} \mathrm{PO}_{4}(1)$ & toluene & 10 \\
\hline 6 & 2 & $\mathrm{Fe} 1$ & 110 & $\mathrm{NaO}^{\mathrm{t}} \mathrm{Bu}(1)$ & toluene & 98 \\
\hline 7 & 2 & $\mathrm{Fe} 1$ & 110 & $\mathrm{NaOH}(1)$ & toluene & 48 \\
\hline 8 & 2 & $\mathrm{Fe} 1$ & 110 & $\mathrm{Cs}_{2} \mathrm{CO}_{3}(0.1)$ & toluene & - \\
\hline 9 & 2 & $\mathrm{Fe} 1$ & 110 & $\mathrm{Cs}_{2} \mathrm{CO}_{3}(1)$ & toluene & 61 \\
\hline 10 & 1.5 & Fe1 & 110 & $\mathrm{NaO}^{t} \mathrm{Bu}(1)$ & toluene & 98 \\
\hline 11 & 1.5 & $\mathrm{Fe} 1$ & 110 & $\mathrm{NaO}(\mathrm{Bu}(1)$ & CPME & 76 \\
\hline 12 & 1.5 & $\mathrm{Fe} 1$ & 110 & $\mathrm{NaO}(\mathrm{Bu}(1)$ & tBuOH & 79 \\
\hline 13 & 1.5 & $\mathrm{Fe} 2$ & 110 & $\mathrm{NaO}(\mathrm{Bu}(1)$ & toluene & 78 \\
\hline 14 & 1.5 & $\mathrm{Fe} 3$ & 110 & $\mathrm{NaO}(\mathrm{Bu}(1)$ & toluene & 80 \\
\hline 15 & 1.5 & $\mathrm{Fe} 4$ & 110 & $\mathrm{NaO}(\mathrm{Bu}(1)$ & toluene & 84 \\
\hline
\end{tabular}

[a] Reaction performed using $0.5 \mathrm{mmol}$ of starting material, conversion was determined by ${ }^{1} \mathrm{H}-\mathrm{NMR}$ analysis of the crude mixture. 


\section{Part 3: General procedure for the alkylation of ketones}

\section{General Procedure:}

In a $15 \mathrm{~mL}$ flame-dried Schlenk tube equipped with a stirring bar, 1-mesitylethan-1-one (0.5 $\mathrm{mmol}, 83 \mu \mathrm{L}$ ), the desired alcohol (1.5 equiv), $\mathrm{Me}_{3} \mathrm{NO}$ (2.2 $\mathrm{mg}, 2 \mathrm{~mol} \%$ ), iron complex Fe1 (4.56 mg, $2 \mathrm{~mol} \%$ ), NaO'Bu (48 mg, 1 equiv) and toluene $(1.0 \mathrm{~mL})$ were poured in under an argon atmosphere. The mixture was rapidly stirred at room temperature for 2 min and then placed into a pre-heated oil bath at $110^{\circ} \mathrm{C}$ and stirred over 24 hours. The mixture was cooleddown to room temperature, filtrated over silica with diethyl ether and concentrated under reduced pressure. The conversion was determined by ${ }^{1} \mathrm{H}$-NMR spectroscopy, and then, the residue was purified by flash chromatography on silica gel using pentane-diethyl ether as eluent to afford the desired product.

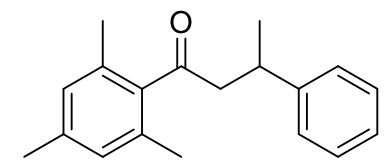

Chemical Formula: $\mathrm{C}_{19} \mathrm{H}_{22} \mathrm{O}$

\section{- 1-mesityl-3-phenylbutan-1-one' (1a)}

According to general procedure, alkylation of 1-mesitylethan-1-one $(0.5 \mathrm{mmol})$ with 1 phenylethan-1-ol (1.5 equiv, $90 \mu \mathrm{L}$ ) afforded the pure product 1a as a light yellow oil $(98 \mathrm{mg}$, $87 \%$ ) by silica flash column chromatography (pentane/diethyl ether 98:2). ${ }^{1} \mathbf{H}-\mathbf{N M R}\left(\mathbf{C D C l}_{3}\right.$, $500 \mathrm{MHz}$ ): $\delta$ 7.31-7.24 (m, 4H), 7.21-7.18 (m, 1H), $6.80(\mathrm{~s}, 2 \mathrm{H}), 3.50$ (sext, $J=7.0 \mathrm{~Hz}, 1 \mathrm{H}$ ), $3.02\left(\mathrm{ABX}, J_{A X}=6.0 \mathrm{~Hz} ; J_{A B}=18.4 \mathrm{~Hz}, 1 \mathrm{H}\right), 2.95\left(\mathrm{ABX}, J_{B X}=7.7 \mathrm{~Hz} ; J_{B A}=18.4 \mathrm{~Hz}, 1 \mathrm{H}\right), 2.27$ (s, 3H), 2.07 (s, 6H), 1.37 (d, $J=7.0 \mathrm{~Hz}, 3 \mathrm{H}$ ) ppm. ${ }^{13} \mathbf{C}-\mathbf{N M R}\left(\mathrm{CDCl}_{3}, 125 \mathbf{~ M H z}\right): \delta$ 209.0, 146.5, 139.5, 138.3, 132.6, 128.5 (2C), 128.4 (2C), 127.0 (2C), 126.2 (2C), 53.0, 34.5, 22.3, 21.0, $18.9(2 \mathrm{C}) \mathrm{ppm}$.

\section{- Scale up of the reaction to $5 \mathrm{mmol}$ starting material}

In a $30 \mathrm{~mL}$ flame-dried Schlenk tube equipped with a stirring bar 1-mesitylethan-1-one (5 mmol, $830 \mu \mathrm{L}$ ), the desired alcohol (1.5 equiv, $900 \mu \mathrm{L}$ ), $\mathrm{Me}_{3} \mathrm{NO}$ (22.2 $\mathrm{mg}, 2 \mathrm{~mol} \%$ ), iron complex Fe1 (45.6 mg, $2 \mathrm{~mol} \%$ ), NaO'Bu (480 mg, 1 equiv) and toluene $(10 \mathrm{~mL})$ were poured in under an argon atmosphere. The mixture was rapidly stirred at room temperature for 2 min and then placed into a pre-heated oil bath at $110^{\circ} \mathrm{C}$ and stirred over 24 hours. The mixture was cooleddown to room temperature, filtrated over silica with diethyl ether and concentrated under reduced pressure. Then the residue was purified by flash chromatography on silica gel using pentane-diethyl ether (98:2) as eluent to afford the pure product 1a as a light yellow oil (1.104 g, $83 \%)$. ${ }^{1} \mathrm{H}-\mathrm{NMR}$ data was comparable with the previous NMR data.

\footnotetext{
${ }^{1}$ Charkraborty, P.; Kumar Gangwar, M.; Emayavaramban, B.; Manoury, E.; Poli, R.; Sundararaju, B. Chem. Sus. Chem, 2019, 12, 3463-3467.
} 
<smiles>Cc1c(C)c(C)c(C(=O)CC(C)c2ccccc2)c(C)c1C</smiles>

Chemical Formula: $\mathrm{C}_{21} \mathrm{H}_{26} \mathrm{O}$

\section{- 1-(2,3,4,5,6-pentamethylphenyl)-3-phenylbutan-1-one ${ }^{1}(2 a)$}

According to general procedure, alkylation of 1-(2,3,4,5,6-pentamethylphenyl)ethan-1-one (0.5 $\mathrm{mmol}, 95 \mathrm{mg}$ ) with 1-phenylethan-1-ol (1.5 equiv, $90 \mu \mathrm{L}$ ) afforded the pure product $2 \mathrm{a}$ as a white solid (119 mg, $81 \%$ ) by silica flash column chromatography (pentane/diethyl ether 98:2). ${ }^{1} \mathrm{H}-\mathrm{NMR}\left(\mathrm{CDCl}_{3}, \mathbf{5 0 0} \mathbf{~ M H z}\right): \delta$ 7.30-7.25 (m, 4H), 7.21-7.17 (m, $\left.1 \mathrm{H}\right), 3.52$ (sext, $J=6.9 \mathrm{~Hz}$, $1 \mathrm{H}), 3.00\left(\mathrm{ABX}, J_{A X}=5.7 \mathrm{~Hz} ; J_{A B}=18.9 \mathrm{~Hz}, 1 \mathrm{H}\right), 2.92\left(\mathrm{ABX}, J_{B X}=7.7 \mathrm{~Hz} ; J_{B A}=18.9 \mathrm{~Hz}, 1 \mathrm{H}\right)$, 2.21 (s, 3H), 2.15 (s, 6H), 1.99 (br. s, 6H), 1.38 (d, J=7.0 Hz, 3H) ppm. ${ }^{13}$ C-NMR $\left(\mathbf{C D C l}_{3}, 125\right.$ MHz): $\delta 210.1,146.6,140.4,135.3,133.0$ (2C), 128.4 (2C), 127.3, 127.0 (2C), 126.1 (2C), 53.7, 34.1, 22.3, 16.8, 16.6, 15.8 (2C) ppm.<smiles>CC(C)c1cc(C(C)C)c(C(=O)CC(C)c2ccccc2)c(C(C)C)c1</smiles>

Chemical Formula: $\mathrm{C}_{25} \mathrm{H}_{34} \mathrm{O}$

\section{- 1-(2,3,4,5,6-pentamethylphenyl)-3-phenylbutan-1-one (3a)}

According to general procedure, alkylation of 1-(2,4,6-triisopropylphenyl)ethan-1-one $(0.5$ $\mathrm{mmol}, 123 \mathrm{mg}$ ) with 1-phenylethan-1-ol (1.5 equiv, $90 \mu \mathrm{L}$ ) afforded the pure product $3 \mathrm{a}$ as a colorless oil (127 mg, $73 \%$ ) by silica flash column chromatography (pentane/diethyl ether 98:2). ${ }^{1} \mathrm{H}-\mathrm{NMR}\left(\mathrm{CDCl}_{3}, \mathbf{5 0 0} \mathbf{M H z}\right): \delta$ 7.29-7.23 (m, 4H), 7.19-7.14 (m, 1H), 6.92 (br. s, 2H), 3.55 (sext, $J=6.9 \mathrm{~Hz}, 1 \mathrm{H}), 3.02\left(\mathrm{ABX}, J_{A X}=6.7 \mathrm{~Hz} ; J_{A B}=18.8 \mathrm{~Hz}, 1 \mathrm{H}\right), 2.88\left(\mathrm{ABX}, J_{B X}=6.8\right.$ $\mathrm{Hz} ; J_{B A}=18.9 \mathrm{~Hz}, 1 \mathrm{H}$ ), 2.79 (sept, $J=6.9 \mathrm{~Hz}, 1 \mathrm{H}$ ), 2.70-2.65 (m, 1H), 2.11 (br. s, $\left.1 \mathrm{H}\right), 1.35$ (d, $J=7.0 \mathrm{~Hz}, 3 \mathrm{H}), 1.27-0.94(\mathrm{~m}, 18 \mathrm{H})$ ppm. ${ }^{13} \mathbf{C}-\mathbf{N M R}\left(\mathbf{C D C l}_{3}, 125 \mathbf{~ M H z}\right): \delta 209.2,149.4,146.4$, 143.5, 137.8, 128.4 (2C), 127.1 (2C), 126.2, 120.9, 54.8, 34.3, 34.2, 30.6, 24.9, 23.9 (2C), 21.9 ppm. IR (neat): v 2960, 2928, 2870, 1699, 1605, 1570, 1493, 1458, 1403, 1363, 1282, 1217, 1154, 1103, 987, 876, 759, 699, $551 \mathrm{~cm}^{-1}$. HRMS (ESI-TOF) $\mathbf{m} / \mathbf{z}[\mathbf{M}+\mathbf{H}]^{+}$Calcd for $\mathrm{C}_{25} \mathrm{H}_{35} \mathrm{O} 335.2688$; Found: 335.2691.<smiles>COc1ccc(C(C)CC(=O)c2c(C)cc(C)cc2C)cc1</smiles>

Chemical Formula: $\mathrm{C}_{20} \mathrm{H}_{24} \mathrm{O}_{2}$

\section{- 1-mesityl-3-(4-methoxyphenyl)butan-1-one (1b)}

According to general procedure, alkylation of 1-mesitylethan-1-one $(0.5 \mathrm{mmol})$ with 1-(4methoxyphenyl)ethan-1-ol (1.5 equiv, $114 \mathrm{mg}$ ) afforded the pure product $\mathbf{1 b}$ as a colorless oil (129 mg, $87 \%$ ) by silica flash column chromatography (pentane/diethyl ether 98:2). ${ }^{1} \mathrm{H}-\mathrm{NMR}$ $\left(\mathrm{CDCl}_{3}, 500 \mathrm{MHz}\right): \delta 7.17(\mathrm{~d}, J=8.5 \mathrm{~Hz}, 2 \mathrm{H}), 6.84(\mathrm{~d}, J=8.5 \mathrm{~Hz}, 2 \mathrm{H}), 6.81(\mathrm{~s}, 2 \mathrm{H}), 3.80$ (s, $3 \mathrm{H}$ ), 3.48 (sext, $J=6.8 \mathrm{~Hz}, 1 \mathrm{H}), 3.00\left(\mathrm{ABX}, J_{A X}=6.2 \mathrm{~Hz} ; J_{A B}=18.3 \mathrm{~Hz}, 1 \mathrm{H}\right), 2.93\left(\mathrm{ABX}, J_{B X}=\right.$ $\left.7.5 \mathrm{~Hz} ; J_{B A}=18.3 \mathrm{~Hz}, 1 \mathrm{H}\right), 2.28(\mathrm{~s}, 3 \mathrm{H}), 2.09(\mathrm{~s}, 6 \mathrm{H}), 1.35(\mathrm{~d}, J=6.9 \mathrm{~Hz}, 3 \mathrm{H})$ ppm. ${ }^{13} \mathrm{C}-\mathrm{NMR}$ ( $\mathrm{CDCl}_{3}, 125 \mathrm{MHz}$ ): $\delta$ 209.2, 158.1, 139.6, 138.7, 138.3, 132.7 (2C), 128.6 (2C), 128.0 (2C), 113.9 (2C), 55.3, 53.4, 33.8, 22.6, 21.1, 19.0 (2C) ppm. IR (neat): v 2959, 2922, 2835, 1698, 
1610, 1512, 1455, 1458, 1302, 1244, 1037, 986, 829, 600, $542 \mathrm{~cm}^{-1}$. HRMS (ESI-TOF) m/z: $[\mathrm{M}+\mathrm{Na}]^{+}$Calcd for $\mathrm{C}_{20} \mathrm{H}_{24} \mathrm{O}_{2} \mathrm{Na} 319.1674 ;$ Found: 319.1667 .<smiles>Cc1cc(C)c(C(=O)CC(C)c2ccc(C(F)(F)F)cc2)c(C)c1</smiles>

Chemical Formula: $\mathrm{C}_{20} \mathrm{H}_{21} \mathrm{~F}_{3} \mathrm{O}$

\section{- 1-mesityl-3-(4-(trifluoromethyl)phenyl)butan-1-one (1c)}

According to general procedure, alkylation of 1-mesitylethan-1-one $(0.5 \mathrm{mmol})$ with 1-(4trifluoromethylphenyl)ethan-1-ol (1.5 equiv, $143 \mathrm{mg}$ ) afforded the pure product $1 \mathrm{c}$ as a colorless oil (123 $\mathrm{mg}, 74 \%$ ) by silica flash column chromatography (pentane/diethyl ether 98:2). ${ }^{1} \mathrm{H}-\mathrm{NMR}\left(\mathrm{CDCl}_{3}, 500 \mathrm{MHz}\right): \delta 7.55(\mathrm{~d}, J=8.0 \mathrm{~Hz}, 2 \mathrm{H}), 7.38(\mathrm{~d}, J=8.0 \mathrm{~Hz}, 2 \mathrm{H}), 6.81$ (s, 2H), 3.58 (sext, $J=6.9 \mathrm{~Hz}, 1 \mathrm{H}$ ), $3.03\left(\mathrm{ABX}, J_{A X}=6.5 \mathrm{~Hz} ; J_{A B}=18.5 \mathrm{~Hz}, 1 \mathrm{H}\right), 2.97\left(\mathrm{ABX}, J_{B X}=\right.$ $\left.7.2 \mathrm{~Hz} ; J_{B A}=18.5 \mathrm{~Hz}, 1 \mathrm{H}\right), 2.27(\mathrm{~s}, 3 \mathrm{H}), 2.07(\mathrm{~s}, 6 \mathrm{H}), 1.38(\mathrm{~d}, J=6.9 \mathrm{~Hz}, 3 \mathrm{H})$ ppm. ${ }^{19} \mathrm{~F}-\mathrm{NMR}$ $\left(\mathrm{CDCl}_{3}, \mathbf{5 0 0} \mathbf{M H z}\right): \delta-62.3$ ppm. ${ }^{13} \mathrm{C}-\mathrm{NMR}\left(\mathrm{CDCl}_{3}, 125 \mathrm{MHz}\right): \delta 208.4,150.5,139.2,138.5$, 132.5 (2C), 128.6 (q, J=32.1 Hz), 128.5 (2C), 127.5 (2C), 125.3 (q, J=3.8 Hz, 2C), 122.1 (d, $J=270 \mathrm{~Hz}$ ), 52.7, 34.4, 22.0, 20.9, 18.9 (2C) ppm. IR (neat): $v$ 2965, 2926, 1698, 1611, 1456, 1323, 1162, 1114, 1067, 1016, 840, $602 \mathrm{~cm}^{-1}$. HRMS (ESI-TOF) $\mathbf{m} / \mathbf{z}:$ [M + H] ${ }^{+}$Calcd for $\mathrm{C}_{20} \mathrm{H}_{22} \mathrm{OF}_{3}$ 335.1623; Found 335.1619.

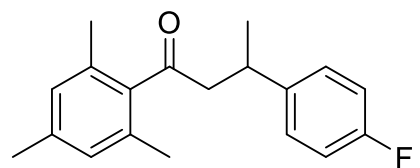

Chemical Formula: $\mathrm{C}_{19} \mathrm{H}_{21} \mathrm{FO}$

\section{- 3-(4-fluorophenyl)-1-mesitylbutan-1-one (1d)}

According to general procedure, alkylation of 1-mesitylethan-1-one $(0.5 \mathrm{mmol})$ with 1-(4fluorophenyl)ethan-1-ol (1.5 equiv, $105 \mathrm{mg}$ ) afforded the pure product $\mathbf{1 d}$ as a light yellow oil (98 $\mathrm{mg}, 69 \%$ ) by silica flash column chromatography (pentane/diethyl ether 98:2). ${ }^{1} \mathrm{H}-\mathrm{NMR}$ $\left(\mathrm{CDCl}_{3}, 500 \mathrm{MHz}\right): \delta 7.23-7.20(\mathrm{~m}, 2 \mathrm{H}), 6.96(\mathrm{t}, J=8.7 \mathrm{~Hz}, 2 \mathrm{H}), 6.80(\mathrm{~s}, 2 \mathrm{H}), 3.50$ (sext, $J=$ $7.0 \mathrm{~Hz}, 1 \mathrm{H}), 2.98\left(\mathrm{ABX}, J_{A X}=6.8 \mathrm{~Hz} ; J_{A B}=18.5 \mathrm{~Hz}, 1 \mathrm{H}\right), 2.91\left(\mathrm{ABX}, J_{B X}=7.2 \mathrm{~Hz} ; J_{B A}=18.5\right.$ $\mathrm{Hz}, 1 \mathrm{H}), 2.26(\mathrm{~s}, 3 \mathrm{H}), 2.05(\mathrm{~s}, 6 \mathrm{H}), 1.34(\mathrm{~d}, J=7.0 \mathrm{~Hz}, 3 \mathrm{H}) \mathrm{ppm} .{ }^{19} \mathrm{~F}-\mathrm{NMR}\left(\mathrm{CDCl}_{3}, 500 \mathbf{M H z}\right)$ : $\delta$-117.0 ppm. ${ }^{13} \mathbf{C}-N M R\left(\mathbf{C D C l}_{3}, \mathbf{1 2 5} \mathbf{~ M H z}\right): \delta 208.8,161.3$ (d, $\left.J=242 \mathrm{~Hz}\right), 142.0$ (d, $J=3.1$ $\mathrm{Hz}$ ), 139.3, 138.3, 132.5 (2C), $128.5(2 \mathrm{C}), 128.4$ (d, $J=7.8 \mathrm{~Hz}, 2 \mathrm{C}), 115.0$ (d, J=21 Hz, 2C), 53.2, 33.8, 22.4, 21.0, 18.9 (2C) ppm. IR (neat): v 2962, 2924, 1698, 1609, 1509, 1455, 1401, 1281, 1221, 1282, 1221, 1158, 1097, 986, 850, 833, 600, $538 \mathrm{~cm}^{-1}$. HRMS (ESI-TOF) m/z: $[\mathrm{M}+\mathrm{H}]^{+}$Calcd for $\mathrm{C}_{19} \mathrm{H}_{22} \mathrm{OF}$ 285.1650; Found 285.1655.<smiles>Cc1cc(C)c(C(=O)CC(C)c2ccc(Cl)cc2)c(C)c1</smiles>

Chemical Formula: $\mathrm{C}_{19} \mathrm{H}_{21} \mathrm{ClO}$

\section{- 3-(4-chlorophenyl)-1-mesitylbutan-1-one (1e)}

According to general procedure, alkylation of 1-mesitylethan-1-one $(0.5 \mathrm{mmol})$ with 1-(4chlorophenyl)ethan-1-ol (1.5 equiv, $117 \mathrm{mg}$ ) afforded the pure product $1 \mathrm{e}$ as a colorless oil (121 $\mathrm{mg}, 80 \%$ ) by silica flash column chromatography (pentane/diethyl ether 98:2). ${ }^{1} \mathbf{H}-\mathbf{N M R}$ 
$\left(\mathrm{CDCl}_{3}, 500 \mathrm{MHz}\right): \delta 7.16(\mathrm{~d}, J=8.2 \mathrm{~Hz}, 2 \mathrm{H}), 7.10(\mathrm{~d}, J=8.2 \mathrm{~Hz}, 2 \mathrm{H}), 6.71(\mathrm{~s}, 2 \mathrm{H}), 3.40$ (sext, $J=6.9 \mathrm{~Hz}, 1 \mathrm{H}), 2.89\left(\mathrm{ABX}, J_{A X}=6.4 \mathrm{~Hz} ; J_{A B}=18.4 \mathrm{~Hz}, 1 \mathrm{H}\right), 2.83\left(\mathrm{ABX}, J_{B X}=7.2 \mathrm{~Hz} ; J_{B A}=\right.$ $18.4 \mathrm{~Hz}, 1 \mathrm{H}), \delta 2.18(\mathrm{~s}, 3 \mathrm{H}), \delta 1.98(\mathrm{~s}, 6 \mathrm{H}), 1.25(\mathrm{~d}, J=7.0 \mathrm{~Hz}, 3 \mathrm{H})$ ppm. ${ }^{13} \mathrm{C}-\mathrm{NMR}\left(\mathrm{CDCl}_{3}\right.$, $125 \mathrm{MHz}): \delta 208.6,144.9,139.2,138.4,132.5$ (2C), 131.8, 128.5 (4C), 128.4 (2C), 52.9, 33.9, 22.2, 21.0, 18.9 (2C) ppm. IR (neat): v 2962, 2923, 1698, 1610, 1574, 1492, 1347, 1152, 1092 , 1013, 850, 826, 761, 600, $531 \mathrm{~cm}^{-1}$. HRMS (ESI-TOF) $\mathbf{m} / \mathbf{z}[\mathbf{M}+\mathbf{H}]^{+}$Calcd for $\mathrm{C}_{19} \mathrm{H}_{22} \mathrm{OCl} 335.1623$; Found 335.1619.<smiles>Cc1cc(C)c(C(=O)CC(C)c2ccc(Br)cc2)c(C)c1</smiles>

Chemical Formula: $\mathrm{C}_{19} \mathrm{H}_{21} \mathrm{BrO}$

\section{- 3-(4-bromophenyl)-1-mesitylbutan-1-one (1f)}

According to general procedure, alkylation of 1-mesitylethan-1-one $(0.5 \mathrm{mmol})$ with 1-(4bromophenyl)ethan-1-ol (1.5 equiv, $150 \mathrm{mg}$ ) afforded the pure product $1 \mathrm{f}$ as a colorless oil (132 mg, $77 \%$ ) by silica flash column chromatography (pentane/diethyl ether 98:2). ${ }^{1} \mathbf{H}-\mathbf{N M R}$ $\left(\mathrm{CDCl}_{3}, 500 \mathrm{MHz}\right): \delta 7.40(\mathrm{~d}, J=8.3 \mathrm{~Hz}, 2 \mathrm{H}), 7.13(\mathrm{~d}, J=8.3 \mathrm{~Hz}, 2 \mathrm{H}), 6.80(\mathrm{~s}, 2 \mathrm{H}), 3.47$ (sext, $J=6.9 \mathrm{~Hz}, 1 \mathrm{H}), 2.98\left(\mathrm{ABX}, J_{A X}=6.3 \mathrm{~Hz} ; J_{A B}=18.4 \mathrm{~Hz}, 1 \mathrm{H}\right), 2.92\left(\mathrm{ABX}, J_{B X}=7.3 \mathrm{~Hz} ; J_{B A}=\right.$ $18.4 \mathrm{~Hz}, 1 \mathrm{H}), \delta 2.27(\mathrm{~s}, 3 \mathrm{H}), \delta 2.07(\mathrm{~s}, 6 \mathrm{H}), 1.34(\mathrm{~d}, J=7.0 \mathrm{~Hz}, 3 \mathrm{H})$ ppm. ${ }^{13} \mathbf{C}-\mathbf{N M R}\left(\mathbf{C D C l}_{3}\right.$, $125 \mathrm{MHz}): \delta 208.6,145.4,139.2,138.4,132.5$ (2C), $131.5(2 \mathrm{C}), 128.8$ (2C), $128.5(2 \mathrm{C}), 119.9$, 52.8, 34.0, 22.1, 21.0, 18.9 (2C) ppm. IR (neat): v 2961, 2922, 1697, 1610, 1488, 1453, 1282 , 1152, 1009, 850, 821, 755, 597, $529 \mathrm{~cm}^{-1}$. HRMS (ESI-TOF) $\mathbf{m} / \mathbf{z}$ [M + Na] : Calcd for $\mathrm{C}_{19} \mathrm{H}_{21} \mathrm{OBrNa}$ 367.0673; Found 367.0675.<smiles>Cc1cc(C)c(C(=O)CC(C)c2ccc3ccccc3c2)c(C)c1</smiles>

Chemical Formula: $\mathrm{C}_{23} \mathrm{H}_{24} \mathrm{O}$

\section{- 1-mesityl-3-(naphthalen-2-yl)butan-1-one (1g)}

According to general procedure, alkylation of 1-mesitylethan-1-one $(0.5 \mathrm{mmol})$ with 1 (naphthalen-2-yl)ethan-1-ol (1.5 equiv, $129 \mathrm{mg}$ ) afforded the pure product $\mathbf{1 g}$ as a colorless oil (119 $\mathrm{mg}, 75 \%$ ) by silica flash column chromatography (pentane/diethyl ether 98:2). ${ }^{1} \mathbf{H}-\mathbf{N M R}$ $\left(\mathrm{CDCl}_{3}, 500 \mathrm{MHz}\right): \delta$ 7.83-7.80 (m, 3H), $\delta 7.70(\mathrm{~s}, 1 \mathrm{H}), 7.49-7.41(\mathrm{~m}, 3 \mathrm{H}), 6.82(\mathrm{~s}, 2 \mathrm{H}), 3.71$ (sext, $J=6.9 \mathrm{~Hz}, 1 \mathrm{H}), 3.15\left(\mathrm{ABX}, J_{A X}=6.0 \mathrm{~Hz} ; J_{A B}=18.4 \mathrm{~Hz}, 1 \mathrm{H}\right), 3.06\left(\mathrm{ABX}, J_{B X}=7.8 \mathrm{~Hz}\right.$; $\left.J_{B A}=18.4 \mathrm{~Hz}, 1 \mathrm{H}\right), \delta 2.29(\mathrm{~s}, 3 \mathrm{H}), \delta 2.10(\mathrm{~s}, 6 \mathrm{H}), 1.48(\mathrm{~d}, J=7.0 \mathrm{~Hz}, 3 \mathrm{H})$ ppm. ${ }^{13} \mathrm{C}-\mathrm{NMR}$ $\left(\mathrm{CDCl}_{3}, 125 \mathrm{MHz}\right): \delta 208.9,143.9,139.4,138.3,133.5,132.6$ (2C), 132.2, 128.5 (2C), 128.1, 127.6, 127.5, 125.9, 125.7, 125.3, 125.2, 52.9, 34.6, 22.3, 21.0, 19.0 (2C) ppm. IR (neat): v 2966, 2920, 1697, 1610, 1507, 1452, 1346, 1219, 1152, 1066, 1037, 852, 818, 746, 600, 477 $\mathrm{cm}^{-1}$. HRMS (ESI-TOF) m/z [M + H] ${ }^{+}$: Calcd for $\mathrm{C}_{23} \mathrm{H}_{25} \mathrm{O} 317.1905$; Found 317.1901. 


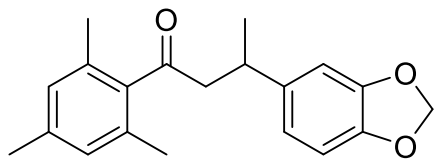

Chemical Formula: $\mathrm{C}_{20} \mathrm{H}_{22} \mathrm{O}_{3}$

\section{- 3-(benzo[d][1,3]dioxol-5-yl)-1-mesitylbutan-1-one (1h)}

According to general procedure, alkylation of 1-mesitylethan-1-one $(0.5 \mathrm{mmol})$ with 1 (benzo[d][1,3]dioxol-5-yl)ethan-1-ol (1.5 equiv, $125 \mathrm{mg}$ ) afforded the pure product $\mathbf{1 h}$ as a colorless oil (112 mg, $72 \%)$ by silica flash column chromatography (pentane/diethyl ether 95:5). ${ }^{1} \mathrm{H}-\mathrm{NMR}\left(\mathrm{CDCl}_{3}, 500 \mathrm{MHz}\right): \delta 6.79(\mathrm{~s}, 2 \mathrm{H}), 6.73-6.69(\mathrm{~m}, 3 \mathrm{H}), 5.91$ (s, 2H), 3.42 (sext, $J=6.9 \mathrm{~Hz}, 1 \mathrm{H}), 2.96\left(\mathrm{ABX}, J_{A X}=6.1 \mathrm{~Hz} ; J_{A B}=18.4 \mathrm{~Hz}, 1 \mathrm{H}\right), 2.88\left(\mathrm{ABX}, J_{B X}=7.6 \mathrm{~Hz} ; J_{B A}=\right.$ $18.4 \mathrm{~Hz}, 1 \mathrm{H}), 2.26(\mathrm{~s}, 3 \mathrm{H}), 2.08(\mathrm{~s}, 6 \mathrm{H}), 1.31(\mathrm{~d}, \mathrm{~J}=6.9 \mathrm{~Hz}, 3 \mathrm{H})$ ppm. ${ }^{13} \mathrm{C}-\mathrm{NMR}\left(\mathrm{CDCl}_{3}, 125\right.$ MHz): $\delta 208.9,147.6,145.8,140.5,139.4,138.3,132.6$ (2C), 128.5 (2C), 119.9, 108.2, 107.5, 100.8, 53.3, 34.3, 22.5, 21.0, 19.0 (2C) ppm. IR (neat): v 2960, 2921, 1697, 1610, 1504, 1486, 1439, 1373, 1234, 1153, 1038, 987, 850, 808, 639, $601 \mathrm{~cm}^{-1}$. HRMS (ESI-TOF) m/z [M + H]+: Calcd for $\mathrm{C}_{20} \mathrm{H}_{23} \mathrm{O}_{3} 311.1569$; Found 311.1573.

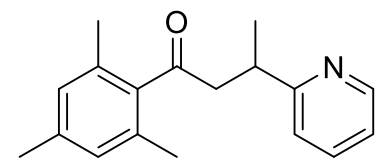

Chemical Formula: $\mathrm{C}_{18} \mathrm{H}_{21} \mathrm{NO}$

\section{- 1-mesityl-3-(pyridin-2-yl)butan-1-one (1i)}

According to general procedure, alkylation of 1-mesitylethan-1-one $(0.5 \mathrm{mmol})$ with 1-(pyridin2-yl)ethan-1-ol (1.5 equiv, $92 \mathrm{mg}$ ) afforded the pure product $1 \mathrm{i}$ as a light yellow oil $(64 \mathrm{mg}, 48$ $\%$ ) by silica flash column chromatography (pentane/diethyl ether 7:3). ${ }^{1} \mathbf{H}-\mathbf{N M R}\left(\mathbf{C D C l}_{3}, \mathbf{5 0 0}\right.$ MHz): $\delta 8.40$ (d, $J=4.4 \mathrm{~Hz}, 1 \mathrm{H}), 7.48(\mathrm{t}, J=7.6 \mathrm{~Hz}, 1 \mathrm{H}), 7.16(\mathrm{~d}, J=7.7 \mathrm{~Hz}, 1 \mathrm{H}), 7.00-6.98$ $(\mathrm{m}, 1 \mathrm{H}), 6.68(\mathrm{~s}, 2 \mathrm{H}), 3.50$ (sext, $J=6.9 \mathrm{~Hz}, 1 \mathrm{H}), 3.29\left(\mathrm{ABX}, J_{A X}=7.3 \mathrm{~Hz} ; J_{A B}=18.8 \mathrm{~Hz}, 1 \mathrm{H}\right)$, $2.86\left(\mathrm{ABX}, J_{B X}=6.2 \mathrm{~Hz} ; J_{B A}=18.8 \mathrm{~Hz}, 1 \mathrm{H}\right), 2.15(\mathrm{~s}, 3 \mathrm{H}), 1.99(\mathrm{~s}, 6 \mathrm{H}), 1.28(\mathrm{~d}, J=7.0 \mathrm{~Hz}, 3 \mathrm{H})$ ppm. ${ }^{13} \mathrm{C}-N M R\left(\mathbf{C D C l}_{3}, \mathbf{1 2 5} \mathbf{~ M H z}\right): \delta 209.2,164.8,149.0,139.3,138.0,136.3,132.4(2 \mathrm{C}), 128.3$ (2C), 122.6, 121.2, 51.0, 36.2, 21.0, 20.9, 18.8 (2C) ppm. IR (neat): v 2964, 2923, 1697, 1611, 1591, 1569, 1474, 1434, 1341, 1222, 1151, 992, 850, 748, 591, $534 \mathrm{~cm}^{-1}$. HRMS (ESI-TOF) $\mathbf{m} / \mathbf{z}[\mathbf{M}+\mathbf{H}]^{+}$: Calcd for $\mathrm{C}_{18} \mathrm{H}_{22} \mathrm{NO} 268.1701$; Found 268.1699.

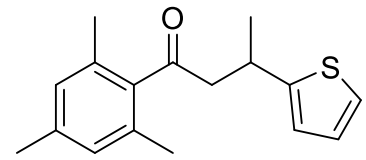

Chemical Formula: $\mathrm{C}_{17} \mathrm{H}_{20} \mathrm{OS}$

\section{- 1-mesityl-3-(thiophen-2-yl)butan-1-one (1j)}

According to general procedure, alkylation of 1-mesitylethan-1-one $(0.5 \mathrm{mmol})$ with 1 (thiophen-2-yl)ethan-1-ol (1.5 equiv, $96 \mathrm{mg}$ ) afforded the pure product $\mathbf{1 j}$ as a light yellow oil (99 $\mathrm{mg}, 73 \%$ ) by silica flash column chromatography (pentane/diethyl ether 98:2). ${ }^{1} \mathbf{H}-\mathbf{N M R}$ $\left(\mathrm{CDCl}_{3}, 500 \mathrm{MHz}\right): \delta 7.11(\mathrm{~d}, J=5.1 \mathrm{~Hz}, 1 \mathrm{H}), 6.90(\mathrm{t}, J=4.3 \mathrm{~Hz}, 1 \mathrm{H}), 6.87(\operatorname{app~d}, J=3.2 \mathrm{~Hz}$, $1 \mathrm{H}), 6.81(\mathrm{~s}, 2 \mathrm{H}), 3.83(\mathrm{sext}, J=6.8 \mathrm{~Hz}, 1 \mathrm{H}), 3.08\left(\mathrm{ABX}, J_{A X}=5.8 \mathrm{~Hz} ; J_{B A}=18.5 \mathrm{~Hz}, 1 \mathrm{H}\right), 2.94$ $\left(\mathrm{ABX}, J_{B X}=7.7 \mathrm{~Hz} ; J_{B A}=18.5 \mathrm{~Hz}, 1 \mathrm{H}\right), 2.27(\mathrm{~s}, 3 \mathrm{H}), 2.11(\mathrm{~s}, 6 \mathrm{H}), 1.44(\mathrm{~d}, J=6.9 \mathrm{~Hz}, 3 \mathrm{H}) \mathrm{ppm}$. ${ }^{13} \mathrm{C}-\mathrm{NMR}\left(\mathrm{CDCl}_{3}, 125 \mathrm{MHz}\right): \delta 208.4,150.5,139.2,138.4,132.6$ (2C), $128.5(2 \mathrm{C}), 126.5,123.1$, 122.8, 53.9, 30.1, 23.1 , 21.0, 18.9 (2C) ppm. IR (neat): v 2962, 2922, 1698, 1610, 1573, 1454, 
1376, 1344, 1152, 1033, 977, 849, 692, 597, $522 \mathrm{~cm}^{-1}$. HRMS (ESI-TOF) m/z [M + Na] : Calcd for $\mathrm{C}_{17} \mathrm{H}_{20} \mathrm{OSNa} 295.1133$; Found 295.1120.<smiles>Cc1cc(C)c(C(=O)CC(C)c2ccco2)c(C)c1</smiles>

Chemical Formula: $\mathrm{C}_{17} \mathrm{H}_{20} \mathrm{O}_{2}$

\section{- 3-(furan-2-yl)-1-mesitylbutan-1-one (1k)}

According to general procedure, alkylation of 1-mesitylethan-1-one $(0.5 \mathrm{mmol})$ with 1-(furan2-yl)ethan-1-ol (1.5 equiv, $96 \mathrm{mg}$ ) afforded the pure product $1 \mathrm{k}$ as a yellow oil (82 $\mathrm{mg}, 64 \%$ ) by silica flash column chromatography (pentane/diethyl ether 95:5). ${ }^{1} \mathbf{H}-\mathbf{N M R}\left(\mathbf{C D C l}_{\mathbf{3}}, \mathbf{5 0 0}\right.$ MHz): $\delta 7.30(\mathrm{~s}, 1 \mathrm{H}), 6.82(\mathrm{~s}, 2 \mathrm{H}), 6.27(\mathrm{app} \mathrm{t}, J=1.5 \mathrm{~Hz}, 1 \mathrm{H}), 6.03(\mathrm{~d}, J=3.0 \mathrm{~Hz}, 1 \mathrm{H}), 3.58$ (sext, $J=6.6 \mathrm{~Hz}, 1 \mathrm{H}), 3.13\left(\mathrm{ABX}, J_{A X}=5.4 \mathrm{~Hz} ; J_{A B}=18.6 \mathrm{~Hz}, 1 \mathrm{H}\right), 2.82\left(\mathrm{ABX}, J_{B X}=8.0 \mathrm{~Hz}\right.$; $\left.J_{B A}=18.6 \mathrm{~Hz}, 1 \mathrm{H}\right), 2.27(\mathrm{~s}, 3 \mathrm{H}), 2.15(\mathrm{~s}, 6 \mathrm{H}), 1.36(\mathrm{~d}, J=6.9 \mathrm{~Hz}, 3 \mathrm{H})$ ppm. ${ }^{13} \mathbf{C}-\mathbf{N M R}\left(\mathbf{C D C l}_{3}\right.$, $125 \mathrm{MHz}$ ): $\delta$ 208.5, 159.0, 140.8, 139.3, 138.3, 132.6 (2C), 128.5 (2C), 110.0, 104.0, 50.3, 28.2, 21.0, 19.2, 18.9 (2C) ppm. IR (neat): v 2969, 2923, 1698, 1611, 1507, 1453, 1403, 1278, 1151, 1009, 926, 850, 800, 729, $598 \mathrm{~cm}^{-1}$. HRMS (ESI-TOF) $\mathbf{m} / \mathbf{z}$ [M + H] : Calcd for $\mathrm{C}_{17} \mathrm{H}_{21} \mathrm{O}_{2} 257.1542 ;$ Found 257.1539.<smiles>Cc1cc(C)c(C(=O)CC2CCCc3ccccc32)c(C)c1</smiles>

Chemical Formula: $\mathrm{C}_{21} \mathrm{H}_{24} \mathrm{O}$

\section{- 1-mesityl-2-(1,2,3,4-tetrahydronaphthalen-1-yl)ethan-1-one (1I)}

According to general procedure, alkylation of 1-mesitylethan-1-one $(0.5 \mathrm{mmol})$ with $1,2,3,4$ tetrahydronaphthalen-1-ol (1.5 equiv, $111 \mathrm{mg}$ ) afforded the pure product $1 \mathrm{ll}$ as a colorless oil (106 mg, $73 \%$ ) by silica flash column chromatography (pentane/diethyl ether 95:5). ${ }^{1} \mathbf{H}-\mathbf{N M R}$ $\left(\mathrm{CDCl}_{3}, 500 \mathrm{MHz}\right): \delta$ 7.20-7.08 (m, 4H), $6.85(\mathrm{~s}, 2 \mathrm{H}), 3.69-3.66(\mathrm{~m}, 1 \mathrm{H}), 3.12-3.02(\mathrm{~m}, 2 \mathrm{H})$, 2.84-2.77 (m, 2H), $2.30(\mathrm{~s}, 3 \mathrm{H}), 2.24(\mathrm{~s}, 6 \mathrm{H}), 2.12-2.05(\mathrm{~m}, 1 \mathrm{H}), 1.86-1.80(\mathrm{~m}, 3 \mathrm{H}) \mathrm{ppm} .{ }^{13} \mathrm{C}-$ NMR (CDCl $\left.{ }_{3}, 125 \mathrm{MHz}\right): \delta 209.3,140.0,139.4,138.2,137.2,132.4(2 \mathrm{C}), 129.2,128.5(2 \mathrm{C})$, 128.4, 125.8, 125.8, 52.7, 32.3, 29.5, 28.4, 21.0, 19.6, 19.0 (2C) ppm. IR (neat): v 3014, 2923, 2862, 1698, 1611, 1489, 1450, 1348, 1284, 1151, 1035, 992, 850, 750, 738, 599, $531 \mathrm{~cm}^{-1}$. HRMS (ESI-TOF) m/z [M + Na] ${ }^{+}$: Calcd for $\mathrm{C}_{21} \mathrm{H}_{24} \mathrm{ONa} 315.1725$; Found 315.1725.

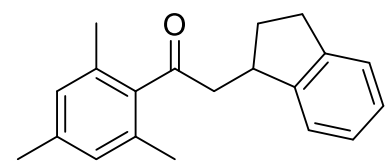

Chemical Formula: $\mathrm{C}_{20} \mathrm{H}_{22} \mathrm{O}$

\section{- 2-(2,3-dihydro-1H-inden-1-yl)-1-mesitylethan-1-one (1m)}

According to general procedure, alkylation of 1-mesitylethan-1-one $(0.5 \mathrm{mmol})$ with 2,3dihydro- $1 \mathrm{H}$-inden-1-ol (1.5 equiv, $101 \mathrm{mg}$ ) afforded the pure product $1 \mathrm{~m}$ as a yellow oil (109 $\mathrm{mg}, 78 \%)$ by silica flash column chromatography (pentane/diethyl ether 95:5). ${ }^{1} \mathbf{H}-\mathbf{N M R}$ $\left(\mathrm{CDCl}_{3}, 500 \mathrm{MHz}\right): \delta$ 7.24-7.20 (m, 2H), 7.17-7.15 (m, 2H), $6.84(\mathrm{~s}, 2 \mathrm{H}), 3.81-3.74(\mathrm{~m}, 1 \mathrm{H})$, $3.18(\mathrm{dd}, J=18.5 ; 4.6 \mathrm{~Hz}, 1 \mathrm{H}), 2.97-2.86(\mathrm{~m}, 3 \mathrm{H}), 2.53$ (app sext, $J=7.5 \mathrm{~Hz}, 1 \mathrm{H}$ ), 2.28 (s, $3 \mathrm{H}), 2.22(\mathrm{~s}, 6 \mathrm{H}), 1.71(\mathrm{td}, J=20.5,7.9 \mathrm{~Hz}, 1 \mathrm{H})$ ppm. ${ }^{13} \mathbf{C}-\mathbf{N M R}\left(\mathbf{C D C l}_{3}, \mathbf{1 2 5} \mathbf{M H z}\right): \delta$ 209.7, 
146.1, 143.9, 139.5, 138.4, 132.5 (2C), 128.6 (2C), 126.6, 126.2, 124.6, 123.5, 50.6, 39.8, 33.0, 31.4, 21.0, 19.2 (2C) ppm. IR (neat): v 2920, 1697, 1610, 1574, 1476, 1458, 1397, 1263, 1212, 1151, 1034, 988, 850, 747, 732, $590 \mathrm{~cm}^{-1}$. HRMS (ESI-TOF) $\mathbf{~ m / z ~ [ M ~ + ~ N a ] ~}{ }^{+}$Calcd for $\mathrm{C}_{20} \mathrm{H}_{22} \mathrm{ONa} 301.1568$; Found 301.1568.

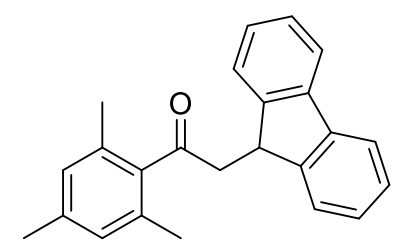

Chemical Formula: $\mathrm{C}_{24} \mathrm{H}_{22} \mathrm{O}$

\section{- 2-(9H-fluoren-9-yl)-1-(2,3,4,5,6-pentamethylphenyl)ethan-1-one (1n)}

According to general procedure, alkylation of 1 -mesitylethan-1-one $(0.5 \mathrm{mmol})$ with $9 \mathrm{H}$-fluoren9-ol (1.5 equiv, $137 \mathrm{mg}$ ) afforded the pure product $\mathbf{1 n}$ as a light yellow solid (129 $\mathrm{mg}, 79 \%$ ) by silica flash column chromatography (pentane/diethyl ether 95:5). ${ }^{1} \mathbf{H}-\mathbf{N M R}\left(\mathbf{C D C l}_{3}, \mathbf{5 0 0} \mathbf{~ M H z}\right)$ : $\delta 7.78(\mathrm{~d}, J=7.5 \mathrm{~Hz}, 2 \mathrm{H}), 7.63(\mathrm{~d}, J=7.5 \mathrm{~Hz}, 2 \mathrm{H}), 7.39(\mathrm{t}, J=7.5 \mathrm{~Hz}, 2 \mathrm{H}), 7.32(\mathrm{t}, J=7.5 \mathrm{~Hz}$, 2H), $6.85(\mathrm{~s}, 2 \mathrm{H}), 4.74(\mathrm{t}, J=6.4 \mathrm{~Hz}, 1 \mathrm{H}), 3.21(\mathrm{~d}, J=6.4 \mathrm{~Hz}, 2 \mathrm{H}), 2.29(\mathrm{~s}, 3 \mathrm{H}), 2.25(\mathrm{~s}, 6 \mathrm{H})$ ppm. ${ }^{13} \mathrm{C}-N M R\left(\mathrm{CDCl}_{3}, 125 \mathrm{MHz}\right): \delta 209.0,147.0,140.8(2 \mathrm{C}), 138.8(2 \mathrm{C}), 138.5,132.5(2 \mathrm{C})$, 128.5 (2C), 127.2 (2C), $127.1(2 \mathrm{C}), 124.8(2 \mathrm{C}), 119.8(2 \mathrm{C}), 49.5,41.9,21.0,19.2$ (2C) ppm. IR (neat): v 2988, 2901, 1698, 1609, 1449, 1393, 1250, 1066, 1056, 981, 850, 757, 736, 599, $427 \mathrm{~cm}^{-1}$. HRMS (ESI-TOF) $\mathbf{m} / \mathbf{z}$ [M + H] $]^{+}$: Calcd for $\mathrm{C}_{24} \mathrm{H}_{23} \mathrm{O} 327.1749$; Found 327.1756.<smiles>CCC(CC(=O)c1c(C)cc(C)cc1C)c1ccccc1</smiles>

Chemical Formula: $\mathrm{C}_{20} \mathrm{H}_{24} \mathrm{O}$

\section{- 1-mesityl-3-phenylpentan-1-one (10)}

According to general procedure, alkylation of 1-mesitylethan-1-one $(0.5 \mathrm{mmol})$ with 1 phenylpropan-1-ol (1.5 equiv, $103 \mu \mathrm{L}$ ) afforded the pure product 10 as a yellow oil $(76 \mathrm{mg}, 54$ $\%$ ) by silica flash column chromatography (pentane/diethyl ether 95:5). ${ }^{1} \mathbf{H}-\mathbf{N M R}\left(\mathbf{C D C l}_{3}, \mathbf{5 0 0}\right.$ MHz): $\delta 7.29-7.25(\mathrm{~m}, 2 \mathrm{H}), 7.21-7.17(\mathrm{~m}, 3 \mathrm{H}), 6.77(\mathrm{~s}, 2 \mathrm{H}), 3.28-3.22(\mathrm{~m}, 1 \mathrm{H}), 3.01(\mathrm{~d}, J=6.9$, $2 \mathrm{H}), 2.25(\mathrm{~s}, 3 \mathrm{H}), 2.00(\mathrm{~s}, 6 \mathrm{H}), 1.86-1.78(\mathrm{~m}, 1 \mathrm{H}), 1.65-1.58(\mathrm{~m}, 1 \mathrm{H}), 0.80(\mathrm{t}, J=7.3 \mathrm{~Hz}, 3 \mathrm{H})$ ppm. ${ }^{13} \mathrm{C}-\mathrm{NMR}\left(\mathrm{CDCl}_{3}, 125 \mathrm{MHz}\right): \delta$ 209.1, 144.5, 139.5, 138.2, 132.6 (2C), 128.5 (2C), 128.3 (2C), 127.9 (2C), 126.2, 51.8, 41.9, 29.3, 21.0, 18.9 (2C), 12.0 ppm. IR (neat): v 3028, 2960, 2924, 2873, 1698, 1610, 1493, 1463, 1401, 1342, 1267, 1152, 1031, 978, 945, 850, 757, 699, 599, $533 \mathrm{~cm}^{-1}$. HRMS (ESI-TOF) $\mathbf{m} / \mathbf{z}[\mathbf{M}+\mathrm{Na}]^{+}$: Calcd for $\mathrm{C}_{20} \mathrm{H}_{24} \mathrm{ONa}$ 303.1725; Found 303.1721 . 


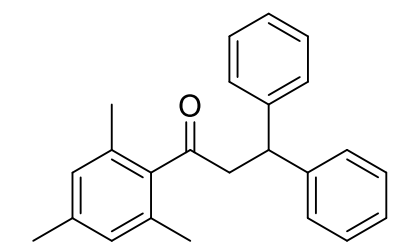

Chemical Formula: $\mathrm{C}_{24} \mathrm{H}_{24} \mathrm{O}$

\section{- 1-mesityl-3,3-diphenylpropan-1-one (1p)}

According to general procedure, alkylation of 1-mesitylethan-1-one $(0.5 \mathrm{mmol})$ with diphenylmethanol ( 3 equiv, $266 \mu \mathrm{L}$ ) afforded the pure product $1 \mathrm{p}$ as a colorless oil $(103 \mathrm{mg}$, $63 \%$ ) by silica flash column chromatography (pentane/diethyl ether 95:5). ${ }^{1} \mathbf{H}-\mathbf{N M R}\left(\mathbf{C D C l}_{3}\right.$, $500 \mathrm{MHz}$ ): $\delta 7.80$ (d, $J=7.9 \mathrm{~Hz}, 1 \mathrm{H}), 7.47$ (t, $J=7.5 \mathrm{~Hz}, 1 \mathrm{H}), 7.29-7.24(\mathrm{~m}, 6 \mathrm{H}), 7.15$ (t, $J=$ $6.8 \mathrm{~Hz}, 1 \mathrm{H}), 6.76(\mathrm{~s}, 2 \mathrm{H}), 4.79(\mathrm{t}, J=7.2 \mathrm{~Hz}, 1 \mathrm{H}), 3.48(\mathrm{~d}, J=7.2 \mathrm{~Hz}, 2 \mathrm{H}), 2.25(\mathrm{~s}, 3 \mathrm{H}), 1.89$ (s, 6H) ppm. ${ }^{13} \mathbf{C}-N M R\left(\mathbf{C D C l}_{3}, 125 \mathrm{MHz}\right): \delta 207.8,144.1,139.2,138.5,137.6,132.9,132.4$ (2C), 130.1, 128.6 (2C), 128.5 (2C), 128.3, 128.0 (2C), 126.4 (2C), 50.9, 45.4, 21.0, 18.8 (2C) ppm. IR (neat): $v$ 2934, 1698, 1608, 1563, 1476, 1463, 1397, 1254, 1232, 1136, 1024, 982 , 860, 744, $590 \mathrm{~cm}^{-1}$. HRMS (ESI-TOF) $\mathbf{m} / \mathbf{z}[\mathbf{M}+\mathbf{N a}]^{+}$: Calcd for $\mathrm{C}_{24} \mathrm{H}_{24} \mathrm{ONa} 351.1725$; Found 351.1721.

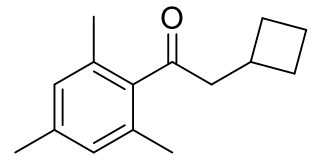

Chemical Formula: $\mathrm{C}_{15} \mathrm{H}_{20} \mathrm{O}$

\section{- 2-cyclobutyl-1-mesitylethan-1-one (1q)}

According to general procedure, alkylation of 1-mesitylethan-1-one $(0.5 \mathrm{mmol})$ with cyclopentanol (1.5 equiv, $59 \mu \mathrm{L}$ ) afforded the pure product $1 \mathrm{q}$ as a colorless oil (56 $\mathrm{mg}, 52 \%$ ) by silica flash column chromatography (pentane/diethyl ether 98:2). ${ }^{1} \mathbf{H}-\mathbf{N M R}\left(\mathbf{C D C l}_{\mathbf{3}}, \mathbf{5 0 0}\right.$ MHz): $\delta 6.83(\mathrm{~s}, 2 \mathrm{H}), 2.67(\mathrm{t}, J=7.4 \mathrm{~Hz}, 2 \mathrm{H}), 2.27(\mathrm{~s}, 3 \mathrm{H}), 2.19(\mathrm{~s}, 6 \mathrm{H}), 1.73-1.67(\mathrm{~m}, 2 \mathrm{H})$, 1.36-1.33 (m, 3H), 0.92-0.89 (m, 2H) ppm. ${ }^{13} \mathbf{C}-\mathbf{N M R}\left(\mathbf{C D C l}_{3}, \mathbf{1 2 5} \mathbf{~ M H z}\right): \delta 211.0,139.9,138.1$, $132.4(2 \mathrm{C}), 128.4(2 \mathrm{C}), 44.8,31.4,23.1,22.5,21.0,19.1$ (2C), 13.93 ppm. IR (neat): $v$ 2957, 2925, 1697, 1611, 1454, 1404, 1379, 1250, 1230, 1154, 1066, 849, 735, $599 \mathrm{~cm}^{-1}$. HRMS (ESI-TOF) $\mathbf{m} / \mathbf{z}[\mathbf{M}+\mathbf{H}]^{*}$ : Calcd for $\mathrm{C}_{16} \mathrm{H}_{23} \mathrm{O}$ 231.1749; Found 231.1743.<smiles>Cc1cc(C)c(C(=O)CC2CCCC2)c(C)c1</smiles>

Chemical Formula: $\mathrm{C}_{16} \mathrm{H}_{22} \mathrm{O}$

\section{- 2-cyclopentyl-1-mesitylethan-1-one (1r)}

According to general procedure, alkylation of 1-mesitylethan-1-one $(0.5 \mathrm{mmol})$ with cyclopentanol ( 1.5 equiv, $68 \mu \mathrm{L}$ ) afforded the pure product $1 \mathrm{r}$ as a light yellow oil $(98 \mathrm{mg}, 85$ $\%$ ) by silica flash column chromatography (pentane/diethyl ether 98:2). ${ }^{1} \mathbf{H}-\mathbf{N M R}\left(\mathbf{C D C l}_{3}, \mathbf{5 0 0}\right.$ MHz): $\delta 6.82(\mathrm{~s}, 2 \mathrm{H}), 2.73(\mathrm{~d}, J=6.9 \mathrm{~Hz}, 2 \mathrm{H}), 2.34$ (sept, $J=7.5 \mathrm{~Hz}, 1 \mathrm{H}$ ), $2.27(\mathrm{~s}, 3 \mathrm{H}), 2.19$ (s, 6H), 1.95-1.90 (m, 2H), 1.64-1.55 (m, 4H), 1.18-1.11 (m, 2H) ppm. ${ }^{13} \mathbf{C}-\mathbf{N M R}\left(\mathbf{C D C l}_{3}, \mathbf{1 2 5}\right.$ MHz): $\delta 210.6,139.9,138.1,132.5$ (2C), 128.5 (2C), 51.1, 34.9, 32.8 (2C), 25.0 (2C), 21.0, 19.1 (2C) ppm. IR (neat): v 2949, 2867, 1697, 1611, 1450, 1399, 1332, 1156, 1034, 983, 849, $600,527,427 \mathrm{~cm}^{-1}$. HRMS (ESI-TOF) $\mathbf{m} / \mathbf{z}[\mathbf{M}+\mathbf{H}]^{+}$: Calcd for $\mathrm{C}_{16} \mathrm{H}_{23} \mathrm{O}$ 231.1749; Found 231.1743. 
<smiles>Cc1cc(C)c(C(=O)CC2CCCCC2)c(C)c1</smiles>

Chemical Formula: $\mathrm{C}_{17} \mathrm{H}_{24} \mathrm{O}$

\section{- 2-cyclohexyl-1-mesitylethan-1-one (1s)}

According to general procedure, alkylation of 1-mesitylethan-1-one $(0.5 \mathrm{mmol})$ with cyclohexanol (1.5 equiv, $78 \mu \mathrm{L}$ ) afforded the pure product $1 \mathrm{~s}$ as a light yellow oil $(100 \mathrm{mg}, 82$ $\%$ ) by silica flash column chromatography (pentane/diethyl ether 98:2). ${ }^{1} \mathbf{H}-\mathbf{N M R}\left(\mathbf{C D C l}_{3}, \mathbf{5 0 0}\right.$ MHz): $\delta 6.82(\mathrm{~s}, 2 \mathrm{H}), 2.58(\mathrm{~d}, J=6.5 \mathrm{~Hz}, 2 \mathrm{H}), 2.27(\mathrm{~s}, 3 \mathrm{H}), 2.19(\mathrm{~s}, 6 \mathrm{H}), 2.07-2.00(\mathrm{~m}, 1 \mathrm{H})$, 1.84-1.81 (m, 2H), 1.72-1.67 (m, 3H), 1.38-1.30 (m, 2H), 1.20-1.13 (m, 1H), 1.02-0.95 (m, 2H) ppm. ${ }^{13} \mathrm{C}-\mathrm{NMR}\left(\mathrm{CDCl}_{3}, \mathbf{1 2 5} \mathrm{MHz}\right): \delta 210.0,139.9,138.1,132.34(2 \mathrm{C}), 128.5$ (2C), 52.4, 33.3 (2C), 32.6, 26.3, 26.1 (2C), 21.0, 19.0 (2C) ppm. IR (neat): v 2920, 2851, 1700, 1611, 1447, 1400, 1350, 1285, 1150, 1035, 995, 850, 760, 600, $533 \mathrm{~cm}^{-1}$. HRMS (ESI-TOF) m/z [M + H] : Calcd for $\mathrm{C}_{17} \mathrm{H}_{25} \mathrm{O} 245.1902$; Found 245.1905.<smiles>Cc1cc(C)c(C(=O)CC2CCCCCC2)c(C)c1</smiles>

Chemical Formula: $\mathrm{C}_{18} \mathrm{H}_{26} \mathrm{O}$

\section{- 2-cycloheptyl-1-mesitylethan-1-one (1t)}

According to general procedure, alkylation of 1-mesitylethan-1-one $(0.5 \mathrm{mmol})$ with cycloheptanol (1.5 equiv, $91 \mu \mathrm{L}$ ) afforded the pure product 1t as a yellow oil (102 $\mathrm{mg}, 79 \%)$ by silica flash column chromatography (pentane/diethyl ether 98:2). ${ }^{1} \mathbf{H}-\mathbf{N M R}\left(\mathrm{CDCl}_{3}, \mathbf{5 0 0}\right.$ MHz): $\delta 6.82(\mathrm{~s}, 2 \mathrm{H}), 2.63(\mathrm{~d}, J=6.6 \mathrm{~Hz}, 2 \mathrm{H}), 2.27(\mathrm{~s}, 3 \mathrm{H}), 2.23-2.19(\mathrm{~m}, 7 \mathrm{H}), 1.81-1.77(\mathrm{~m}$, $2 \mathrm{H}), 1.67-1.59(\mathrm{~m}, 4 \mathrm{H}), 1.53-1.48(\mathrm{~m}, 4 \mathrm{H}), 1.28-1.21$ (m, 2H) ppm. ${ }^{13} \mathbf{C}-\mathbf{N M R}\left(\mathrm{CDCl}_{3}, \mathbf{1 2 5} \mathbf{~ M H z}\right)$ : $\delta 210.2,139.9,138.1,132.5(2 \mathrm{C}), 128.5$ (2C), 53.2, 34.9 (2C), 34.4, 28.3 (2C), 26.3 (2C), 21.0, 19.1 (2C) ppm. IR (neat): v 2918, 2853, 1698, 1611, 1445, 1398, 1352, 1269, 1213, 1034, 983, 849, 600, $508 \mathrm{~cm}^{-1}$. HRMS (ESI-TOF) $\mathbf{m} / \mathbf{z}[\mathbf{M}+\mathbf{H}]^{+}:$Calcd for $\mathrm{C}_{18} \mathrm{H}_{27} \mathrm{O}$ 259.2062; Found 259.2055 .<smiles>Cc1cc(C)c(C(=O)CC2CCCCCCC2)c(C)c1</smiles>

Chemical Formula: $\mathrm{C}_{19} \mathrm{H}_{28} \mathrm{O}$

\section{- 2-cyclooctyl-1-mesitylethan-1-one (1u)}

According to general procedure, alkylation of 1-mesitylethan-1-one $(0.5 \mathrm{mmol})$ with cyclooctanol (1.5 equiv, $99 \mu \mathrm{L}$ ) afforded the pure product $1 \mathrm{u}$ as a light yellow oil $(103 \mathrm{mg}, 76$ $\%$ ) by silica flash column chromatography (pentane/diethyl ether 98:2). ${ }^{1} \mathbf{H}-\mathbf{N M R}\left(\mathbf{C D C l}_{3}, \mathbf{5 0 0}\right.$ MHz): $\delta 6.82(\mathrm{~s}, 2 \mathrm{H}), 2.63(\mathrm{~d}, J=6.5 \mathrm{~Hz}, 2 \mathrm{H}), 2.31-2.27(\mathrm{~m}, 4 \mathrm{H}), 2.19(\mathrm{~s}, 6 \mathrm{H}), 1.73-1.51(\mathrm{~m}$, $12 \mathrm{H}), 1.39-1.33$ (m, 2H) ppm. ${ }^{13} \mathbf{C}-\mathbf{N M R}\left(\mathbf{C D C l}_{3}, 125 \mathbf{~ M H z}\right): \delta 210.2,139.9,138.1,132.5(2 \mathrm{C})$, 128.5 (2C), 53.3, 32.5 (2C), 32.4, 27.1 (2C), 26.1, 25.2 (2C), 21.0, 19.1 (2C) ppm. IR (neat): $v$ 2916, 2853, 1698, 1611, 1446, 1398, 1352, 1278, 1152, 977, 849, $600 \mathrm{~cm}^{-1}$. HRMS (ESI-TOF) $\mathbf{m} / \mathbf{z}[\mathbf{M}+\mathbf{H}]^{+}$: Calcd for $\mathrm{C}_{19} \mathrm{H}_{29} \mathrm{O} 273.2218$; Found 273.2214 . 
<smiles>Cc1cc(C)c(C(=O)CC(C)C)c(C)c1</smiles>

Chemical Formula: $\mathrm{C}_{14} \mathrm{H}_{20} \mathrm{O}$

\section{- 1-mesityl-3-methylbutan-1-one ${ }^{2}$ (1v)}

In a $15 \mathrm{~mL}$ flame-dried Schlenk tube equipped with a stirring bar, 1-mesitylethan-1-one (0.5 $\mathrm{mmol}, 83 \mu \mathrm{L}$ ), $\mathrm{Me}_{3} \mathrm{NO}$ (2.2 mg, $2 \mathrm{~mol} \%$ ), iron complex Fe1 (4.56 mg, $2 \mathrm{~mol} \%$ ), $\mathrm{NaO}{ }^{\mathrm{t}} \mathrm{Bu}$ (48 $\mathrm{mg}, 1$ equiv) and isopropanol $(1.0 \mathrm{~mL})$ were poured in under an argon atmosphere. The mixture was rapidly stirred at room temperature for $2 \mathrm{~min}$ and then placed into a pre-heated oil bath at $110^{\circ} \mathrm{C}$ and stirred over 24 hours. The mixture was cooled-down to room temperature, filtrated over silica with diethyl ether and concentrated under reduced pressure. The conversion was determined by $1 \mathrm{H}-\mathrm{NMR}$ spectroscopy, and then, the residue was purified by flash chromatography on silica gel using pentane-diethyl ether (95:5) as eluent to afford the pure product $1 \mathrm{v}$ as a colorless oil (27 mg, 26\%). ${ }^{1} \mathrm{H}-\mathrm{NMR}\left(\mathrm{CDCl}_{3}, 500 \mathbf{M H z}\right): \delta 6.82(\mathrm{~s}, 2 \mathrm{H}), 2.59$ (d, $J=6.7 \mathrm{~Hz}, 2 \mathrm{H}$ ), 2.28 (app sept, $J=6.7 \mathrm{~Hz}, 1 \mathrm{H}$ ), 2.27 (s, 3H), 2.19 (s, 6H), 1.00 (d, $J=6.7$ $\mathrm{Hz}, 6 \mathrm{H})$ ppm. ${ }^{13} \mathrm{C}-\mathrm{NMR}\left(\mathrm{CDCl}_{3}, \mathbf{1 2 5} \mathbf{M H z}\right): \delta 210.2,139.8,138.1,132.5(2 \mathrm{C}), 128.5(2 \mathrm{C}), 53.7$, 23.6, 22.7 (2C), 21.0, 19.1 (2C) ppm.

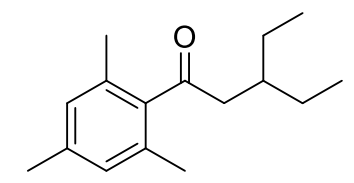

Chemical Formula: $\mathrm{C}_{16} \mathrm{H}_{24} \mathrm{O}$

\section{- 3-ethyl-1-mesitylpentan-1-one ${ }^{2}(1 w)$}

According to general procedure, alkylation of 1-mesitylethan-1-one $(0.5 \mathrm{mmol})$ with pentan-3ol $(1.5$ equiv, $81 \mu \mathrm{L})$ afforded the pure product $1 \mathrm{w}$ as a colorless oil $(61 \mathrm{mg}, 53 \%)$ by silica flash column chromatography (pentane/diethyl ether 95:5). ${ }^{1} \mathbf{H}-\mathbf{N M R}\left(\mathbf{C D C l}_{\mathbf{3}}, \mathbf{5 0 0} \mathbf{~ M H z}\right): \delta 6.83$ (s, 2H), 2.64 (d, J = 1.3 Hz, 2H), 2.27 (s, 3H), 2.20 (s, 6H), 1.95 (sept, $J=6.3 \mathrm{~Hz}, 1 \mathrm{H}$ ), 1.46$1.36(\mathrm{~m}, 4 \mathrm{H}), 0.87(\mathrm{t}, J=7.5 \mathrm{~Hz}, 6 \mathrm{H})$ ppm. ${ }^{13} \mathbf{C}-\mathbf{N M R}\left(\mathbf{C D C l}_{3}, \mathbf{1 2 5} \mathbf{~ M H z}\right): \delta 210.4,140.0,138.1$, 132.4 (2C), 128.5 (2C), 48.9, 35.4, 25.6 (2C), 21.0, $19.1(2 \mathrm{C}), 10.8(2 \mathrm{C}) \mathrm{ppm}$.

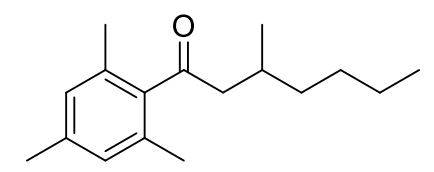

Chemical Formula: $\mathrm{C}_{17} \mathrm{H}_{26} \mathrm{O}$

\section{- 1-mesityl-3-methylheptan-1-one (1x)}

According to general procedure, alkylation of 1-mesitylethan-1-one $(0.5 \mathrm{mmol})$ with hexan-2ol (1.5 equiv, $95 \mu \mathrm{L}$ ) afforded the pure product $1 \mathrm{x}$ as a colorless oil ( $89 \mathrm{mg}, 72 \%)$ by silica flash column chromatography (pentane/diethyl ether 95:5). ${ }^{1} \mathbf{H}-\mathbf{N M R}\left(\mathbf{C D C l}_{3}, \mathbf{5 0 0} \mathbf{~ M H z}\right): \delta 6.82$ $(\mathrm{s}, 2 \mathrm{H}), 2.67\left(\mathrm{ABX}, J_{A X}=5.0 \mathrm{~Hz} ; J_{A B}=18.3 \mathrm{~Hz}, 1 \mathrm{H}\right), 2.51\left(\mathrm{ABX}, J_{B X}=8.0 \mathrm{~Hz} ; J_{B A}=18.3 \mathrm{~Hz}\right.$, $1 \mathrm{H}), 2.27(\mathrm{~s}, 3 \mathrm{H}), 2.20-2.13(\mathrm{~m}, 7 \mathrm{H}), 1.41-1.18(\mathrm{~m}, 6 \mathrm{H}), 1.00(\mathrm{~d}, J=6.6 \mathrm{~Hz}, 3 \mathrm{H}), 0.88(\mathrm{app} \mathrm{t}, J$ $=6.5 \mathrm{~Hz}, 3 \mathrm{H})$ ppm. ${ }^{13} \mathbf{C}-\mathrm{NMR}\left(\mathrm{CDCl}_{3}, 125 \mathrm{MHz}\right): \delta 210.3,139.9,138.1,132.4(2 \mathrm{C}), 128.5(2 \mathrm{C})$, 52.2, 36.6, 29.2, 28.1, 22.8, 21.0, 20.00, 19.1 (2C), 14.1 ppm. IR (neat): v 2957, 2923, 1698,

\footnotetext{
2 Thiyagarajan, S.; Gunanathan, C, J. Am. Chem. Soc, 2019, 141, 3822-3827.
} 
1611, 1455, 1402, 1378, 1250, 1066, 995, 850, 776, $600 \mathrm{~cm}^{-1}$. HRMS (ESI-TOF) m/z [M + H]+: Calcd for $\mathrm{C}_{17} \mathrm{H}_{27} \mathrm{O}$ 247.2062; Found 247.2059.

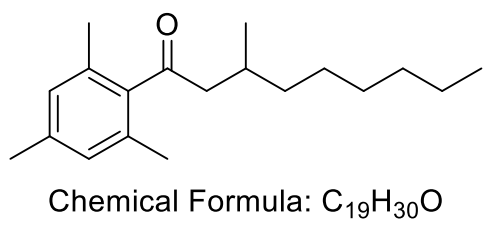

\section{- 1-mesityl-3-methylnonan-1-one (1y)}

According to general procedure, alkylation of 1-mesitylethan-1-one $(0.5 \mathrm{mmol})$ with octan-2-ol (1.5 equiv, $119 \mu \mathrm{L})$ afforded the pure product $1 \mathrm{y}$ as a yellow oil $(96 \mathrm{mg}, 70 \%)$ by silica flash column chromatography (pentane/diethyl ether 95:5). ${ }^{1} \mathbf{H}-\mathbf{N M R}\left(\mathbf{C D C l}_{3}, \mathbf{5 0 0} \mathbf{~ M H z}\right): \delta 6.82$ (s, 2H), $2.66\left(\mathrm{ABX}, J_{A X}=5.0 \mathrm{~Hz} ; J_{A B}=18.3 \mathrm{~Hz}, 1 \mathrm{H}\right), 2.50\left(\mathrm{ABX}, J_{B X}=8.0 \mathrm{~Hz} ; J_{B A}=18.3 \mathrm{~Hz}, 1 \mathrm{H}\right)$, $2.27(\mathrm{~s}, 3 \mathrm{H}), 2.20-2.14(\mathrm{~m}, 7 \mathrm{H}), 1.38-1.19(\mathrm{~m}, 10 \mathrm{H}), 0.99(\mathrm{~d}, J=6.7 \mathrm{~Hz}, 3 \mathrm{H}), 0.87$ (app t, $J=$ 6.6 Hz, 3H) ppm. ${ }^{13} \mathbf{C}-\mathrm{NMR}\left(\mathrm{CDCl}_{3}, 125 \mathrm{MHz}\right): \delta 210.2,139.9,138.1,132.5(2 \mathrm{C}), 128.5(2 \mathrm{C})$, 52.2, 36.9, 31.9, 29.4, 28.2, 26.9, 22.6, 21.0, 20.0, 19.1 (2C), 14.1 ppm. IR (neat): $v$ 2955, 2923, 2855, 1698, 1611, 1456, 1377, 1287, 1217, 1155, 955, 850, 724, $600 \mathrm{~cm}^{-1}$. HRMS (ESITOF) $\mathbf{m} / \mathbf{z}[\mathbf{M}+\mathbf{H}]^{+}$: Calcd for $\mathrm{C}_{19} \mathrm{H}_{31} \mathrm{O}$ 275.2375; Found 275.2375.

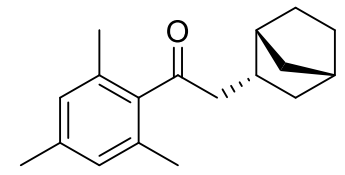

Chemical Formula: $\mathrm{C}_{18} \mathrm{H}_{24} \mathrm{O}$

\section{- 2-(bicyclo[2.2.1]heptan-2-yl)-1-mesitylethan-1-one (1z)}

According to general procedure, alkylation of 1-mesitylethan-1-one $(0.5 \mathrm{mmol})$ with exonorborneol (1.5 equiv, $84 \mathrm{mg}$ ) afforded the pure product $1 \mathrm{z}$ as a colorless oil (103 $\mathrm{mg}, 80 \%$ ) by silica flash column chromatography (pentane/diethyl ether 95:5). Product was obtained as one diastereoisomer (Structure confirmed with NOE analysis). ${ }^{1} \mathbf{H}-\mathbf{N M R}\left(\mathbf{C D C l}_{3}, \mathbf{5 0 0} \mathbf{~ M H z}\right): \delta$ $6.83(\mathrm{~s}, 2 \mathrm{H}), 2.76\left(\mathrm{ABX}, J_{A X}=6.0 \mathrm{~Hz} ; J_{B A}=17.8 \mathrm{~Hz}, 1 \mathrm{H}\right), 2.67\left(\mathrm{ABX}, J_{B X}=8.4 \mathrm{~Hz} ; J_{B A}=17.8\right.$ $\mathrm{Hz}, 1 \mathrm{H}$ ), 2.42-2.36 (m, 1H), 2.27 (s, 3H), 2.23 (br. s, 1H), 2.20 (br. s, 7H), 1.90 (tt, $J=3.6 ; 11.8$ $\mathrm{Hz}, 1 \mathrm{H})$ 1.56-1.25 (m, 5H), 1.10-1.07 (m, 1H), 0.68-0.64 (m, 1H) ppm. ${ }^{13} \mathrm{C}-\mathrm{NMR}\left(\mathbf{C D C l}_{3}, 125\right.$ MHz): $\delta 210.8,139.9,138.1,132.5$ (2C), 128.5 (2C), 48.4, 40.4, 39.8, 37.2, 37.1, 34.8, 30.1, 22.9, 21.0, 19.2 (2C) ppm. IR (neat): v 2947, 2867, 1696, 1611, 1451, 1399, 1271, 1152, 1035, 988, 850, 720, $600 \mathrm{~cm}^{-1}$. HRMS (ESI-TOF) $\mathbf{m} / \mathbf{z}[\mathbf{M}+\mathrm{H}]^{+}$: Calcd for $\mathrm{C}_{18} \mathrm{H}_{25} \mathrm{O}$ 257.1905; Found 257.1905

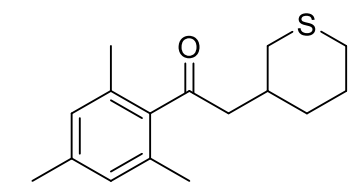

Chemical Formula: $\mathrm{C}_{16} \mathrm{H}_{22} \mathrm{OS}$

\section{- 1-mesityl-2-(tetrahydro-2H-thiopyran-3-yl)ethan-1-one (1aa)}

According to general procedure, alkylation of 1-mesitylethan-1-one $(0.5 \mathrm{mmol})$ with tetrahydro$2 \mathrm{H}$-thiopyran-3-ol (1.5 equiv, $89 \mathrm{mg}$ ) afforded the pure product 1 aa as a light brown solid (109 $\mathrm{mg}, 83 \%$ ) by silica flash column chromatography (pentane/diethyl ether 9:1). ${ }^{1} \mathbf{H}-\mathbf{N M R}\left(\mathbf{C D C l}_{3}\right.$, $500 \mathrm{MHz}): \delta 6.83(\mathrm{~s}, 2 \mathrm{H}), 2.76\left(\mathrm{ABX}, J_{A X}=5.6 ; J_{A B}=18.7 \mathrm{~Hz}, 1 \mathrm{H}\right), 2.77-2.72(\mathrm{~m}, 1 \mathrm{H}), 2.61$ $\left(\mathrm{ABX}, J_{B X}=4.1 \mathrm{~Hz} ; J_{B A}=18.7 \mathrm{~Hz}, 1 \mathrm{H}\right), 2.56-2.55(\mathrm{~m}, 2 \mathrm{H}), 2.48-2.44(\mathrm{~m}, 2 \mathrm{H}), 2.27(\mathrm{~s}, 3 \mathrm{H}), 2.18$ $(\mathrm{s}, 6 \mathrm{H}), 1.99-1.96(\mathrm{~m}, 1 \mathrm{H}), 1.91-1.88(\mathrm{~m}, 1 \mathrm{H}), 1.79-1.77(\mathrm{~m}, 1 \mathrm{H}), 1.26-1.20(\mathrm{~m}, 1 \mathrm{H}) \mathrm{ppm} .{ }^{13} \mathrm{C}-$ 
NMR (CDCl $3,125 \mathrm{MHz}): \delta 208.9,139.5,138.2,132.3$ (2C), 128.5 (2C), 50.6, 33.7, 32.5, 32.2, 28.6, 26.7, 21.0, 19.0 (2C) ppm. IR (neat): v 2971, 2917, 2901, 1691, 1610, 1450, 1425, 1380, 1251, 1066, 1057, 984, 925, 849, 725, 596, 515, $455 \mathrm{~cm}^{-1}$. HRMS (ESI-TOF) m/z [M + H]+: Calcd for $\mathrm{C}_{16} \mathrm{H}_{23} \mathrm{OS}$ 263.1470; Found 263.1462.

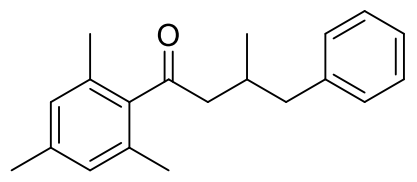

Chemical Formula: $\mathrm{C}_{20} \mathrm{H}_{24} \mathrm{O}$

\section{- 1-mesityl-3-methyl-4-phenylbutan-1-one (1ab)}

According to general procedure, alkylation of 1-mesitylethan-1-one $(0.5 \mathrm{mmol})$ with 1 phenylpropan-2-ol (1.5 equiv, $102 \mathrm{mg}$ ) afforded the pure product $1 \mathrm{ab}$ as a yellow oil $(78 \mathrm{mg}$, $56 \%$ ) by silica flash column chromatography (pentane/diethyl ether 95:5). ${ }^{1} \mathbf{H}-\mathbf{N M R}\left(\mathbf{C D C l}_{3}\right.$, $500 \mathrm{MHz}): \delta$ 7.27-7.23 (m, 2H), 7.18-7.15 (m, 3H), $6.78(\mathrm{~s}, 2 \mathrm{H}), 2.71-2.64(\mathrm{~m}, 2 \mathrm{H}), 2.55-2.46$ $(\mathrm{m}, 3 \mathrm{H}), 2.24(\mathrm{~s}, 3 \mathrm{H}), 2.09(\mathrm{~s}, 6 \mathrm{H}), 1.00(\mathrm{~d}, J=5.7 \mathrm{~Hz}, 3 \mathrm{H})$ ppm. ${ }^{13} \mathrm{C}-\mathrm{NMR}\left(\mathrm{CDCl}_{3}, \mathbf{1 2 5} \mathbf{~ M H z}\right)$ : $\delta 210.0,140.5,139.7,138.2,132.5(2 \mathrm{C}), 129.2(2 \mathrm{C}), 128.5(2 \mathrm{C}), 128.2(2 \mathrm{C}), 126.0,51.1,43.1$, 30.2, 21.0, 20.1, 19.0 (2C) ppm. IR (neat): v 3026, 2955, 2921, 1698, 1611, 1453, 1377, 1264, 1218, 1151, 1031, 995, 851, 742, 700, 603, $498 \mathrm{~cm}^{-1}$. HRMS (ESI-TOF) m/z [M + H] : Calcd for $\mathrm{C}_{20} \mathrm{H}_{25} \mathrm{O} 281.1905$; Found 281.1901.

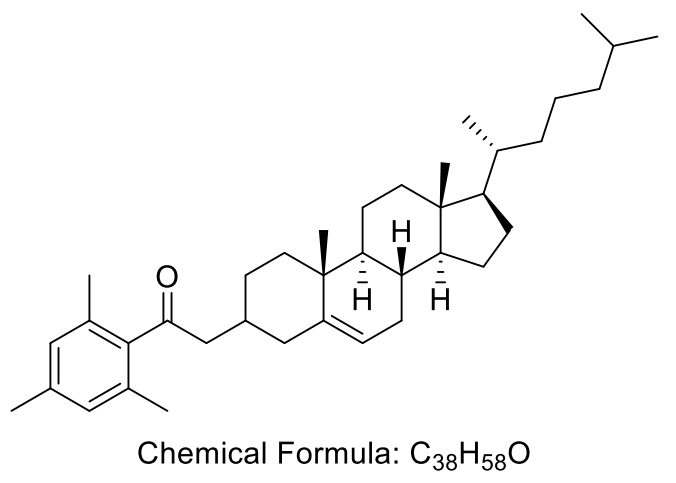

- 2-((8S,9S,10R,13R,14S,17R)-10,13-dimethyl-17-((R)-6-methylheptan-2-yl)$2,3,4,7,8,9,10,11,12,13,14,15,16,17$-tetradecahydro-1H-cyclopenta[a]phenanthren-3yl)-1-mesitylethan-1-one (1ac)

According to general procedure, alkylation of 1-mesitylethan-1-one $(0.5 \mathrm{mmol})$ with (3S,8S,9S, 10R, 13R,14S,17R)-10,13-dimethyl-17-((R)-6-methylheptan-2-yl)-

2,3,4,7,8,9,10,11,12,13,14,15,16,17-tetradecahydro- $1 \mathrm{H}$-cyclopenta[a]phenanthren-3-ol $\quad$ (1.5 equiv, $276 \mathrm{mg}$ ) afforded the pure product 1ac as a light colorless oil (101 mg, $38 \%$ ) by silica flash column chromatography (pentane/diethyl ether 95:5). ${ }^{1} \mathbf{H}-\mathbf{N M R}\left(\mathbf{C D C l}_{3}, \mathbf{5 0 0} \mathbf{~ M H z}\right): \delta 6.82$ $(\mathrm{s}, 2 \mathrm{H}), 5.16(\mathrm{~s}, 1 \mathrm{H}), 2.70-2.68(\mathrm{~m}, 1 \mathrm{H}), 2.63(\operatorname{app} \mathrm{d}, J=6.8 \mathrm{~Hz}, 2 \mathrm{H}), 2.27(\mathrm{~s}, 3 \mathrm{H}), 2.20(\mathrm{~s}, 6 \mathrm{H})$, 1.99-1.96 (m, 2H), 1.83-1.80 (m, 2H), 1.73-1.68 (m, 2H), 1.54-1.01 (m, 20H), 0.93-0.86 (m, $13 \mathrm{H}), 0.80-0.75(\mathrm{~m}, 1 \mathrm{H}), 0.68-0.64(\mathrm{~m}, 3 \mathrm{H}) \mathrm{ppm} .{ }^{13} \mathbf{C}-\mathbf{N M R}\left(\mathbf{C D C l}_{3}, \mathbf{1 2 5} \mathbf{~ M H z}\right): \delta 210.0,145.9$, 139.7, 138.2, 132.5 (2C), 128.5 (2C), 122.8, 56.2, 54.6, 51.7, 42.5, 40.0, 39.5, 37.6, 37.2, 36.2, 36.0, 35.8, 33.2, 32.7, 31.8, 28.2, 28.0, 26.6, 24.3, 23.8, 22.8, 22.6, 21.4, 21.0, 19.5, $19.2(2 \mathrm{C})$, 18.7, 12.0 (2C) ppm. IR (neat): v 2926, 2867, 1698, 1611, 1444, 1379, 1264, 1152, 1066, 1049, 979, 850, 737, 704, 677, $602 \mathrm{~cm}^{-1}$. HRMS (ESI-TOF) $\mathbf{m} / \mathbf{z}[\mathbf{M}+\mathbf{H}]^{+}:$Calcd for $\mathrm{C}_{38} \mathrm{H}_{59} \mathrm{O} 531.4566$; Found 531.4562. 


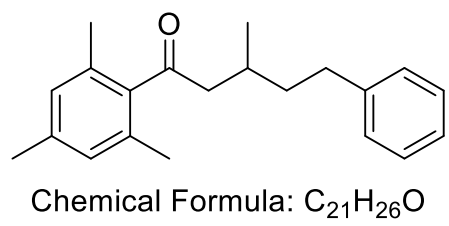

- 1-mesityl-3-methyl-5-phenylpentan-1-one (1ad)

According to general procedure, alkylation of 1-mesitylethan-1-one $(0.5 \mathrm{mmol})$ with 4 phenylbutan-2-ol (1.5 equiv, $113 \mathrm{mg}$ ) afforded the pure product $\mathbf{1 a d}$ as a yellow oil $(122 \mathrm{mg}$, $83 \%$ ) by silica flash column chromatography (pentane/diethyl ether $95: 5)$. ${ }^{1} \mathbf{H}-\mathbf{N M R}\left(\mathbf{C D C l}_{3}\right.$, $500 \mathrm{MHz})$ : $\delta 7.29-7.26(\mathrm{~m}, 2 \mathrm{H}), 7.19-7.17(\mathrm{~m}, 3 \mathrm{H}), 6.83(\mathrm{~s}, 2 \mathrm{H}), 2.71\left(\mathrm{ABX}, J_{A X}=5.1 \mathrm{~Hz} ; J_{A B}\right.$ $=18.4 \mathrm{~Hz}, 1 \mathrm{H}), 2.70-2.63(\mathrm{~m}, 2 \mathrm{H}), 2.57\left(\mathrm{ABX}, J_{B X}=7.9 \mathrm{~Hz} ; J_{B A}=18.4 \mathrm{~Hz}, 1 \mathrm{H}\right), 2.28-2.24(\mathrm{~m}$, $4 \mathrm{H}), 2.19(\mathrm{~s}, 6 \mathrm{H}), 1.78-1.70(\mathrm{~m}, 1 \mathrm{H}), 1.57-1.49(\mathrm{~m}, 1 \mathrm{H}), 1.08(\mathrm{~d}, J=6.7 \mathrm{~Hz}, 3 \mathrm{H}) \mathrm{ppm} .{ }^{13} \mathrm{C}-\mathrm{NMR}$ $\left(\mathrm{CDCl}_{3}, 125 \mathrm{MHz}\right): \delta$ 209.9, 142.4, 139.8, 138.2, 132.5 (2C), 128.5 (2C), 128.3 (2C), 128.3 (2C), 125.7, 52.1, 38.8, 33.5, 28.1, 21.0, 20.0, 19.1 (2C) ppm. IR (neat): v 3026, 2921, 1698, 1611, 1496, 1453, 1400, 1377, 1299, 1217, 1032, 994, 850, 742, 698, 600, $509 \mathrm{~cm}^{-1}$. HRMS (ESI-TOF) m/z [M + H]+: Calcd for $\mathrm{C}_{21} \mathrm{H}_{27} \mathrm{O}$ 295.2203; Found 295.2206.<smiles>Cc1c(C)c(CCO)c(C)c(C(=O)CC(C)c2ccc3c(c2)OCO3)c1C</smiles>

- 3-(benzo[d][1,3]dioxol-5-yl)-1-(2,3,4,5,6-pentamethylphenyl)butan-1-one (2b)

According to general procedure, alkylation of 1-(2,3,4,5,6-pentamethylphenyl)ethan-1-one (0.5 $\mathrm{mmol}, 95 \mathrm{mg}$ ) with 1-(benzo[d][1,3]dioxol-5-yl)ethan-1-ol (1.5 equiv, $125 \mathrm{mg}$ ) afforded the pure product $2 \mathrm{~b}$ as a white solid (127 $\mathrm{mg}, 68 \%)$ by silica flash column chromatography (pentane/diethyl ether 98:2). ${ }^{1} \mathbf{H}-\mathbf{N M R}\left(\mathbf{C D C l}_{3}, 500 \mathbf{~ M H z}\right): \delta 6.76-6.72(\mathrm{~m}, 3 \mathrm{H}), 5.92(\mathrm{~s}, 2 \mathrm{H})$, 3.46 (sext, $J=6.9 \mathrm{~Hz}, 1 \mathrm{H}), 2.96\left(\mathrm{ABX}, J_{A X}=5.9 \mathrm{~Hz} ; J_{A B}=18.9 \mathrm{~Hz}, 1 \mathrm{H}\right), 2.87\left(\mathrm{ABX}, J_{B X}=7.6\right.$ $\mathrm{Hz}$; $\left.J_{B A}=18.9 \mathrm{~Hz}, 1 \mathrm{H}\right), 2.23(\mathrm{~s}, 3 \mathrm{H}), 2.17(\mathrm{~s}, 6 \mathrm{H}), 2.02$ (br. s, 6H), 1.35 (d, J=6.9 Hz, 3H) ppm. ${ }^{13} \mathrm{C}-\mathrm{NMR}\left(\mathrm{CDCl}_{3}, \mathbf{1 2 5} \mathbf{~ M H z}\right): \delta$ 210.0, 147.5, 145.7, 140.7, 140.3, 135.3, 133.0, 127.3, 119.8, 108.1, 107.5, 100.7, 53.9, 33.9, 22.5, 16.9, 16.6, 15.9 (2C) ppm. IR (neat): v 2960, 2921, 1697, 1610, 1504, 1486, 1439, 1373, 1234, 1153, 1038, 987, 850, 808, 639, $601 \mathrm{~cm}^{-1}$. HRMS (ESITOF) $\mathbf{m} / \mathbf{z}[\mathbf{M}+\mathbf{H}]^{+}$: Calcd for $\mathrm{C}_{22} \mathrm{H}_{27} \mathrm{O}_{3} 339.1969$; Found 339.1971 .<smiles>Cc1c(C)c(C)c(C(=O)CC2CCc3ccccc32)c(C)c1C</smiles>

Chemical Formula: $\mathrm{C}_{22} \mathrm{H}_{26} \mathrm{O}$

- 2-(2,3-dihydro-1H-inden-1-yl)-1-(2,3,4,5,6-pentamethylphenyl)ethan-1-one ${ }^{1}$ (2c)

According to general procedure, alkylation of 1-(2,3,4,5,6-pentamethylphenyl)ethan-1-one (0.5 $\mathrm{mmol}, 95 \mathrm{mg}$ ) with 2,3-dihydro- $1 \mathrm{H}$-inden-1-ol (1.5 equiv, $101 \mathrm{mg}$ ) afforded the pure product 2c as a white solid (107 $\mathrm{mg}, 70 \%$ ) by silica flash column chromatography (pentane/diethyl ether 95:5). ${ }^{1} \mathrm{H}-\mathrm{NMR}\left(\mathrm{CDCl}_{3}, \mathbf{5 0 0} \mathbf{~ M H z}\right)$ : $\delta$ 7.34-7.30 (m, 2H), 7.27-7.13 (m, 2H), 3.91-3.85 (m, $\left.1 \mathrm{H}\right)$, $3.28(\mathrm{dd}, J=18.9 ; 4.5 \mathrm{~Hz}, 1 \mathrm{H}), 3.05-3.00(\mathrm{~m}, 2 \mathrm{H}), 2.93(\mathrm{dd}, J=15.8 ; 9.1 \mathrm{~Hz}, 1 \mathrm{H}), 2.75-2.68$ 
(m, 1H), 2.33 (s, 3H), $2.28(\mathrm{~s}, 6 \mathrm{H}), 2.23(\mathrm{~s}, 6 \mathrm{H}), 1.82$ (td, $J=20.5,7.9 \mathrm{~Hz}, 1 \mathrm{H})$ ppm. ${ }^{13} \mathrm{C}-\mathrm{NMR}$ $\left(\mathrm{CDCl}_{3}, 125 \mathrm{MHz}\right): \delta 210.9,146.1,143.9,140.4,135.4,133.1,127.3,126.6,126.2,124.5$, $123.5,51.3,39.6,33.1,31.4,17.1(2 \mathrm{C}), 16.7,15.9(2 \mathrm{C}) \mathrm{ppm}$.<smiles>Cc1c(C)c(C)c(C(=O)CC2c3ccccc3-c3ccccc32)c(C)c1C</smiles>

Chemical Formula: $\mathrm{C}_{26} \mathrm{H}_{26} \mathrm{O}$

- 2-(9H-fluoren-9-yl)-1-mesitylethan-1-one (2d)

According to general procedure, alkylation of 1-(2,3,4,5,6-pentamethylphenyl)ethan-1-one (0.5 mmol, $95 \mathrm{mg}$ ) with $9 H$-fluoren-9-ol (1.5 equiv, $137 \mathrm{mg}$ ) afforded the pure product $2 \mathbf{d}$ as a yellow solid (107 mg, $60 \%$ ) by silica flash column chromatography (pentane/diethyl ether 98:2). ${ }^{1} \mathrm{H}-\mathrm{NMR}\left(\mathrm{CDCl}_{3}, 500 \mathrm{MHz}\right): \delta 7.77$ (d, $\left.J=7.5 \mathrm{~Hz}, 2 \mathrm{H}\right), 7.65$ (d, $\left.J=7.5 \mathrm{~Hz}, 2 \mathrm{H}\right), 7.38$ (t, $J=7.5 \mathrm{~Hz}, 2 \mathrm{H}), 7.31(\mathrm{t}, J=7.5 \mathrm{~Hz}, 2 \mathrm{H}), 4.72(\mathrm{t}, J=6.4 \mathrm{~Hz}, 1 \mathrm{H}), 3.21(\mathrm{~d}, J=6.4 \mathrm{~Hz}, 2 \mathrm{H}), 2.24$ (s, 3H), 2.19 (s, 6H), 2.17 (s, 6H) ppm. ${ }^{13} \mathbf{C}-\mathbf{N M R}\left(\mathbf{C D C l}_{3}, \mathbf{1 2 5} \mathbf{~ M H z}\right): \delta$ 210.2, 147.1, 140.9, 139.7, 135.6, 133.1, 127.4, 127.2 (2C), 127.1 (2C), 124.8 (2C), 119.8 (2C), 50.3, 42.0, 17.2 (2C), 16.7, 15.9 (2C) ppm. IR (neat): v 2988, 2901, 1698, 1609, 1449, 1393, 1250, 1066, 1056, 981, 850, 757, 736, 599, $427 \mathrm{~cm}^{-1}$. HRMS (ESI-TOF) $\mathbf{m} / \mathbf{z}[\mathbf{M}+\mathbf{H}]^{+}$: Calcd for $\mathrm{C}_{26} \mathrm{H}_{27} \mathrm{O} 354.2017$; Found 354.2017.

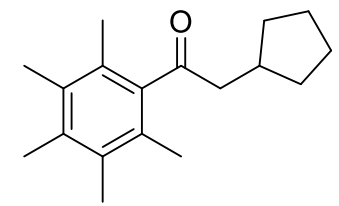

Chemical Formula: $\mathrm{C}_{18} \mathrm{H}_{26} \mathrm{O}$

\section{- 2-cyclopentyl-1-(2,3,4,5,6-pentamethylphenyl)ethan-1-one ${ }^{1}(2 e)$}

According to general procedure, alkylation of 1-(2,3,4,5,6-pentamethylphenyl)ethan-1-one (0.5 $\mathrm{mmol}, 95 \mathrm{mg}$ ) with cyclopentanol (1.5 equiv, $68 \mu \mathrm{L}$ ) afforded the pure product $2 \mathrm{e}$ as a white solid (101 mg, $78 \%$ ) by silica flash column chromatography (pentane/diethyl ether 98:2). ${ }^{1} \mathbf{H}$ NMR ( $\left.\mathrm{CDCl}_{3}, 500 \mathrm{MHz}\right): \delta 2.72(\mathrm{~d}, J=6.8 \mathrm{~Hz}, 2 \mathrm{H}), 2.36$ (sept, $\left.J=7.2 \mathrm{~Hz}, 1 \mathrm{H}\right), 2.23(\mathrm{~s}, 3 \mathrm{H}$ ), $2.18(\mathrm{~s}, 6 \mathrm{H}), 2.10(\mathrm{~s}, 6 \mathrm{H}), 1.98-1.92(\mathrm{~m}, 2 \mathrm{H}), 1.64-1.55(\mathrm{~m}, 4 \mathrm{H}), 1.17-1.13(\mathrm{~m}, 2 \mathrm{H}) \mathrm{ppm} .{ }^{13} \mathrm{C}-$ NMR (CDCl $3,125 \mathrm{MHz})$ : $\delta 211.8,140.8,135.2,133.0$ (2C), 127.3 (2C), 51.9, 34.6, 32.7 (2C), $25.0(2 \mathrm{C}), 17.1(2 \mathrm{C}), 16.7,15.9(2 \mathrm{C}) \mathrm{ppm}$.

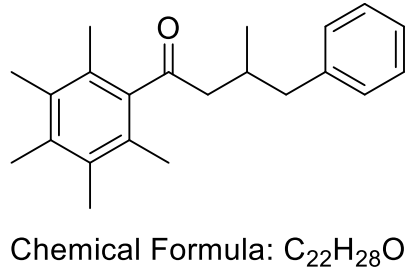

\section{- 3-methyl-1-(2,3,4,5,6-pentamethylphenyl)-4-phenylbutan-1-one (2f)}

According to general procedure, alkylation of 1-(2,3,4,5,6-pentamethylphenyl)ethan-1-one (0.5 $\mathrm{mmol}, 95 \mathrm{mg}$ ) with 1-phenylpropan-2-ol (1.5 equiv, $102 \mathrm{mg}$ ) afforded the pure product $\mathbf{2 f}$ as a yellow oil ( $83 \mathrm{mg}, 54 \%$ ) by silica flash column chromatography (pentane/diethyl ether 95:5). 
${ }^{1} \mathrm{H}-\mathrm{NMR}\left(\mathrm{CDCl}_{3}, \mathbf{5 0 0} \mathrm{MHz}\right): \delta$ 7.29-7.26 (m, 2H), 7.20-7.17 (m, 3H), 2.71-2.66 (m, 2H), 2.56$2.49(\mathrm{~m}, 3 \mathrm{H}), 2.22(\mathrm{~s}, 3 \mathrm{H}), 2.16(\mathrm{~s}, 6 \mathrm{H}), 2.02$ (br. s, 6H), $1.05(\mathrm{~d}, J=5.7 \mathrm{~Hz}, 3 \mathrm{H}) \mathrm{ppm} .{ }^{13} \mathrm{C}-$ NMR ( $\left.\mathrm{CDCl}_{3}, 125 \mathrm{MHz}\right): 211.1,140.6,140.6,135.3,133.0,129.2$ (2C), 128.2 (2C), 127.3, 125.9, 51.8, 43.1, 29.9, 20.1, 16.9 (2C), 16.7, 15.9 (2C) ppm. IR (neat): $v$ 3026, 2955, 2921, 1698, 1611, 1453, 1377, 1264, 1218, 1151, 1031, 995, 851, 742, 700, 603, $498 \mathrm{~cm}^{-1}$. HRMS (ESI-TOF) $\mathbf{m} / \mathbf{z}[\mathbf{M}+\mathbf{H}]^{+}$: Calcd for $\mathrm{C}_{22} \mathrm{H}_{29} \mathrm{O}$ 309.2156; Found 309.2160.

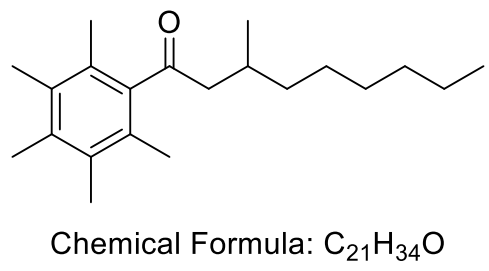

- 3-methyl-1-(2,3,4,5,6-pentamethylphenyl)nonan-1-one ${ }^{1}(2 \mathrm{~g})$

According to general procedure, alkylation of 1-(2,3,4,5,6-pentamethylphenyl)ethan-1-one (0.5 $\mathrm{mmol}, 95 \mathrm{mg}$ ) with octan-2-ol (1.5 equiv, $119 \mu \mathrm{L}$ ) afforded the pure product $\mathbf{2 g}$ as a colorless oil (95 mg, $63 \%$ ) by silica flash column chromatography (pentane/diethyl ether 95:5). ${ }^{\mathbf{1}} \mathbf{H}-\mathbf{N M R}$ $\left(\mathrm{CDCl}_{3}, 500 \mathrm{MHz}\right): \delta 2.67\left(\mathrm{ABX}, J_{A X}=5.0 \mathrm{~Hz} ; J_{A B}=18.3 \mathrm{~Hz}, 1 \mathrm{H}\right), 2.50\left(\mathrm{ABX}, J_{B X}=8.0 \mathrm{~Hz} ; J_{B A}\right.$ $=18.3 \mathrm{~Hz}, 1 \mathrm{H}), 2.27(\mathrm{~s}, 3 \mathrm{H}), 2.22-2.20(\mathrm{~m}, 7 \mathrm{H}), 2.13(\mathrm{~m}, 6 \mathrm{H}), 1.42-1.23(\mathrm{~m}, 10 \mathrm{H}), 1.04(\mathrm{~d}, J=$ $6.7 \mathrm{~Hz}, 3 \mathrm{H}), 0.89$ (app t, $J=7.0 \mathrm{~Hz}, 3 \mathrm{H})$ ppm. ${ }^{13} \mathrm{C}-\mathrm{NMR}\left(\mathbf{C D C l}_{3}, \mathbf{1 2 5} \mathbf{M H z}\right): \delta 211.3,140.8$, 135.2, 133.0 (2C), 127.2 (2C), 52.9, 36.8, 31.9, 29.4, 27.8, 26.9, 22.6, 20.0, 17.0 (2C), 16.6, $15.9(2 \mathrm{C}), 14.1 \mathrm{ppm}$.<smiles>Cc1c(C)c(C)c(C(=O)CC(C)CCc2ccccc2)c(C)c1C</smiles>

Chemical Formula: $\mathrm{C}_{23} \mathrm{H}_{30} \mathrm{O}$

- 3-methyl-1-(2,3,4,5,6-pentamethylphenyl)-5-phenylpentan-1-one ${ }^{3}(2 \mathrm{~h})$

According to general procedure, alkylation of 1-(2,3,4,5,6-pentamethylphenyl)ethan-1-one (0.5 $\mathrm{mmol}, 95 \mathrm{mg}$ ) with 4-phenylbutan-2-ol (1.5 equiv, $113 \mathrm{mg}$ ) afforded the pure product $2 \mathrm{~h}$ as a light yellow solid (118 mg, $73 \%$ ) by silica flash column chromatography (pentane/diethyl ether 95:5). ${ }^{1} \mathrm{H}-\mathrm{NMR}\left(\mathbf{C D C l}_{3}, \mathbf{5 0 0} \mathbf{~ M H z}\right): \delta$ 7.29-7.26 (m, 2H), 7.19-7.17 (m, 3H), $2.70\left(\mathrm{ABX}, \mathrm{J}_{A X}=\right.$ $\left.4.9 \mathrm{~Hz} ; J_{A B}=18.8 \mathrm{~Hz}, 1 \mathrm{H}\right), 2.64-2.60(\mathrm{~m}, 2 \mathrm{H}), 2.55\left(\mathrm{ABX}, J_{B X}=7.9 \mathrm{~Hz} ; J_{B A}=18.8 \mathrm{~Hz}, 1 \mathrm{H}\right)$, 2.28-2.22 (m, 4H), $2.18(\mathrm{~s}, 6 \mathrm{H}), 2.10(\mathrm{~s}, 6 \mathrm{H}), 1.79-1.72(\mathrm{~m}, 1 \mathrm{H}), 1.57-1.50(\mathrm{~m}, 1 \mathrm{H}), 1.10(\mathrm{~d}, J$ $=6.7 \mathrm{~Hz}, 3 \mathrm{H})$ ppm. ${ }^{13} \mathbf{C}-\mathrm{NMR}\left(\mathbf{C D C l}_{3}, 125 \mathrm{MHz}\right): \delta 211.0,142.5,140.7,135.3,133.1,128.3$ (4 C), $127.3,125.7,52.8,38.8,33.5,27.9,20.0,17.1$ (2C), 16.7, 15.9 (2C) ppm.

\footnotetext{
${ }^{3}$ Akhtar, W.; Cheong, C-B.; Frost, J-R.; Christensen, K-E.; Stevenson, N.G.; Donohoe,T.J. J. Am. Chem.
} Soc, 2017, 139, 2577-2580. 


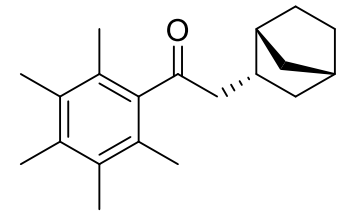

Chemical Formula: $\mathrm{C}_{20} \mathrm{H}_{28} \mathrm{O}$

\section{- 2-(bicyclo[2.2.1]heptan-2-yl)-1-(2,3,4,5,6-pentamethylphenyl)ethan-1-one (2i)}

According to general procedure, alkylation of 1-(2,3,4,5,6-pentamethylphenyl)ethan-1-one (0.5 $\mathrm{mmol}, 95 \mathrm{mg}$ ) with exo-norborneol (1.5 equiv, $84 \mathrm{mg}$ ) afforded the pure product $\mathbf{2} \mathbf{i}$ as a white solid (98 mg, $69 \%$ ) by silica flash column chromatography (pentane/diethyl ether 95:5). Product was obtained as one diastereoisomer. Structure was confirmed by single cristal X-ray crystallographic analysis after crystallization from $\mathrm{MeOH} .{ }^{1} \mathrm{H}-\mathrm{NMR}\left(\mathbf{C D C l}_{3}, \mathbf{5 0 0} \mathbf{~ M H z}\right): \delta 2.76$ $\left(A B X, J_{A X}=5.7 \mathrm{~Hz} ; J_{B A}=18.4 \mathrm{~Hz}, 1 \mathrm{H}\right), 2.64\left(\mathrm{ABX}, J_{B X}=8.4 \mathrm{~Hz} ; J_{B A}=18.4 \mathrm{~Hz}, 1 \mathrm{H}\right), 2.44-2.41$ $(\mathrm{m}, 1 \mathrm{H}), 2.25-2.23(\mathrm{~m}, 4 \mathrm{H}), 2.18(\mathrm{app} \mathrm{s}, 7 \mathrm{H}), 2.11(\mathrm{~s}, 6 \mathrm{H}), 2.0-1.94(\mathrm{~m}, 1 \mathrm{H})$ 1.54-1.28 $(\mathrm{m}, 5 \mathrm{H})$, 1.10-1.03 (m, $1 \mathrm{H}), 0.66(\mathrm{dd}, J=2.4 ; 5.3 \mathrm{~Hz}, 1 \mathrm{H})$ ppm. ${ }^{13} \mathbf{C}-\mathbf{N M R}\left(\mathrm{CDCl}_{3}, \mathbf{1 2 5} \mathbf{~ M H z}\right): \delta 212.1$, 140.9, 135.2, 133.5 (2C), 127.3, 49.3, 40.4, 39.8, 37.3, 37.1, 34.5, 30.1, 22.9, 17.2 (2C), 16.7, 15.9 (2C) ppm. IR (neat): v 2946, 2867, 1697, 1611, 1453, 1401, 1367, 1329, 1317, 1272 , 1169, 1110, 930, $682 \mathrm{~cm}^{-1}$. HRMS (ESI-TOF) m/z [M + H] : Calcd for $\mathrm{C}_{20} \mathrm{H}_{28} \mathrm{O} 285.1908$; Found 285.1906.

\section{Part 4: Functionalization of $\beta$-disubstituted carbonyl compounds}<smiles>CCCCOC(=O)CC(C)c1ccccc1</smiles>

Chemical Formula: $\mathrm{C}_{14} \mathrm{H}_{20} \mathrm{O}_{2}$

\section{- butyl 3-phenylbutanoate $(4 a)^{4}$}

To a stirred solution of ketone $1 \mathrm{a}(0.3 \mathrm{mmol}, 80 \mathrm{mg})$ in dichloromethane $(1.0 \mathrm{~mL})$ cooled at $17{ }^{\circ} \mathrm{C}$ was added dropwise bromine (2 equiv, $31 \mu \mathrm{L}$ ). The solution was stirred $15 \mathrm{~min}$, then $n$ butanol ( 3 equiv, $83 \mu \mathrm{L}$ ) was added. The reaction was then allowed to warm to room temperature and was stirred 20 hours. The reaction was then diluted in water and extracted three times with diethylether. The organic layer was then washed with sat. solution of $\mathrm{NaHCO}_{3}$, dried with $\mathrm{MgSO}_{4}$ and solvent was removed. The crude mixture was purified by flash chromatography on silica gel using pentane-ethyl acetate $(90: 10)$ as eluent to afford the desired product $\mathbf{4 a}$ as a colorless oil $(44 \mathrm{mg}, 66 \%){ }^{1} \mathrm{H}-\mathbf{N M R}\left(\mathbf{C D C l}_{3}, \mathbf{5 0 0} \mathbf{~ M H z}\right): \delta$ 7.31-7.28 $(\mathrm{m}, 2 \mathrm{H}), 7.23-7.18(\mathrm{~m}, 3 \mathrm{H}), 4.00(\mathrm{t}, J=6.7 \mathrm{~Hz}, 1 \mathrm{H}), 3.24$ (sext, $J=7.6 \mathrm{~Hz}, 1 \mathrm{H}), 2.60\left(\mathrm{ABX}, J_{A X}\right.$ $\left.=7.1 \mathrm{~Hz} ; J_{A B}=15.0 \mathrm{~Hz}, 1 \mathrm{H}\right), 2.52\left(\mathrm{ABX}, J_{B X}=8.1 \mathrm{~Hz} ; J_{B A}=15.0 \mathrm{~Hz}, 1 \mathrm{H}\right), 1.57-1.50(\mathrm{~m}, 2 \mathrm{H})$, 1.33-1.26 (m, 5H), 0.88 (t, $J=7.5 \mathrm{~Hz}, 3 \mathrm{H})$ ppm. ${ }^{13} \mathrm{C}-\mathrm{NMR}\left(\mathrm{CDCl}_{3}, \mathbf{1 2 5} \mathbf{M H z}\right): \delta 172.5,145.7$, $128.5(2 \mathrm{C}), 126.7$ (2C), 126.4, 64.2, 43.0, 36.5, 30.6, 21.9, 19.1, 13.7 ppm.

\footnotetext{
${ }^{4}$ Estevez, M.-C.; Galve, R.; Sanchez-Baeza, F.; Marco, M.-P. Chem. Eur. J, 2008, 14, 1906-1917.
} 
<smiles>CC(CC(=O)N1CCCC1)c1ccccc1</smiles>

\section{- 3-phenyl-1-(pyrrolidin-1-yl)butan-1-one ${ }^{1}$ (4b)}

To a stirred solution of ketone $1 \mathrm{a}(0.3 \mathrm{mmol}, 80 \mathrm{mg})$ in dichloromethane $(1.0 \mathrm{~mL})$ cooled at $17^{\circ} \mathrm{C}$ was added dropwise bromine (2 equiv, $31 \mu \mathrm{L}$ ). The solution was stirred $15 \mathrm{~min}$, then pyrrolidine ( 3 equiv, $74 \mu \mathrm{L}$ ) was added. The reaction was then allowed to warm to room temperature and was stirred 20 hours. The reaction was then diluted in water and extracted three times with diethylether. The organic layer was then washed with sat. solution of $\mathrm{NaHCO}_{3}$, dried with $\mathrm{MgSO}_{4}$ and solvent was removed. The crude mixture was purified by flash chromatography on silica gel using pentane-ethyl acetate $(1: 1)$ as eluent to afford the desired product $\mathbf{4 b}$ as a light yellow oil $(40 \mathrm{mg}, 61 \%){ }^{1} \mathbf{H}-\mathbf{N M R}\left(\mathbf{C D C l}_{3}, \mathbf{5 0 0} \mathbf{~ M H z}\right): \delta 7.30-7.25(\mathrm{~m}, 4 \mathrm{H})$, 7.21-7.17 (m, 1H), 3.46-3.36 (m, 3H), 3.34-3.30 (m, 1H), 3.14-3.10 (m, 1H), 2.51 (ABX, J $J_{A X}=$ $\left.6.6 \mathrm{~Hz} ; J_{A B}=14.7 \mathrm{~Hz}, 1 \mathrm{H}\right), 2.45\left(\mathrm{ABX}, J_{B X}=7.9 \mathrm{~Hz} ; J_{B A}=14.7 \mathrm{~Hz}, 1 \mathrm{H}\right), 1.88-1.74(\mathrm{~m}, 4 \mathrm{H})$, $1.34(\mathrm{~d}, J=7.0 \mathrm{~Hz}, 3 \mathrm{H})$ ppm. ${ }^{13} \mathrm{C}-\mathrm{NMR}\left(\mathrm{CDCl}_{3}, 125 \mathrm{MHz}\right): \delta 170.3,146.4,128.3$ (2C), 126.8 (2C), 126.2, 46.6, 45.5, 43.6, 36.3, 26.0, 24.3, 21.3 ppm.<smiles>CON(C)C(=O)CC(C)c1ccccc1</smiles>

Chemical Formula: $\mathrm{C}_{12} \mathrm{H}_{17} \mathrm{NO}_{2}$

\section{- N-methoxy-N-methyl-3-phenylbutanamide ${ }^{1}$ (4c)}

To a stirred solution of ketone $\mathbf{1 a}(0.3 \mathrm{mmol}, 80 \mathrm{mg})$ in dichloromethane $(1.0 \mathrm{~mL})$ cooled at $17^{\circ} \mathrm{C}$ was added dropwise bromine (2 equiv, $31 \mu \mathrm{L}$ ). The solution was stirred $15 \mathrm{~min}$, then pyrrolidine ( 3 equiv, $74 \mu \mathrm{L}$ ) was added. The reaction was then allowed to warm to room temperature and was stirred 20 hours. The reaction was then diluted in water and extracted three times with diethylether. The organic layer was then washed with sat. solution of $\mathrm{NaHCO}_{3}$, dried with $\mathrm{MgSO}_{4}$ and solvent was removed. The crude mixture was purified by flash chromatography on silica gel using pentane-ethyl acetate $(1: 1)$ as eluent to afford the desired product $4 \mathrm{c}$ as a light yellow oil (37 mg, $59 \%){ }^{1} \mathrm{H}-\mathrm{NMR}\left(\mathrm{CDCl}_{3}, \mathbf{5 0 0} \mathbf{M H z}\right): \delta$ 7.31-7.25 (m, 4H), 7.20-7.18 (m, 1H), $3.58(\mathrm{~s}, 3 \mathrm{H}), 3.34$ (sext, $J=7.6 \mathrm{~Hz}, 1 \mathrm{H}), 3.14(\mathrm{~s}, 3 \mathrm{H}), 2.74-2.70(\mathrm{~m}, 1 \mathrm{H})$, 2.67-2.59 (m, 1H), $1.31(\mathrm{~d}, J=7.0 \mathrm{~Hz}, 3 \mathrm{H})$ ppm. ${ }^{13} \mathbf{C}-\mathbf{N M R}\left(\mathbf{C D C l}_{3}, \mathbf{1 2 5} \mathbf{~ M H z}\right): \delta 146.6,128.5$ (2C), $127.0(2 \mathrm{C}), 126.8,126.3,61.2,40.3,35.8,29.7,21.7$ ppm. 


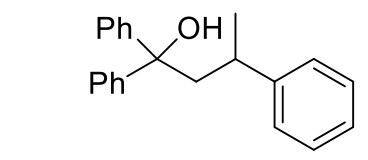

Chemical Formula: $\mathrm{C}_{22} \mathrm{H}_{22} \mathrm{O}$

\section{- 1,1,3-triphenylbutan-1-ol (4d)}

To a stirred solution of ketone $1 \mathrm{a}(0.3 \mathrm{mmol}, 80 \mathrm{mg})$ in dichloromethane $(1.0 \mathrm{~mL})$ cooled at $17^{\circ} \mathrm{C}$ was added dropwise bromine (2 equiv, $31 \mu \mathrm{L}$ ). The solution was stirred $15 \mathrm{~min}$, then phenylmagnesium bromide (4 equiv, $2.5 \mathrm{M}$ in THF) was added. The reaction was then allowed to warm to room temperature and was stirred 20 hours. The reaction was then quenched with $\mathrm{NH}_{4} \mathrm{Cl}$ and extracted three times with diethylether. The organic layer was then washed with sat. aq. solution of $\mathrm{NaHCO}_{3}$, dried with $\mathrm{MgSO}_{4}$ and solvent was removed. The crude mixture was purified by flash chromatography on silica gel using pentane-ethyl acetate (90:10) as eluent to afford the desired product $\mathbf{4 d}$ as a white solid $(53 \mathrm{mg}, 66 \%){ }^{1} \mathbf{H}-\mathbf{N M R}\left(\mathbf{C D C l}_{3}, \mathbf{5 0 0}\right.$ MHz): $\delta$ 7.46-7.44 (m, 2H), 7.41-7.35 (m, 4H), 7.32-7.26 (m, 5H), 7.23-7.19 (m, 2H), 7.13-7.11 (m, 2H), 2.81 (app sext, $J=6.9 \mathrm{~Hz}, 1 \mathrm{H}), 2.75-2.78(\mathrm{~m}, 2 \mathrm{H}), 1.94(\mathrm{~s}, 1 \mathrm{H}), 1.25(\mathrm{~d}, J=7.0 \mathrm{~Hz}$, 3H) ppm. ${ }^{13} \mathrm{C}-\mathrm{NMR}\left(\mathrm{CDCl}_{3}, \mathbf{1 2 5} \mathbf{~ M H z}\right): \delta 147.6,147.5,146.8,128.8$ (2C), 128.1 (2C), 128.0 (2C), 127.2 (2C), 126.7, 126.7, 126.4, 126.1 (2C), 125.8 (2C), 78.9, 49.9, 36.1, 24.5 ppm. HRMS (ESI-TOF) m/z [M + H] : Calcd for $\mathrm{C}_{22} \mathrm{H}_{23} \mathrm{O}$ 303.1753; Found 303.1756.<smiles>COc1ccc(C(=O)CC(C)c2ccccc2)cc1</smiles>

Chemical Formula: $\mathrm{C}_{17} \mathrm{H}_{18} \mathrm{O}_{2}$

\section{- 1-(4-methoxyphenyl)-3-phenylbutan-1-one ${ }^{5}(4 \mathrm{e})$}

In a $5 \mathrm{~mL}$ flame-dried Schlenk equipped with a stirring bar, ketone $1 \mathrm{a}(0.3 \mathrm{mmol}, 80 \mathrm{mg})$, anisole (2 equiv, $98 \mu \mathrm{L}$ ), and $\mathrm{TfOH}$ (1.2 equiv, $32 \mu \mathrm{L}$ ) were poured in. The solution was then stirred $90 \mathrm{~min}$ at $100^{\circ} \mathrm{C}$. The reaction was then cooled to room temperature and diluted in water $(20 \mathrm{~mL})$. The mixture was then extracted with $\mathrm{CH}_{2} \mathrm{Cl}_{2}(2 \times 20 \mathrm{~mL})$ and the organic phase was washed with sat. aq. $\mathrm{NaHCO}_{3}(20 \mathrm{~mL})$, dried over $\mathrm{MgSO}_{4}$ and concentrated in vacuo. Pure product $\mathbf{4 e}$ was afforded by silica flash column chromatography (pentane/diethyl ether 95:5) as a colorless oil (42 mg, $55 \%)$. ${ }^{1} \mathrm{H}-\mathrm{NMR}\left(\mathrm{CDCl}_{3}, \mathbf{5 0 0} \mathbf{M H z}\right): \delta 7.91(\mathrm{~d}, J=8.9 \mathrm{~Hz}, 2 \mathrm{H})$, 7.32-7.26 (m, 4H), 7.21-7.18 (m, 1H), $6.90(\mathrm{~d}, J=8.9 \mathrm{~Hz}, 2 \mathrm{H}), 3.86(\mathrm{~s}, 3 \mathrm{H}), 3.46$ (sext, $J=7.2$ $\mathrm{Hz}, 1 \mathrm{H}$ ), $3.22\left(\mathrm{ABX}, J_{A X}=5.7 \mathrm{~Hz} ; J_{A B}=16.2 \mathrm{~Hz}, 1 \mathrm{H}\right), 3.11\left(\mathrm{ABX}, J_{B X}=8.4 \mathrm{~Hz} ; J_{B A}=16.2 \mathrm{~Hz}\right.$, $1 \mathrm{H}), 1.32(\mathrm{~d}, J=7.0 \mathrm{~Hz}, 3 \mathrm{H}) \mathrm{ppm} .{ }^{13} \mathrm{C}-\mathrm{NMR}\left(\mathbf{C D C l}_{3}, 125 \mathbf{M H z}\right): \delta 197.7,163.4,146.7,130.4$ (2C), 130.3, 128.5 (2C), 126.9 (2C), 126.2, 113.7 (2C), 55.5, 46.7, 35.7, 21.8 ppm.

\footnotetext{
${ }^{5}$ Kalutharage, N.; Yi. S, C, Angew. Chem. Int. Ed, 2013, 52, 13651-13655.
} 
Scheme S1: Deuterium-labelling experiment<smiles>CC(=O)c1c(C)cc(C)cc1C</smiles><smiles>Cc1cc(C)c(C(=O)CC(C)(C)c2ccccc2)c(C)c1</smiles>

Chemical Formula: $\mathrm{C}_{19} \mathrm{H}_{21} \mathrm{DO}$

\author{
$\mathrm{Fe} 1(2 \mathrm{~mol} \%)$ \\ $\underset{\mathrm{NaO}{ }^{\mathrm{B}} \mathrm{Bu}(1 \text { equiv), Toluene }}{\stackrel{\mathrm{Me}_{3} \mathrm{NO}(4 \mathrm{~mol} \%)}{\longrightarrow}}$ \\ $110^{\circ} \mathrm{C}, 24 \mathrm{~h}$
}

\section{- 1-mesityl-3-phenylbutan-1-one- $d_{1}\left(1 \mathrm{a}-d_{1}\right)$}

In a $15 \mathrm{~mL}$ flame-dried Schlenk tube equipped with a stirring bar, 1-mesitylethan-1-one (0.5 mmol, $83 \mu \mathrm{L}$ ), 1-phenylethan-1-ol- $d_{1}(1.5$ equiv, $92 \mathrm{mg}), \mathrm{Me}_{3} \mathrm{NO}(2.2 \mathrm{mg}, 2 \mathrm{~mol} \%)$, iron complex Fe1 (4.56 mg, 2 mol \%), NaO'Bu (48 mg, 1 equiv) and toluene (1.0 mL) were poured in under an argon atmosphere. The mixture was rapidly stirred at room temperature for 2 min and then placed into a pre-heated oil bath at $110^{\circ} \mathrm{C}$ and stirred over 24 hours. The mixture was cooled-down to room temperature, filtrated over silica with diethyl ether and concentrated under reduced pressure. The conversion was determined by ${ }^{1} \mathrm{H}-\mathrm{NMR}$ spectroscopy, and then, the residue was purified by flash chromatography on silica gel using pentane-diethyl ether (98:2) as eluent to afford the desired product $\mathbf{1 a}-\boldsymbol{d}_{1}(82 \%)$. ${ }^{1} \mathbf{H}-\mathbf{N M R}\left(\mathbf{C D C l}_{3}, \mathbf{5 0 0} \mathbf{~ M H z}\right): \delta 7.27-$ $7.21(\mathrm{~m}, 4 \mathrm{H}), 7.17-7.14(\mathrm{~m}, 1 \mathrm{H}), 6.76(\mathrm{~s}, 2 \mathrm{H}), 2.98\left(\mathrm{AB}, J_{A B}=18.4 \mathrm{~Hz}, 1 \mathrm{H}\right), 2.91\left(\mathrm{AB}, J_{B A}=\right.$ $18.4 \mathrm{~Hz}, 1 \mathrm{H}), 2.23(\mathrm{~s}, 3 \mathrm{H}), 2.03(\mathrm{~s}, 6 \mathrm{H}), 1.33(\mathrm{~s}, 3 \mathrm{H}) \mathrm{ppm}$. 


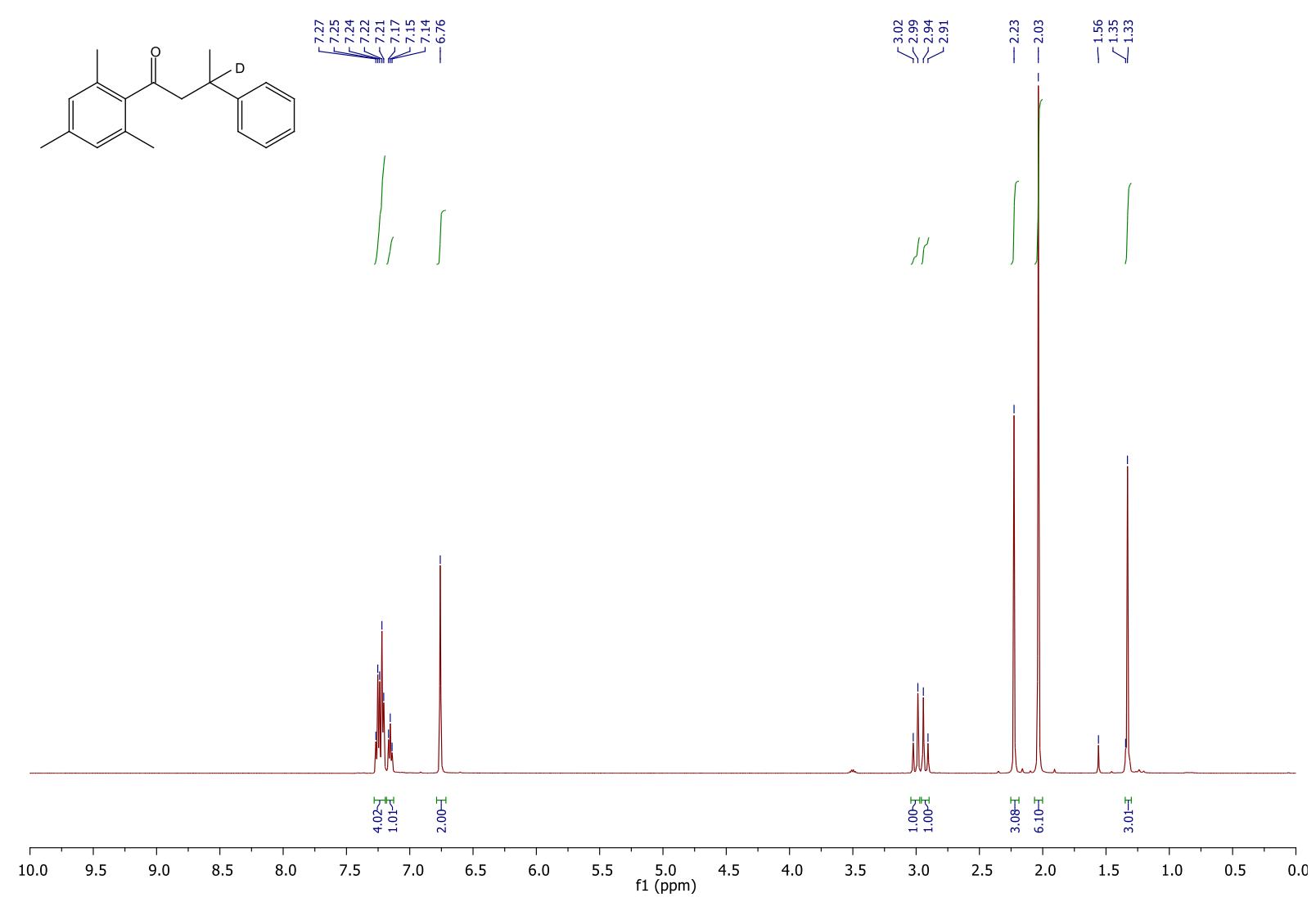

Figure S1: ${ }^{1} \mathrm{H}-\mathrm{NMR}(500 \mathrm{MHz})$ in $\mathrm{CDCl}_{3}$ of $1 \mathrm{a}-\mathrm{d}_{1}$ 
Part 6: NMR spectra of products

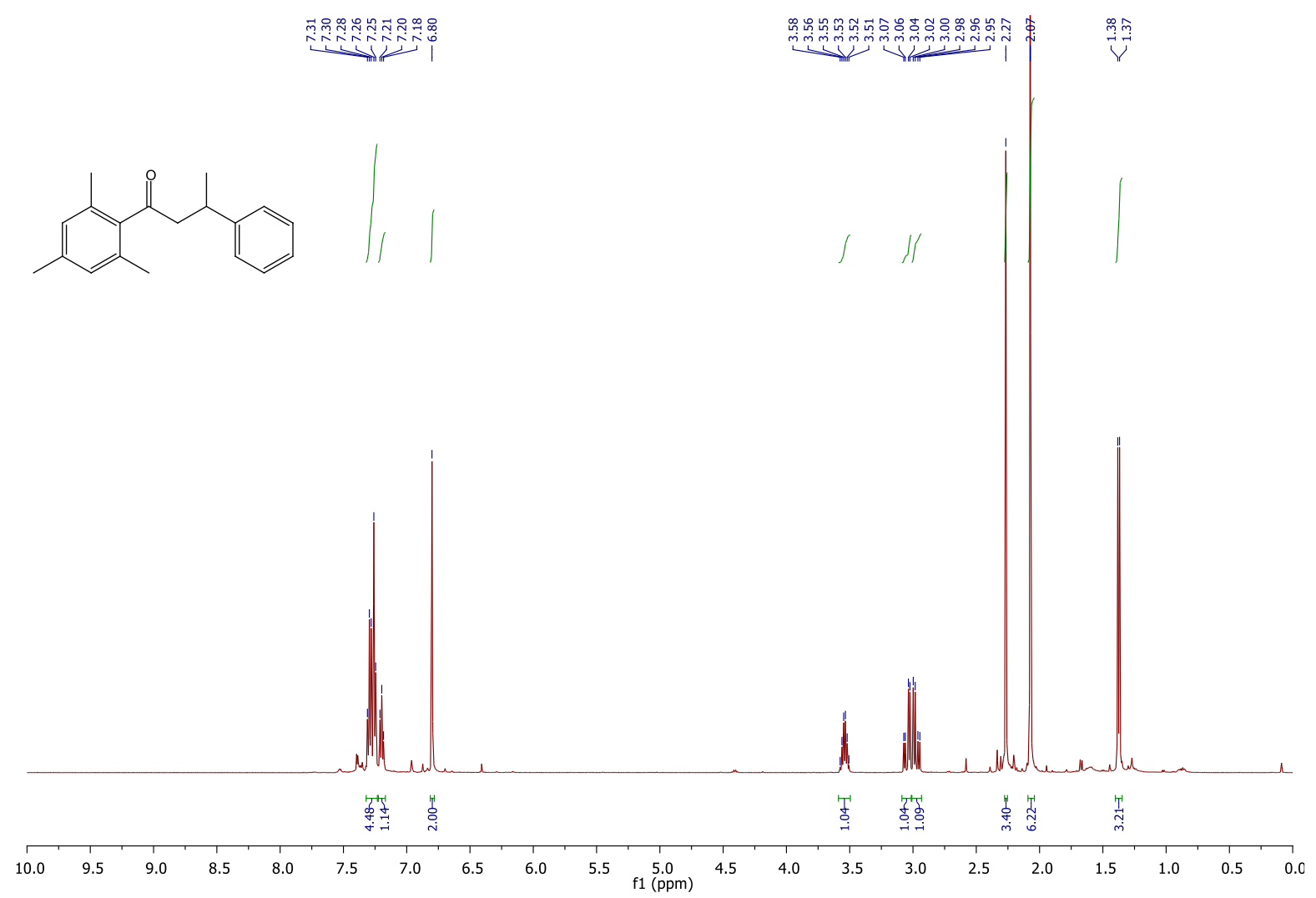

Figure S2: ${ }^{1} \mathrm{H}-\mathrm{NMR}(500 \mathrm{MHz})$ in $\mathrm{CDCl}_{3}$ of $1 \mathrm{a}$
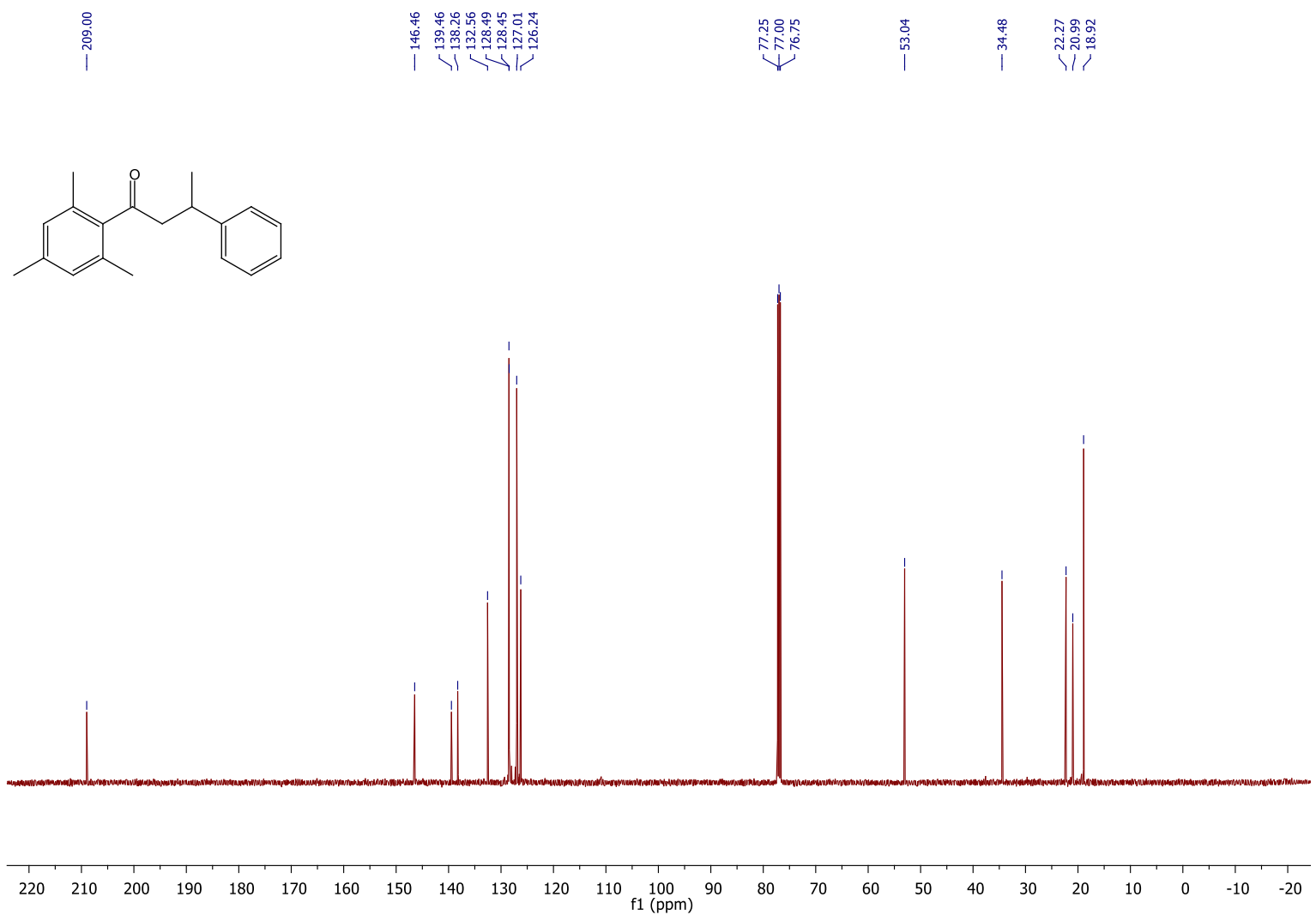

Figure S3: ${ }^{13} \mathrm{C}-\mathrm{NMR}(125 \mathrm{MHz})$ in $\mathrm{CDCl}_{3}$ of $1 \mathrm{a}$ 


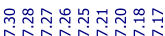

꾹
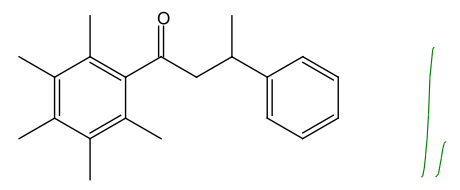

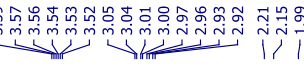

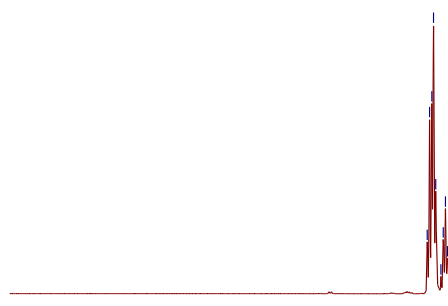

Figure S4: ${ }^{1} \mathrm{H}-\mathrm{NMR}(500 \mathrm{MHz})$ in $\mathrm{CDCl}_{3}$ of $2 \mathrm{a}$
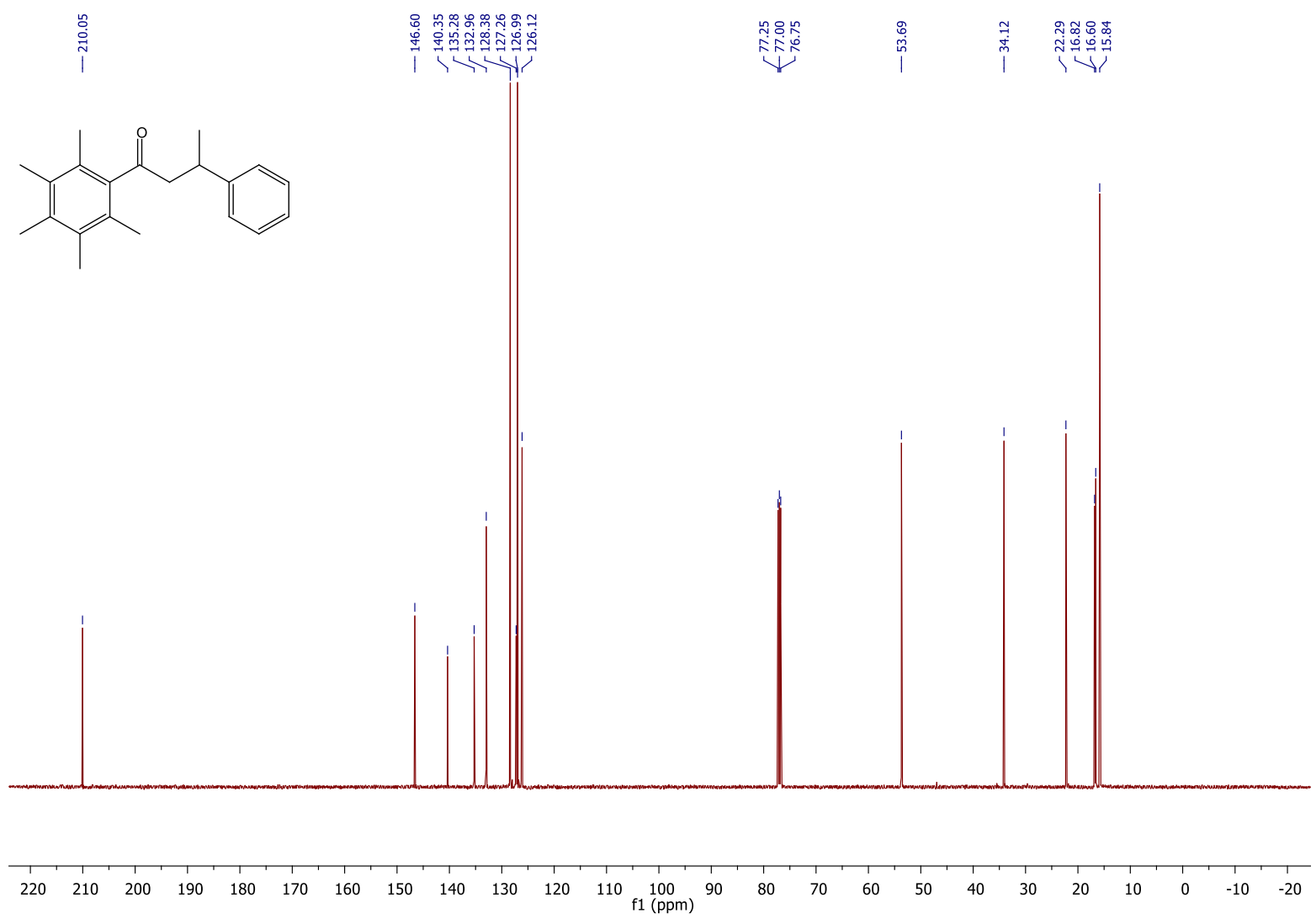

Figure S5: ${ }^{13} \mathrm{C}$-NMR (125 MHz) in $\mathrm{CDCl}_{3}$ of $2 \mathrm{a}$

S-25 


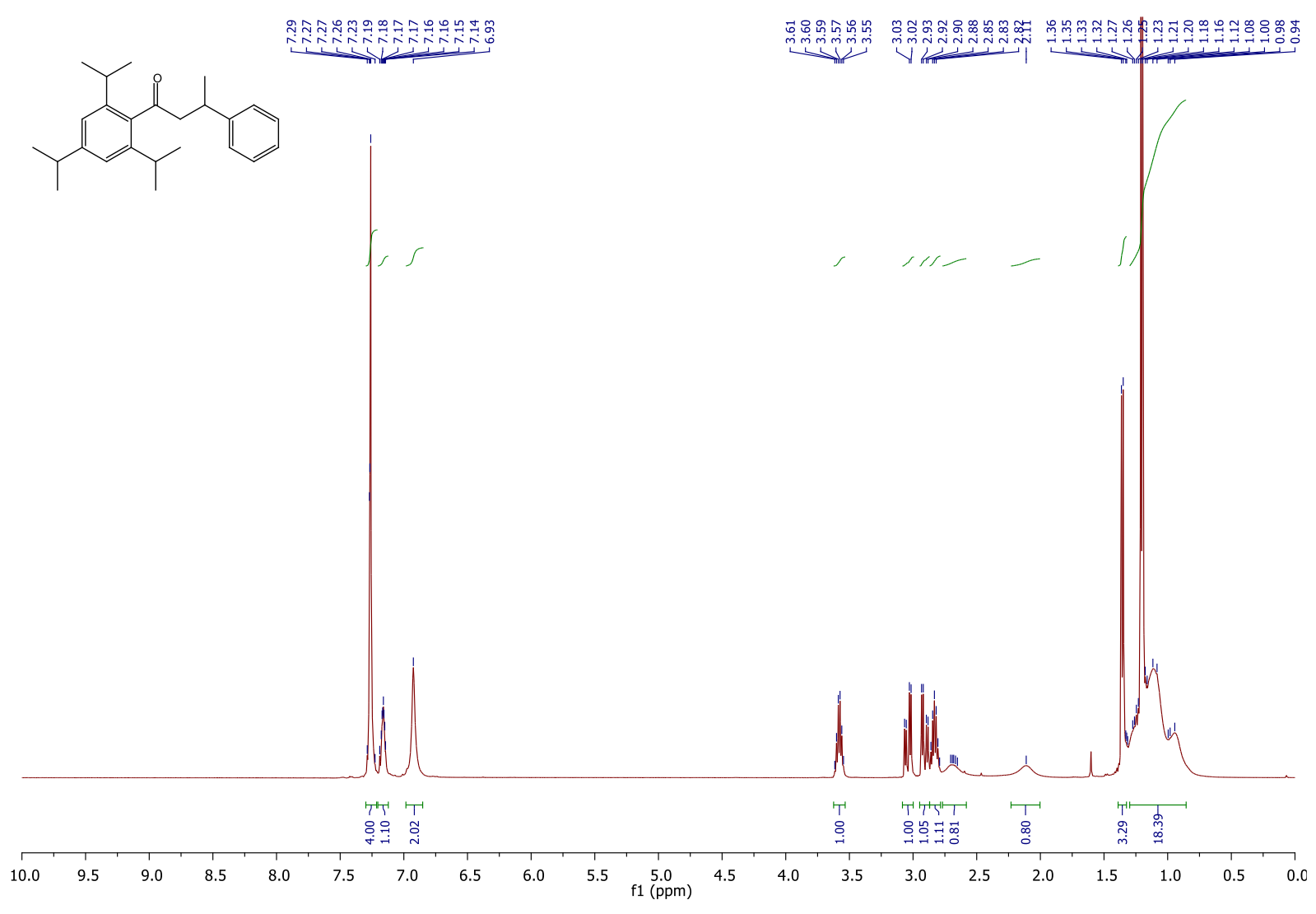

Figure S6: ${ }^{1} \mathrm{H}-\mathrm{NMR}(500 \mathrm{MHz})$ in $\mathrm{CDCl}_{3}$ of $3 \mathrm{a}$
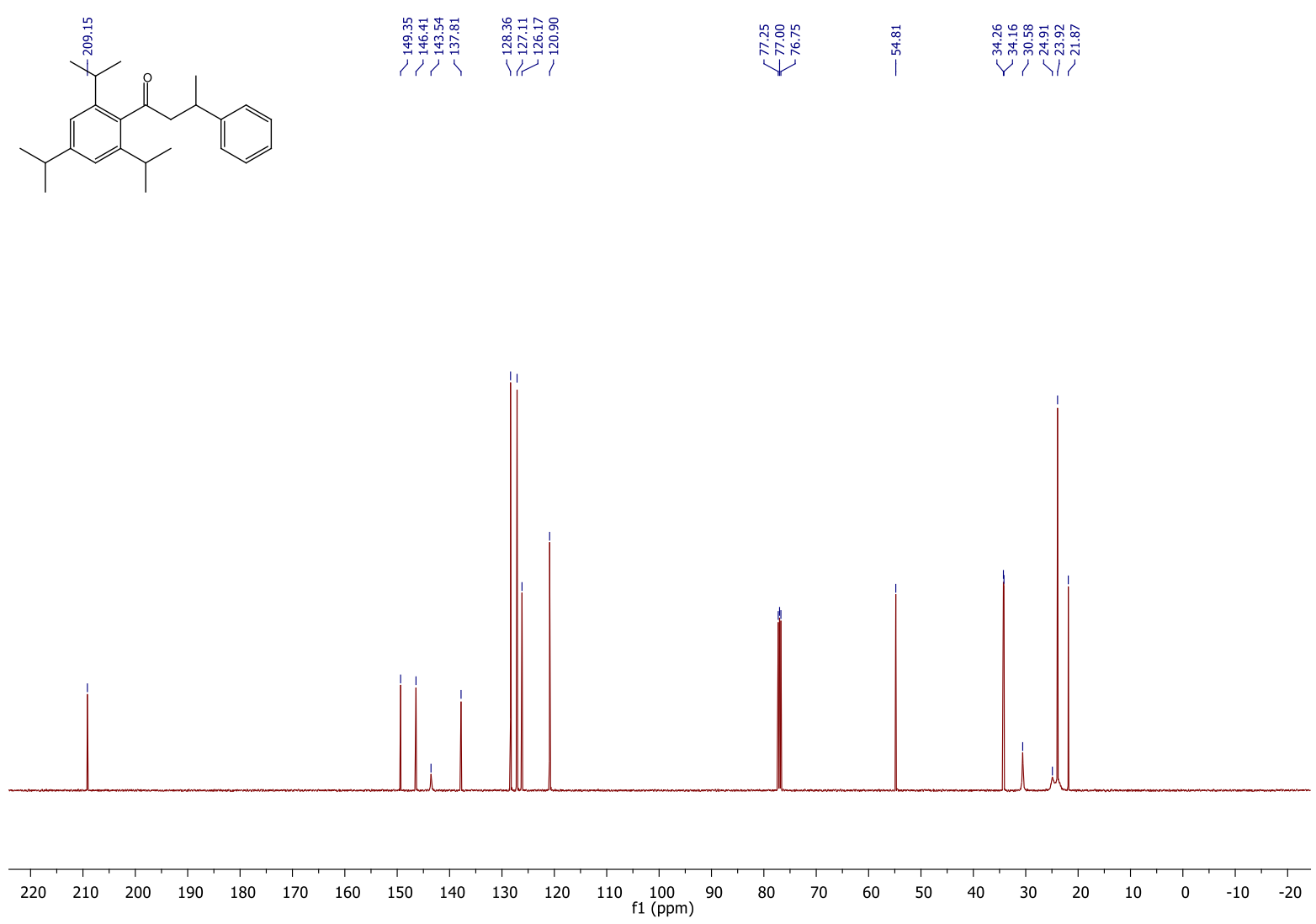

Figure S7: ${ }^{13} \mathrm{C}-\mathrm{NMR}(125 \mathrm{MHz})$ in $\mathrm{CDCl}_{3}$ of $3 a$

S-26 


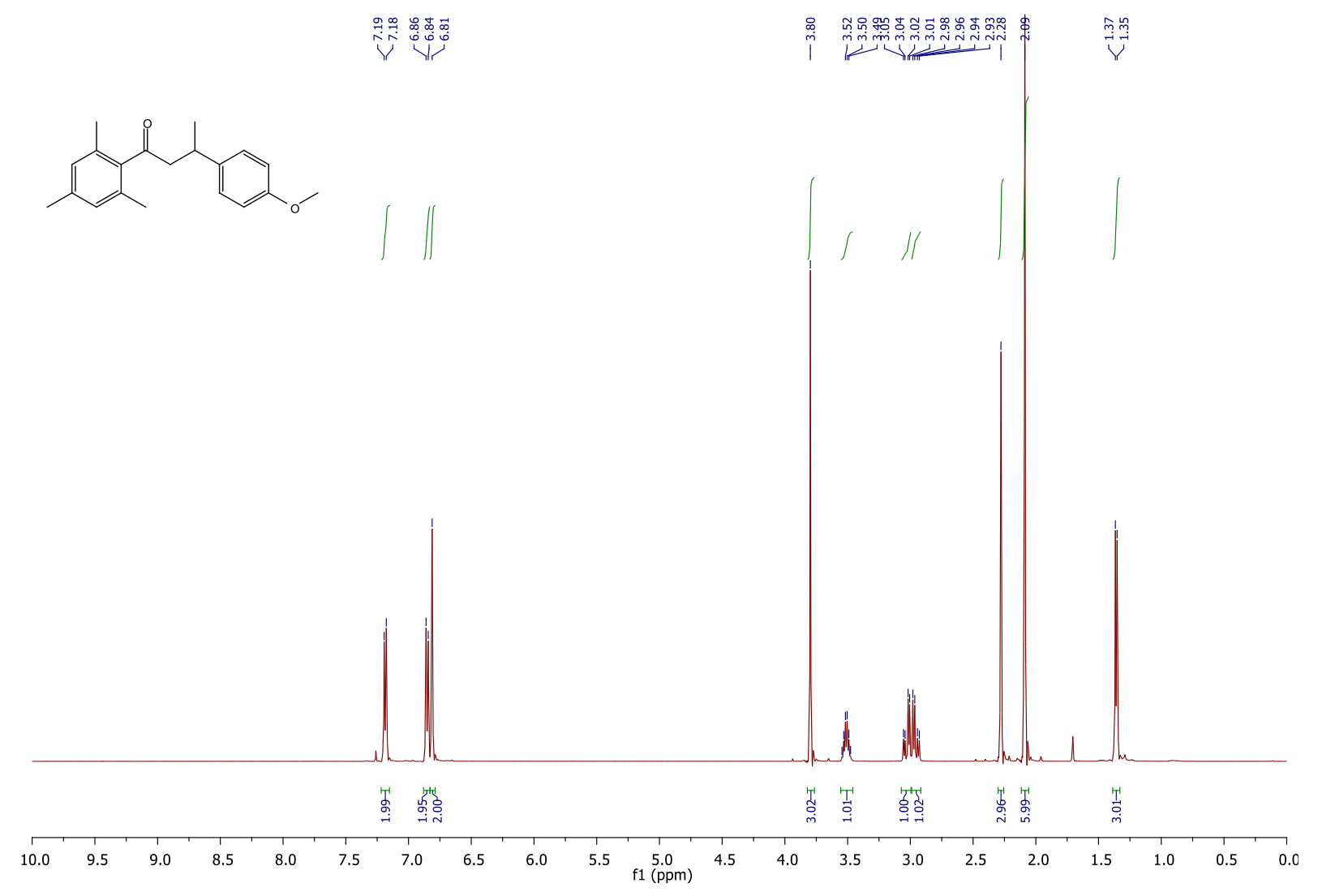

Figure S8: ${ }^{1} \mathrm{H}-\mathrm{NMR}(500 \mathrm{MHz})$ in $\mathrm{CDCl}_{3}$ of $1 \mathrm{~b}$

$\hat{i}$

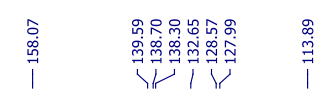

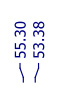

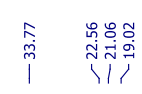

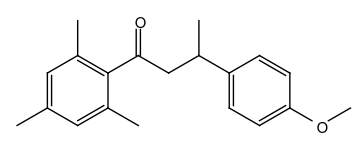
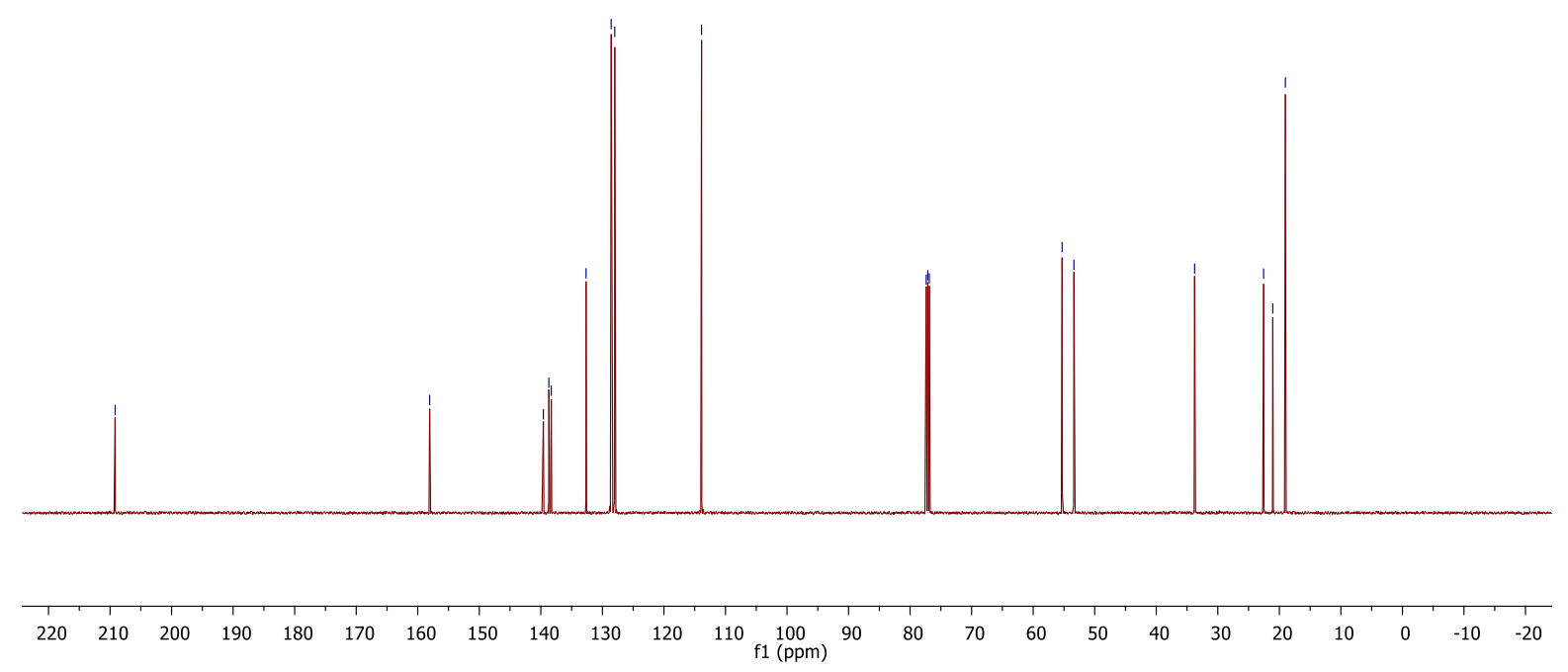

Figure S9: ${ }^{13} \mathrm{C}-\mathrm{NMR}(125 \mathrm{MHz})$ in $\mathrm{CDCl}_{3}$ of $1 \mathrm{~b}$

S-27 


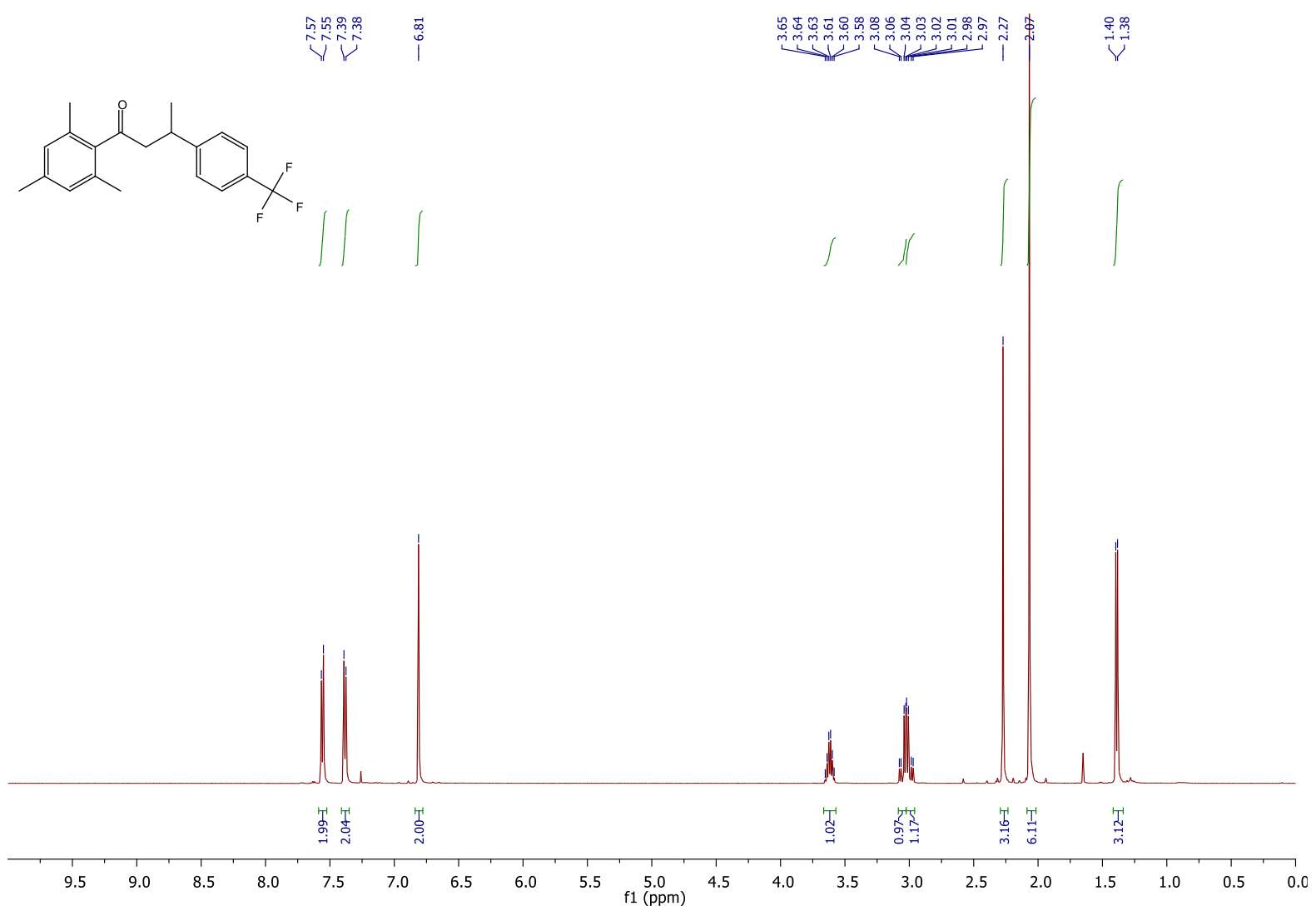

Figure S10: ${ }^{1} \mathrm{H}-\mathrm{NMR}(500 \mathrm{MHz})$ in $\mathrm{CDCl}_{3}$ of $1 \mathrm{C}$
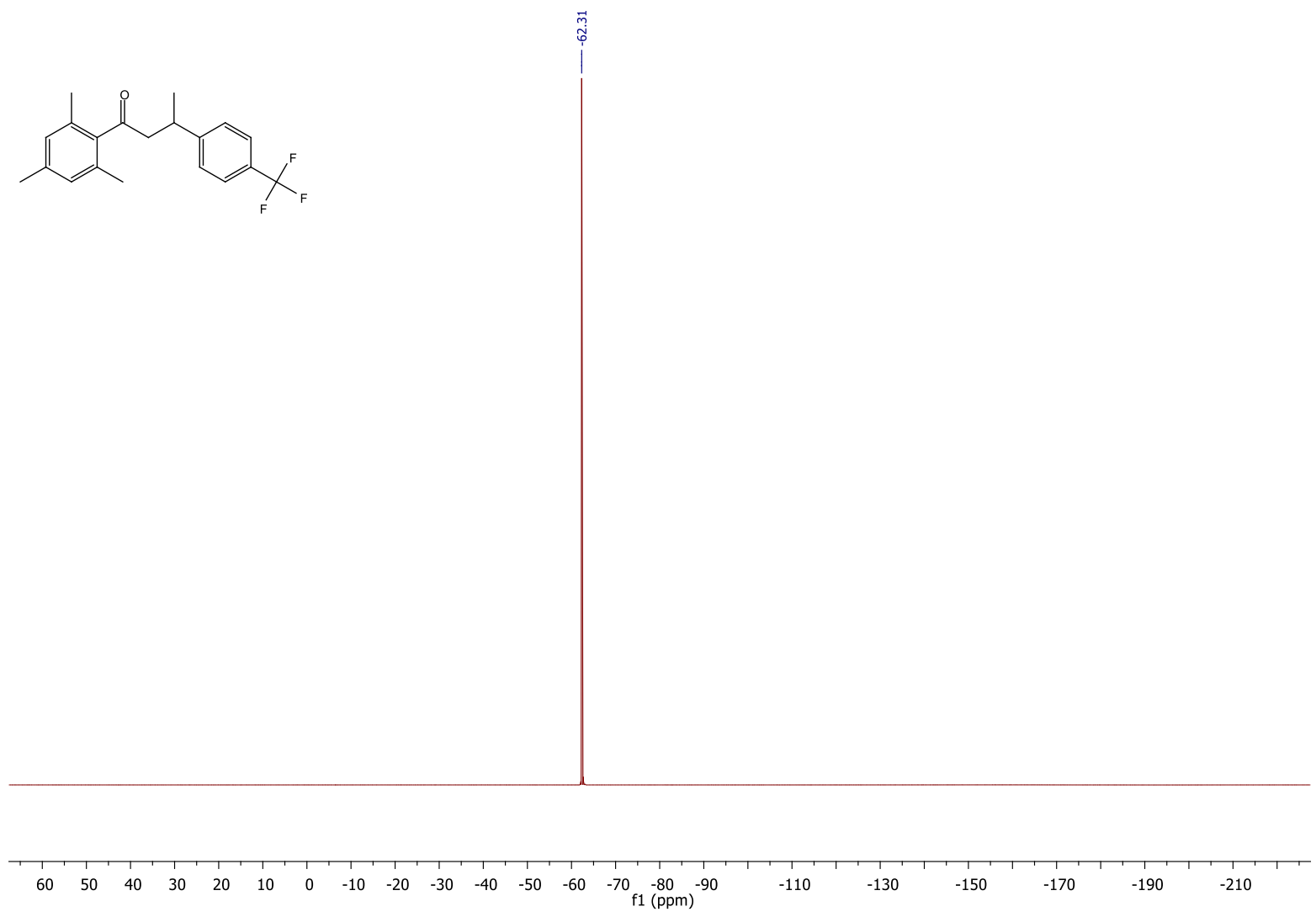

Figure S11: ${ }^{19} \mathrm{~F}-\mathrm{NMR}(500 \mathrm{MHz})$ in $\mathrm{CDCl}_{3}$ of $1 \mathrm{C}$

S-28 


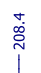

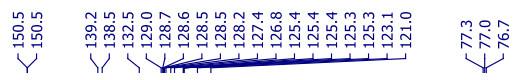

î.
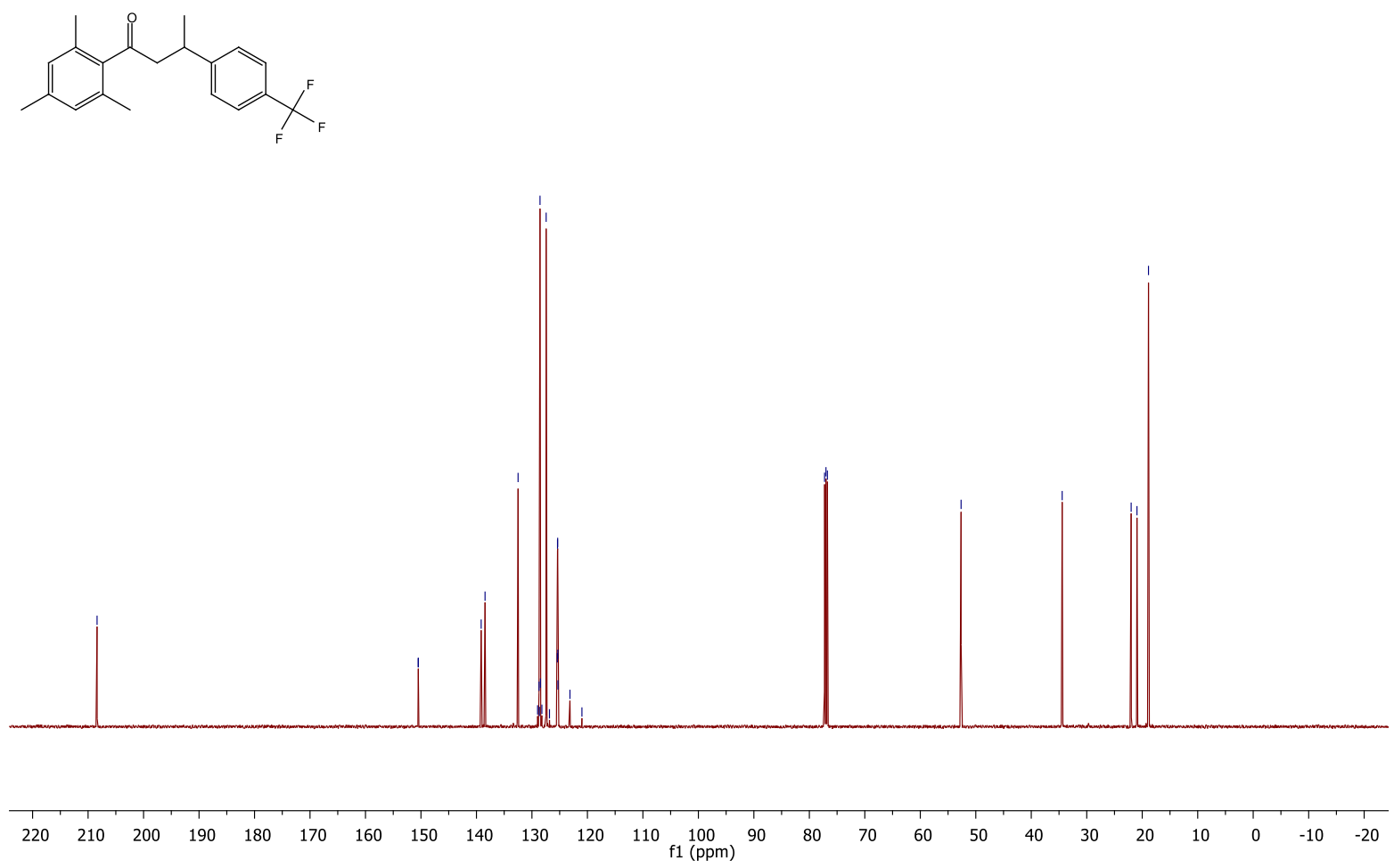

Figure S12: ${ }^{13} \mathrm{C}-\mathrm{NMR}(125 \mathrm{MHz})$ in $\mathrm{CDCl}_{3}$ of $1 \mathrm{C}$

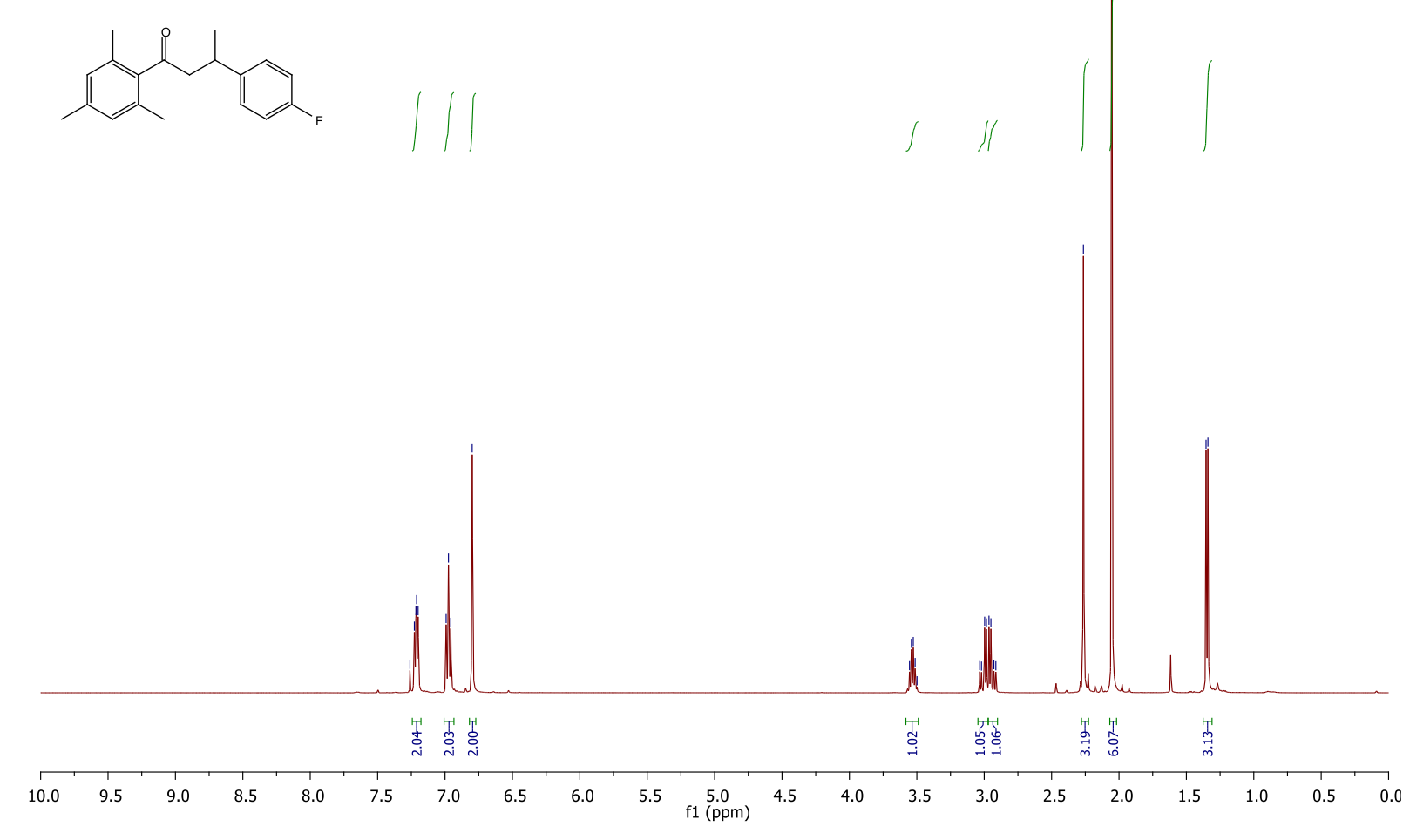

ำ

Yे.

Figure S13: ${ }^{1} \mathrm{H}-\mathrm{NMR}(500 \mathrm{MHz})$ in $\mathrm{CDCl}_{3}$ of $1 \mathrm{~d}$

S-29 
$\stackrel{8}{\stackrel{\overbrace{}}{i}}$
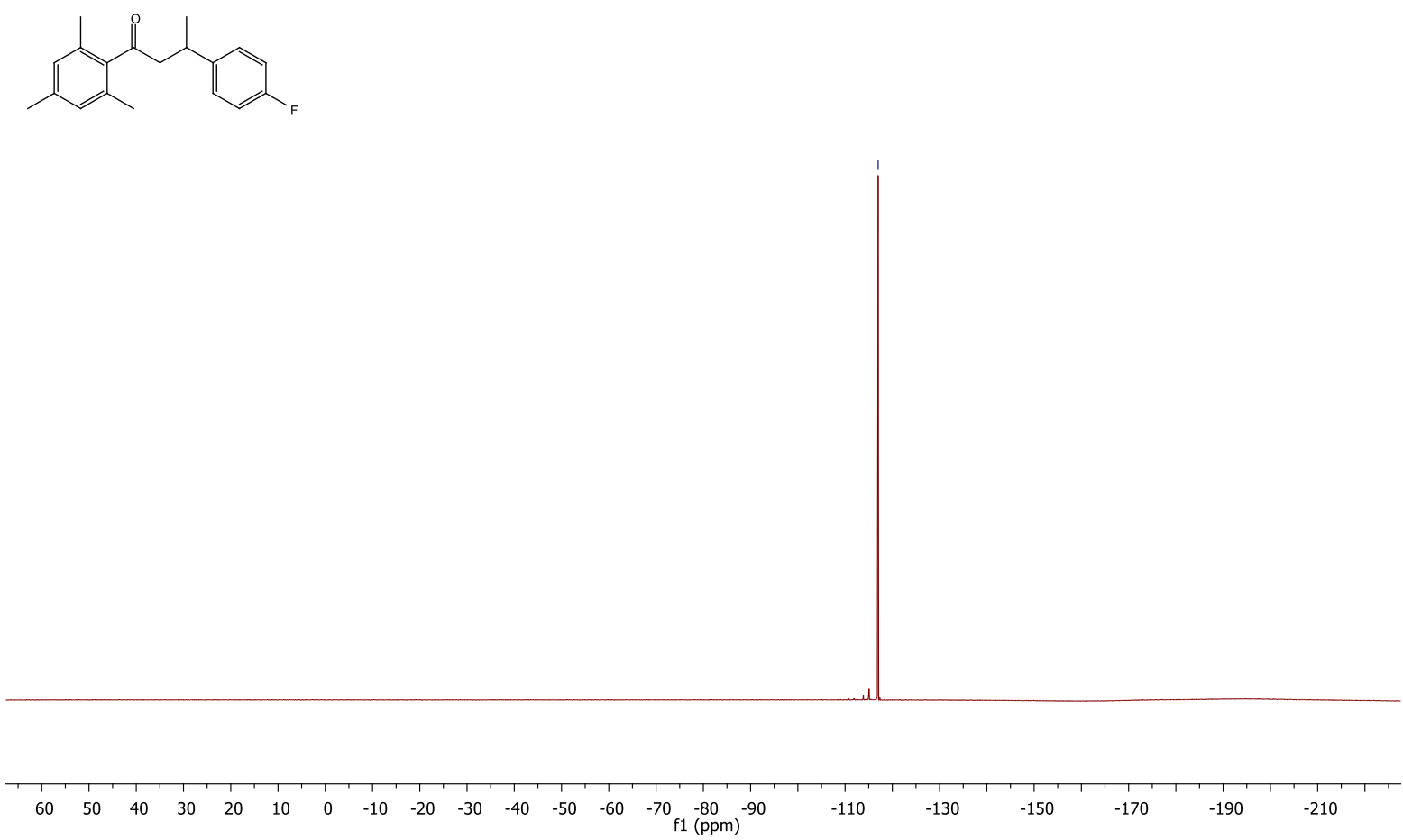

Figure S14: ${ }^{19} \mathrm{~F}-\mathrm{NMR}(500 \mathrm{MHz})$ in $\mathrm{CDCl}_{3}$ of $1 \mathrm{~d}$
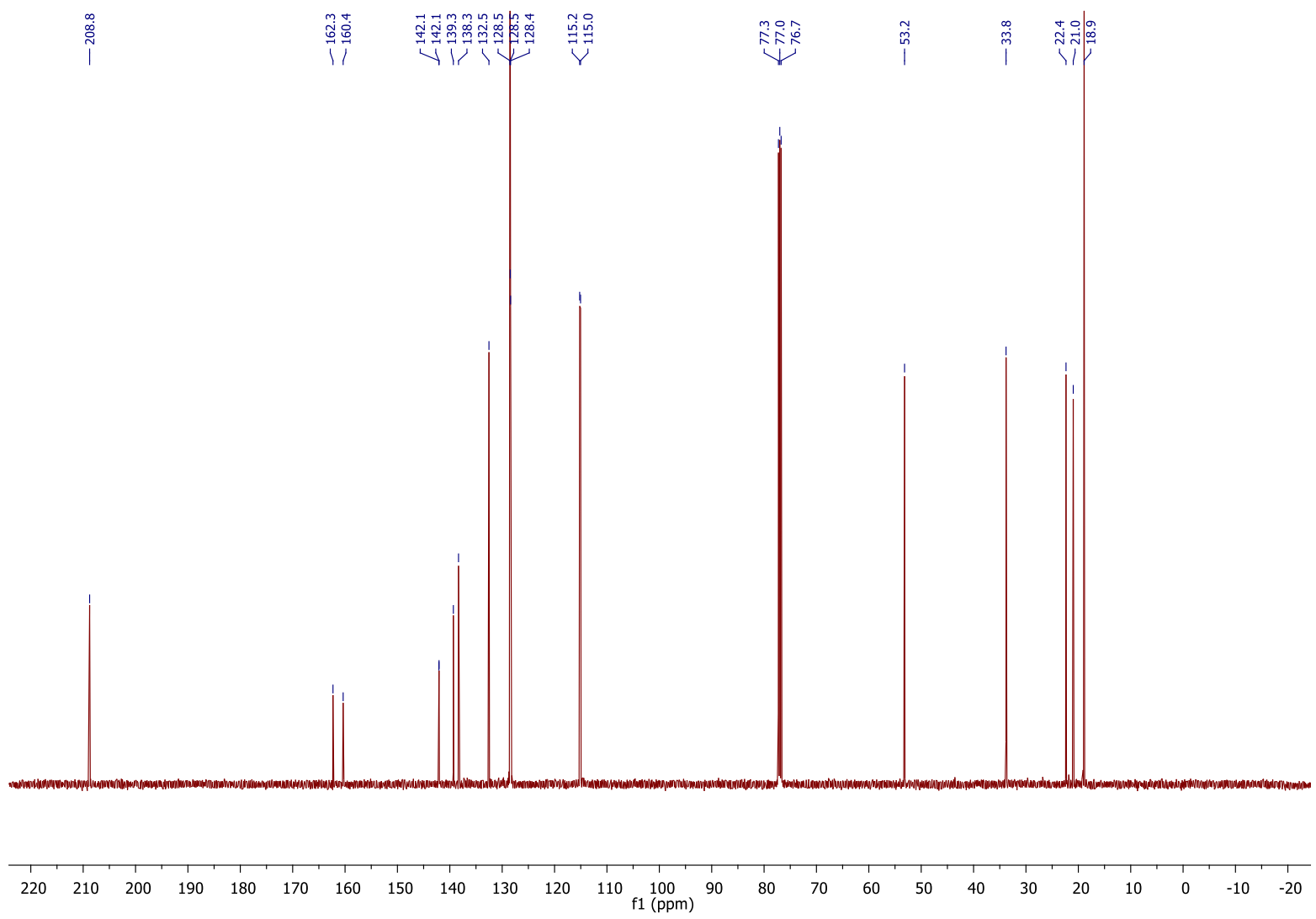

Figure S15: ${ }^{13} \mathrm{C}$-NMR (125 MHz) in $\mathrm{CDCl}_{3}$ of $1 \mathrm{~d}$

S-30 


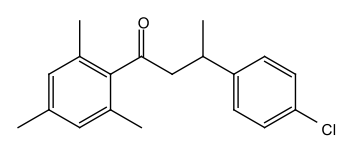

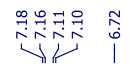

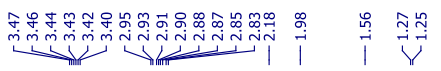

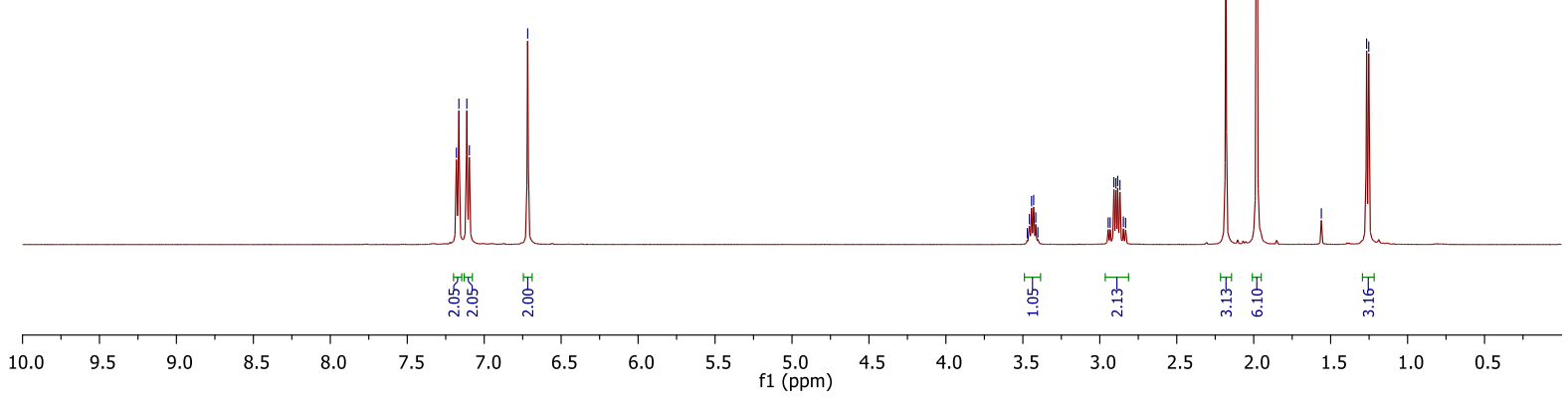

Figure S16: ${ }^{1} \mathrm{H}-\mathrm{NMR}(500 \mathrm{MHz})$ in $\mathrm{CDCl}_{3}$ of $1 \mathrm{e}$

@o

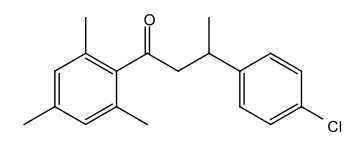

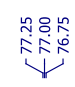

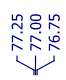
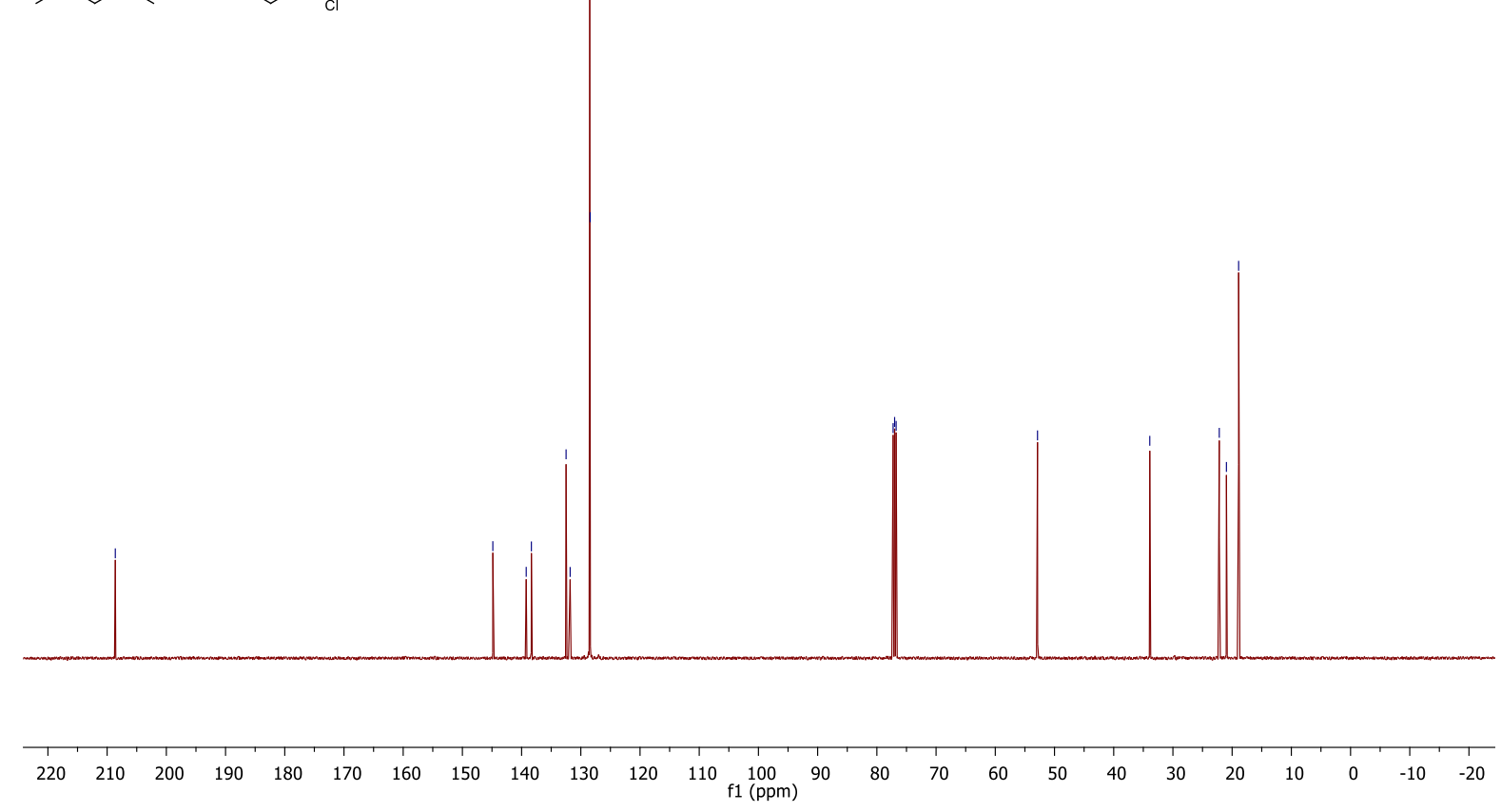

Figure S17: ${ }^{13} \mathrm{C}-\mathrm{NMR}(125 \mathrm{MHz})$ in $\mathrm{CDCl}_{3}$ of $1 \mathrm{e}$

S-31 


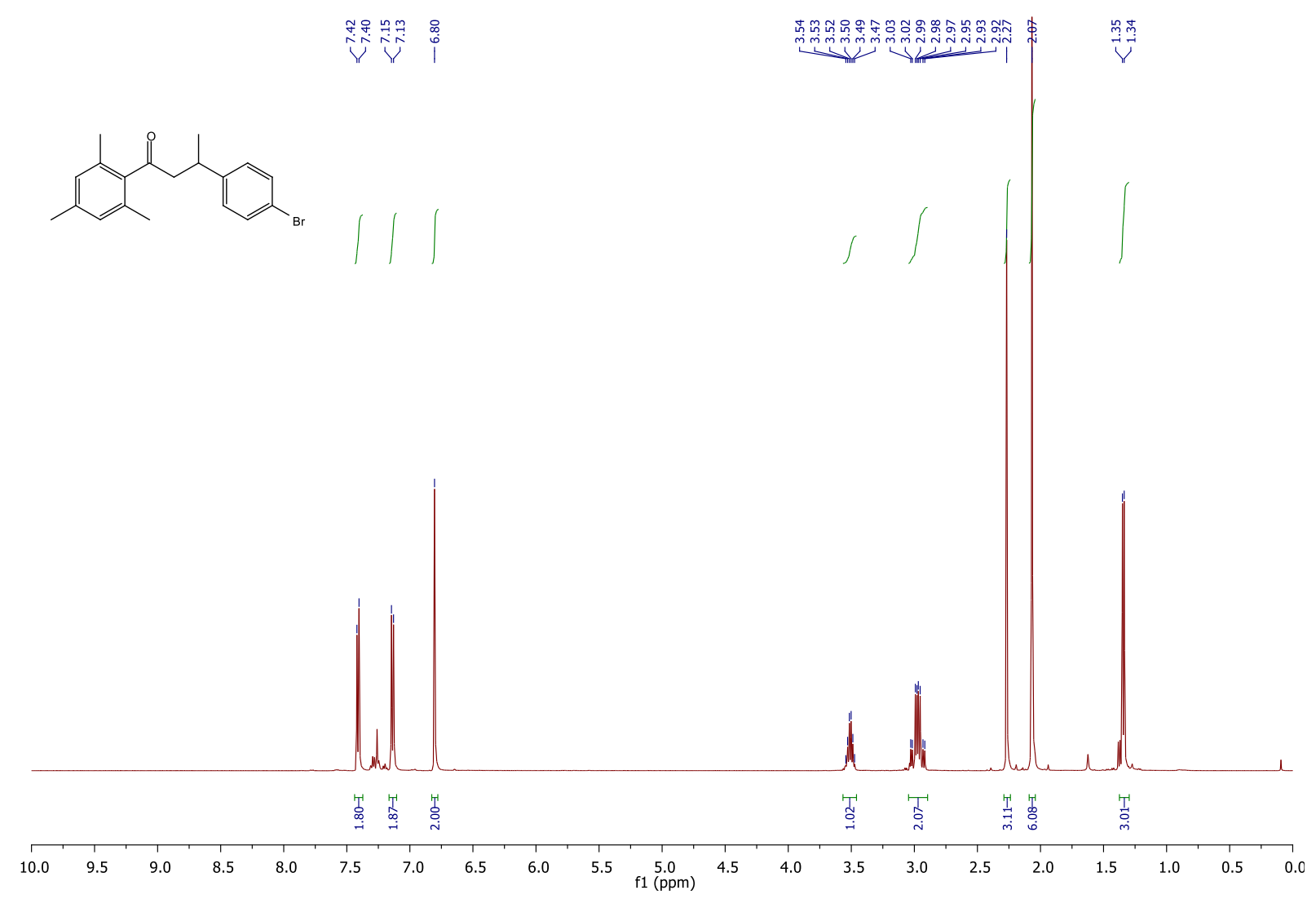

Figure S18: ${ }^{1} \mathrm{H}-\mathrm{NMR}(500 \mathrm{MHz})$ in $\mathrm{CDCl}_{3}$ of $1 \mathrm{f}$
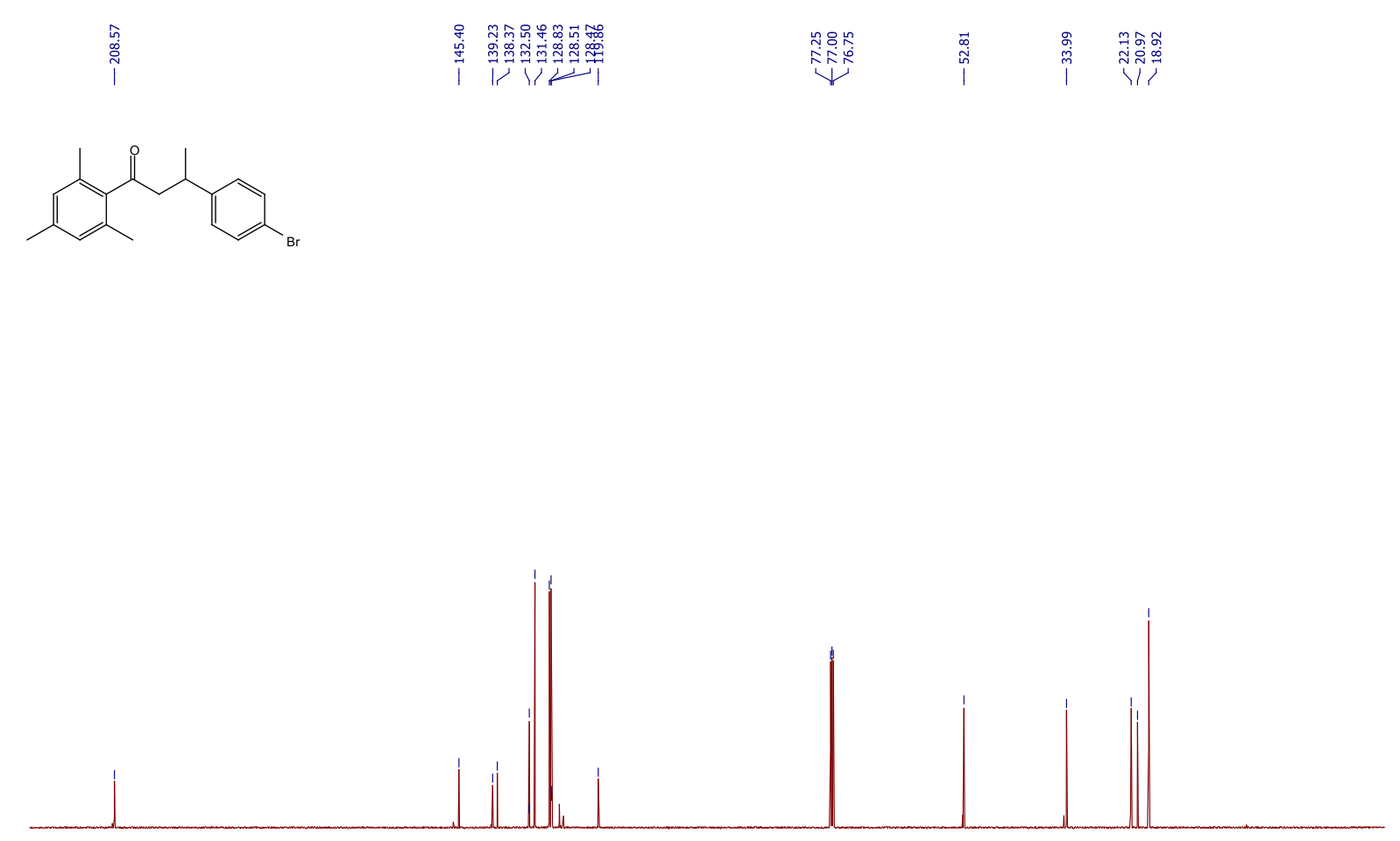

$\begin{array}{llllllllllllllllllllllllllllllllllllllll}220 & 210 & 200 & 190 & 180 & 170 & 160 & 150 & 140 & 130 & 120 & 110 & 100 & 90 & 80 & 70 & 60 & 50 & 40 & 30 & 20 & 10 & 0 & -10 & -20\end{array}$

Figure S19: ${ }^{13} \mathrm{C}$-NMR (125 MHz) in $\mathrm{CDCl}_{3}$ of $1 \mathrm{f}$ 


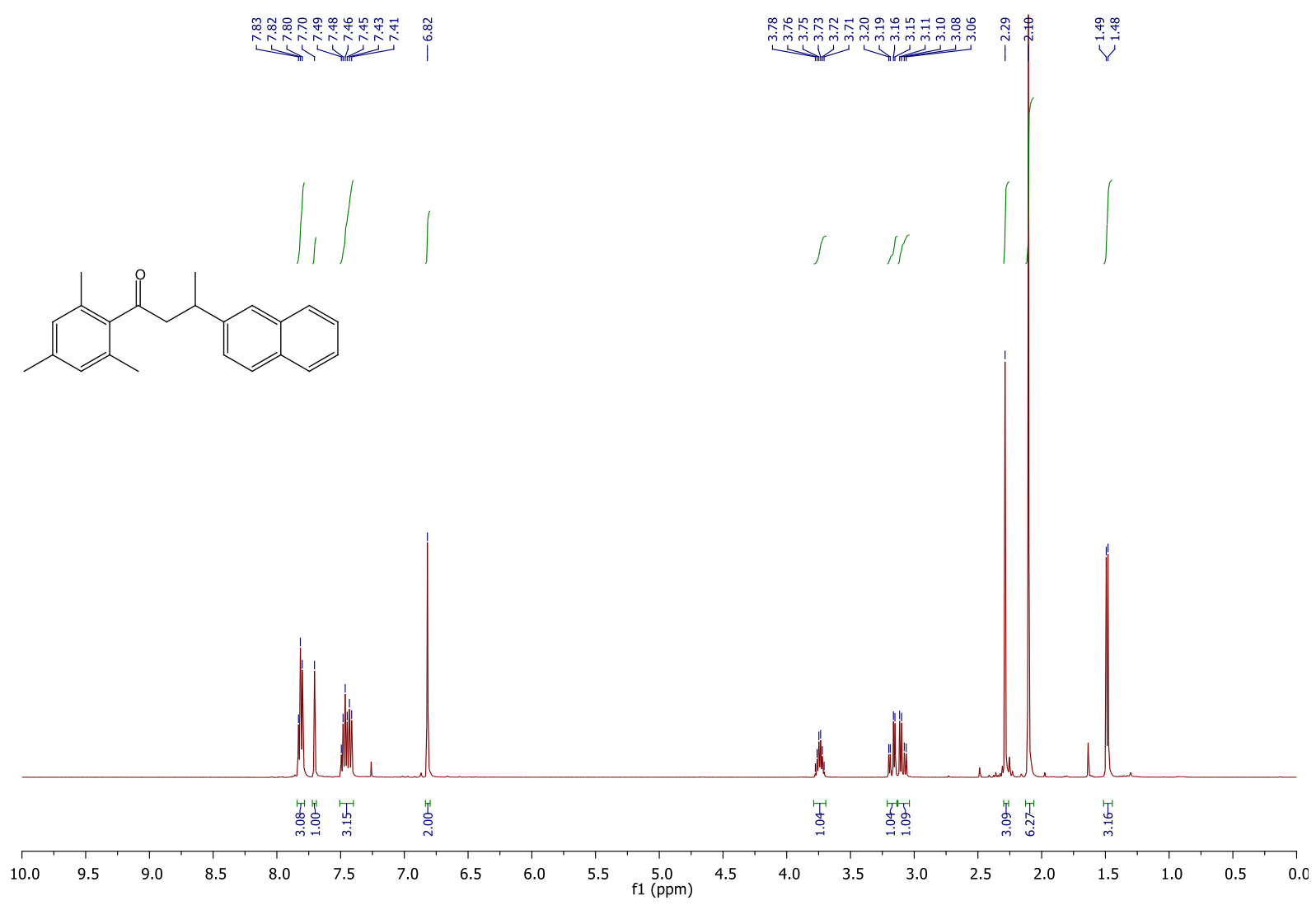

Figure S20: ${ }^{1} \mathrm{H}-\mathrm{NMR}(500 \mathrm{MHz})$ in $\mathrm{CDCl}_{3}$ of $1 \mathrm{~g}$
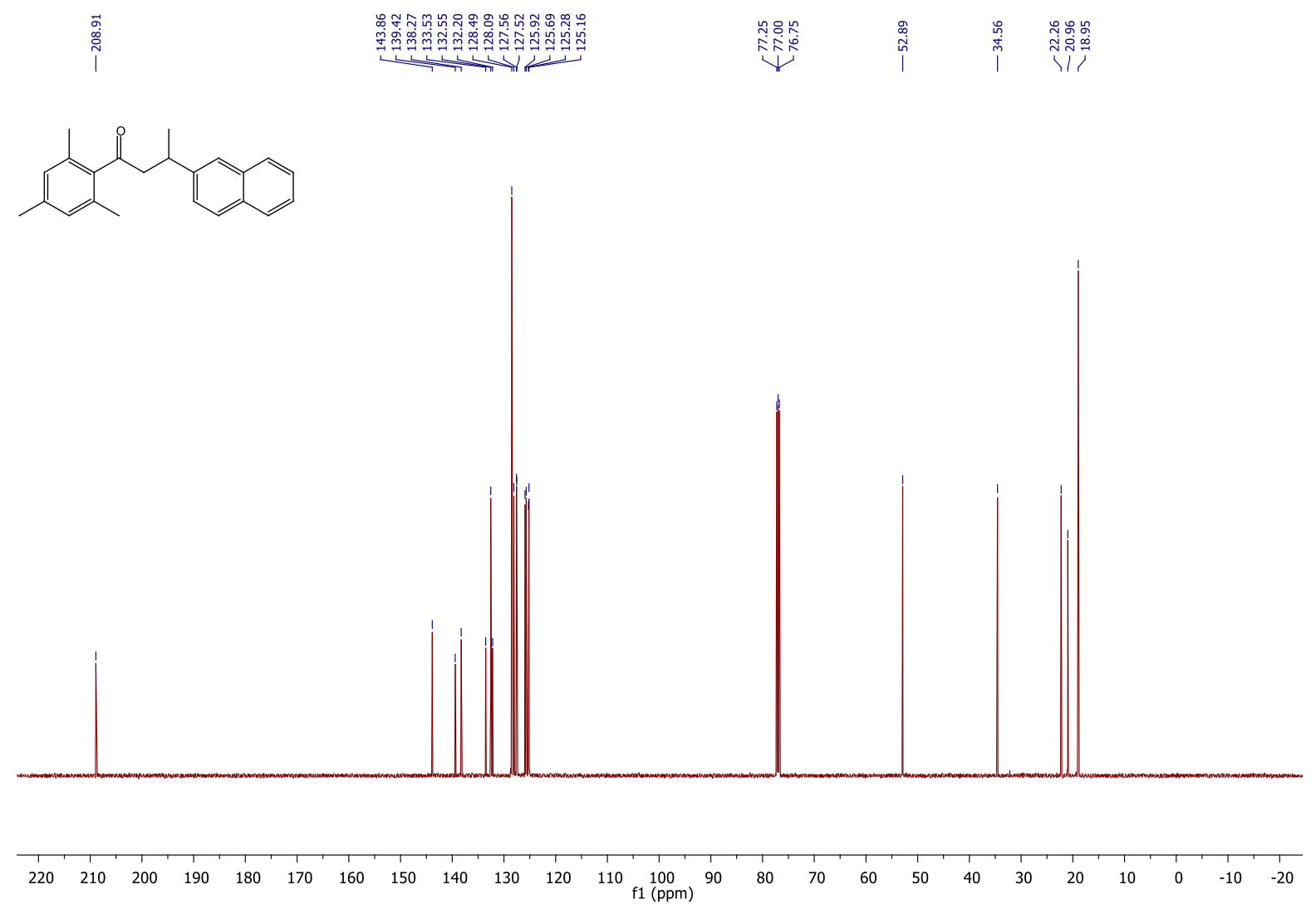

Figure S21: ${ }^{13} \mathrm{C}-\mathrm{NMR}(125 \mathrm{MHz})$ in $\mathrm{CDCl}_{3}$ of $1 \mathrm{~g}$

S-33 


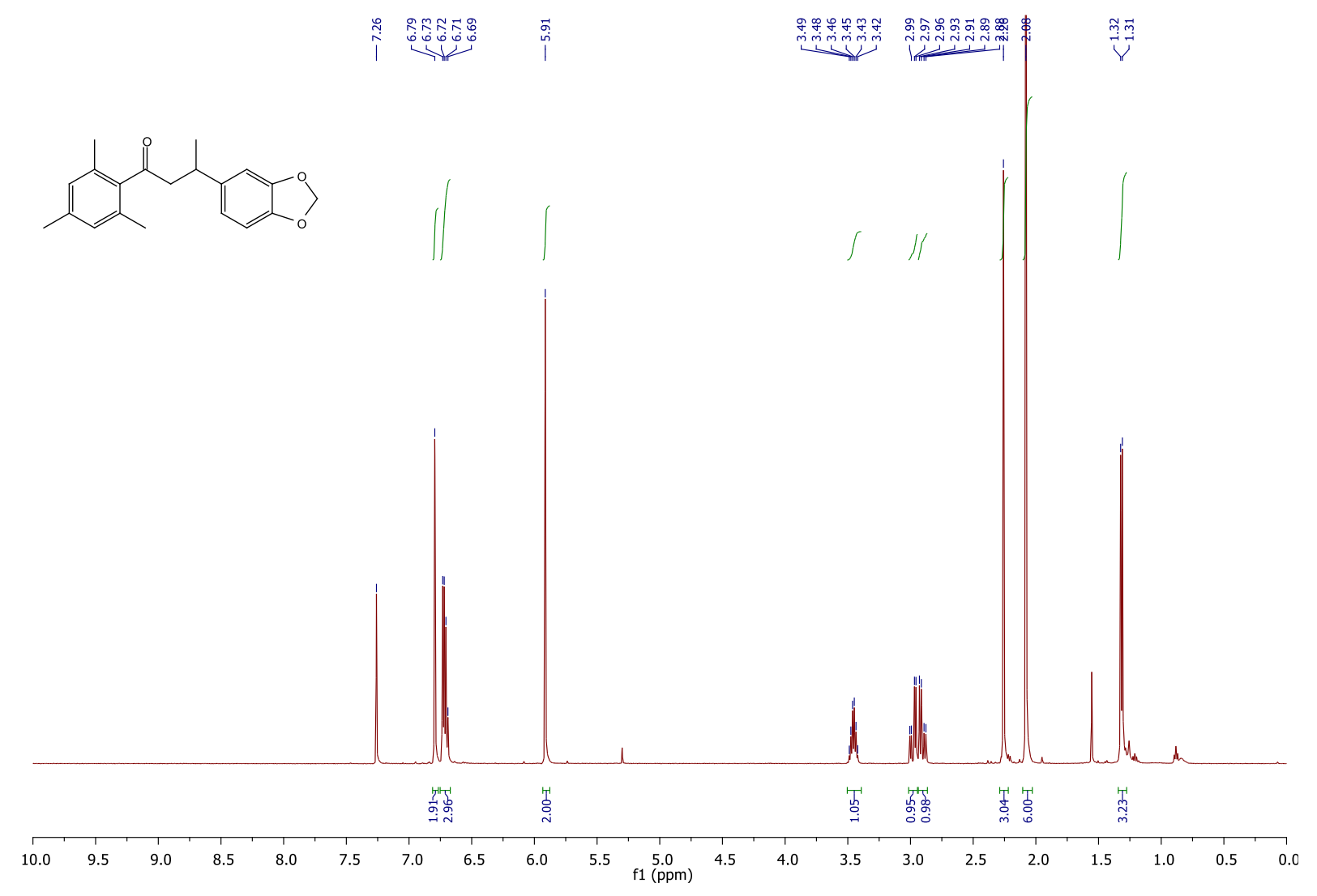

Figure S22: ${ }^{1} \mathrm{H}-\mathrm{NMR}(500 \mathrm{MHz})$ in $\mathrm{CDCl}_{3}$ of $1 \mathrm{~h}$
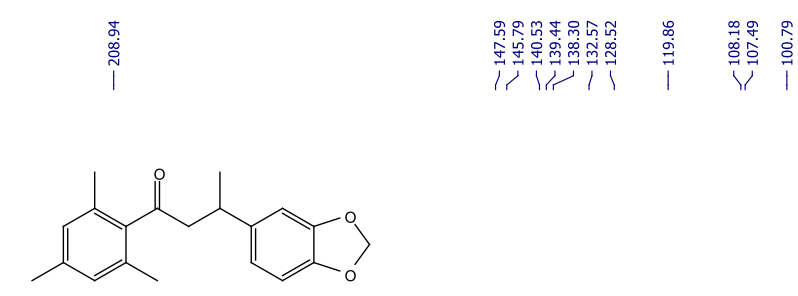

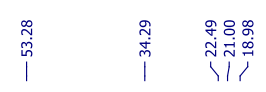

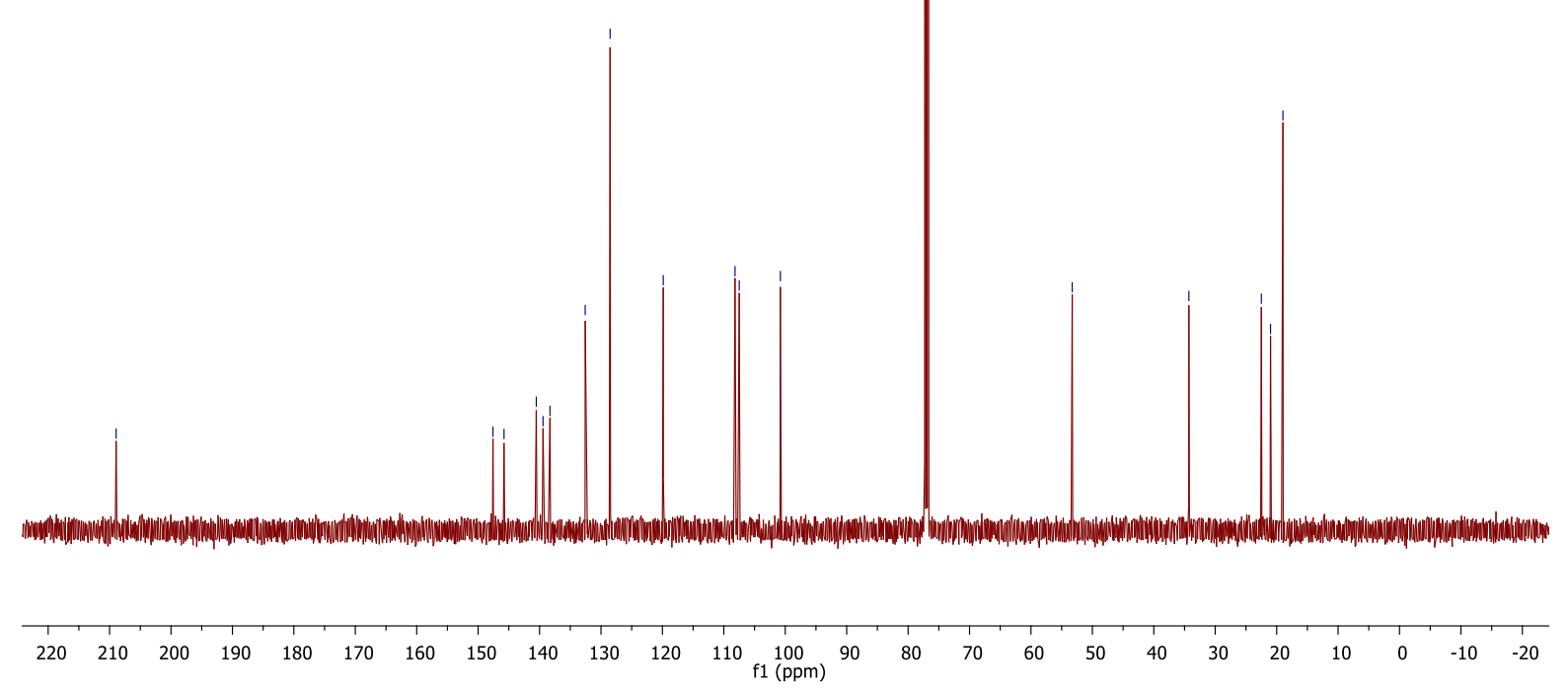

Figure S23: ${ }^{13} \mathrm{C}-\mathrm{NMR}(125 \mathrm{MHz})$ in $\mathrm{CDCl}_{3}$ of $1 \mathrm{~h}$

S-34 


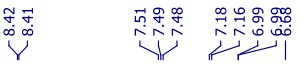

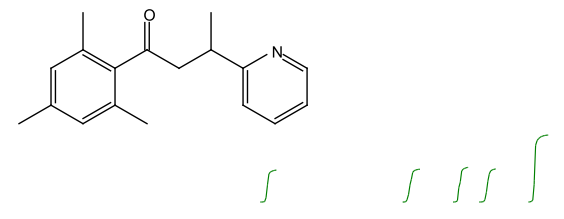

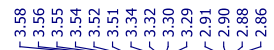

ํำ

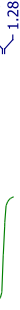

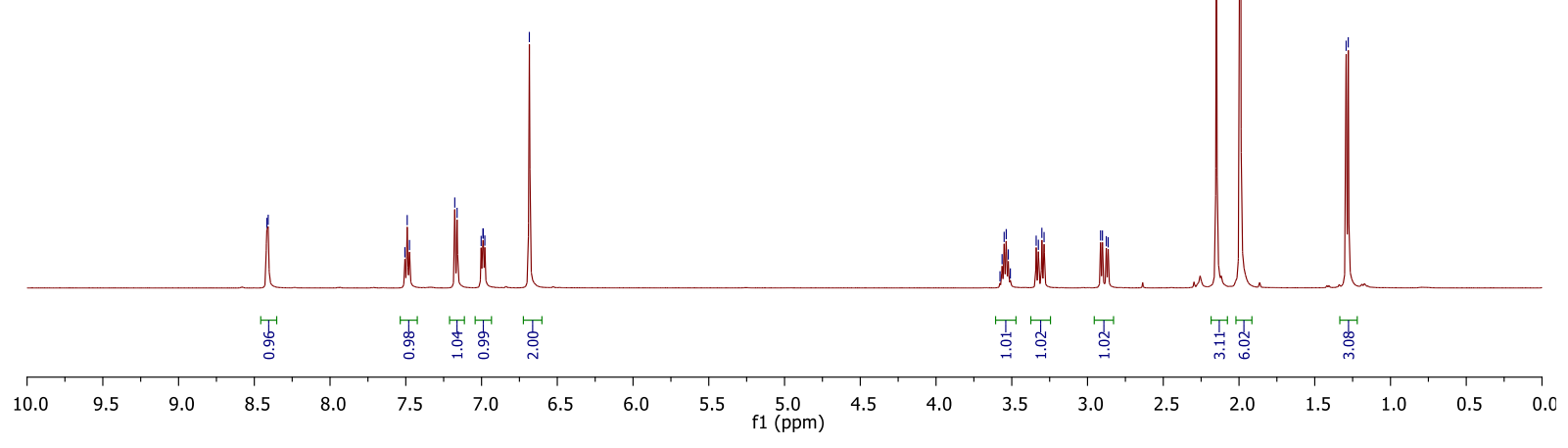

Figure S24: ${ }^{1} \mathrm{H}-\mathrm{NMR}(500 \mathrm{MHz})$ in $\mathrm{CDCl}_{3}$ of $1 \mathrm{i}$

월

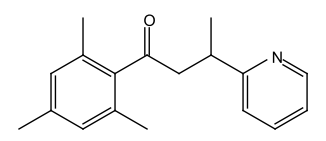

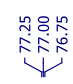

$\stackrel{\widetilde{\omega}}{\stackrel{\omega}{~}}$
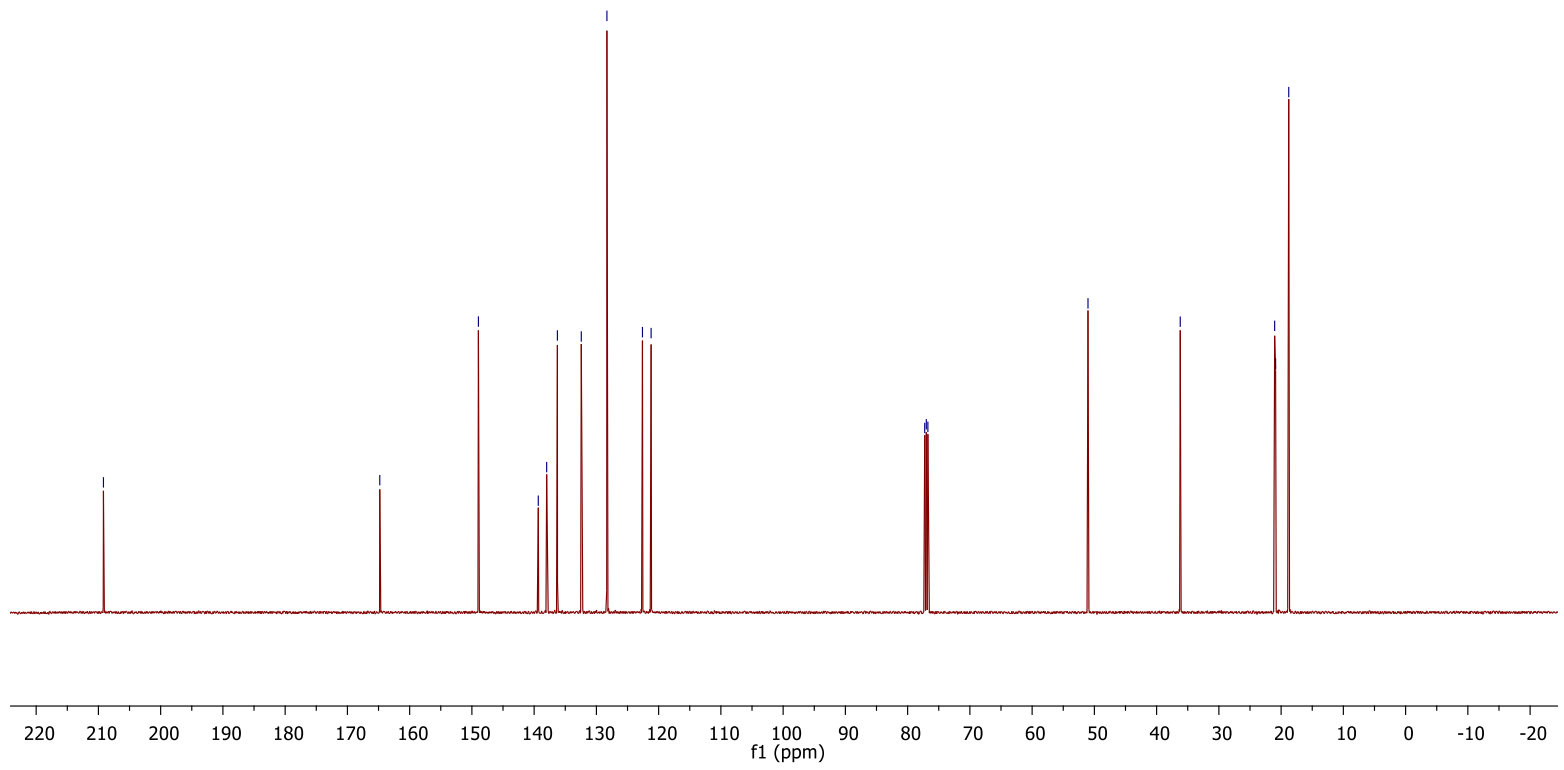

Figure S25: ${ }^{13} \mathrm{C}$-NMR (125 MHz) in $\mathrm{CDCl}_{3}$ of $1 \mathrm{i}$

S-35 


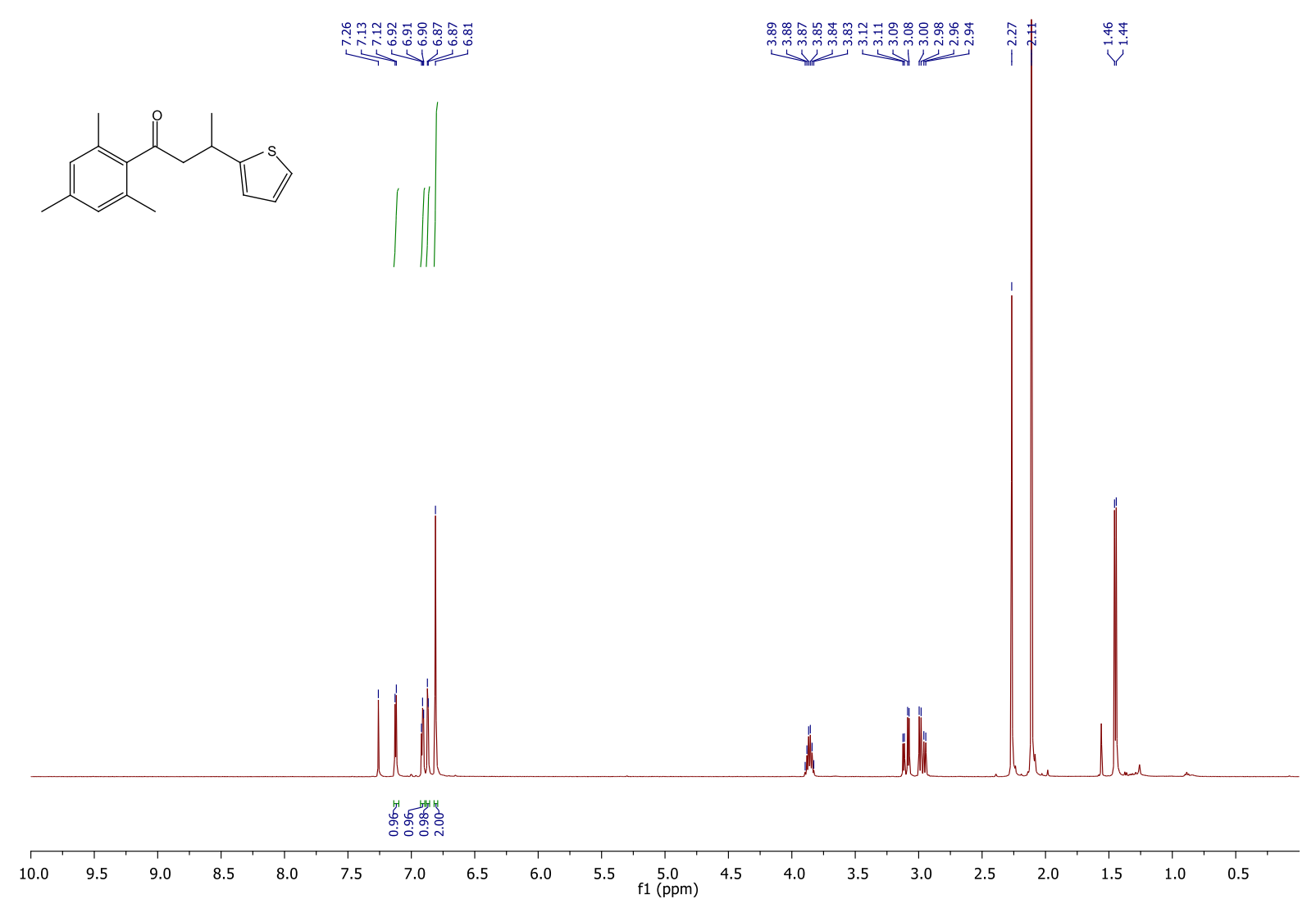

Figure S26: ${ }^{1} \mathrm{H}-\mathrm{NMR}(500 \mathrm{MHz})$ in $\mathrm{CDCl}_{3}$ of $1 \mathrm{j}$

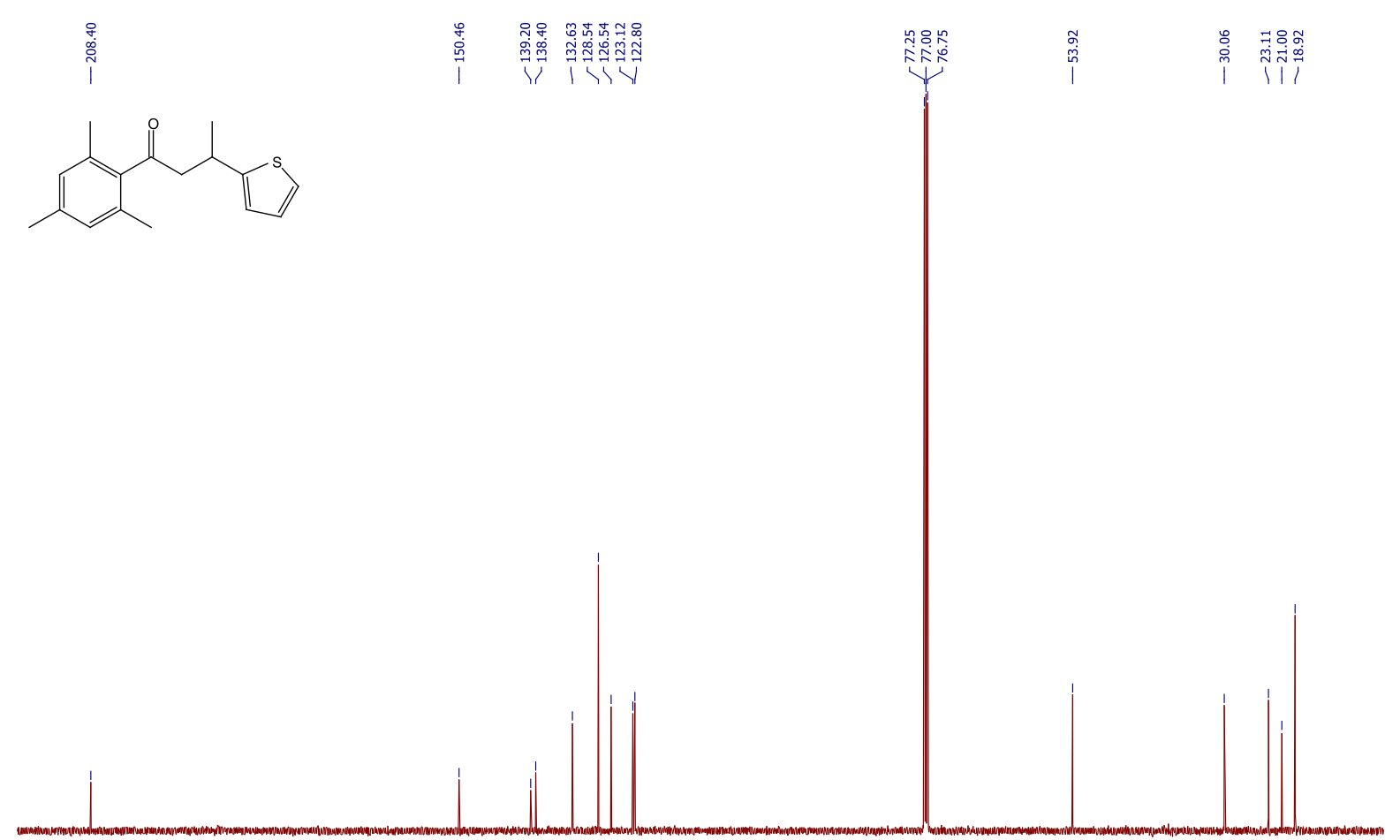

$\begin{array}{lllllllllllllllllllllllll}210 & 200 & 190 & 180 & 170 & 160 & 150 & 140 & 130 & 120 & 110 & 100 & 90 & 80 & 70 & 60 & 50 & 40 & 30 & 20 & 10\end{array}$

Figure S27: ${ }^{13} \mathrm{C}-\mathrm{NMR}(125 \mathrm{MHz})$ in $\mathrm{CDCl}_{3}$ of $1 \mathrm{j}$ 


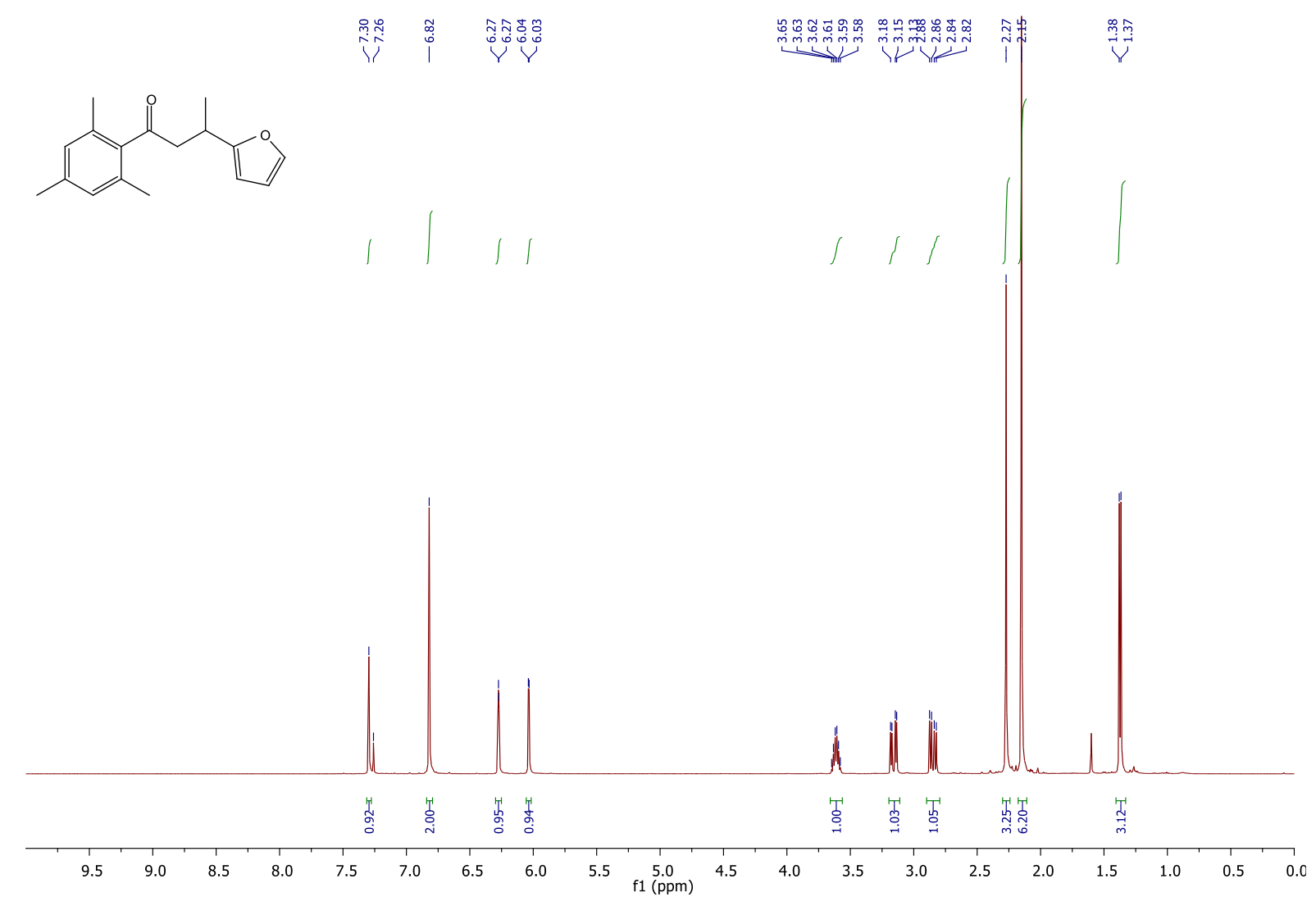

Figure S28: ${ }^{1} \mathrm{H}-\mathrm{NMR}(500 \mathrm{MHz})$ in $\mathrm{CDCl}_{3}$ of $1 \mathrm{k}$
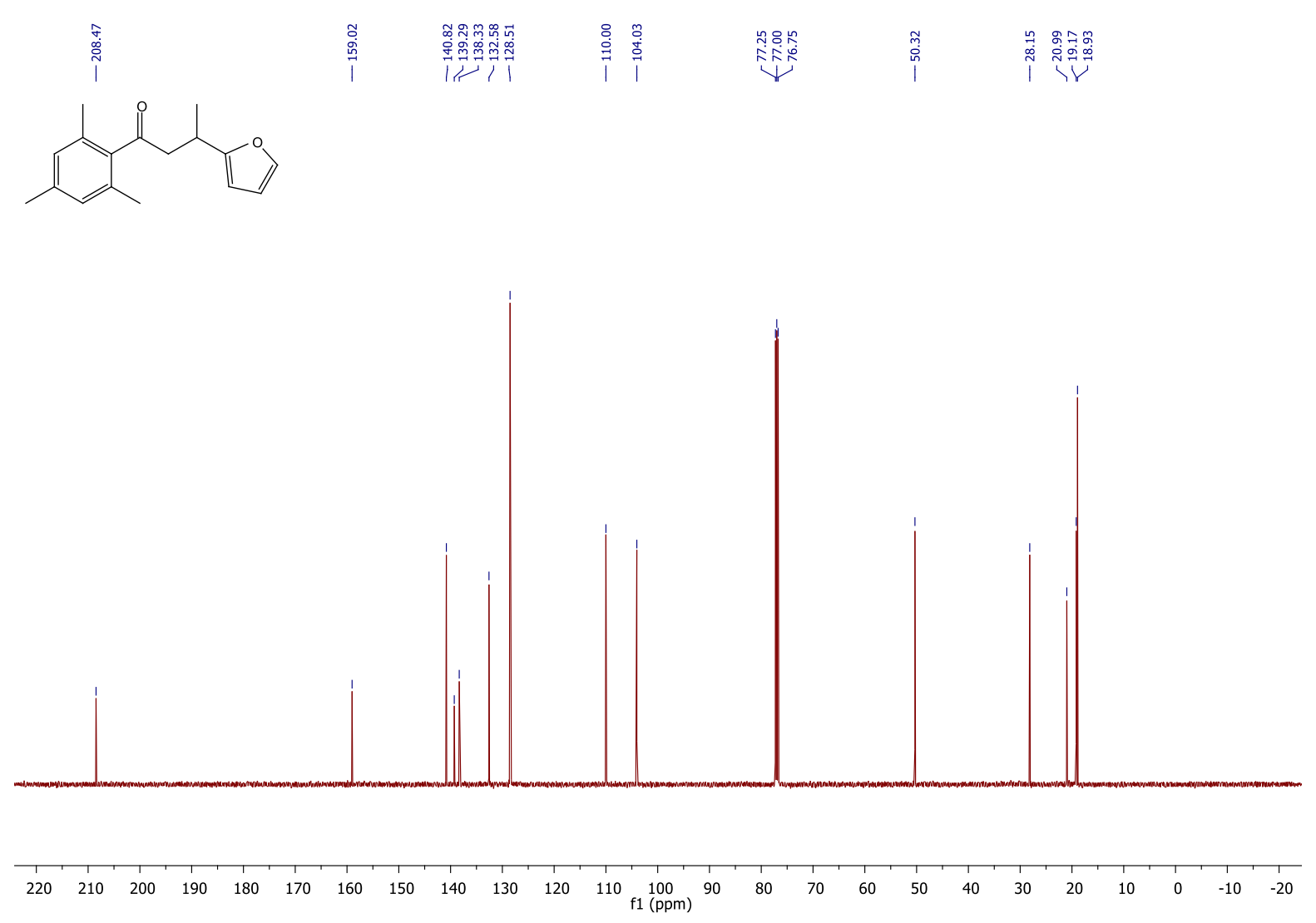

Figure S29: ${ }^{13} \mathrm{C}-\mathrm{NMR}(125 \mathrm{MHz})$ in $\mathrm{CDCl}_{3}$ of $1 \mathrm{k}$ 


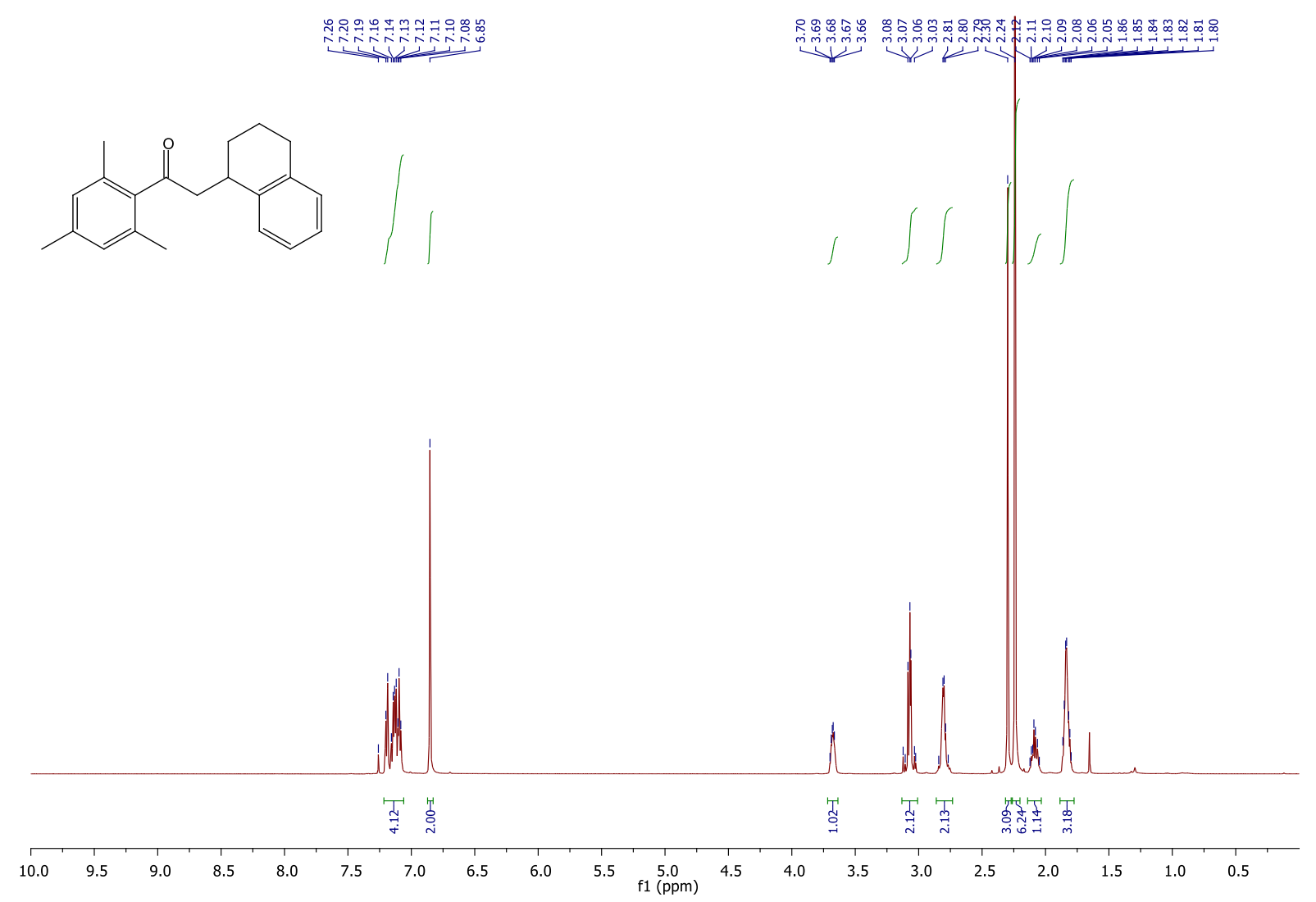

Figure S30: ${ }^{1} \mathrm{H}-\mathrm{NMR}(500 \mathrm{MHz})$ in $\mathrm{CDCl}_{3}$ of 11

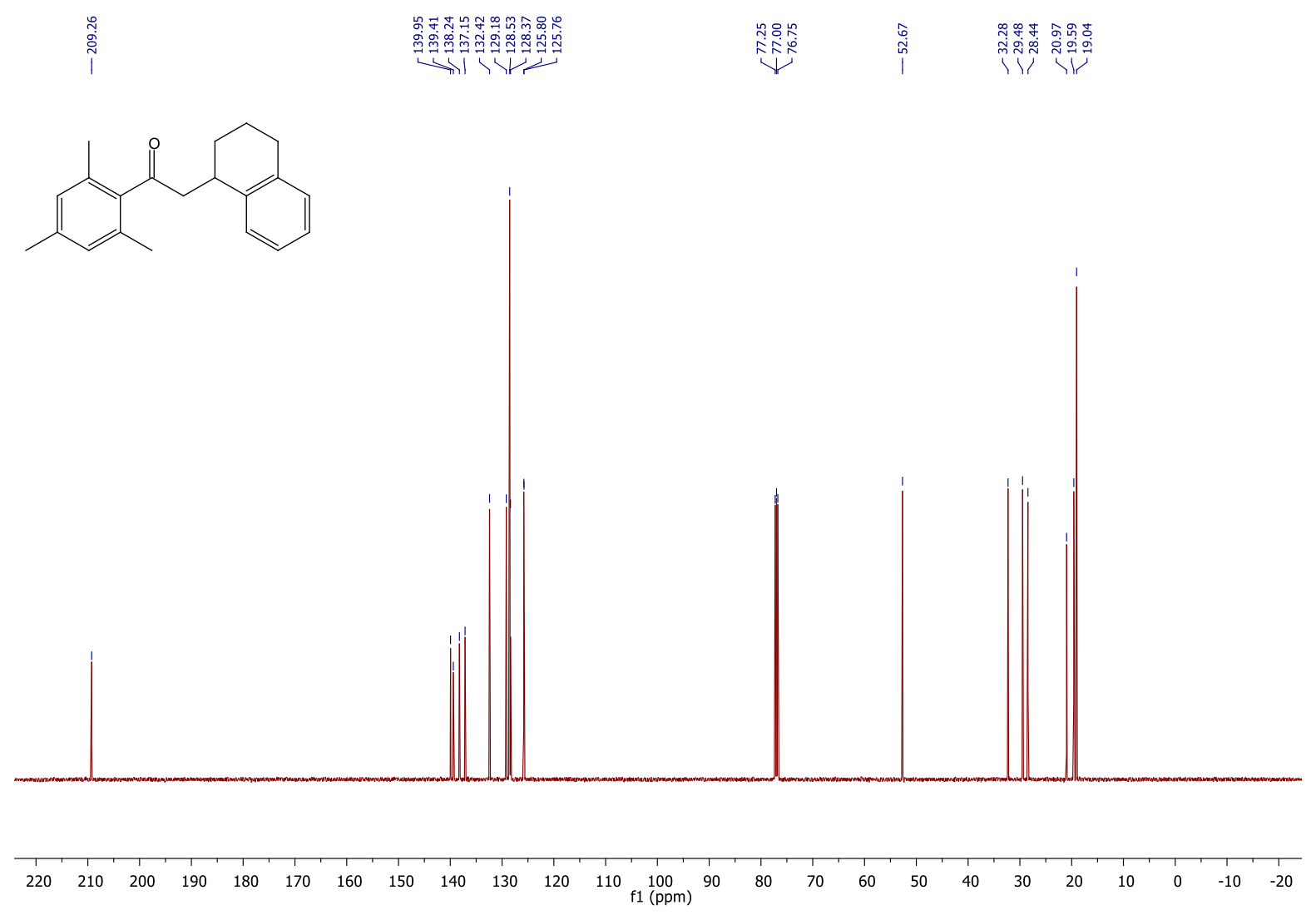

Figure S31: ${ }^{13} \mathrm{C}$-NMR (125 MHz) in $\mathrm{CDCl}_{3}$ of 1 I 


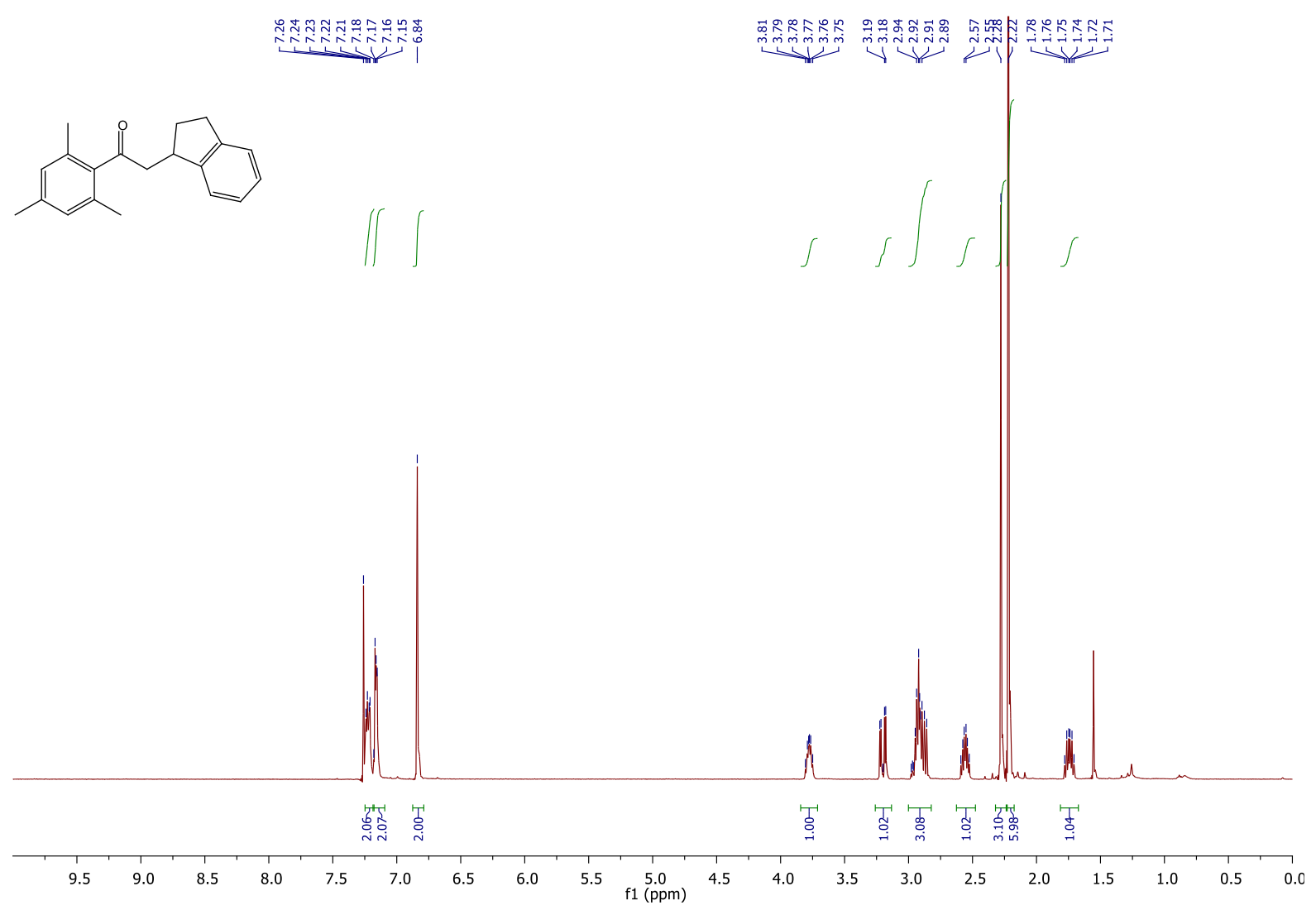

Figure S32: ${ }^{1} \mathrm{H}-\mathrm{NMR}(500 \mathrm{MHz})$ in $\mathrm{CDCl}_{3}$ of $1 \mathrm{~m}$
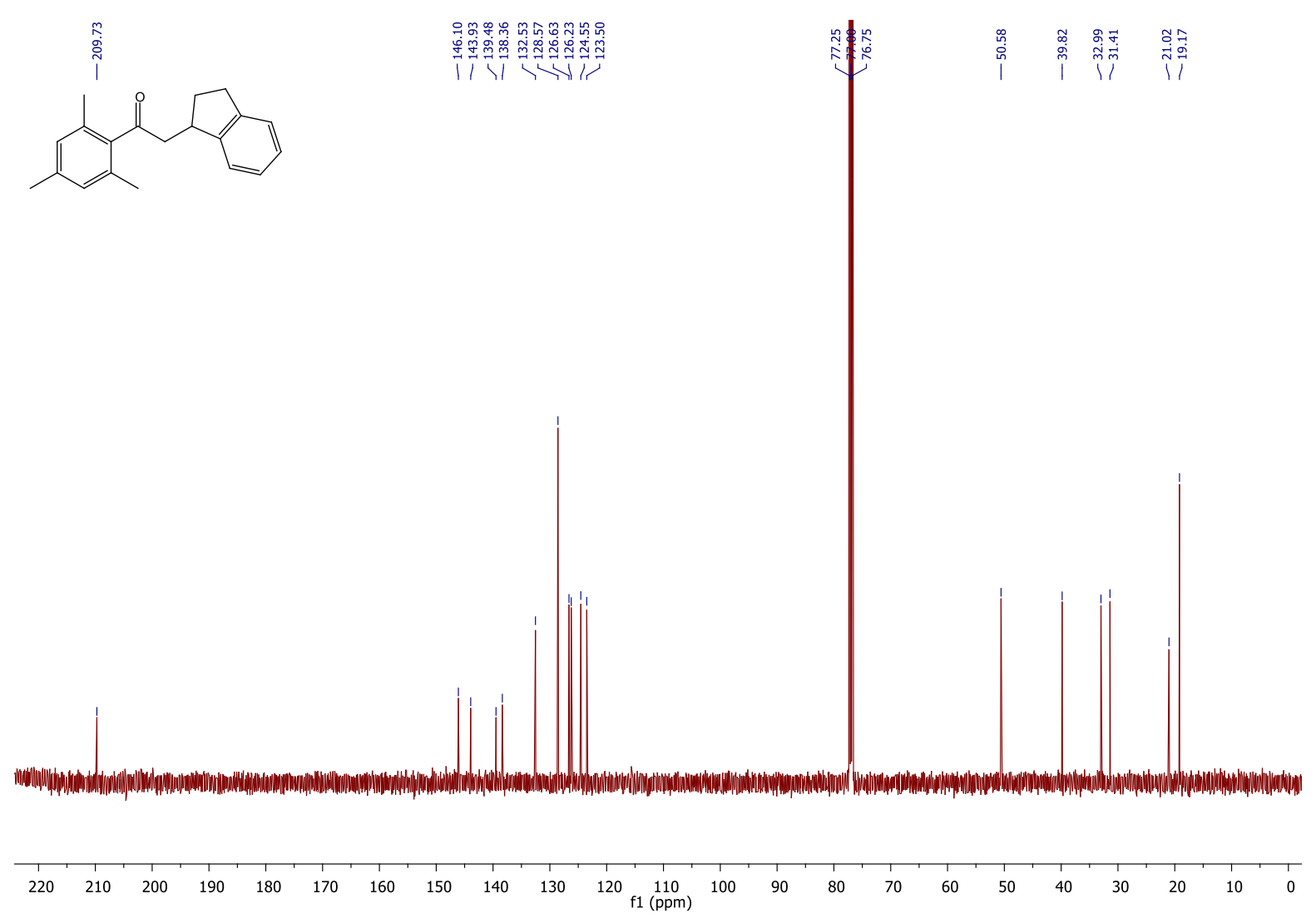

Figure S33: ${ }^{13} \mathrm{C}-\mathrm{NMR}(125 \mathrm{MHz})$ in $\mathrm{CDCl}_{3}$ of $1 \mathrm{~m}$ 

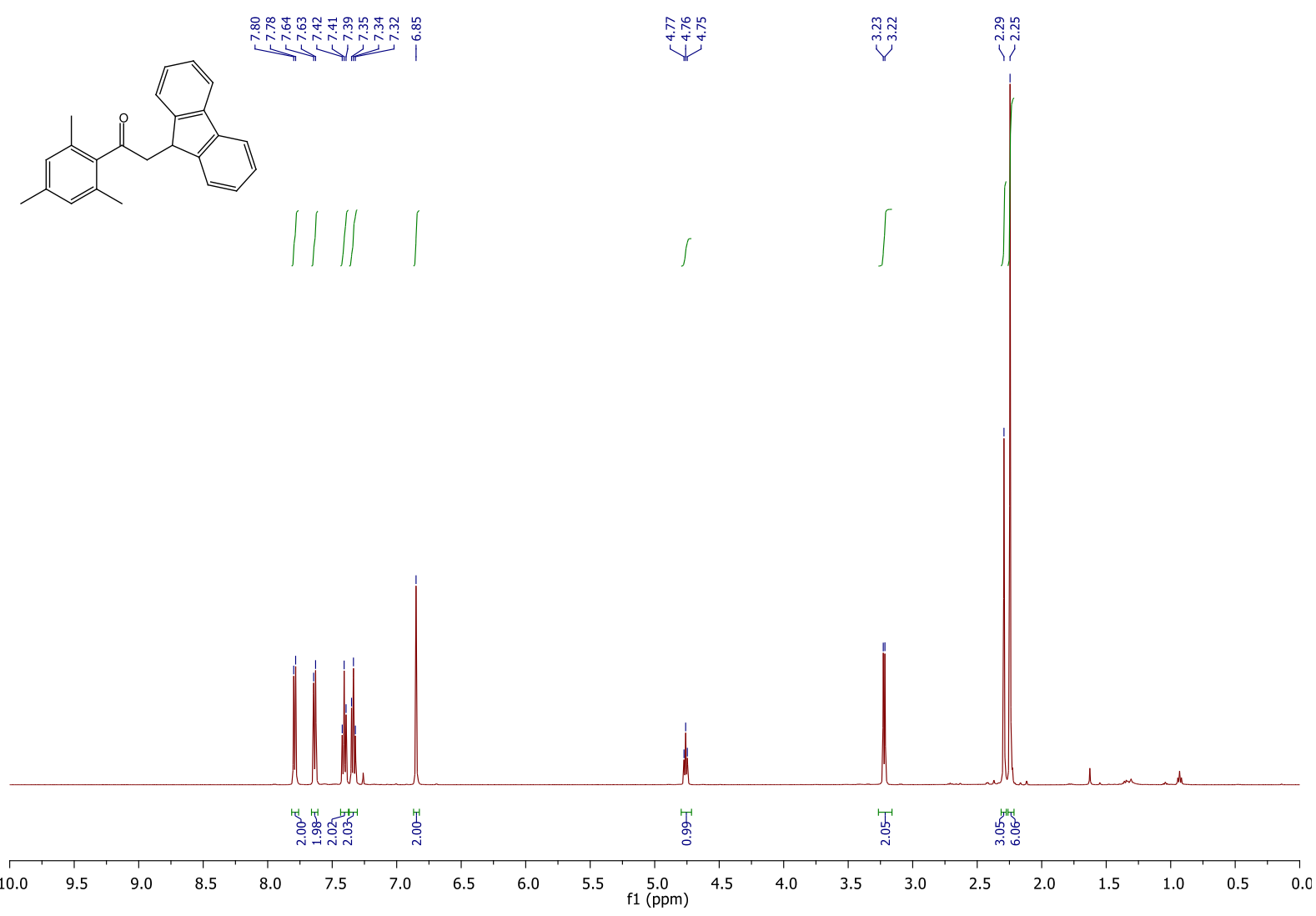

Figure S34: ${ }^{1} \mathrm{H}-\mathrm{NMR}(500 \mathrm{MHz})$ in $\mathrm{CDCl}_{3}$ of $1 \mathrm{n}$
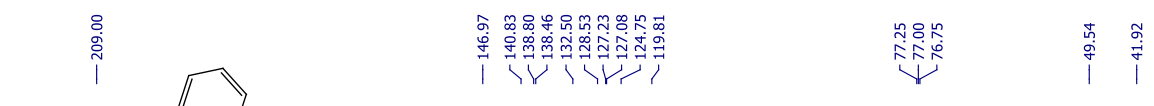

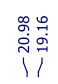
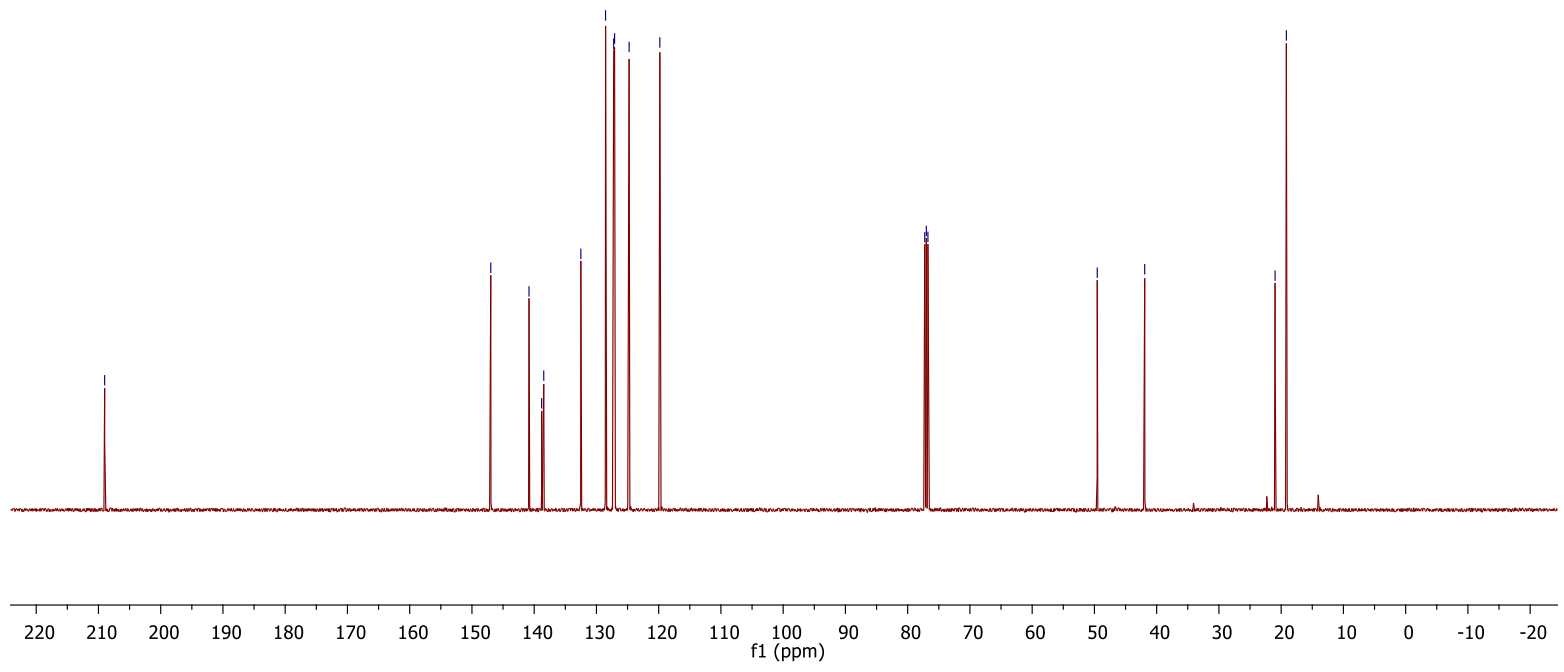

Figure S35: ${ }^{13} \mathrm{C}-\mathrm{NMR}(125 \mathrm{MHz})$ in $\mathrm{CDCl}_{3}$ of $1 \mathrm{n}$

S-40 


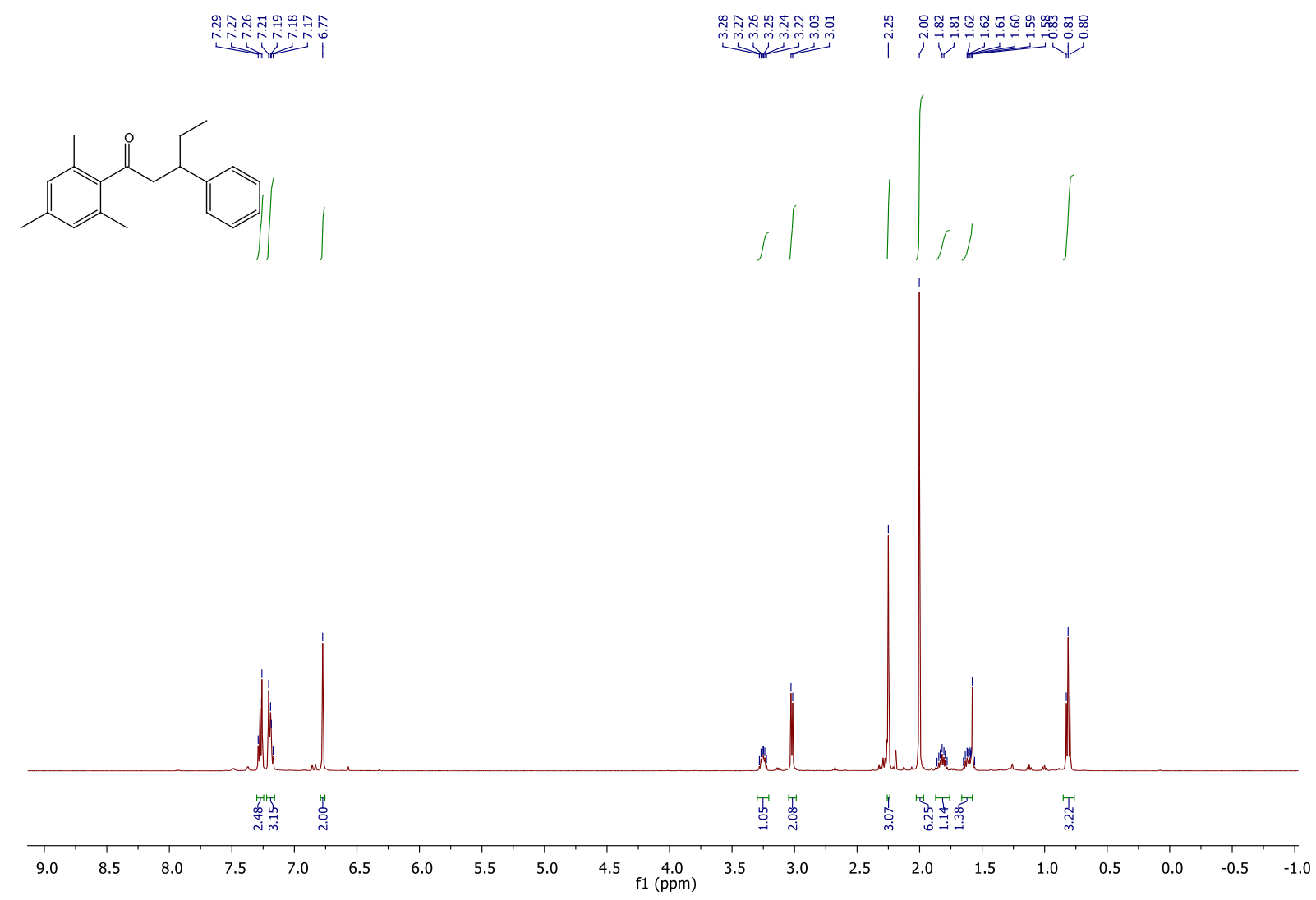

Figure S36: ${ }^{1} \mathrm{H}-\mathrm{NMR}(500 \mathrm{MHz})$ in $\mathrm{CDCl}_{3}$ of 10

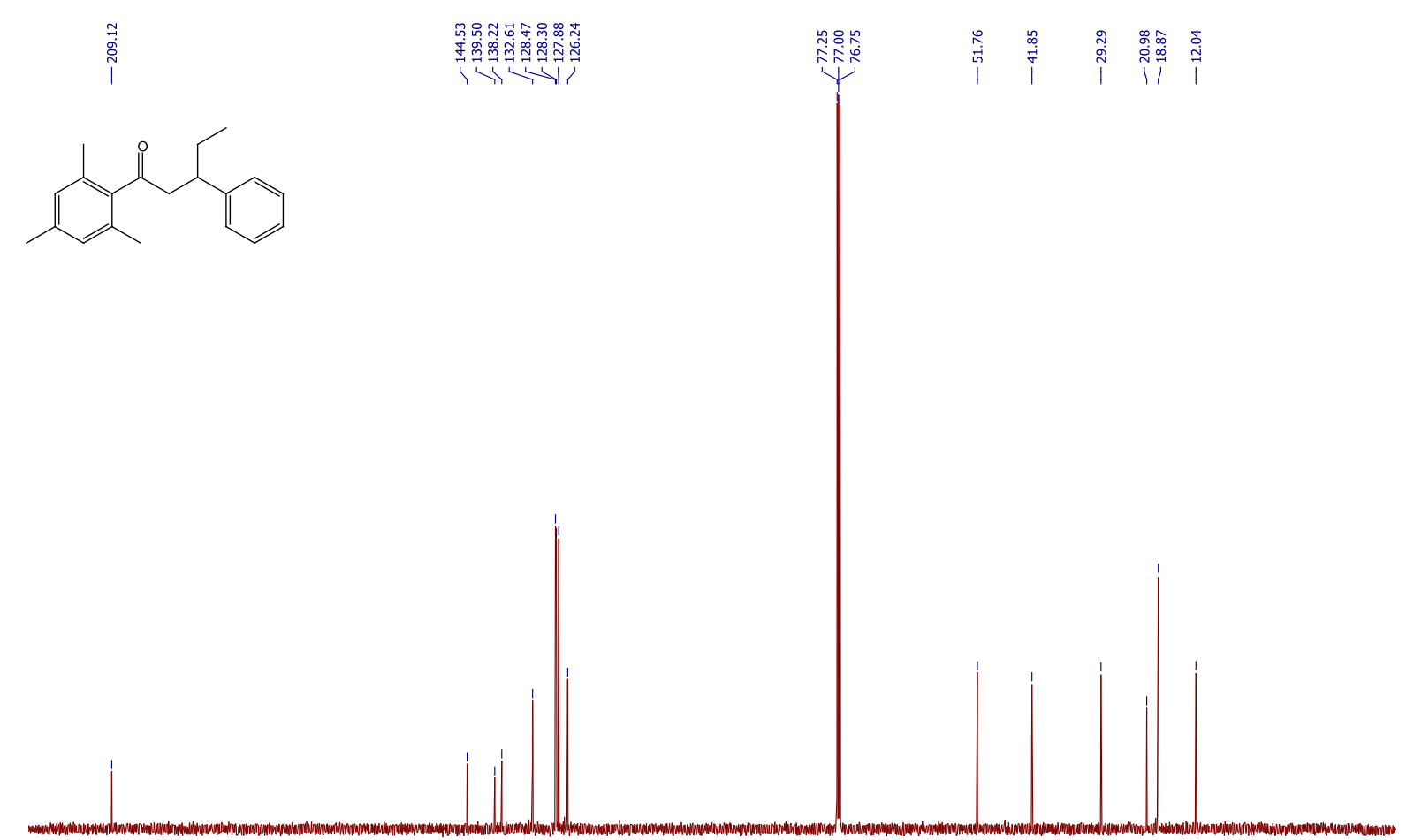

$\begin{array}{lllllllllllllllllllllllllllllllllll}220 & 210 & 200 & 190 & 180 & 170 & 160 & 150 & 140 & 130 & 120 & 110 & 100 & 90 & 80 & 70 & 60 & 50 & 40 & 30 & 20 & 10 & 0 & -10 & -20\end{array}$

Figure S37: ${ }^{13} \mathrm{C}-\mathrm{NMR}(125 \mathrm{MHz})$ in $\mathrm{CDCl}_{3}$ of 10 

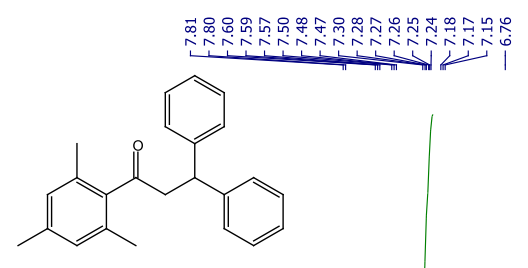

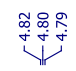

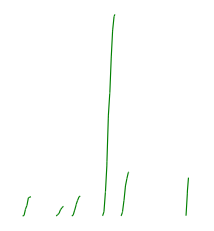

ํํํ

$\stackrel{\stackrel{\sim}{\sim}}{i} \stackrel{\infty}{i}$

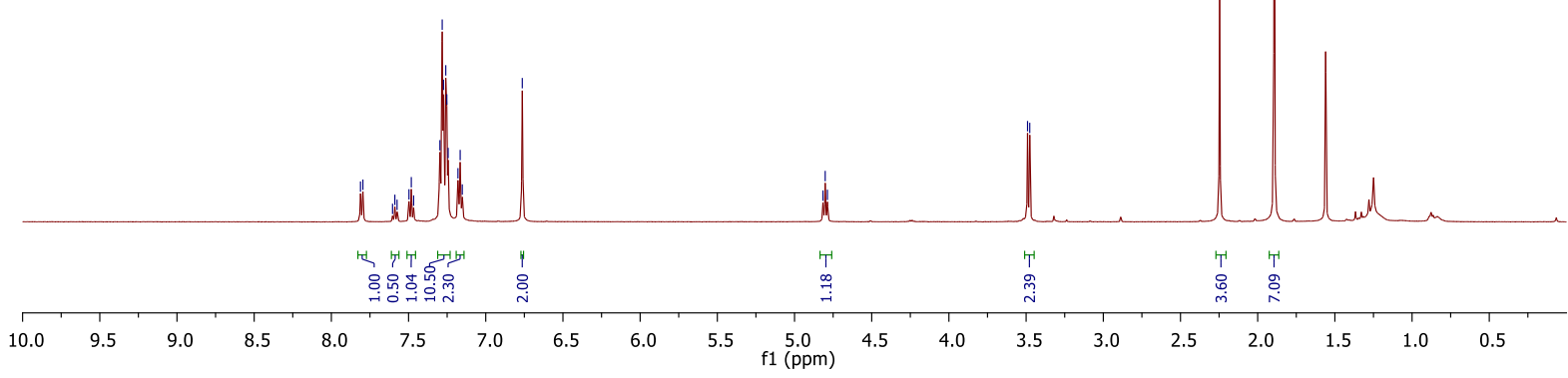

Figure S38: ${ }^{1} \mathrm{H}-\mathrm{NMR}(500 \mathrm{MHz})$ in $\mathrm{CDCl}_{3}$ of $1 \mathrm{p}$
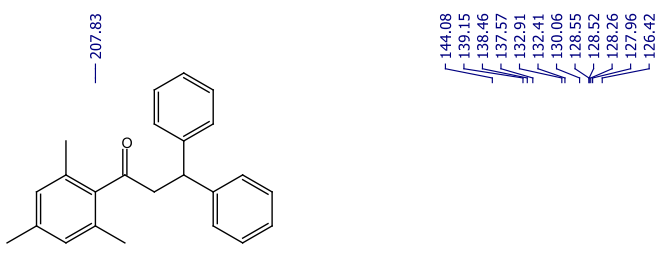

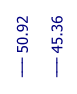

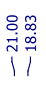
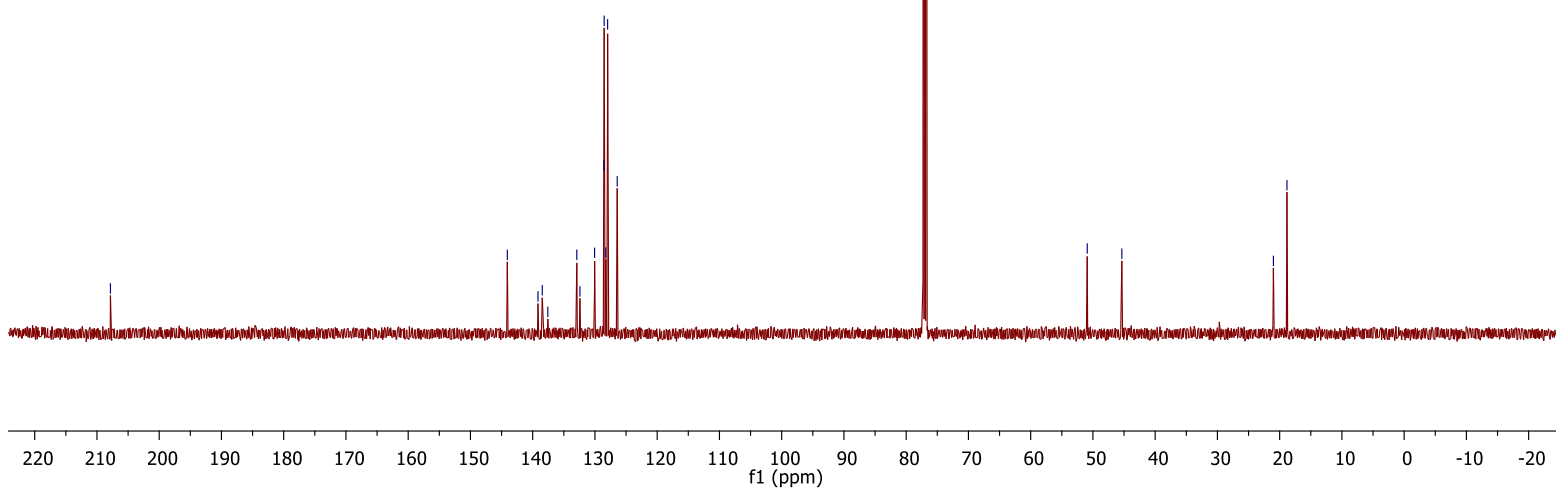

Figure S39: ${ }^{13} \mathrm{C}-\mathrm{NMR}(125 \mathrm{MHz})$ in $\mathrm{CDCl}_{3}$ of $1 \mathrm{p}$

S-42 


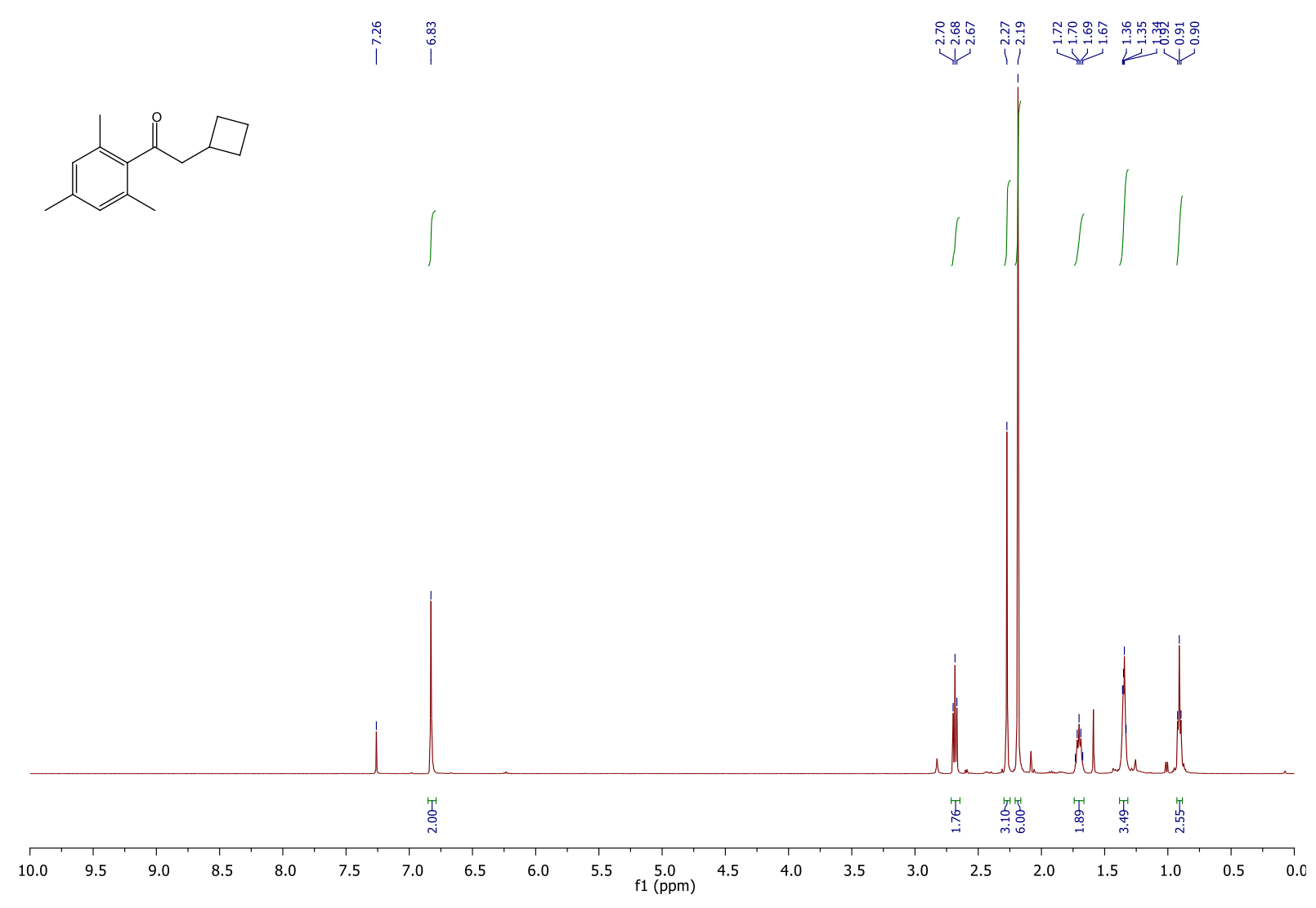

Figure S40: ${ }^{1} \mathrm{H}-\mathrm{NMR}(500 \mathrm{MHz})$ in $\mathrm{CDCl}_{3}$ of $1 \mathrm{q}$
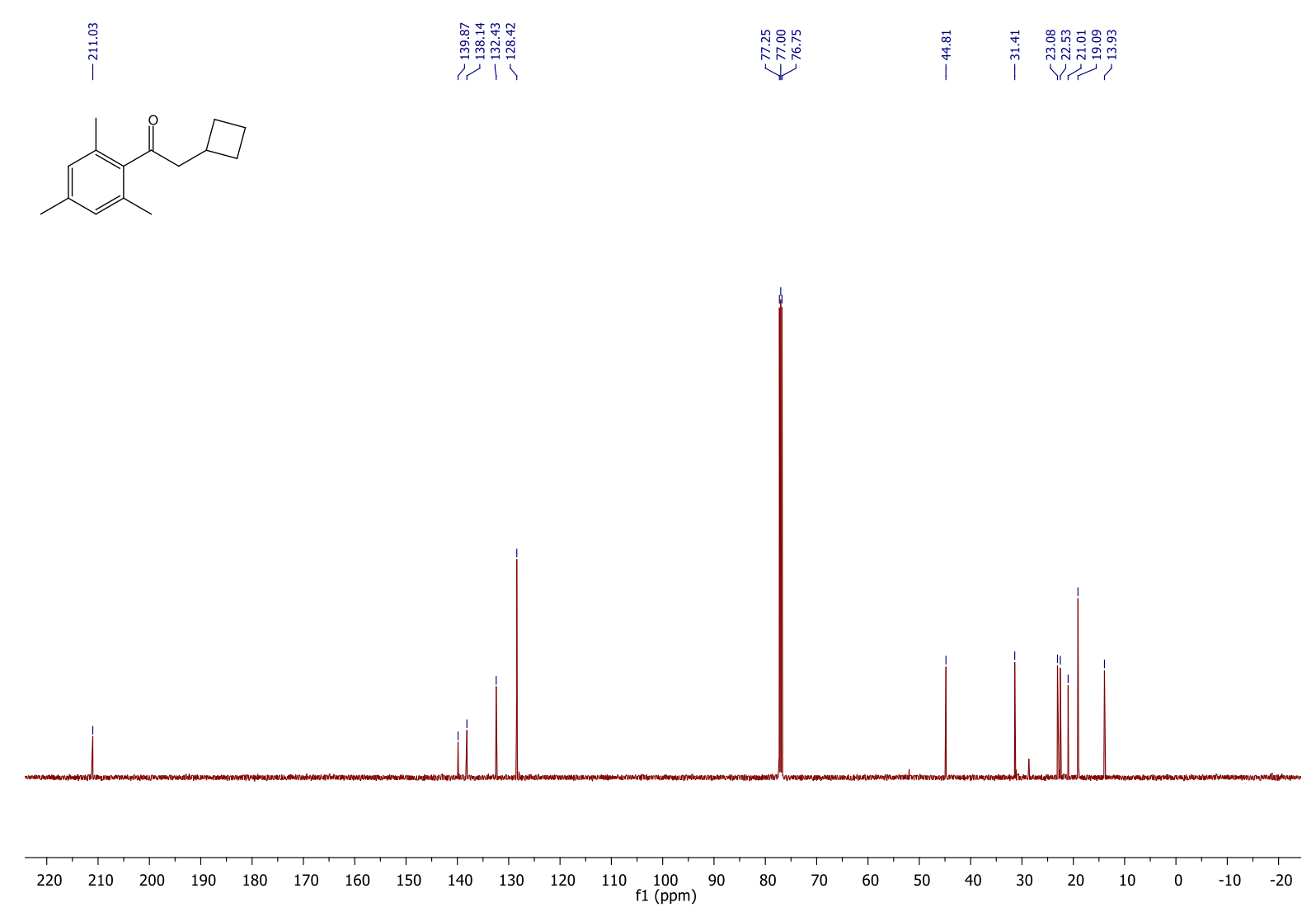

Figure S41: ${ }^{13} \mathrm{C}-\mathrm{NMR}(125 \mathrm{MHz})$ in $\mathrm{CDCl}_{3}$ of $1 \mathrm{q}$ 


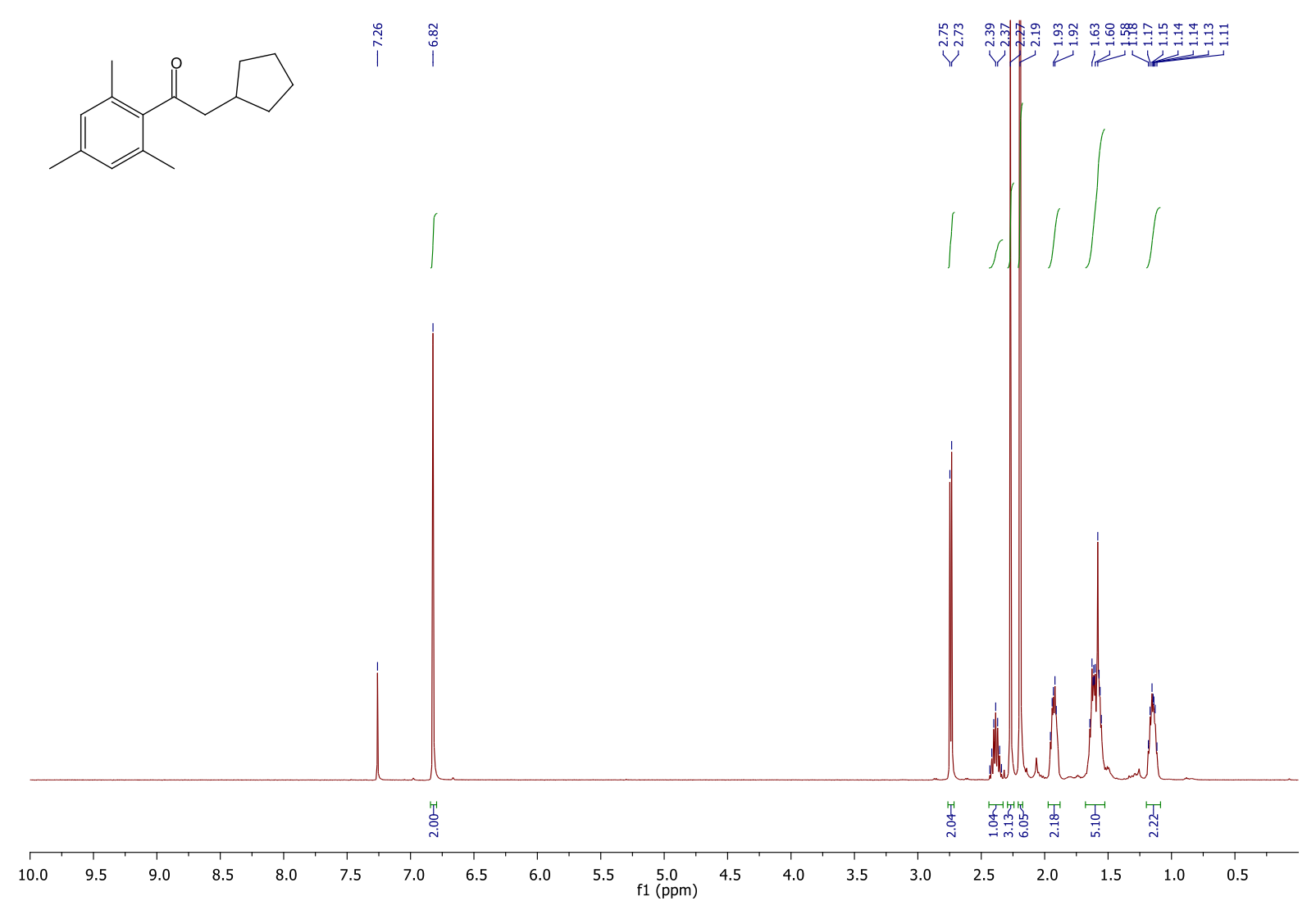

Figure S42: ${ }^{1} \mathrm{H}-\mathrm{NMR}(500 \mathrm{MHz})$ in $\mathrm{CDCl}_{3}$ of $1 \mathrm{r}$

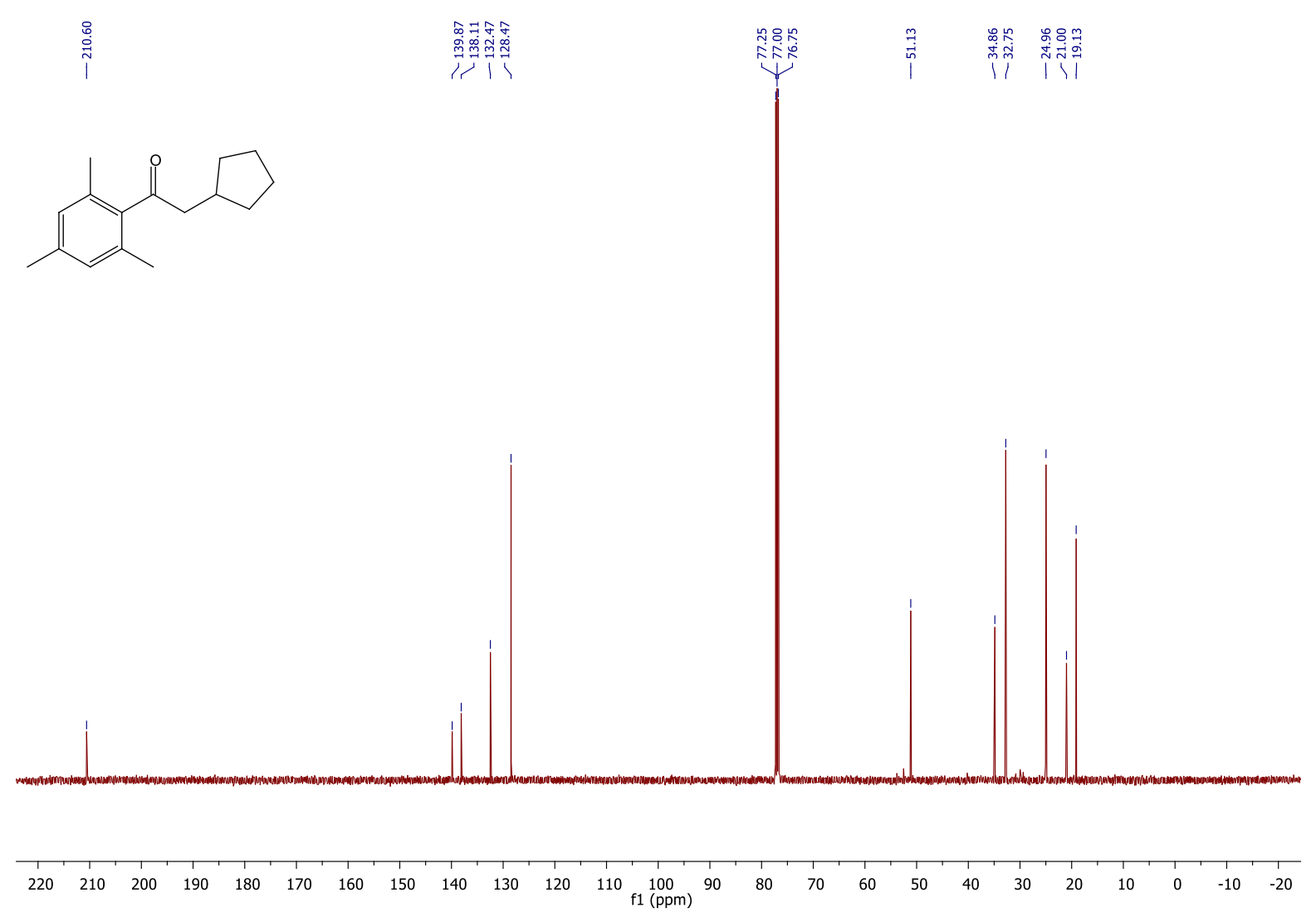

Figure S43: ${ }^{13} \mathrm{C}-\mathrm{NMR}(125 \mathrm{MHz})$ in $\mathrm{CDCl}_{3}$ of $1 \mathrm{r}$ 


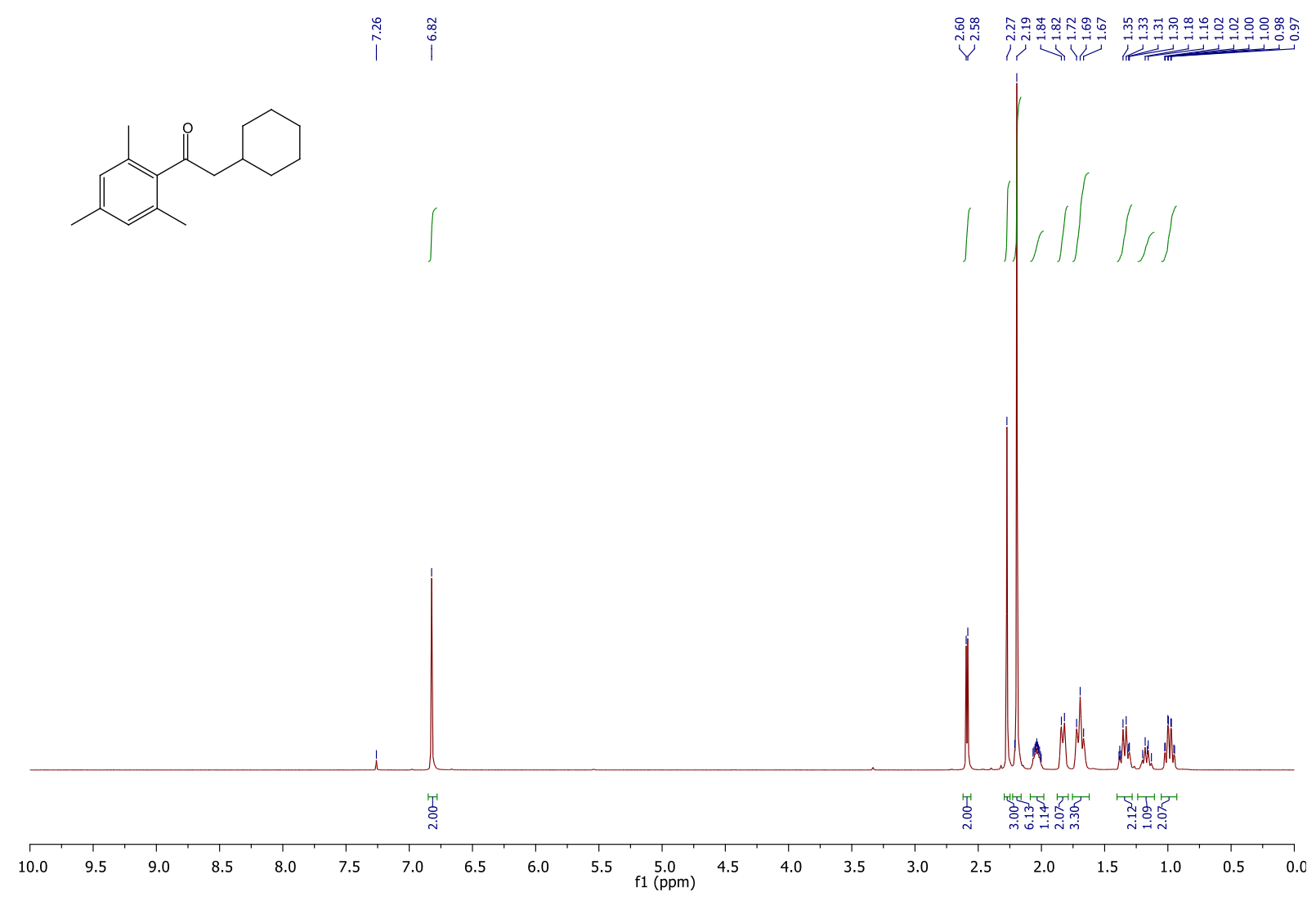

Figure S44: ${ }^{1} \mathrm{H}-\mathrm{NMR}(500 \mathrm{MHz})$ in $\mathrm{CDCl}_{3}$ of $1 \mathrm{~s}$

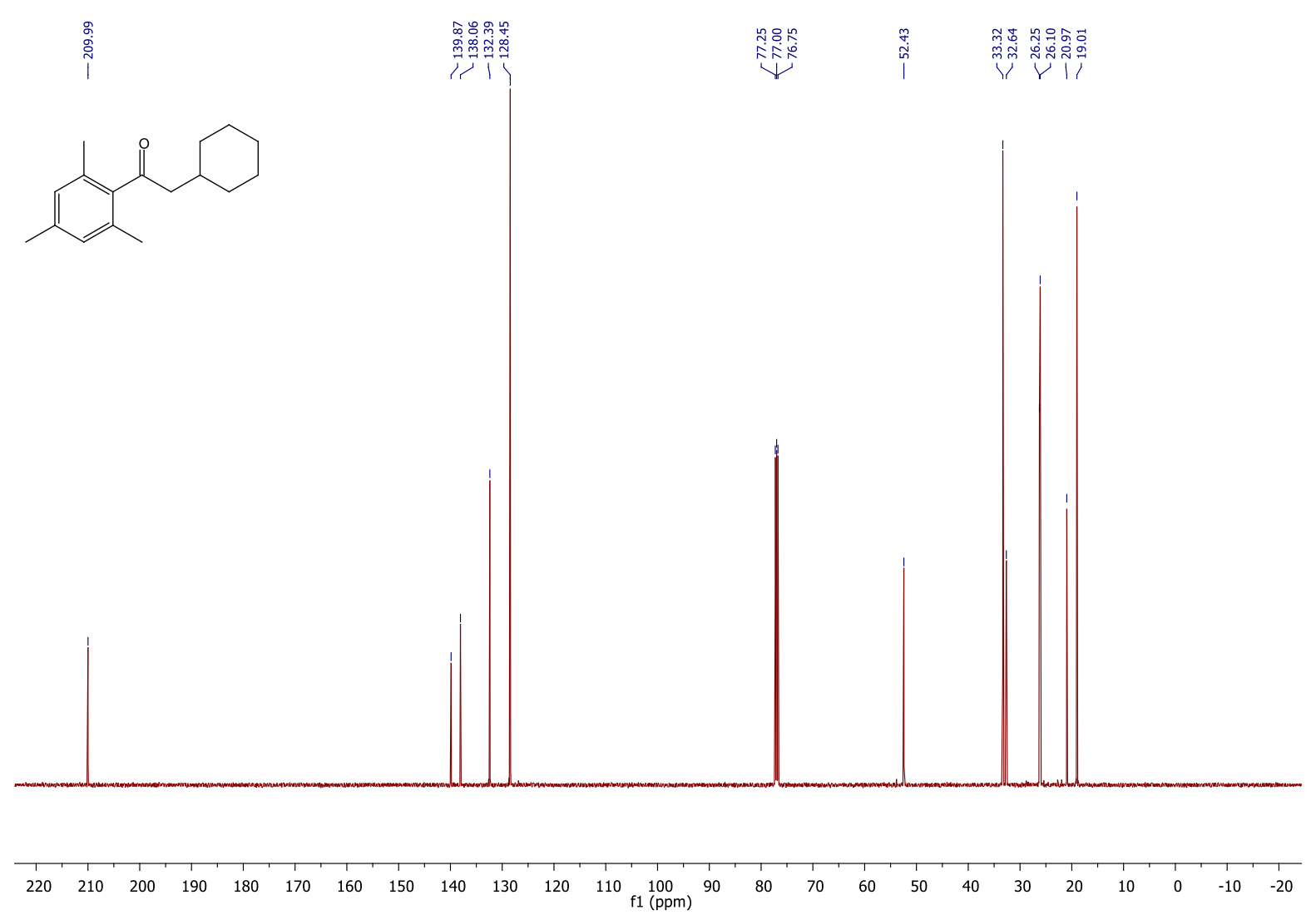

Figure S45: ${ }^{13} \mathrm{C}-\mathrm{NMR}(125 \mathrm{MHz})$ in $\mathrm{CDCl}_{3}$ of $1 \mathrm{~s}$ 


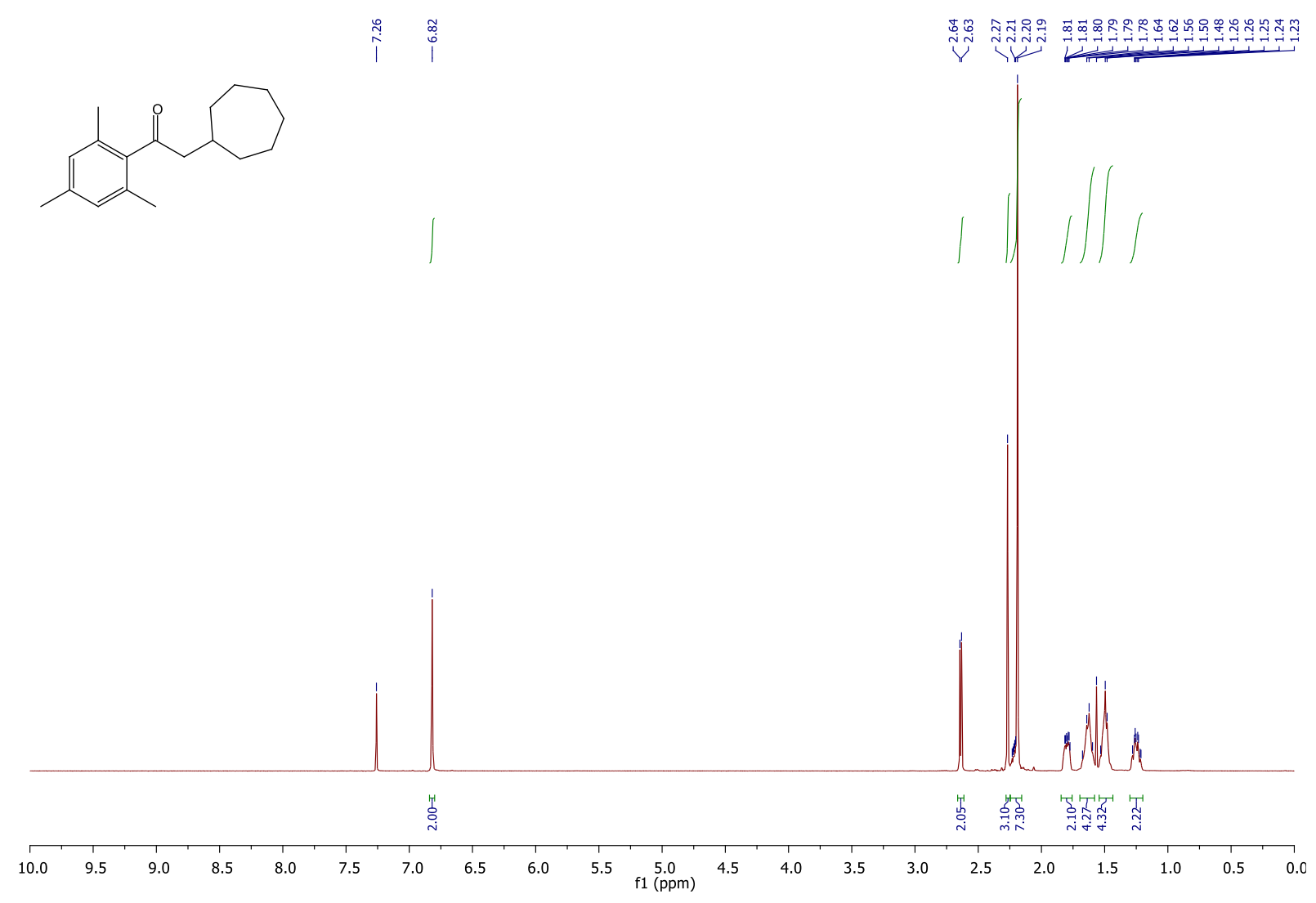

Figure S46: ${ }^{1} \mathrm{H}-\mathrm{NMR}(500 \mathrm{MHz})$ in $\mathrm{CDCl}_{3}$ of $1 \mathrm{t}$

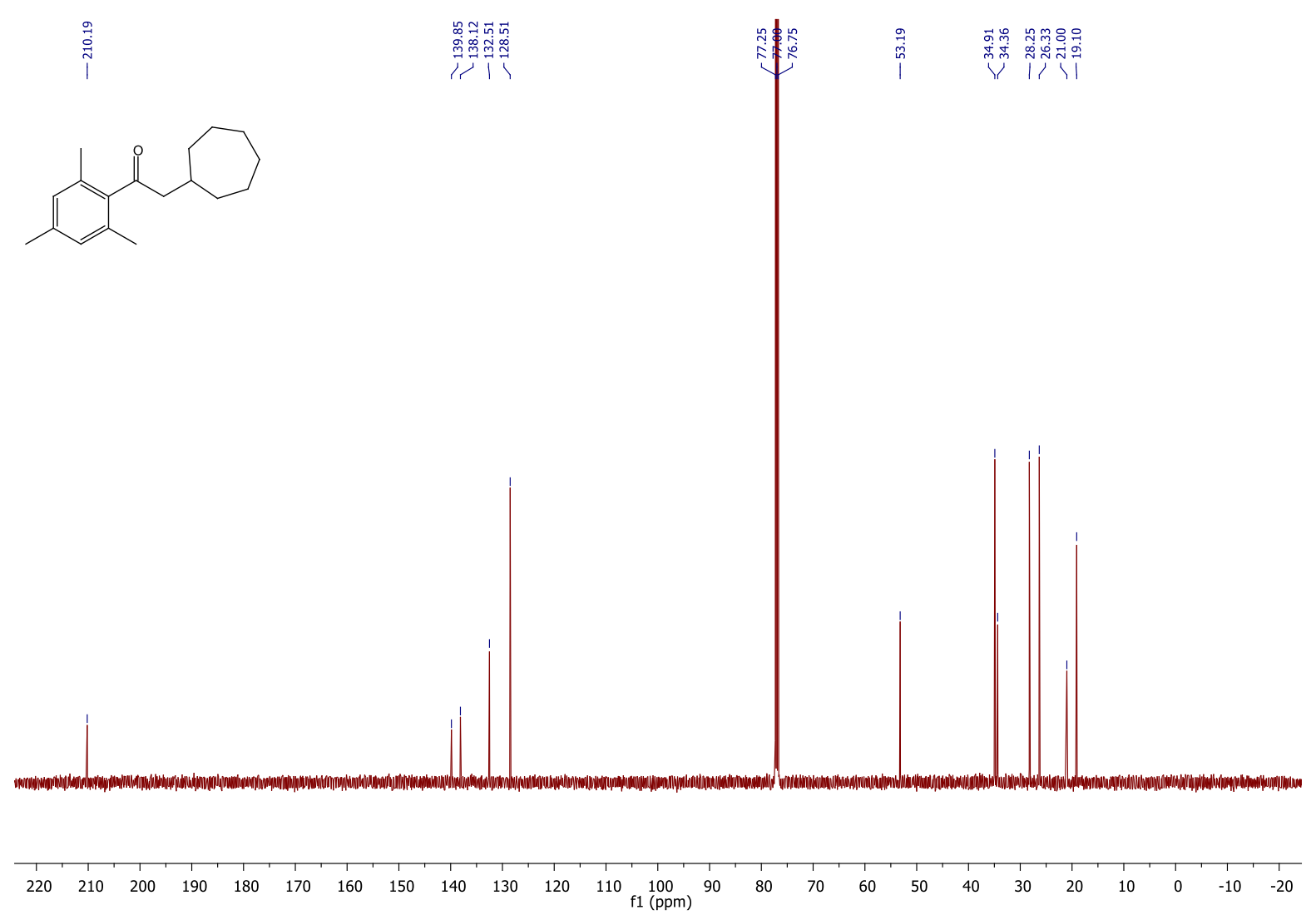

Figure S47: ${ }^{13} \mathrm{C}$-NMR (125 MHz) in $\mathrm{CDCl}_{3}$ of $1 \mathrm{t}$ 


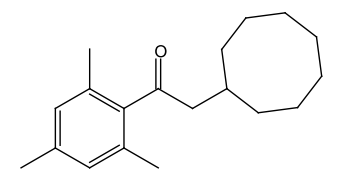

$\checkmark$ ข

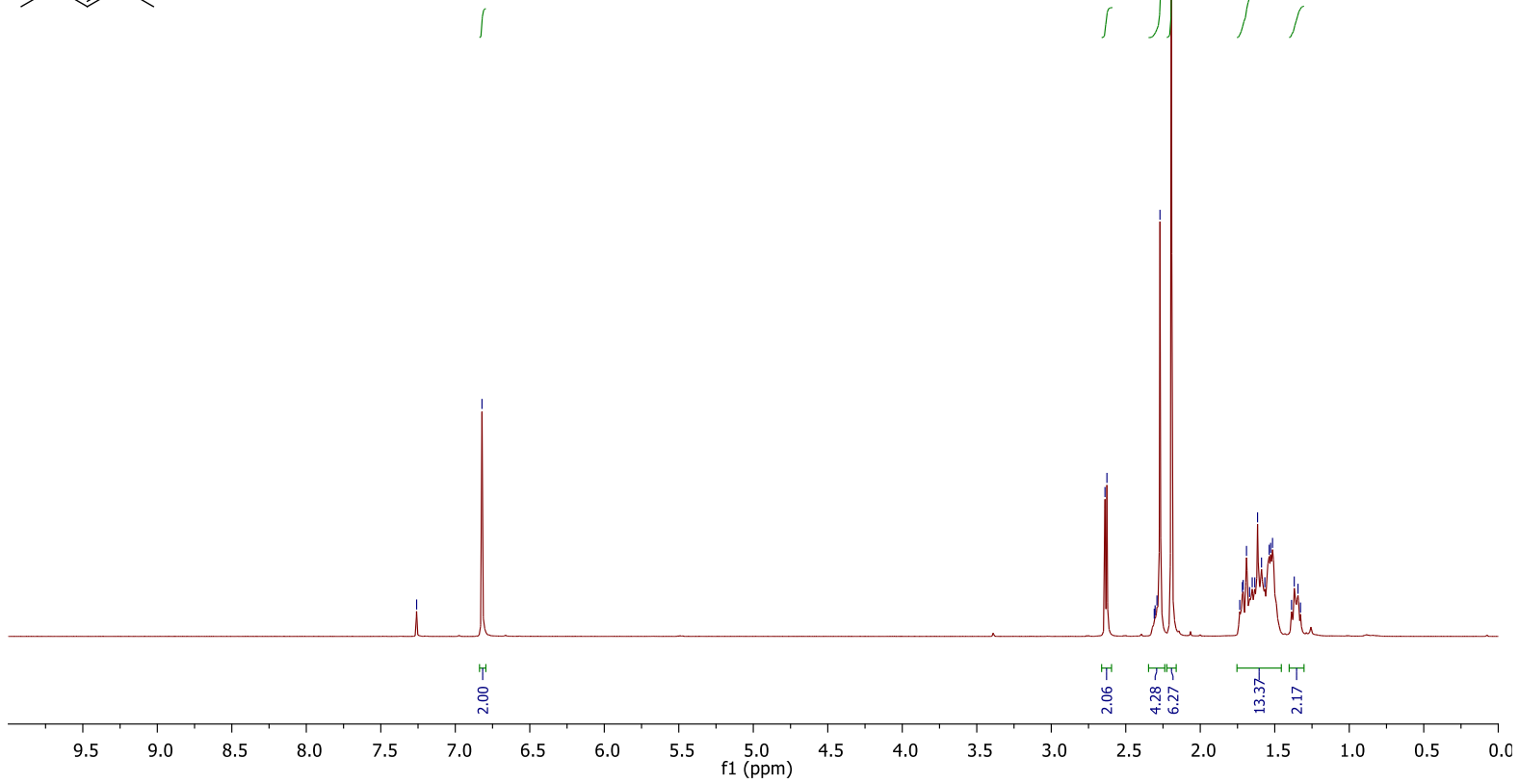

Figure S48: ${ }^{1} \mathrm{H}-\mathrm{NMR}(500 \mathrm{MHz})$ in $\mathrm{CDCl}_{3}$ of $1 \mathrm{u}$

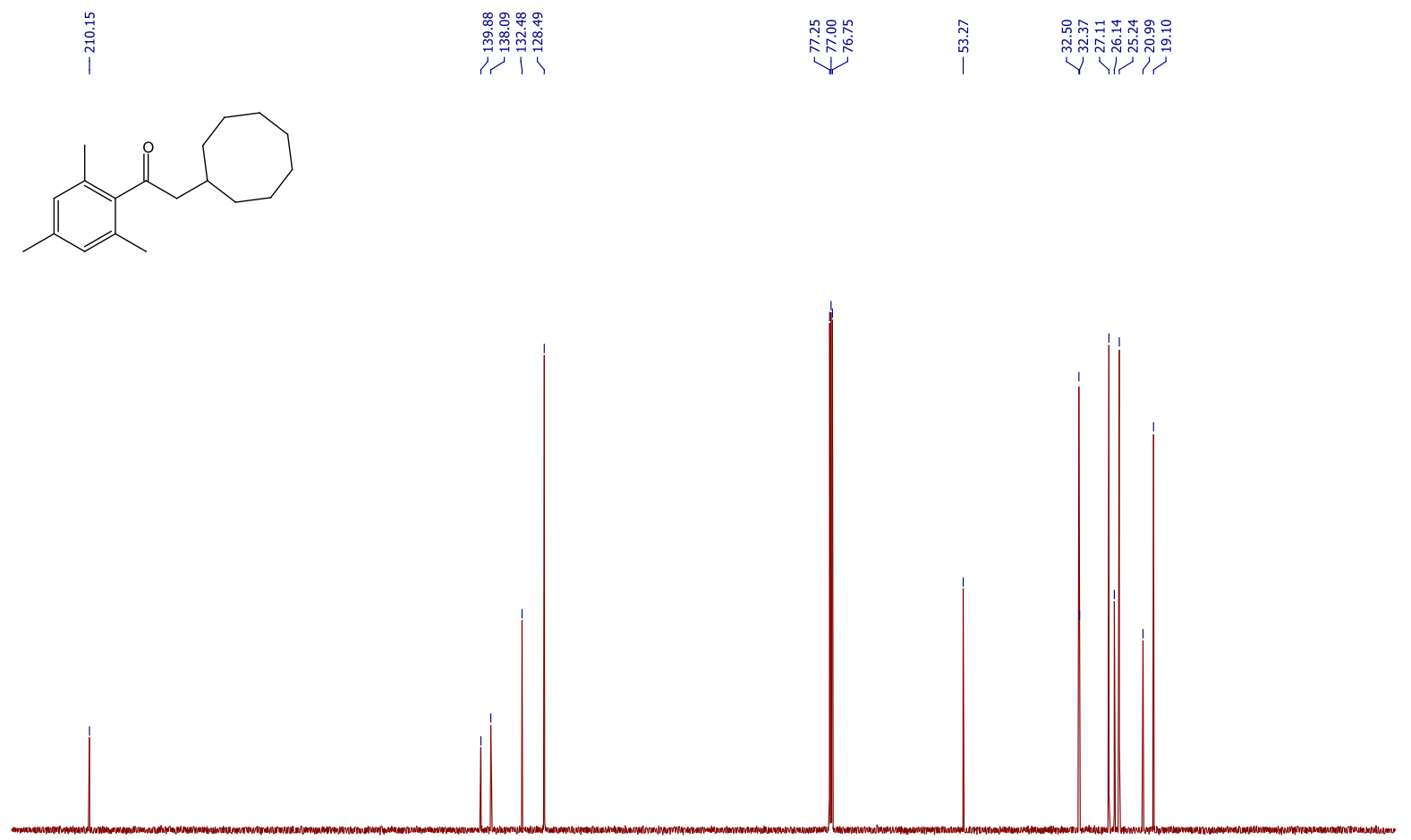

$\begin{array}{lllllllllllllllllllllllllllllll}220 & 210 & 200 & 190 & 180 & 170 & 160 & 150 & 140 & 130 & 120 & 110 & 100 & 90 & 80 & 70 & 60 & 50 & 40 & 30 & 20 & 10 & 0 & -10 & -20\end{array}$

Figure S49: ${ }^{13} \mathrm{C}-\mathrm{NMR}(125 \mathrm{MHz})$ in $\mathrm{CDCl}_{3}$ of $1 \mathrm{u}$ 


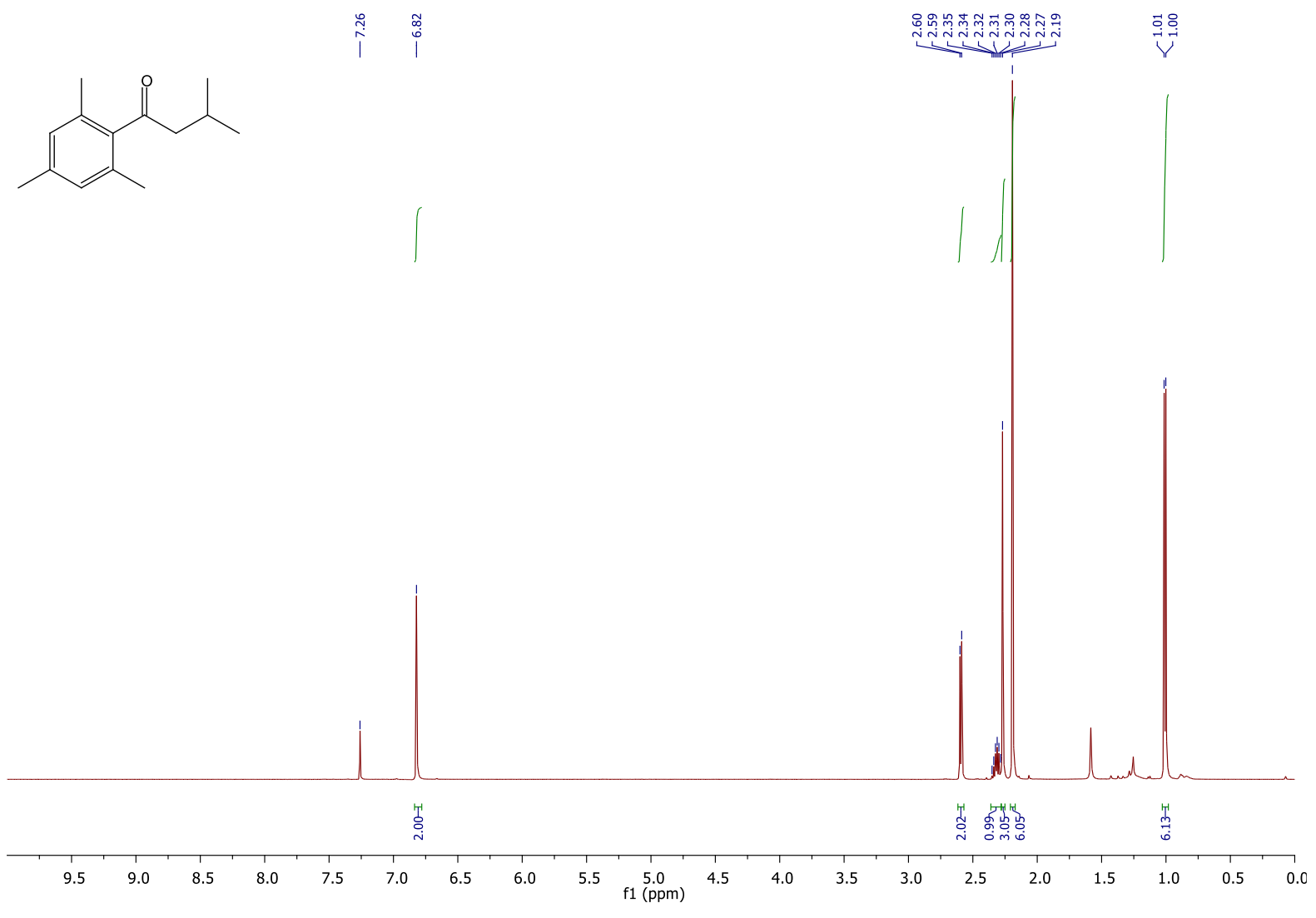

Figure S50: ${ }^{1} \mathrm{H}-\mathrm{NMR}(500 \mathrm{MHz})$ in $\mathrm{CDCl}_{3}$ of $1 \mathrm{v}$

$\underset{\substack{n \\ \stackrel{n}{1}}}{\stackrel{1}{1}}$

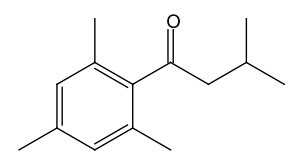

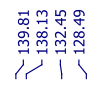

串

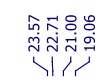

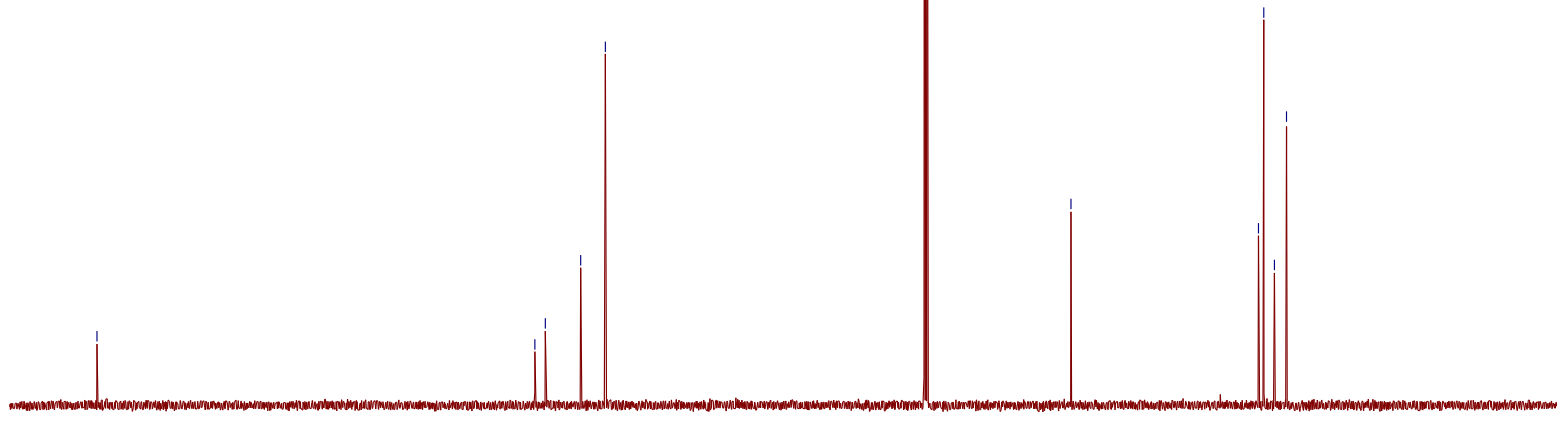

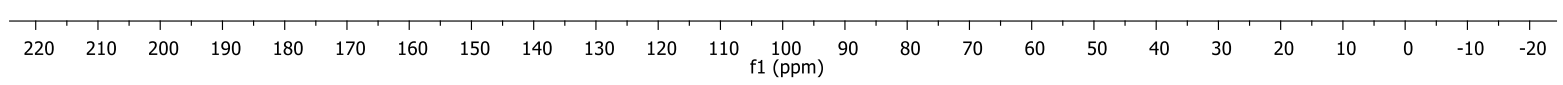

Figure S51: ${ }^{13} \mathrm{C}-\mathrm{NMR}(125 \mathrm{MHz})$ in $\mathrm{CDCl}_{3}$ of $1 \mathrm{v}$

S-48 


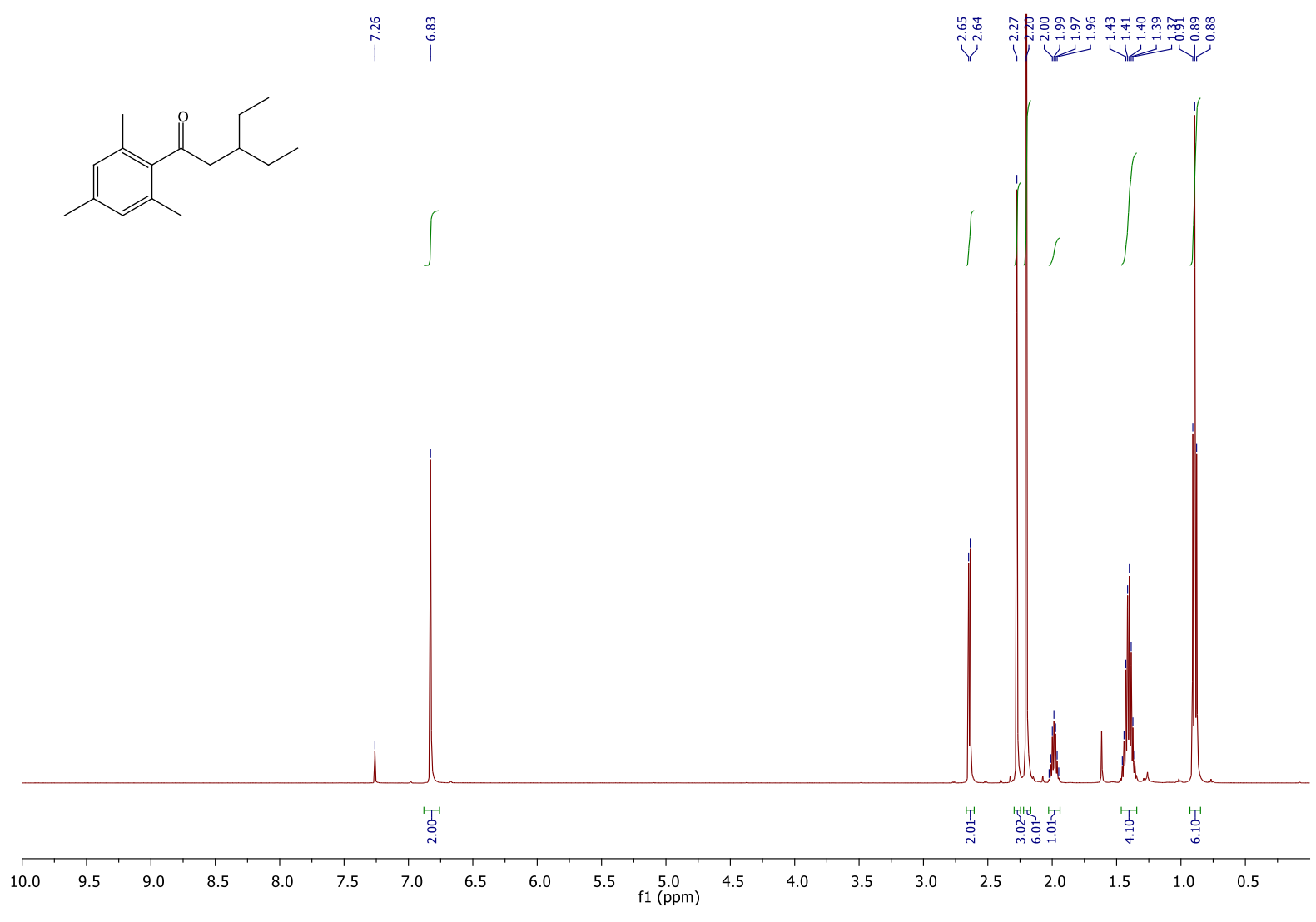

Figure S52: ${ }^{1} \mathrm{H}-\mathrm{NMR}(500 \mathrm{MHz})$ in $\mathrm{CDCl}_{3}$ of $1 \mathrm{w}$

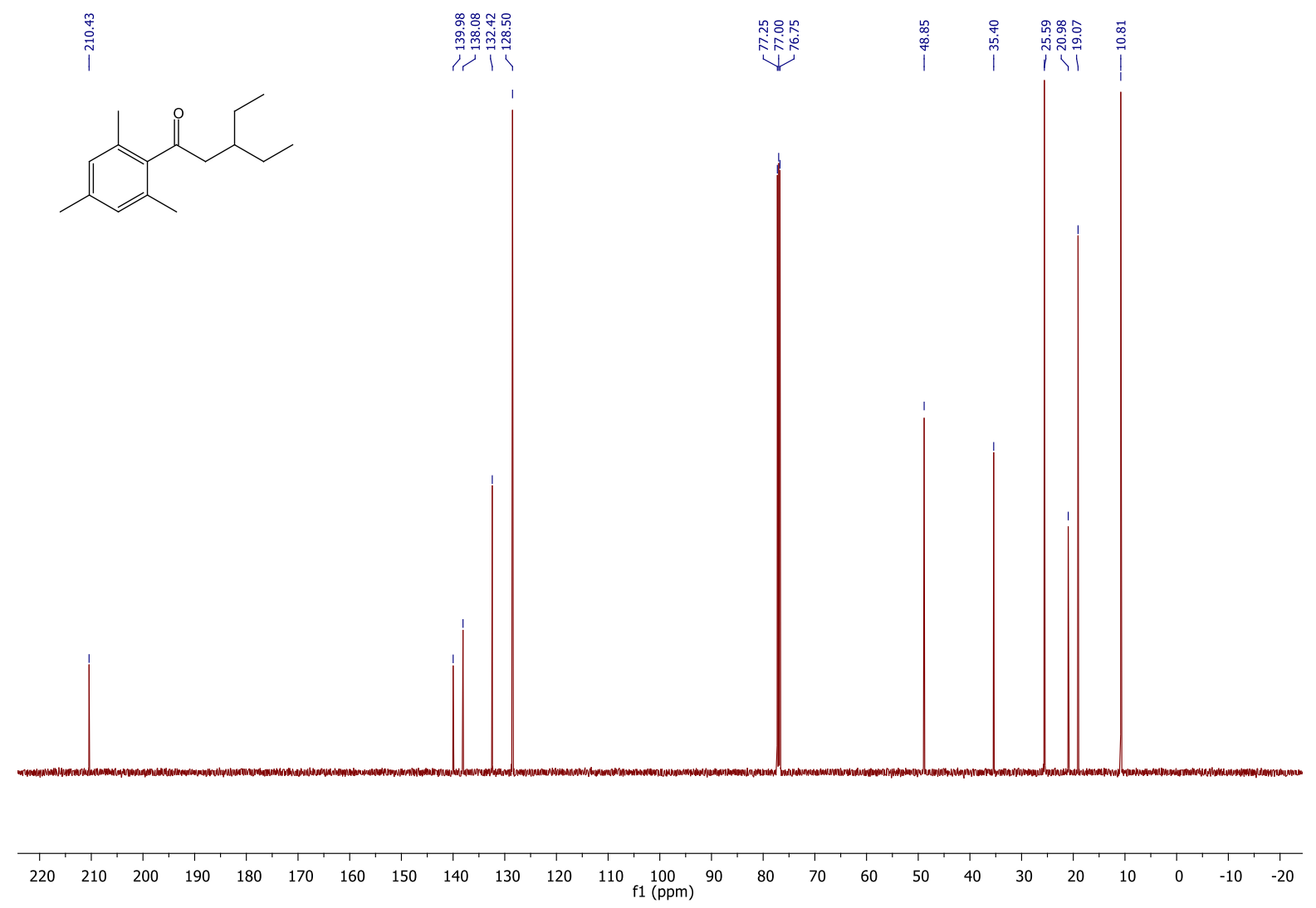

Figure S53: ${ }^{13} \mathrm{C}-\mathrm{NMR}(125 \mathrm{MHz})$ in $\mathrm{CDCl}_{3}$ of $1 \mathrm{w}$

S-49 


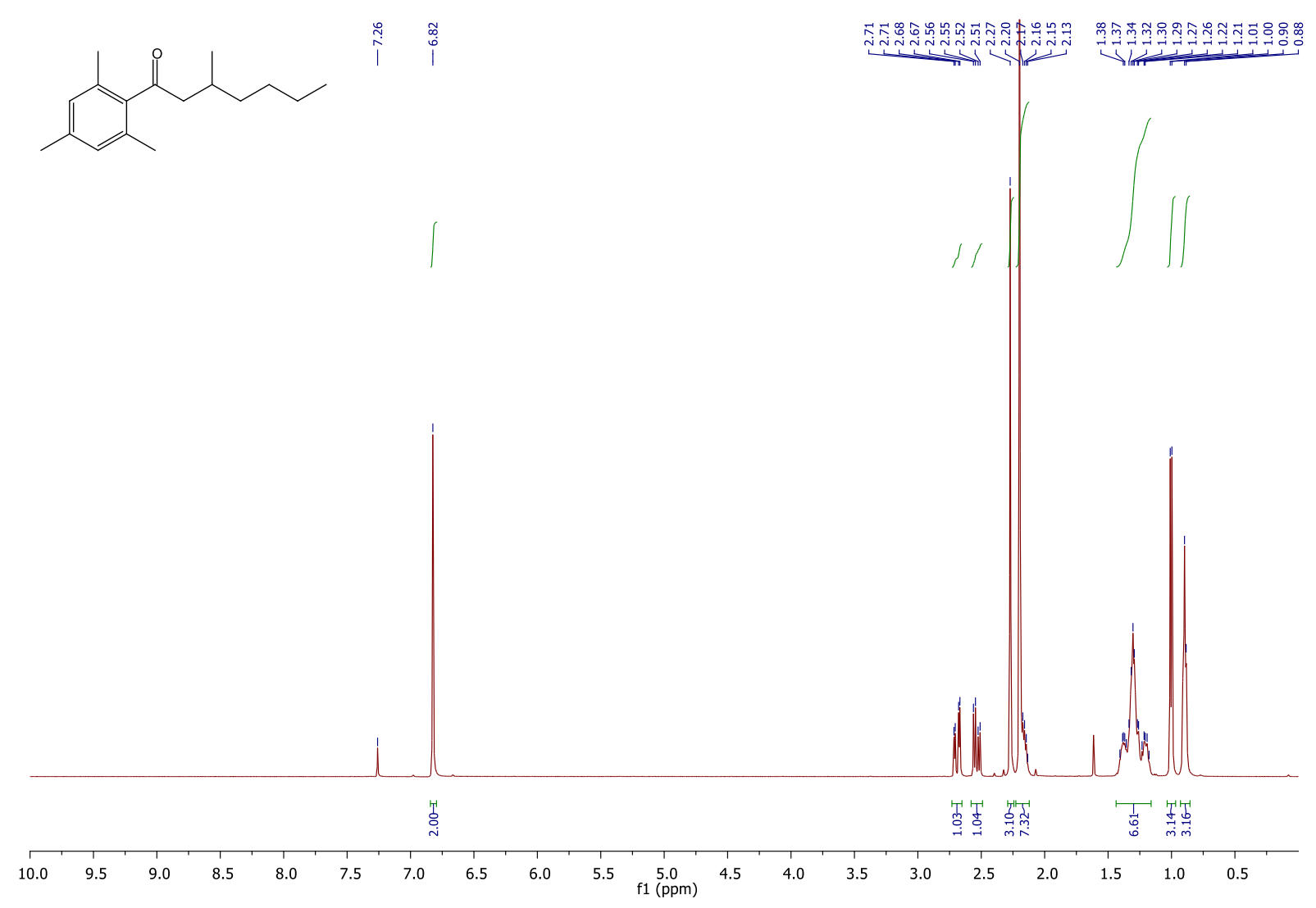

Figure S54: ${ }^{1} \mathrm{H}-\mathrm{NMR}(500 \mathrm{MHz})$ in $\mathrm{CDCl}_{3}$ of $1 \mathrm{x}$
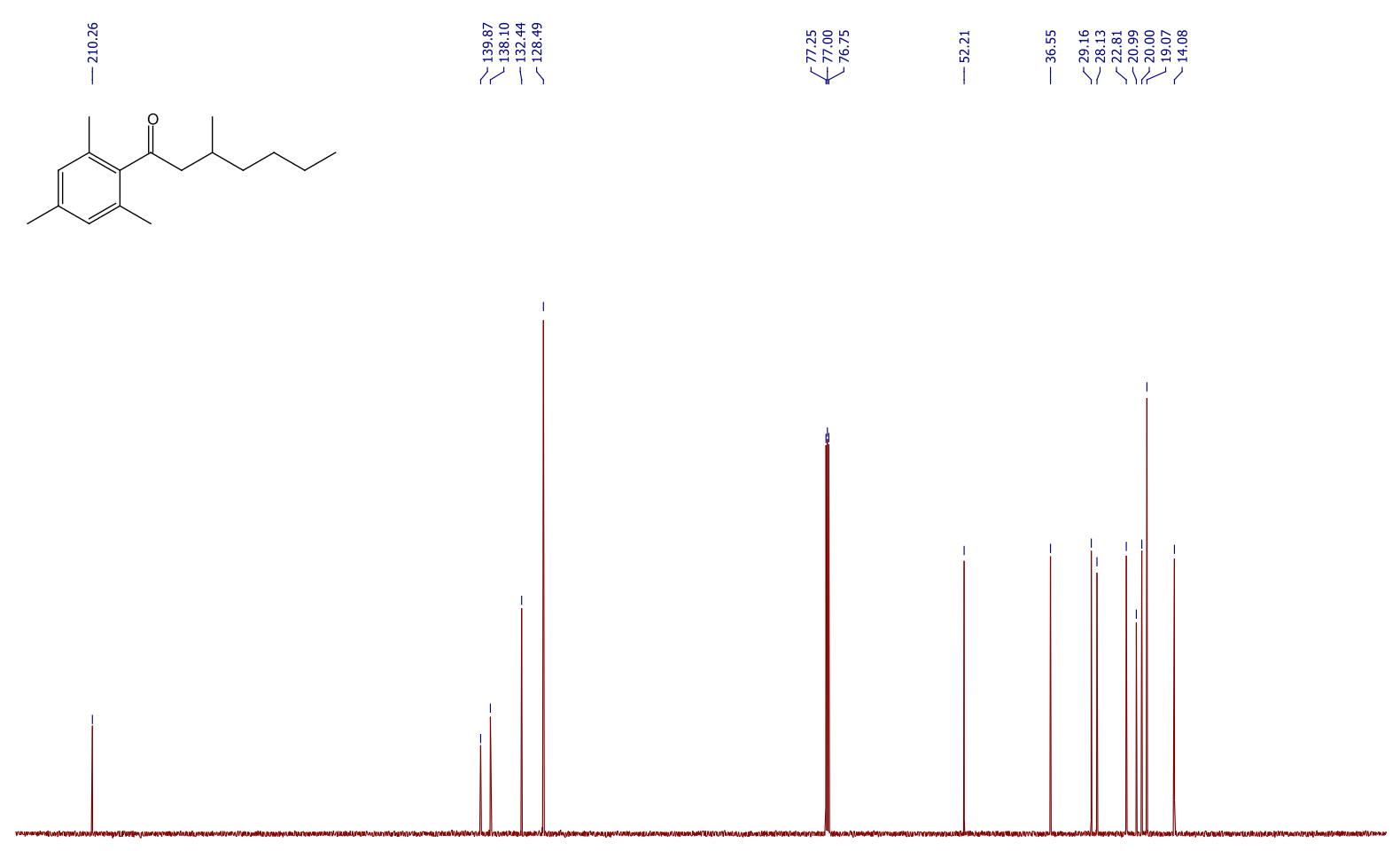

$\begin{array}{lllllllllllllllllllllllllllllll}220 & 210 & 200 & 190 & 180 & 170 & 160 & 150 & 140 & 130 & 120 & 110 & 100 & 90 & 80 & 70 & 60 & 50 & 40 & 30 & 20 & 10 & 0 & -10 & -20\end{array}$

Figure S55: ${ }^{13} \mathrm{C}-\mathrm{NMR}(125 \mathrm{MHz})$ in $\mathrm{CDCl}_{3}$ of $1 \mathrm{x}$ 


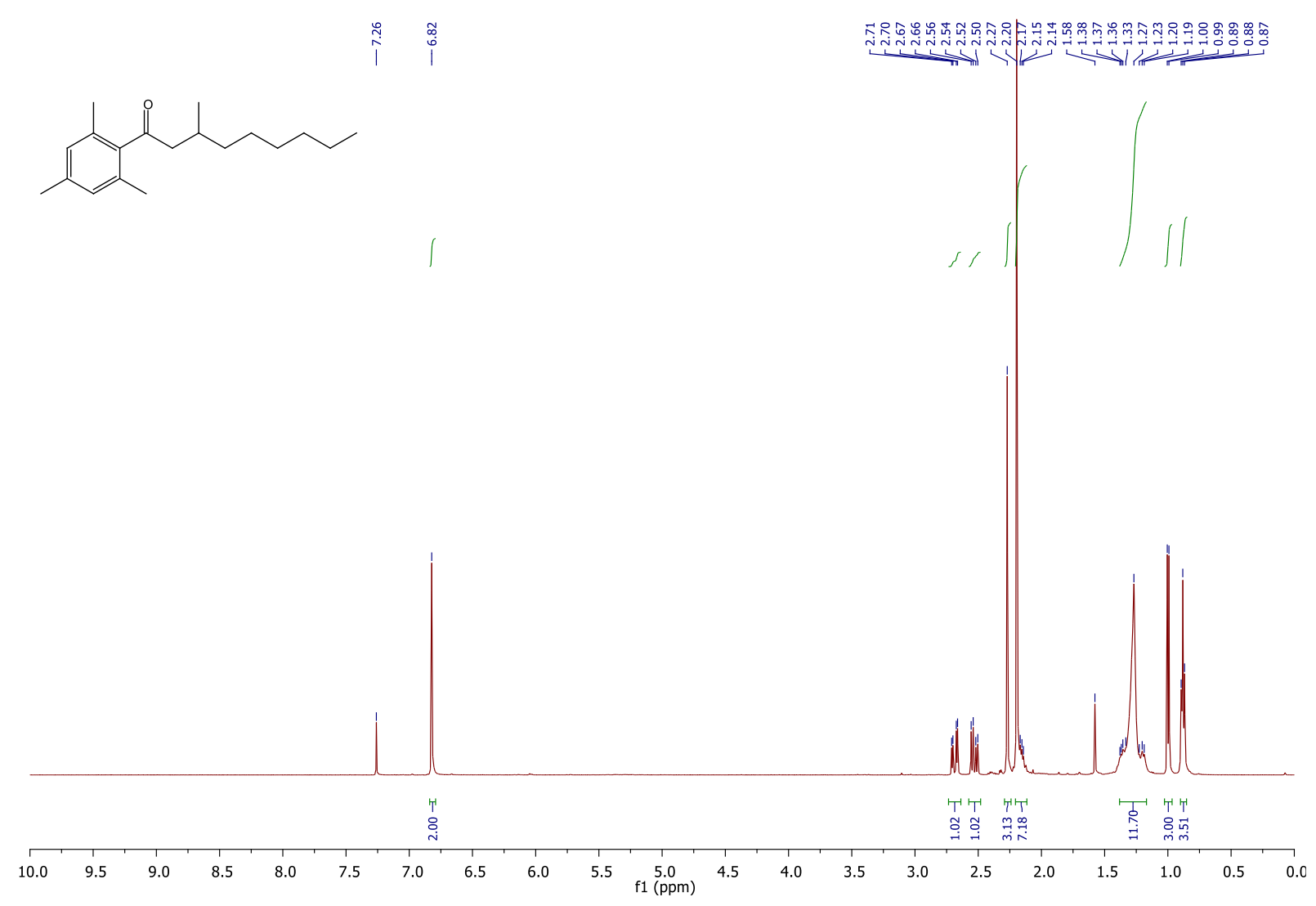

Figure S56: ${ }^{1} \mathrm{H}-\mathrm{NMR}(500 \mathrm{MHz})$ in $\mathrm{CDCl}_{3}$ of $1 \mathrm{y}$

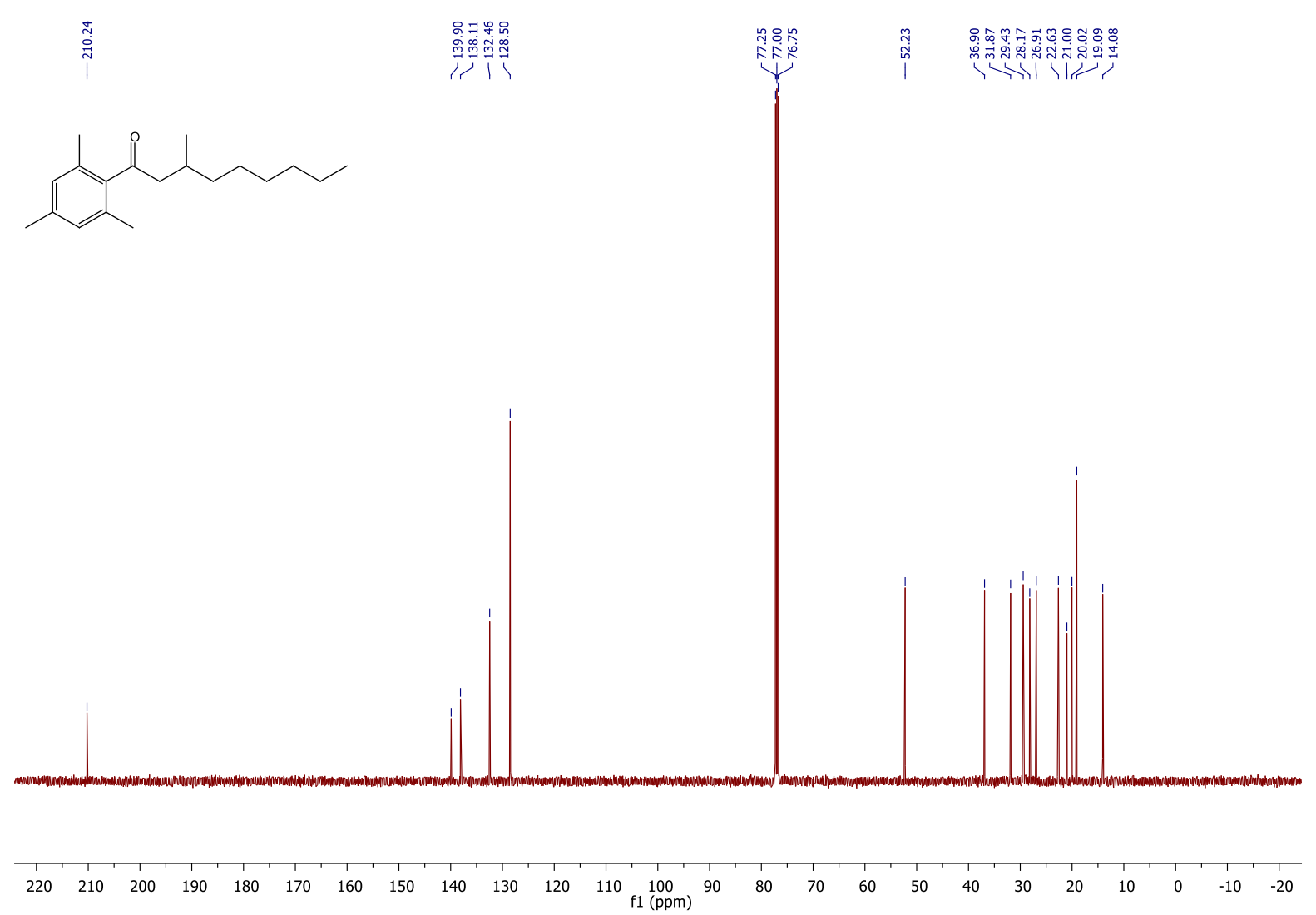

Figure S57: ${ }^{13} \mathrm{C}-\mathrm{NMR}\left(125 \mathrm{MHz}\right.$ ) in $\mathrm{CDCl}_{3}$ of $1 \mathrm{y}$ 


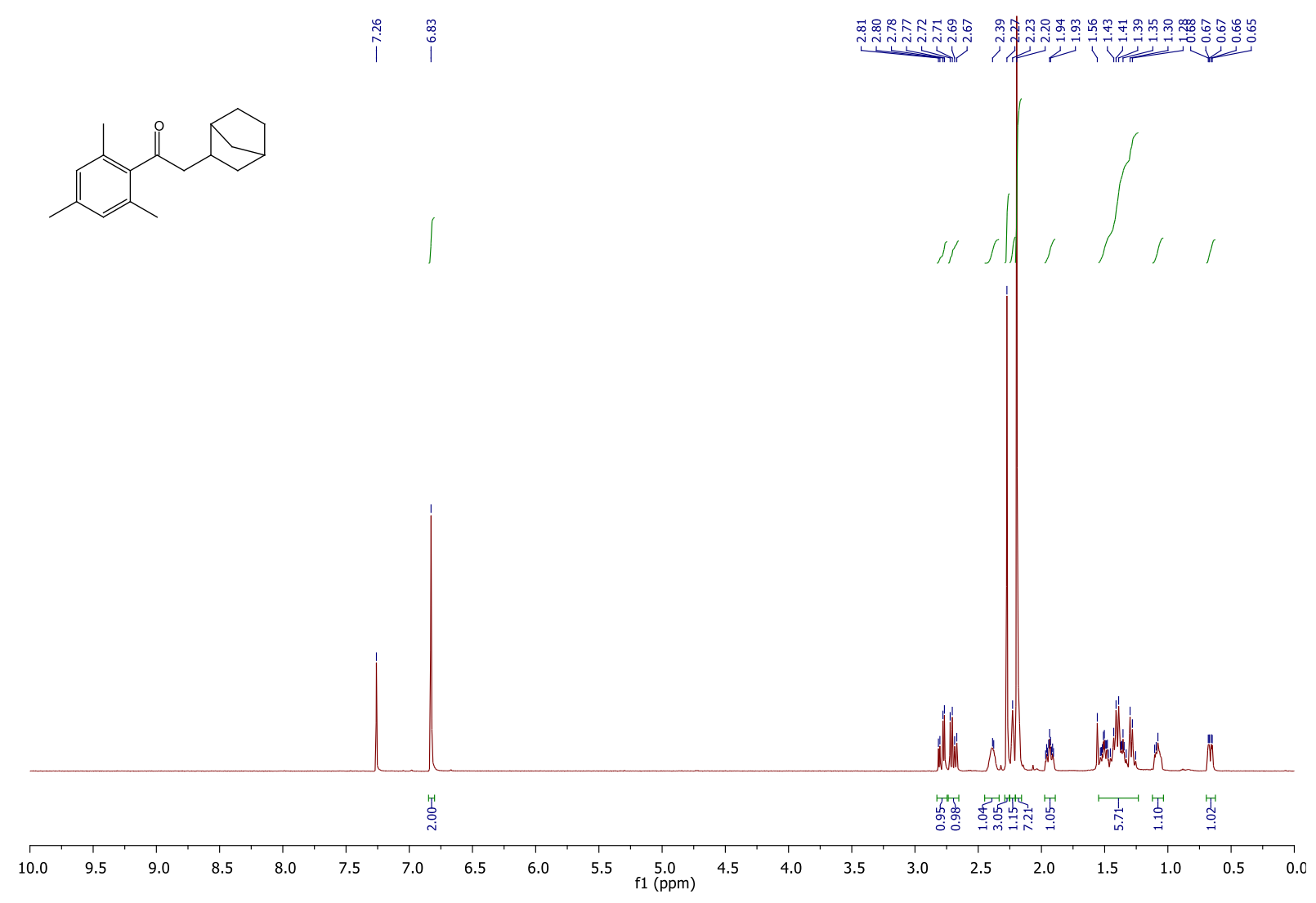

Figure S58: ${ }^{1} \mathrm{H}-\mathrm{NMR}(500 \mathrm{MHz})$ in $\mathrm{CDCl}_{3}$ of $1 \mathrm{z}$

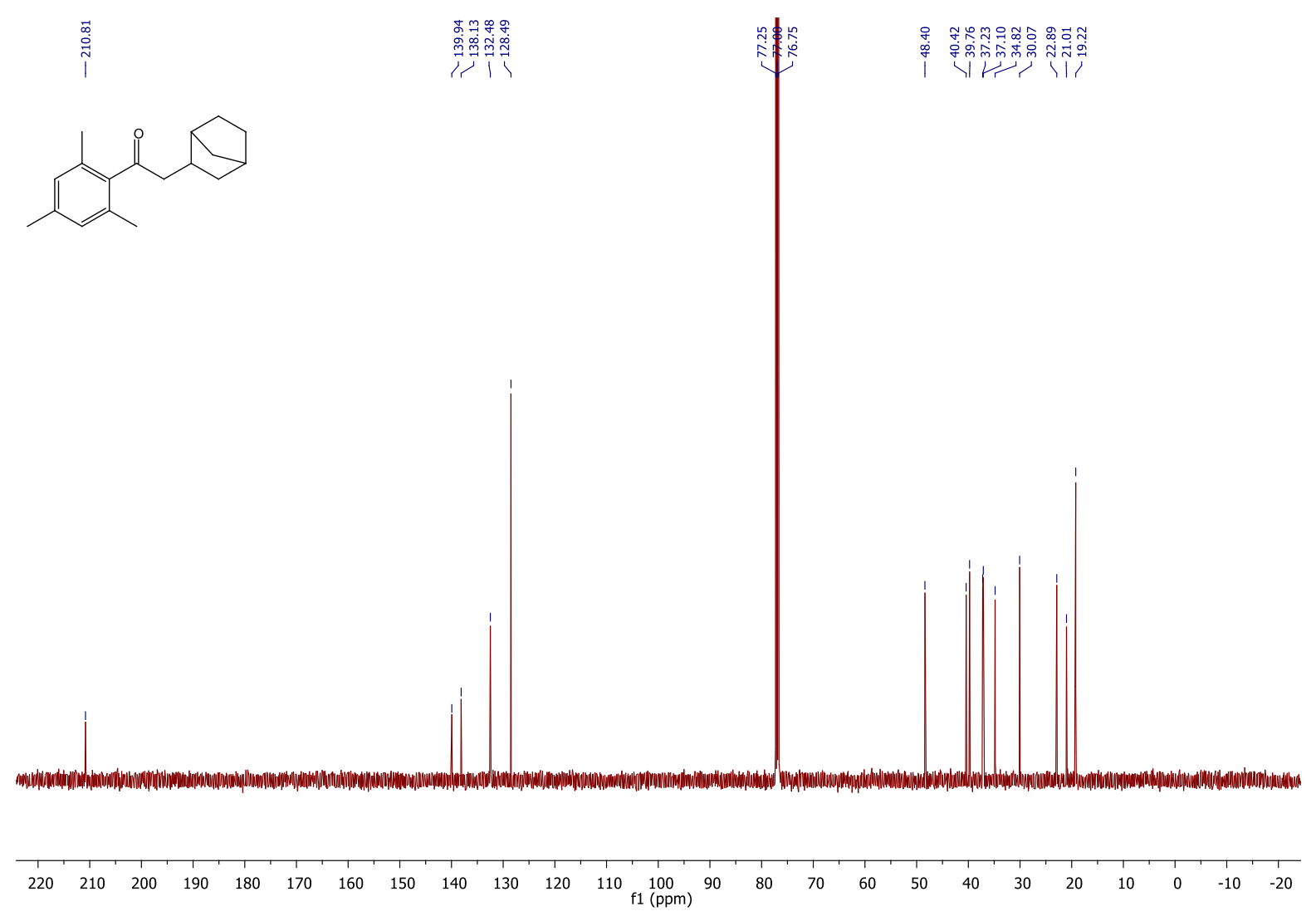

Figure S59: ${ }^{13} \mathrm{C}-\mathrm{NMR}(125 \mathrm{MHz})$ in $\mathrm{CDCl}_{3}$ of $1 \mathrm{z}$ 


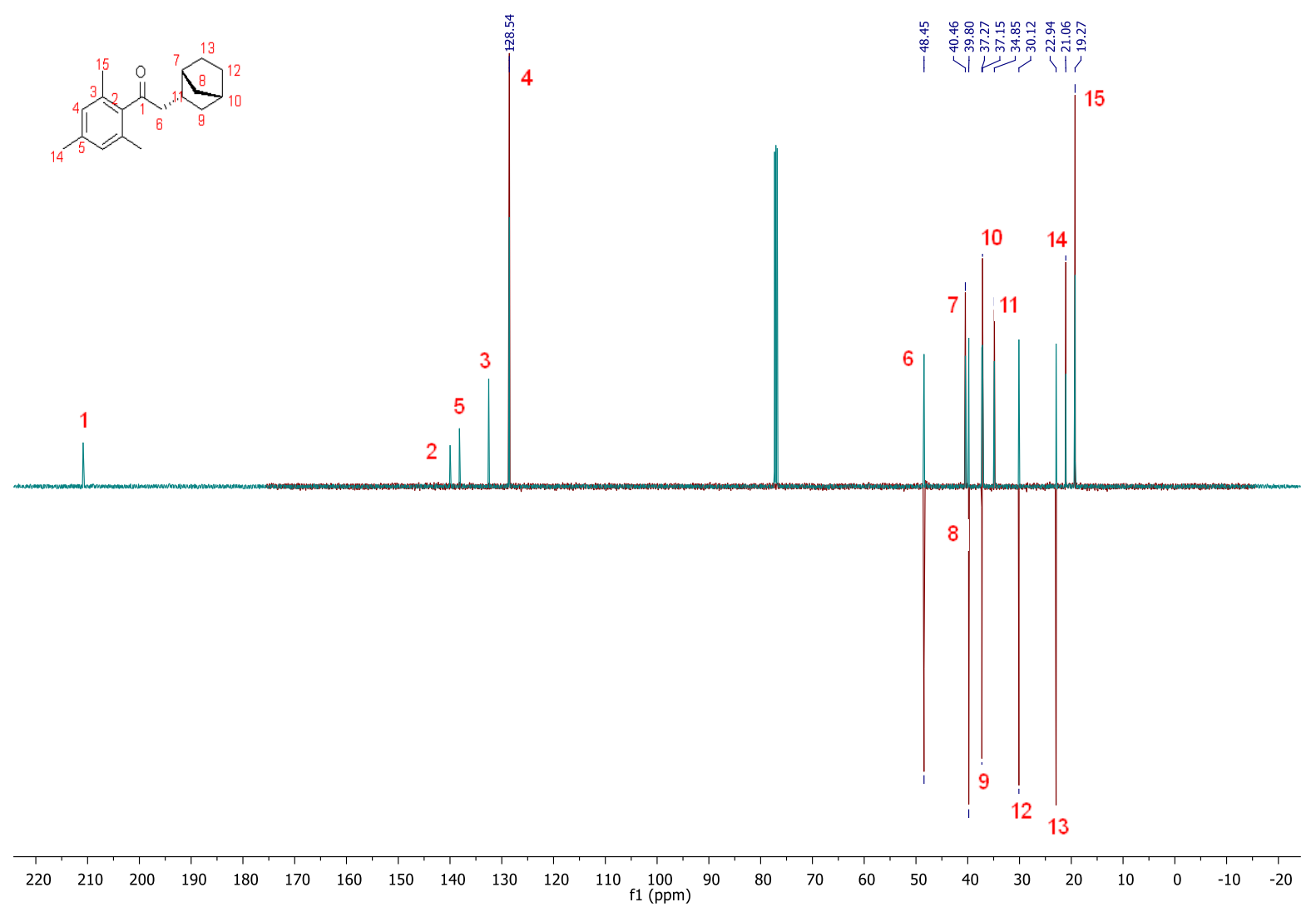

Figure S60: ${ }^{13} \mathrm{C}-\mathrm{NMR}(125 \mathrm{MHz})$ in $\mathrm{CDCl}_{3}+$ DEPT of $1 \mathrm{z}$

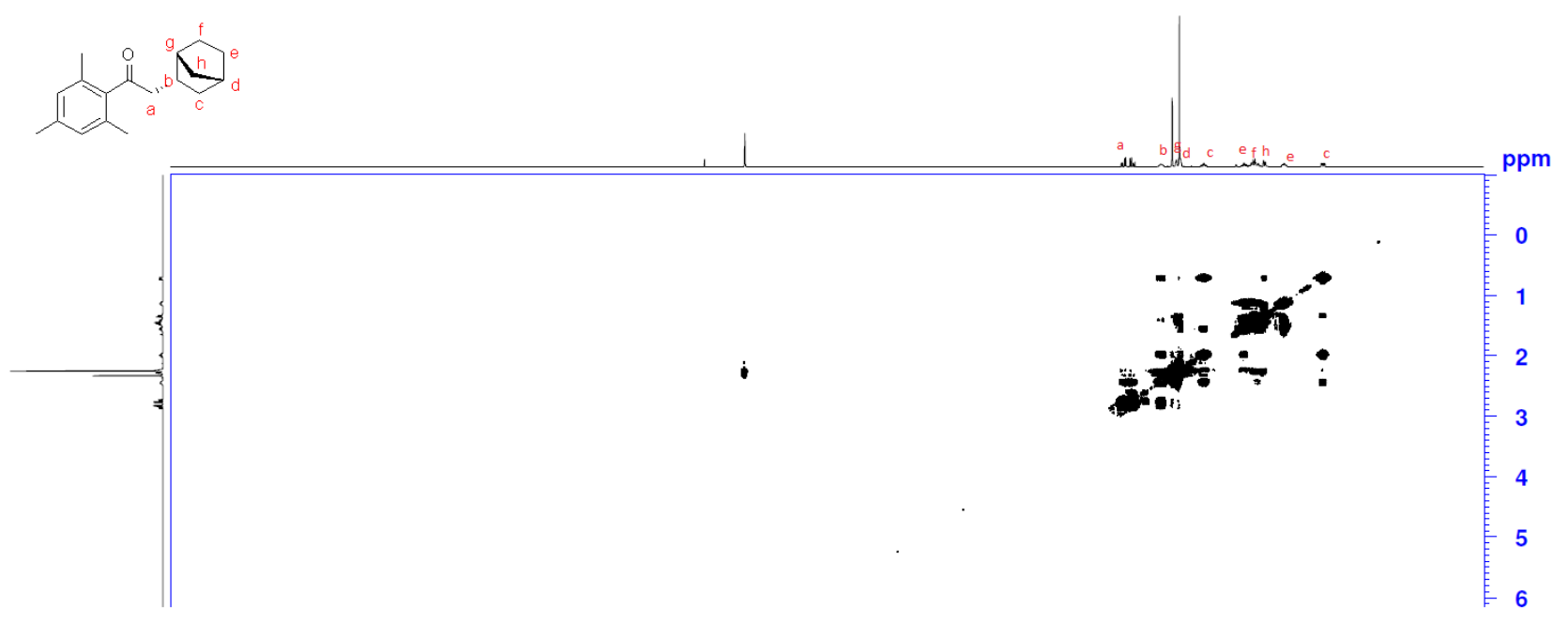

Figure S61: $\operatorname{COSY}(500 \mathrm{MHz})$ in $\mathrm{CDCl}_{3}$ of $1 z$ 


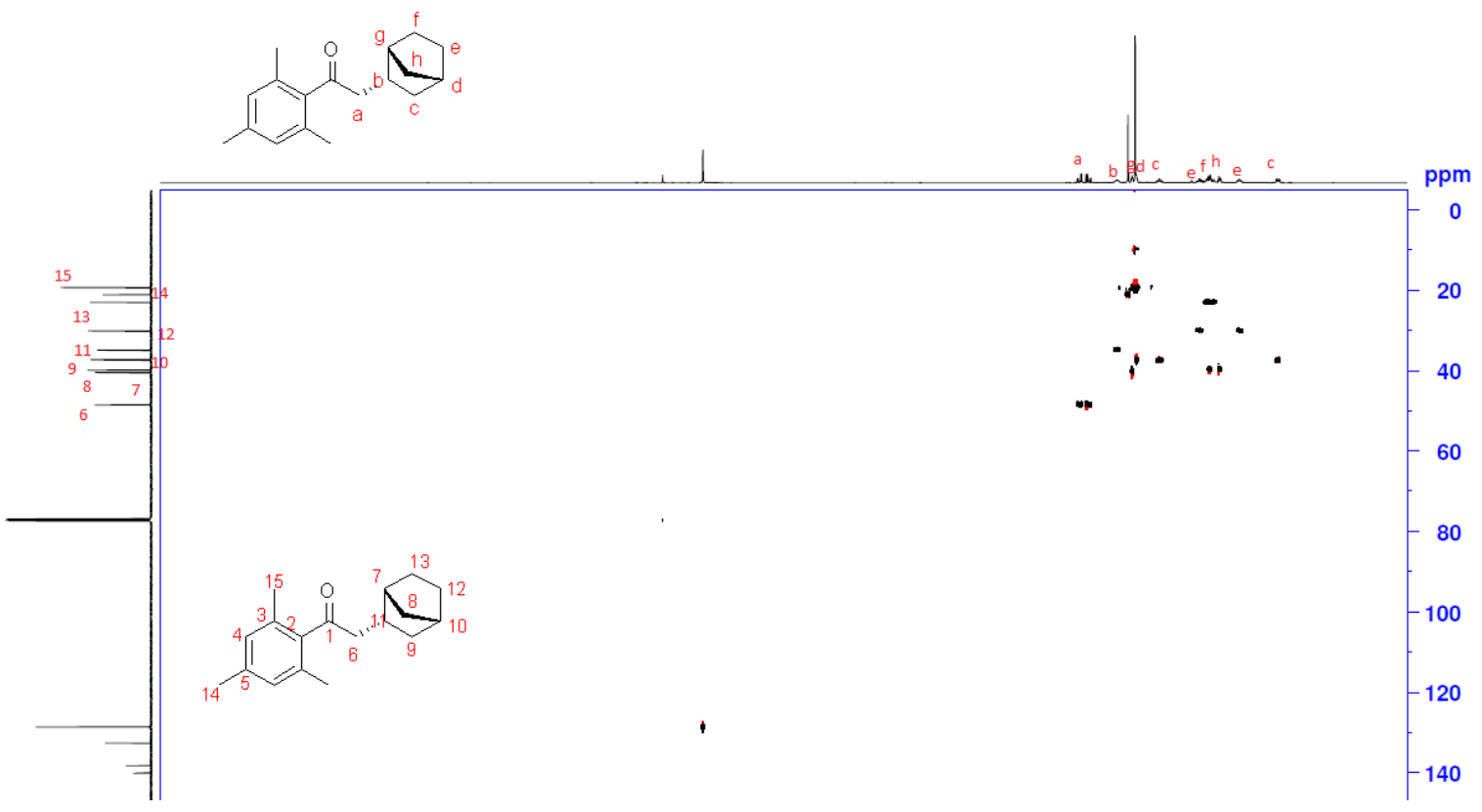

Figure S62: HSQC (500 MHz) in $\mathrm{CDCl}_{3}$ of $1 z$

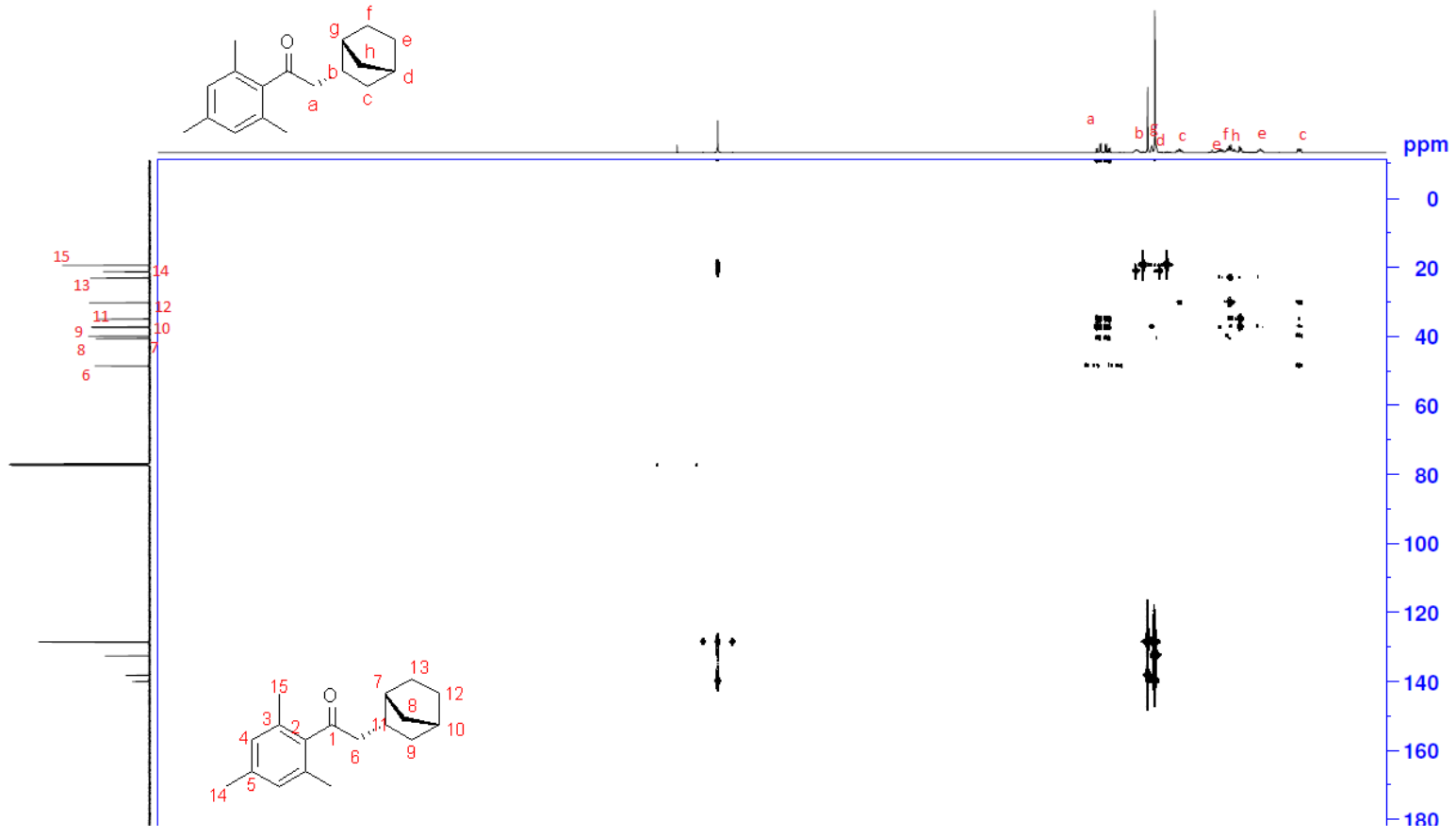

Figure S63: $\mathrm{HMBC}(500 \mathrm{MHz})$ in $\mathrm{CDCl}_{3}$ of $1 z$ 


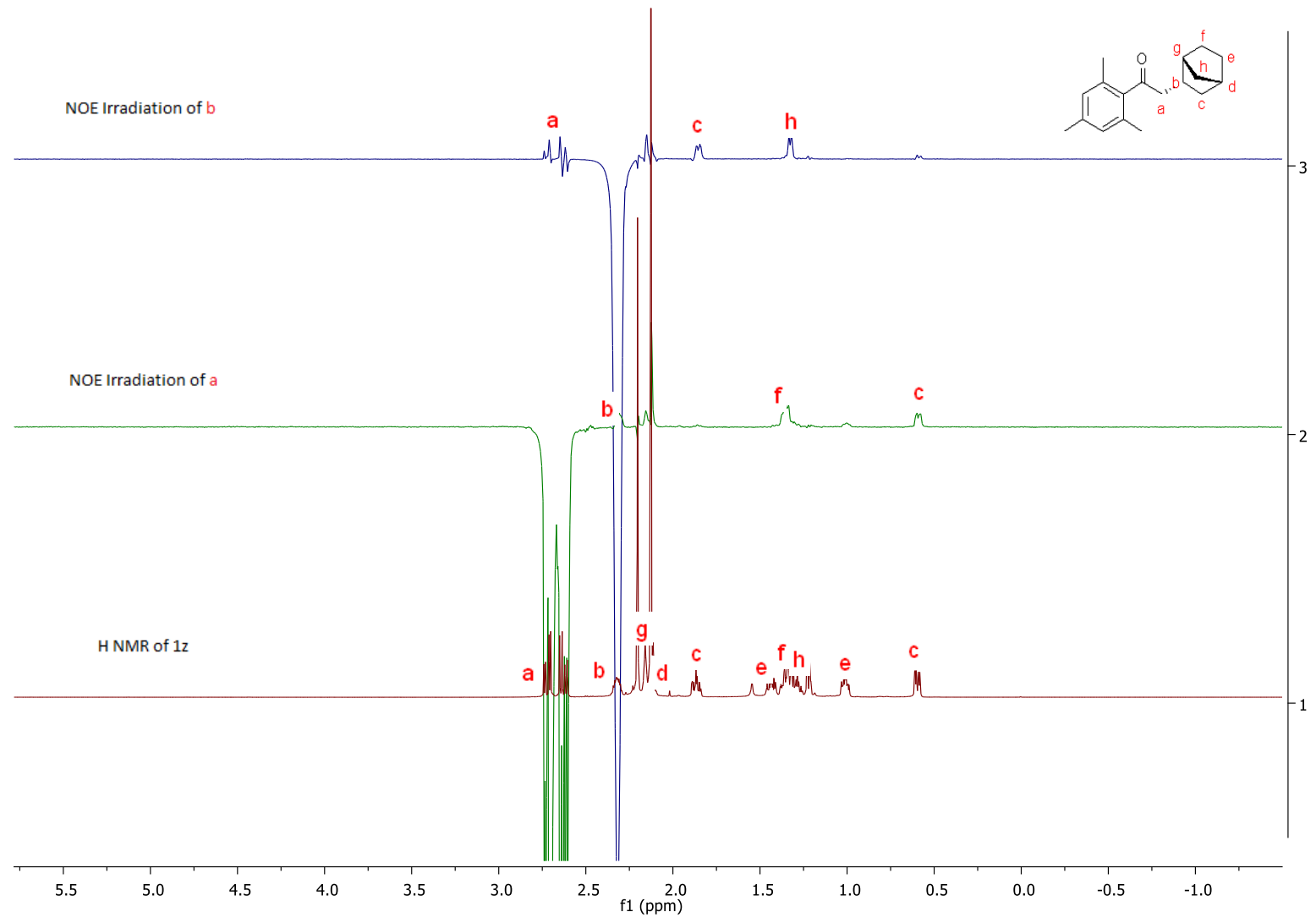

Figure S64: NOE Spectra $+{ }^{1} \mathrm{H}$ NMR $(500 \mathrm{MHz})$ in $\mathrm{CDCl}_{3}$ of $1 \mathrm{z}$ 


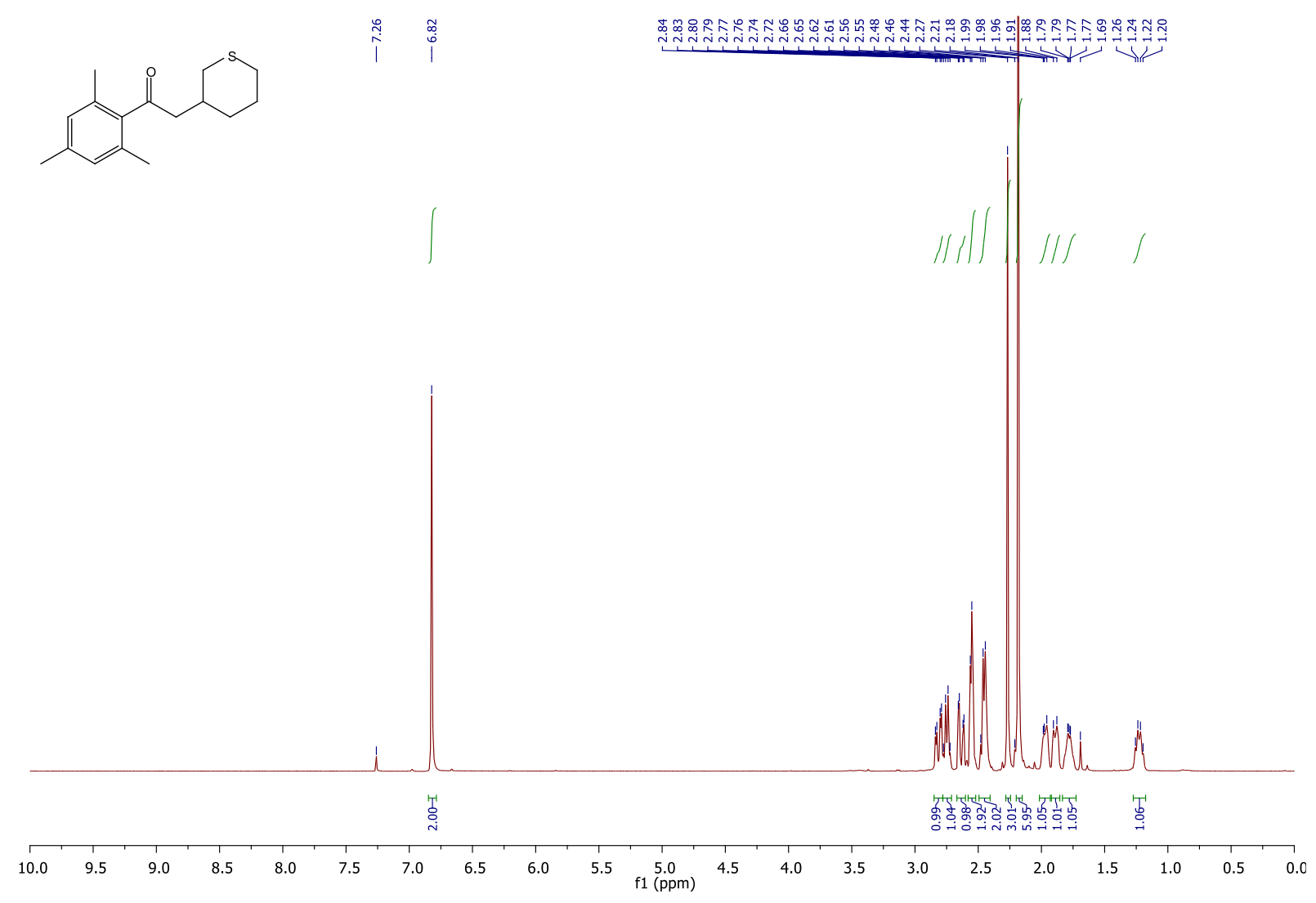

Figure S65: ${ }^{1} \mathrm{H}-\mathrm{NMR}(500 \mathrm{MHz})$ in $\mathrm{CDCl}_{3}$ of $1 \mathrm{aa}$

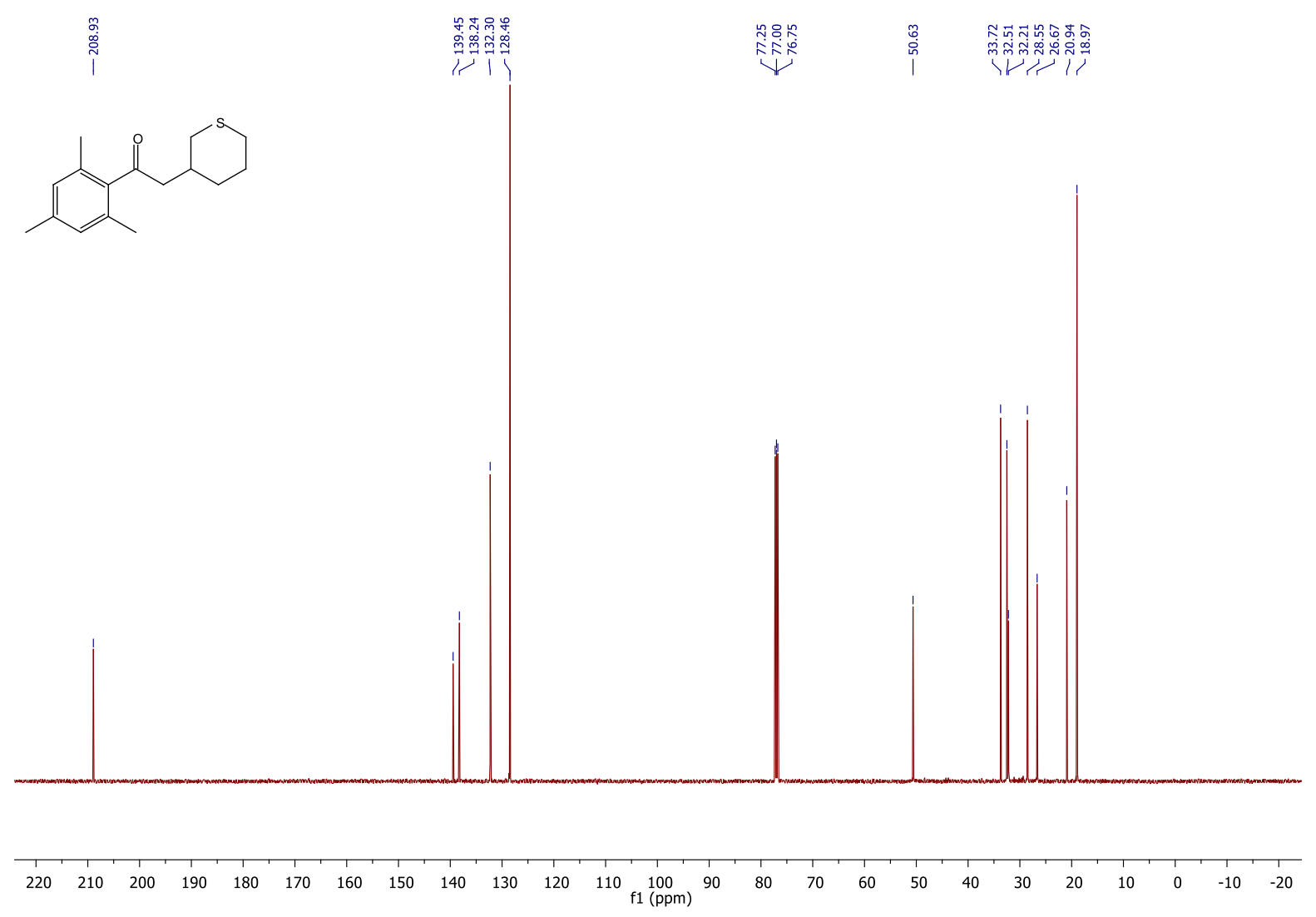

Figure S66: ${ }^{13} \mathrm{C}-\mathrm{NMR}$ (125 MHz) in $\mathrm{CDCl}_{3}$ of 1 aa 


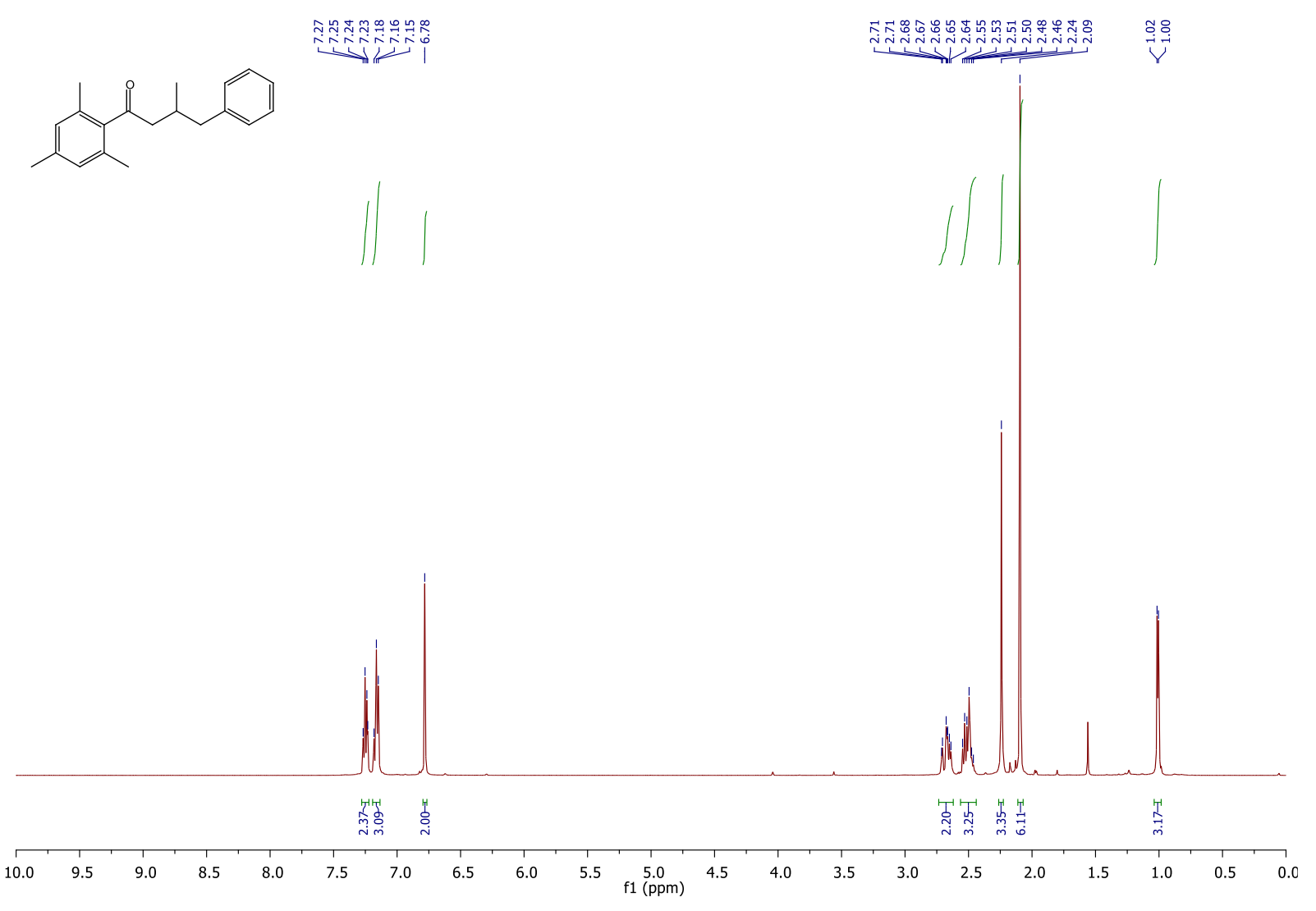

Figure S67: ${ }^{1} \mathrm{H}-\mathrm{NMR}(500 \mathrm{MHz})$ in $\mathrm{CDCl}_{3}$ of $1 \mathrm{ab}$
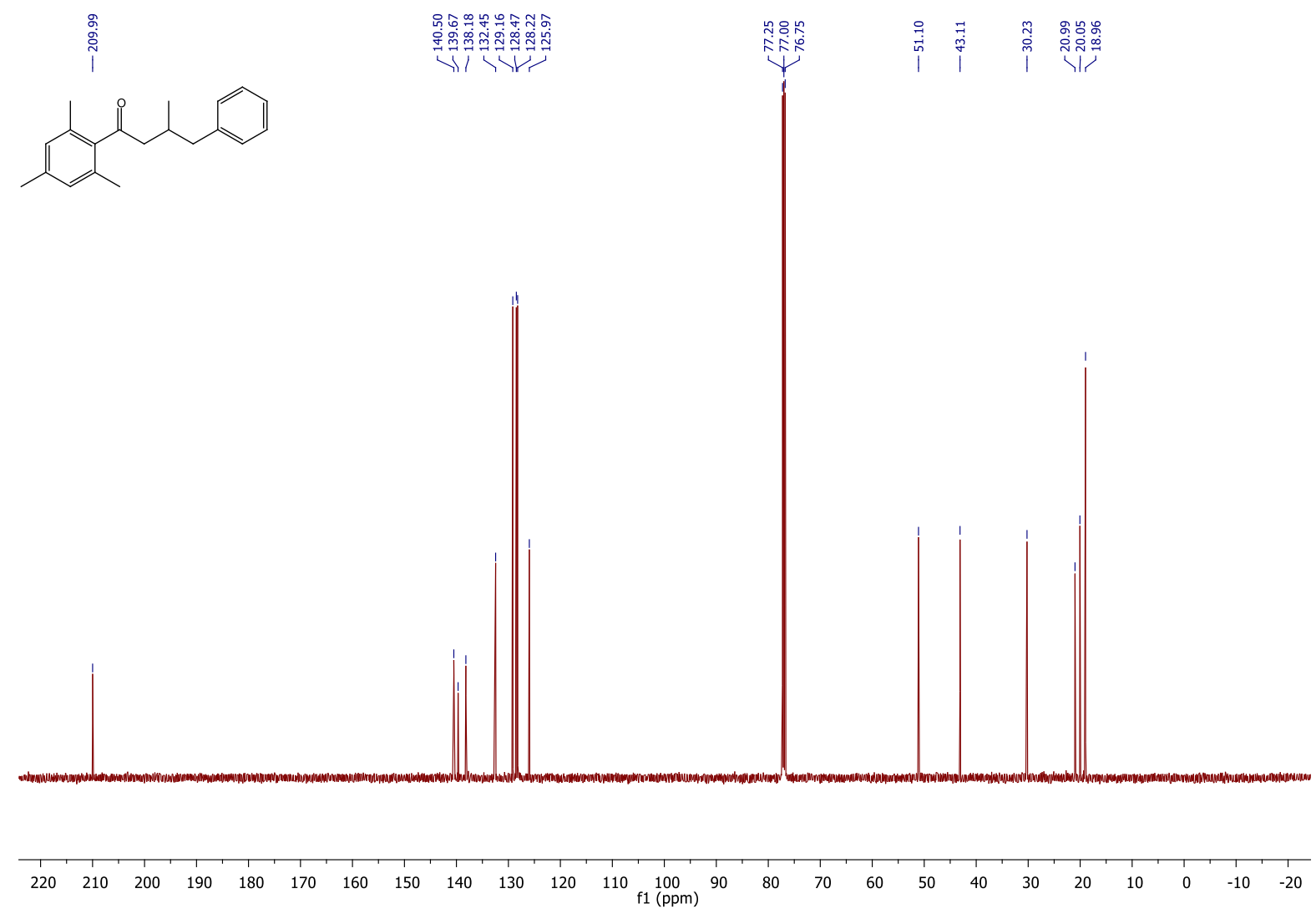

Figure S68: ${ }^{13} \mathrm{C}-\mathrm{NMR}(125 \mathrm{MHz})$ in $\mathrm{CDCl}_{3}$ of $1 \mathrm{ab}$

S-57 

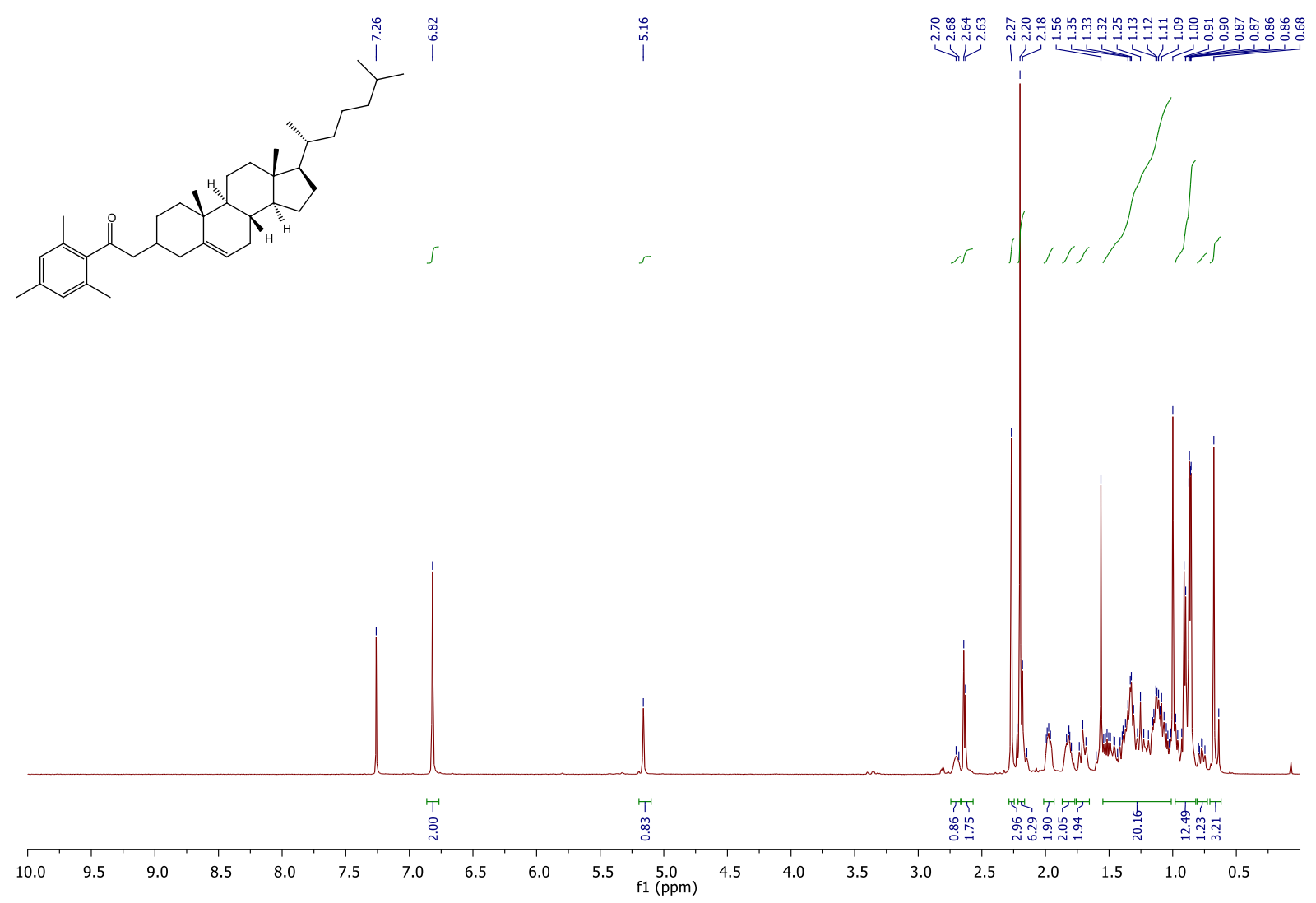

Figure S69: ${ }^{1} \mathrm{H}-\mathrm{NMR}(500 \mathrm{MHz})$ in $\mathrm{CDCl}_{3}$ of $1 \mathrm{ac}$
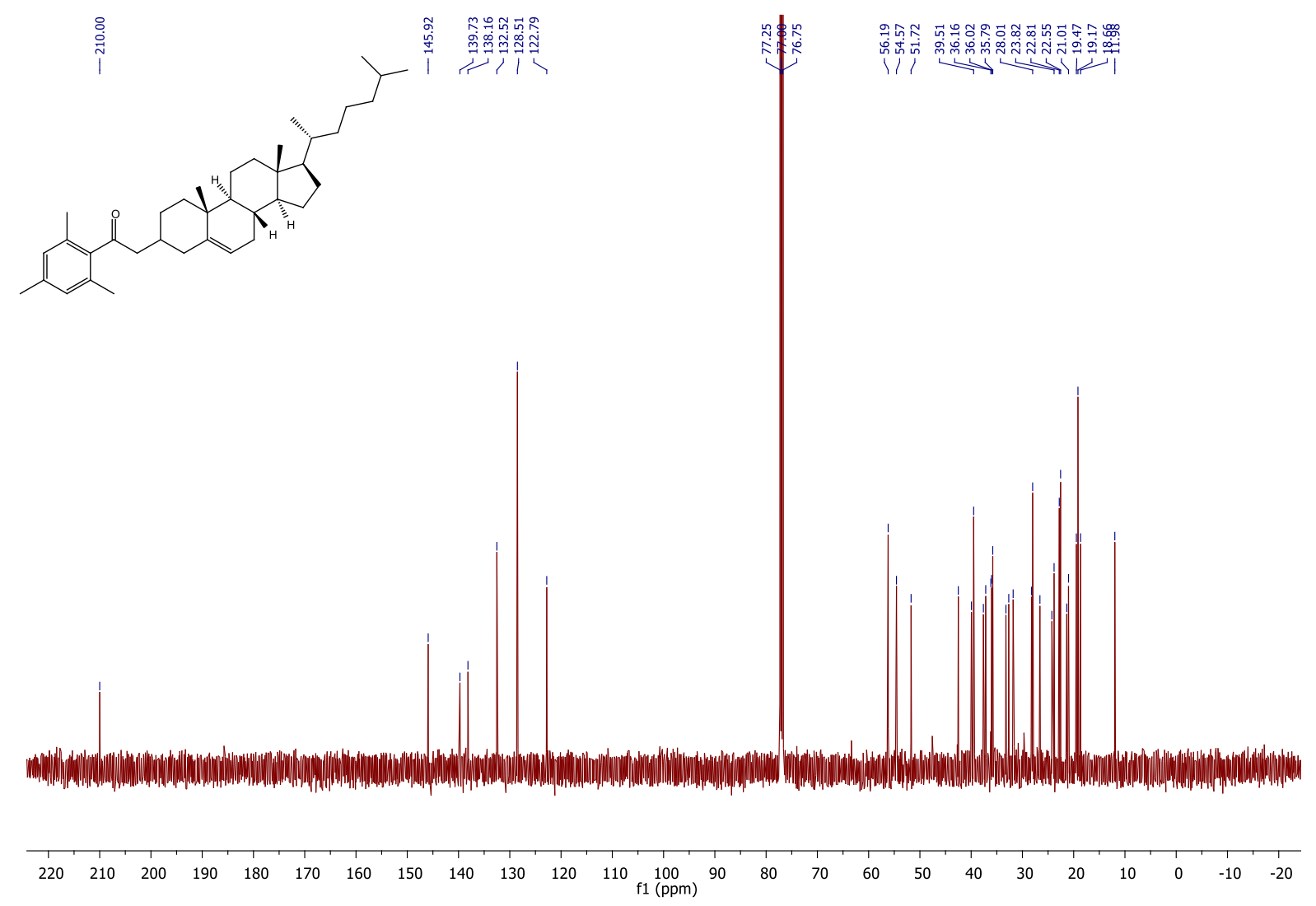

Figure S70: ${ }^{13} \mathrm{C}-\mathrm{NMR}(125 \mathrm{MHz})$ in $\mathrm{CDCl}_{3}$ of $1 \mathrm{ac}$ 


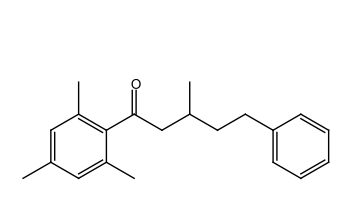

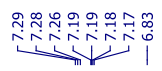

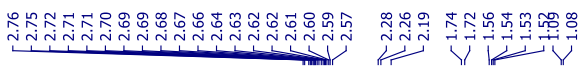
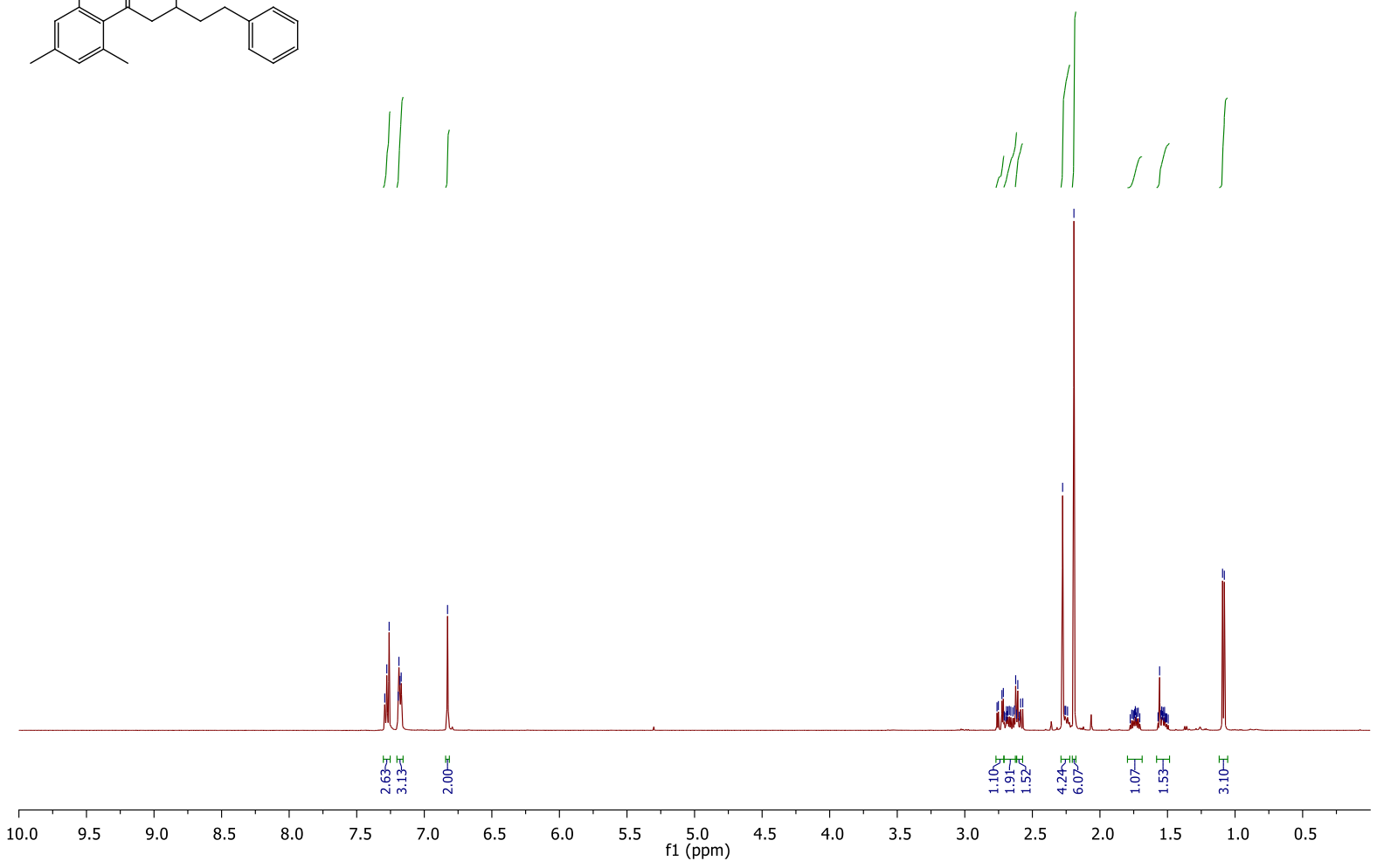

Figure S71: ${ }^{1} \mathrm{H}-\mathrm{NMR}(500 \mathrm{MHz})$ in $\mathrm{CDCl}_{3}$ of $1 \mathrm{ad}$
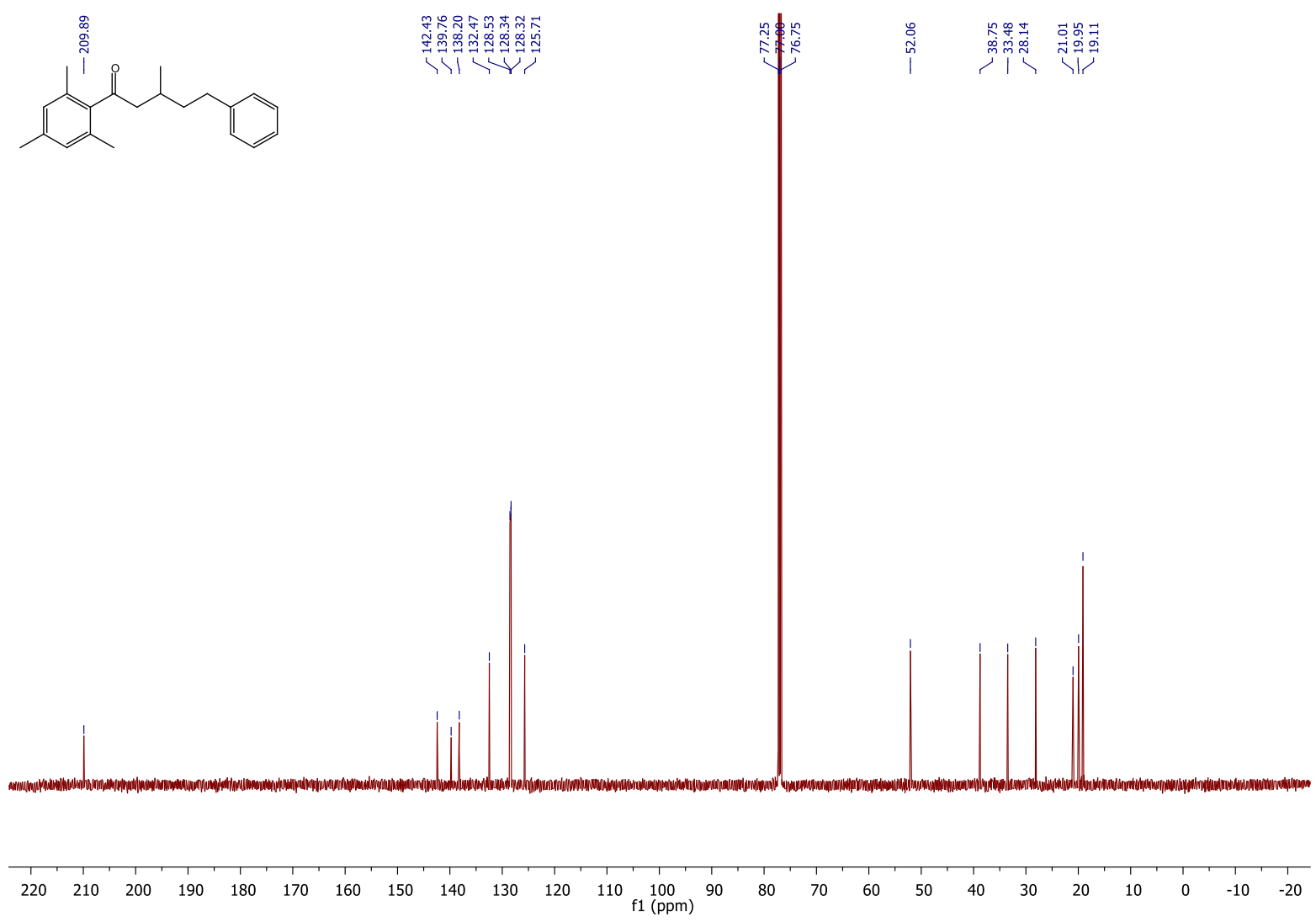

Figure S72: ${ }^{13} \mathrm{C}-\mathrm{NMR}(125 \mathrm{MHz})$ in $\mathrm{CDCl}_{3}$ of $1 \mathrm{ad}$

S-59 


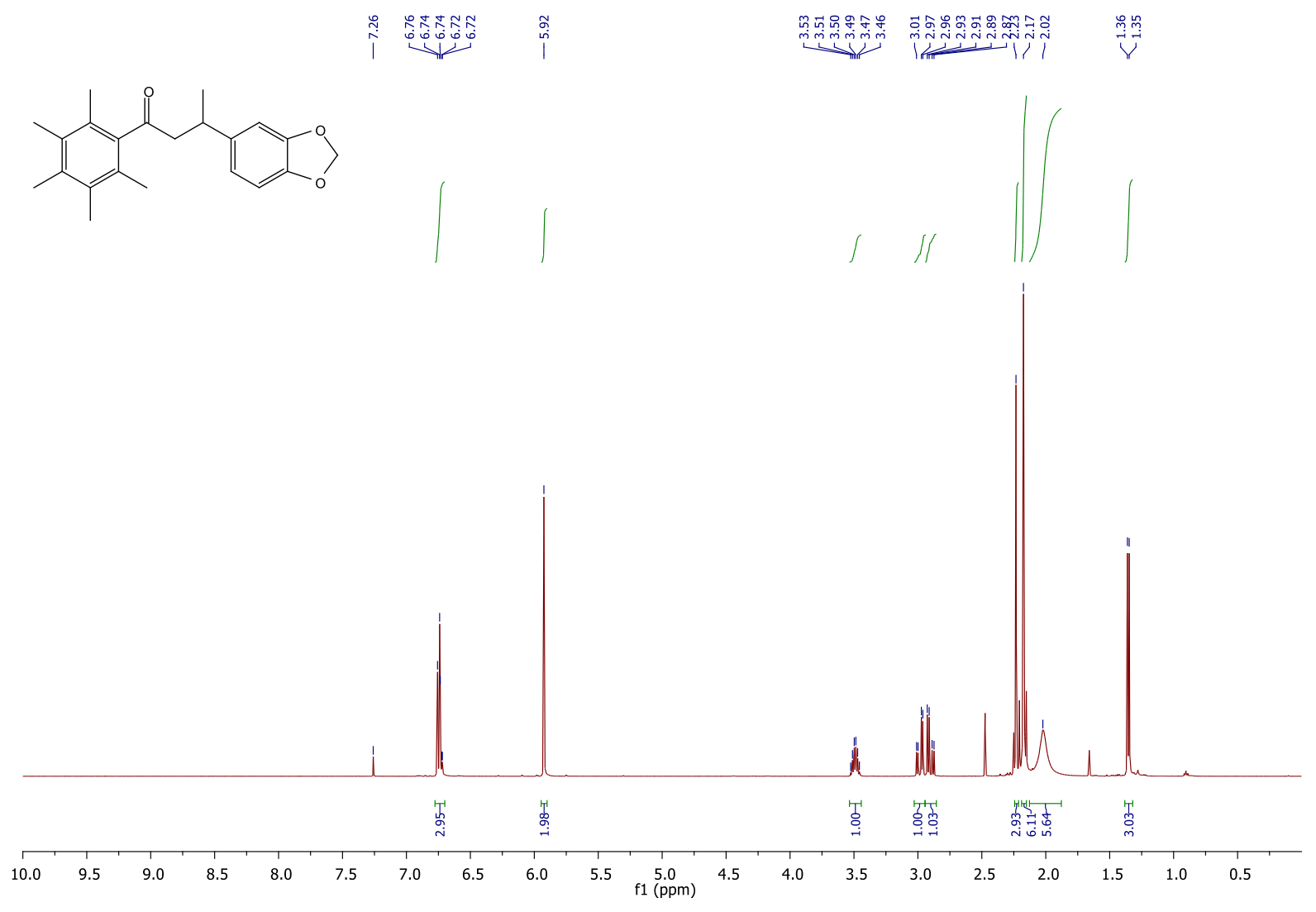

Figure S73: ${ }^{1} \mathrm{H}-\mathrm{NMR}(500 \mathrm{MHz})$ in $\mathrm{CDCl}_{3}$ of $2 \mathrm{~b}$
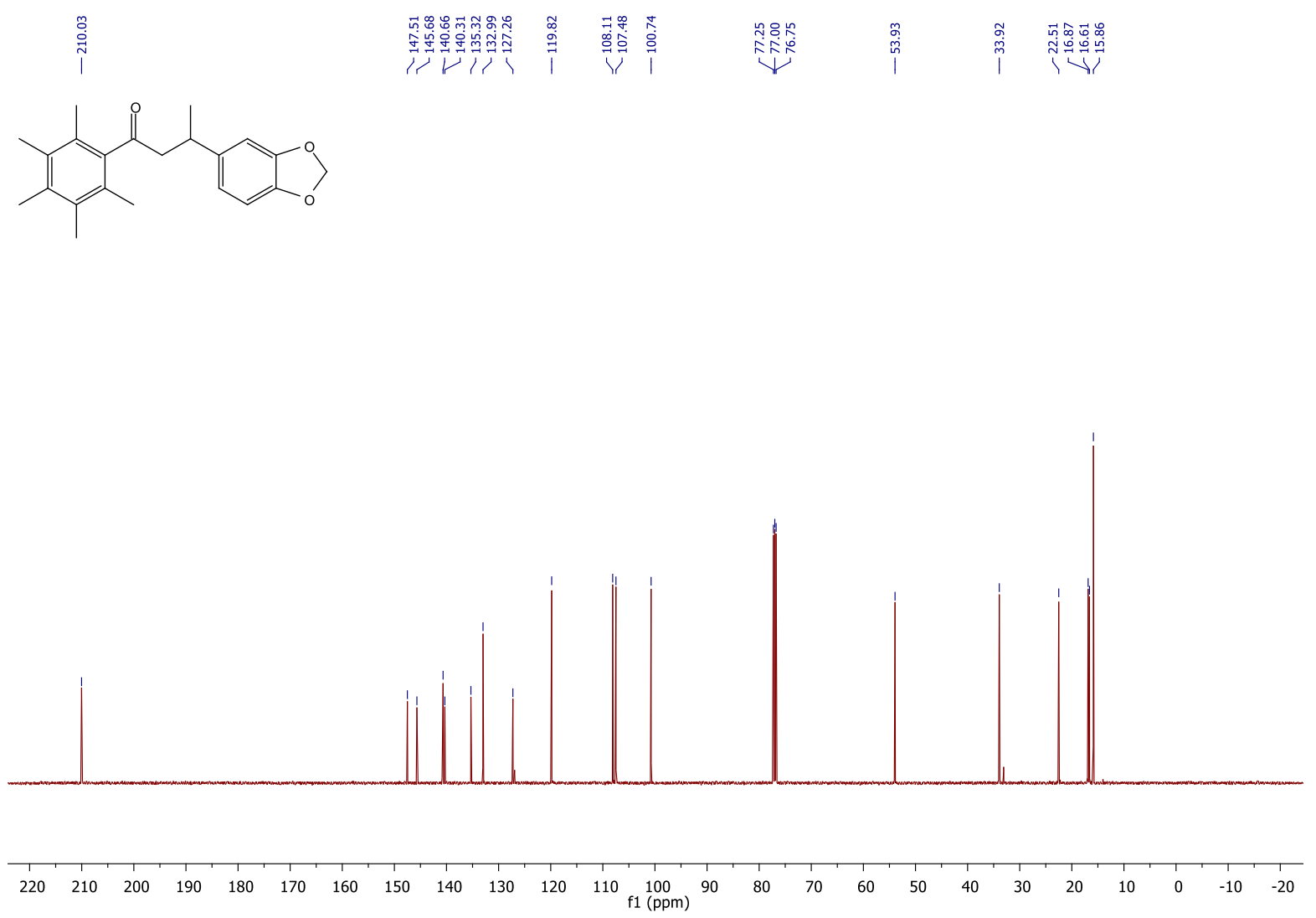

Figure S74: ${ }^{13} \mathrm{C}$-NMR (125 MHz) in $\mathrm{CDCl}_{3}$ of $2 \mathrm{~b}$

S-60 


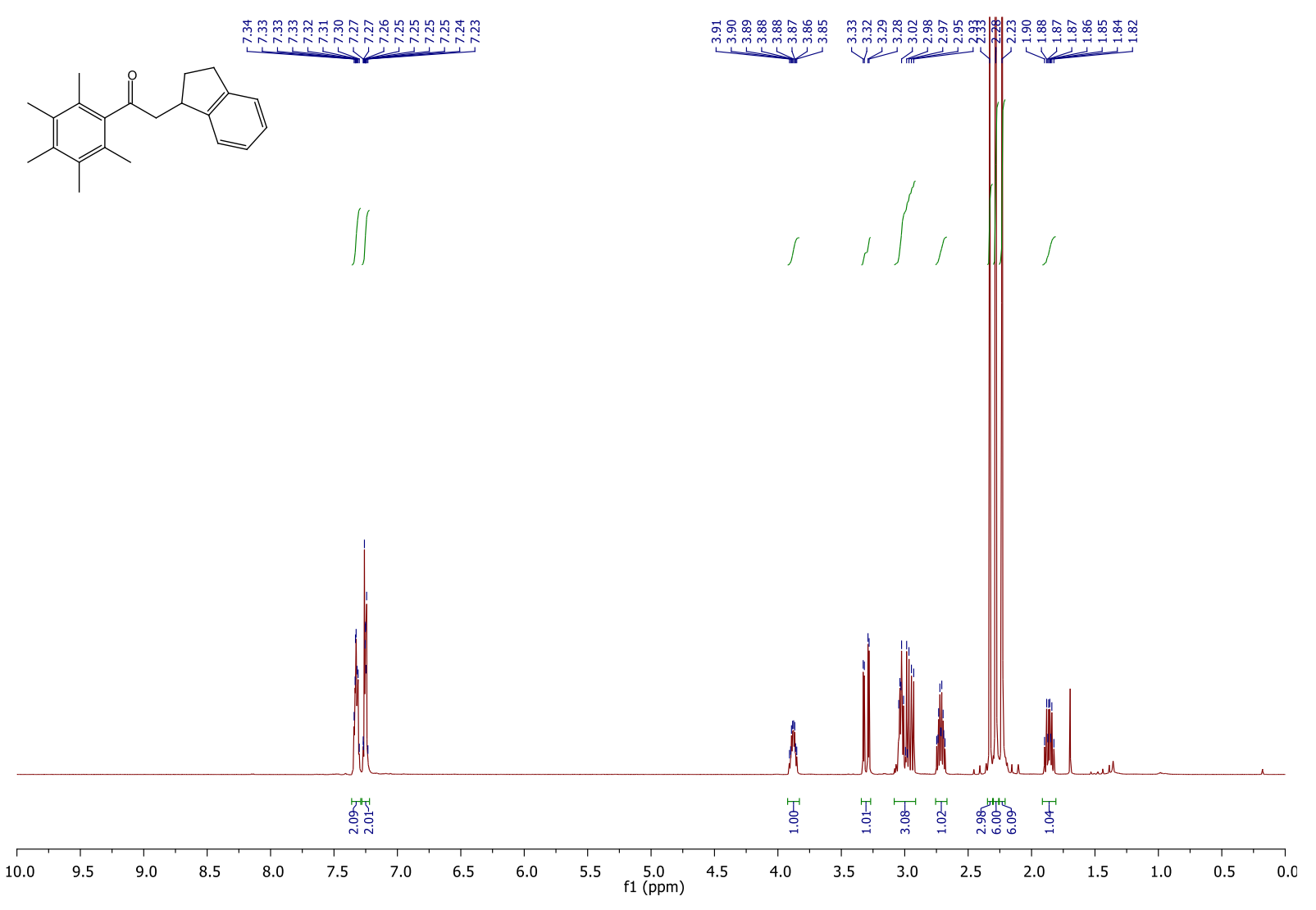

Figure S75: ${ }^{1} \mathrm{H}-\mathrm{NMR}(500 \mathrm{MHz})$ in $\mathrm{CDCl}_{3}$ of $2 \mathrm{C}$
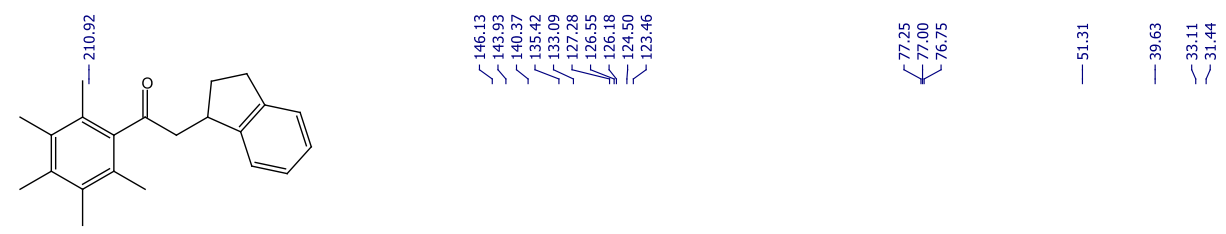

|ำ
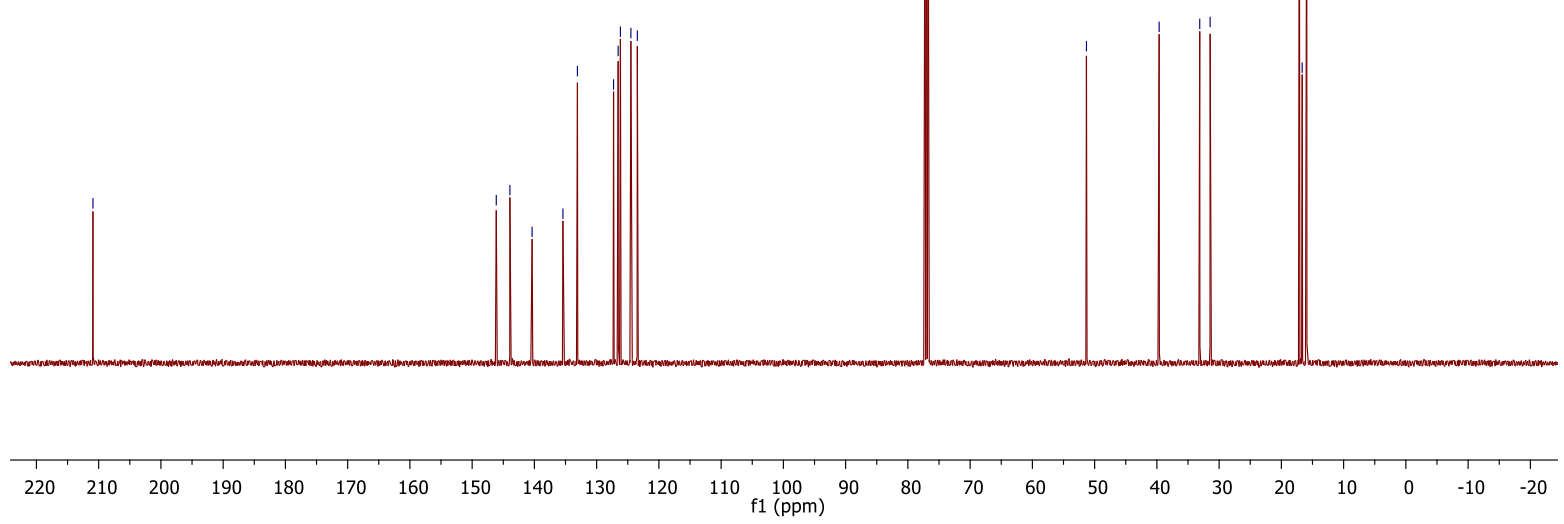

Figure S76: ${ }^{13} \mathrm{C}-\mathrm{NMR}(125 \mathrm{MHz})$ in $\mathrm{CDCl}_{3}$ of $2 \mathrm{C}$

S-61 


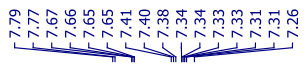

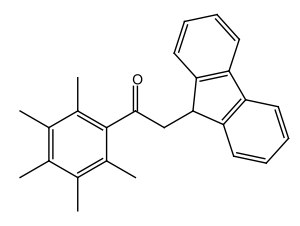

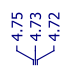

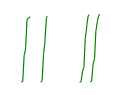

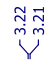

ปู่

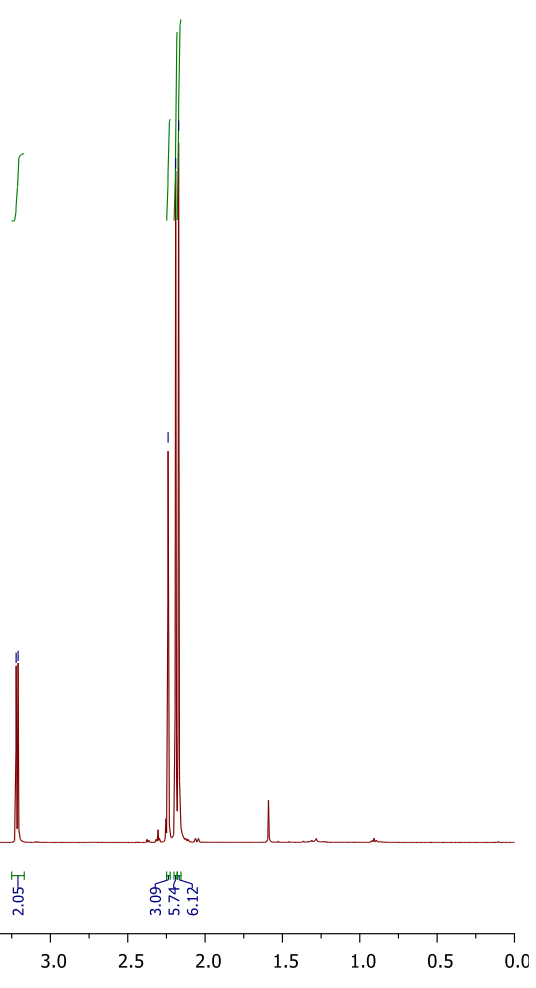

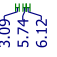

崇

Figure S77: ${ }^{1} \mathrm{H}-\mathrm{NMR}(500 \mathrm{MHz})$ in $\mathrm{CDCl}_{3}$ of $2 \mathrm{~d}$
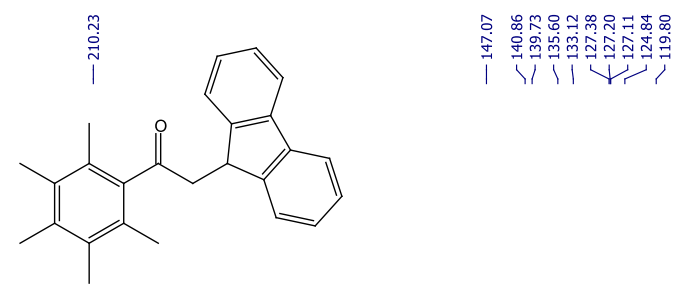

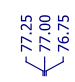

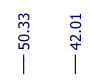

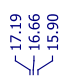
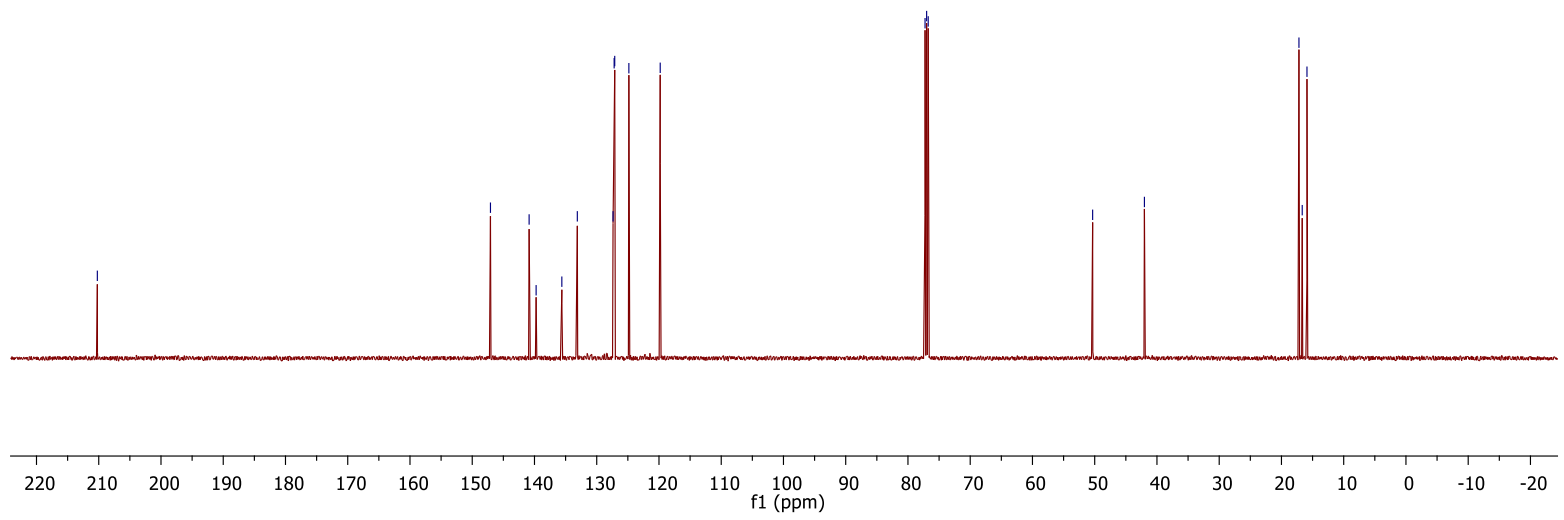

Figure S78: ${ }^{13} \mathrm{C}-\mathrm{NMR}(125 \mathrm{MHz})$ in $\mathrm{CDCl}_{3}$ of $2 \mathrm{~d}$

S-62 


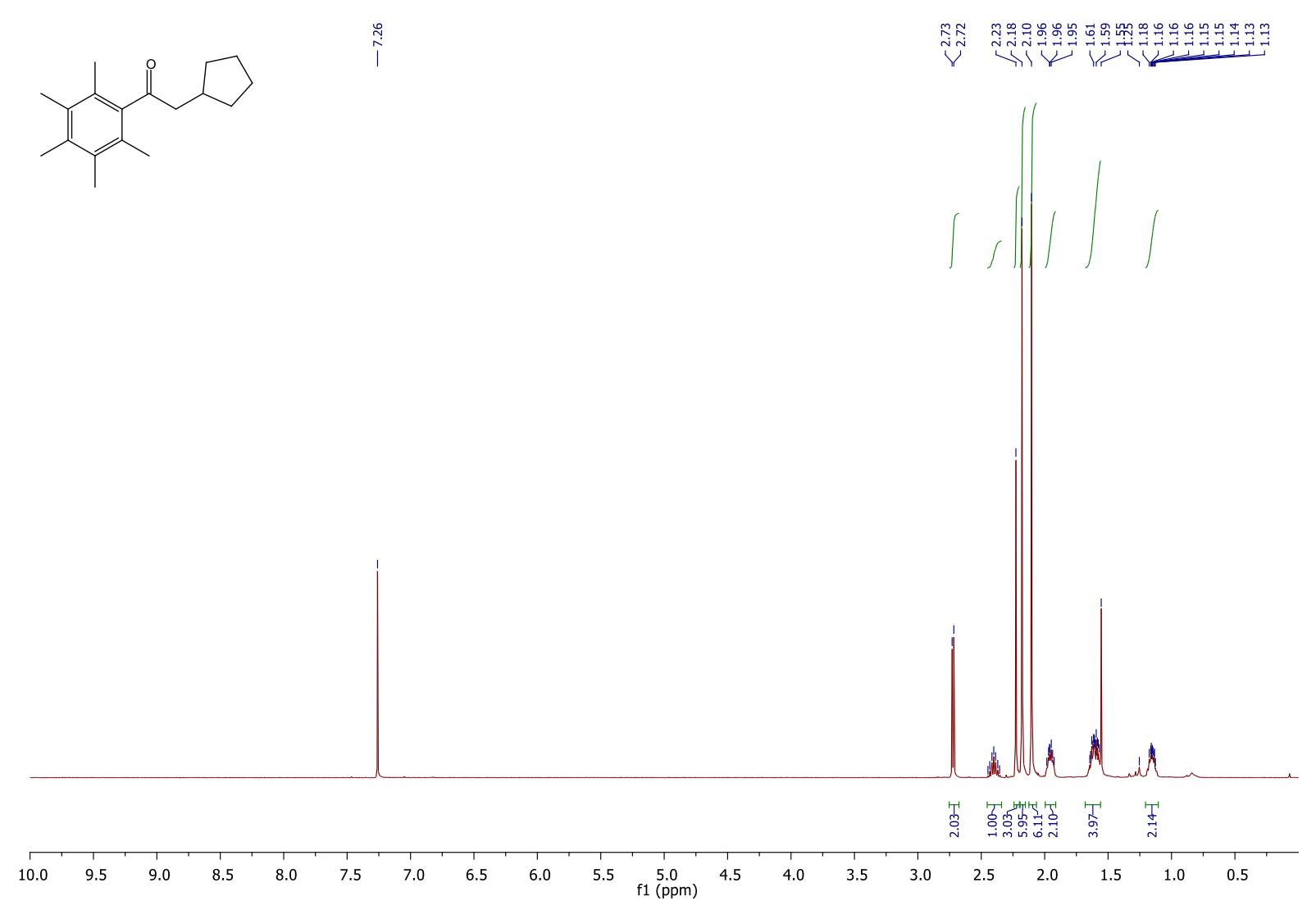

Figure S79: ${ }^{1} \mathrm{H}-\mathrm{NMR}(500 \mathrm{MHz})$ in $\mathrm{CDCl}_{3}$ of $2 \mathrm{e}$

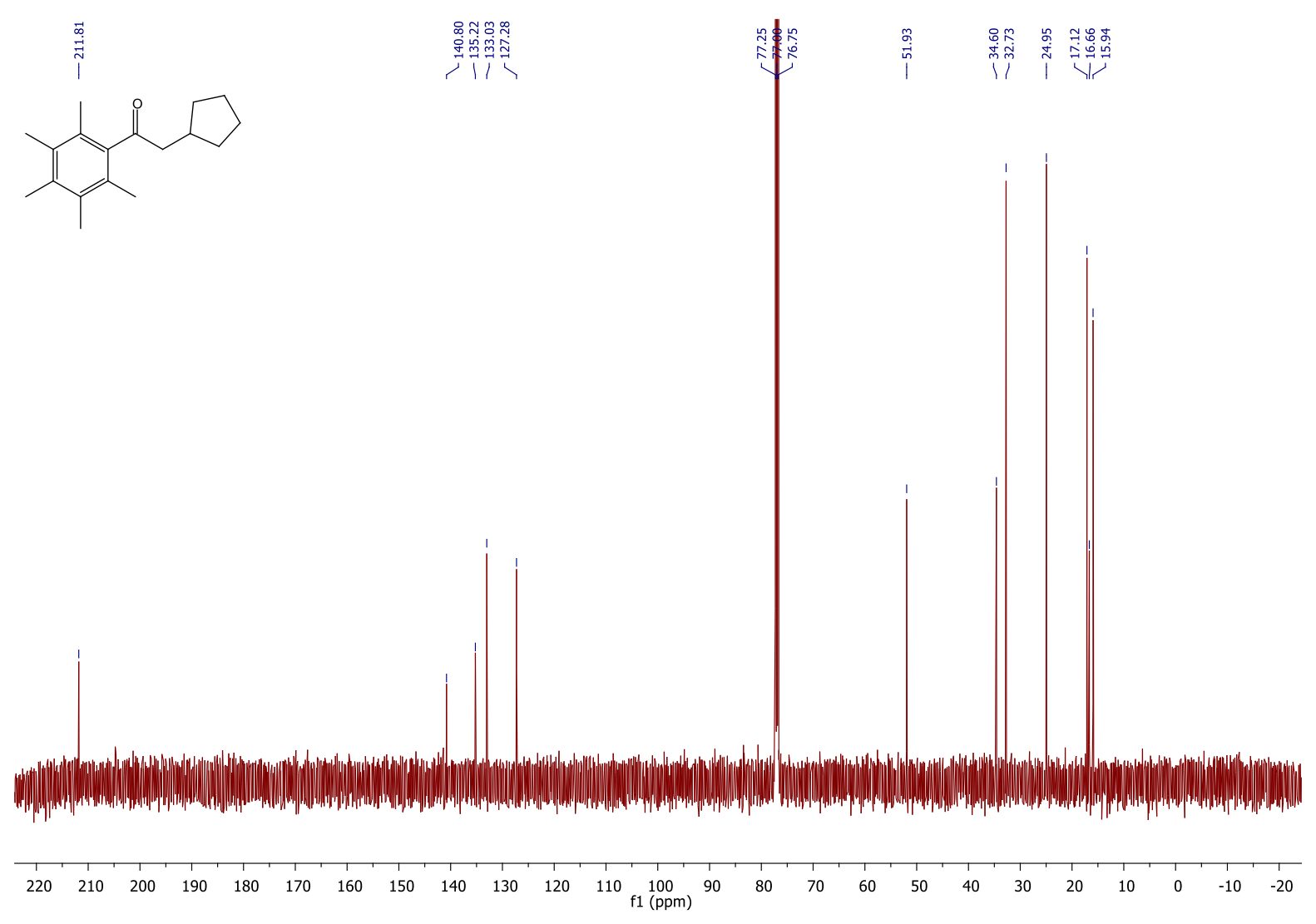

Figure S80: ${ }^{13} \mathrm{C}$-NMR (125 MHz) in $\mathrm{CDCl}_{3}$ of $2 \mathrm{e}$ 


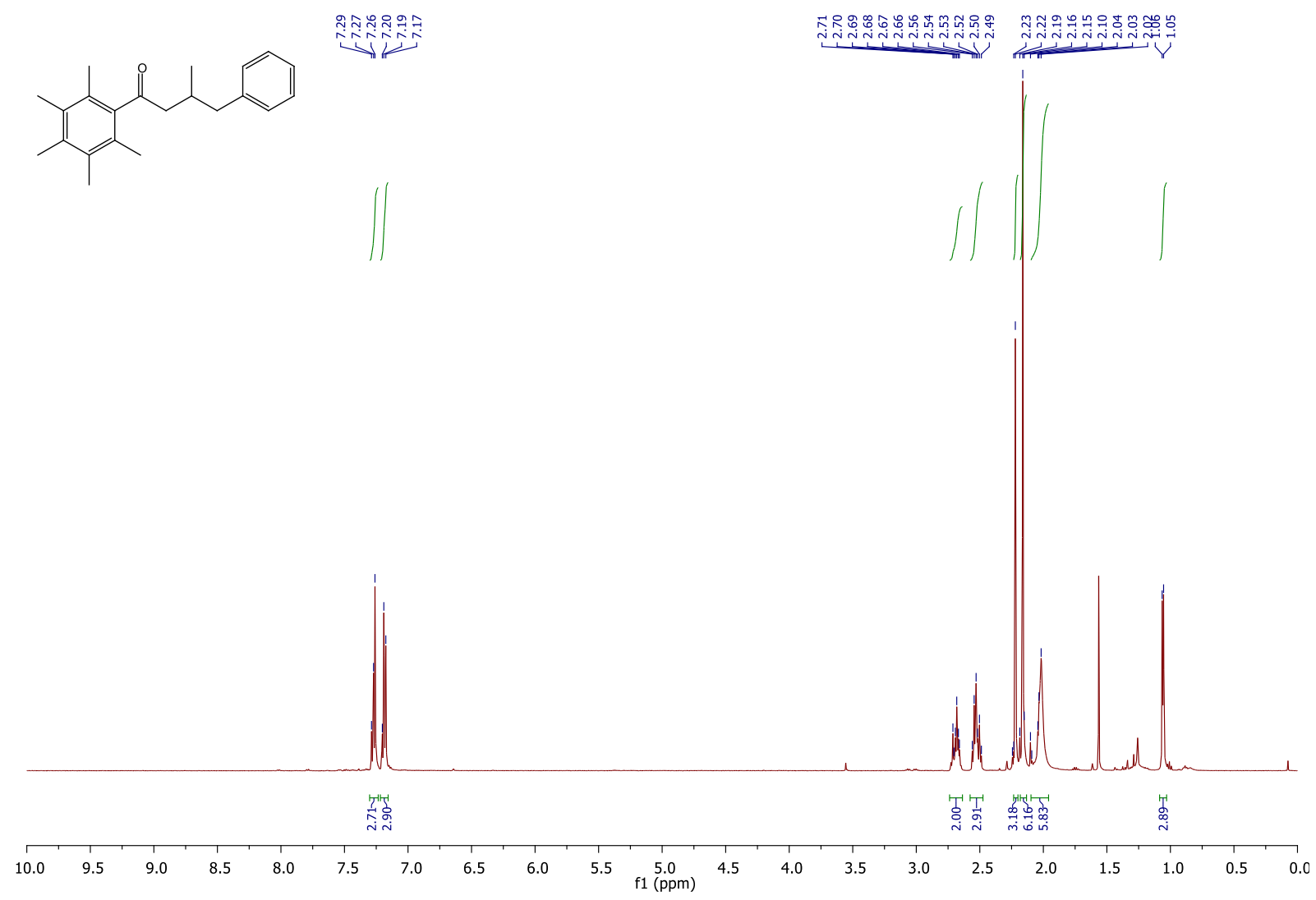

Figure S81: ${ }^{1} \mathrm{H}-\mathrm{NMR}(500 \mathrm{MHz})$ in $\mathrm{CDCl}_{3}$ of $2 \mathrm{f}$

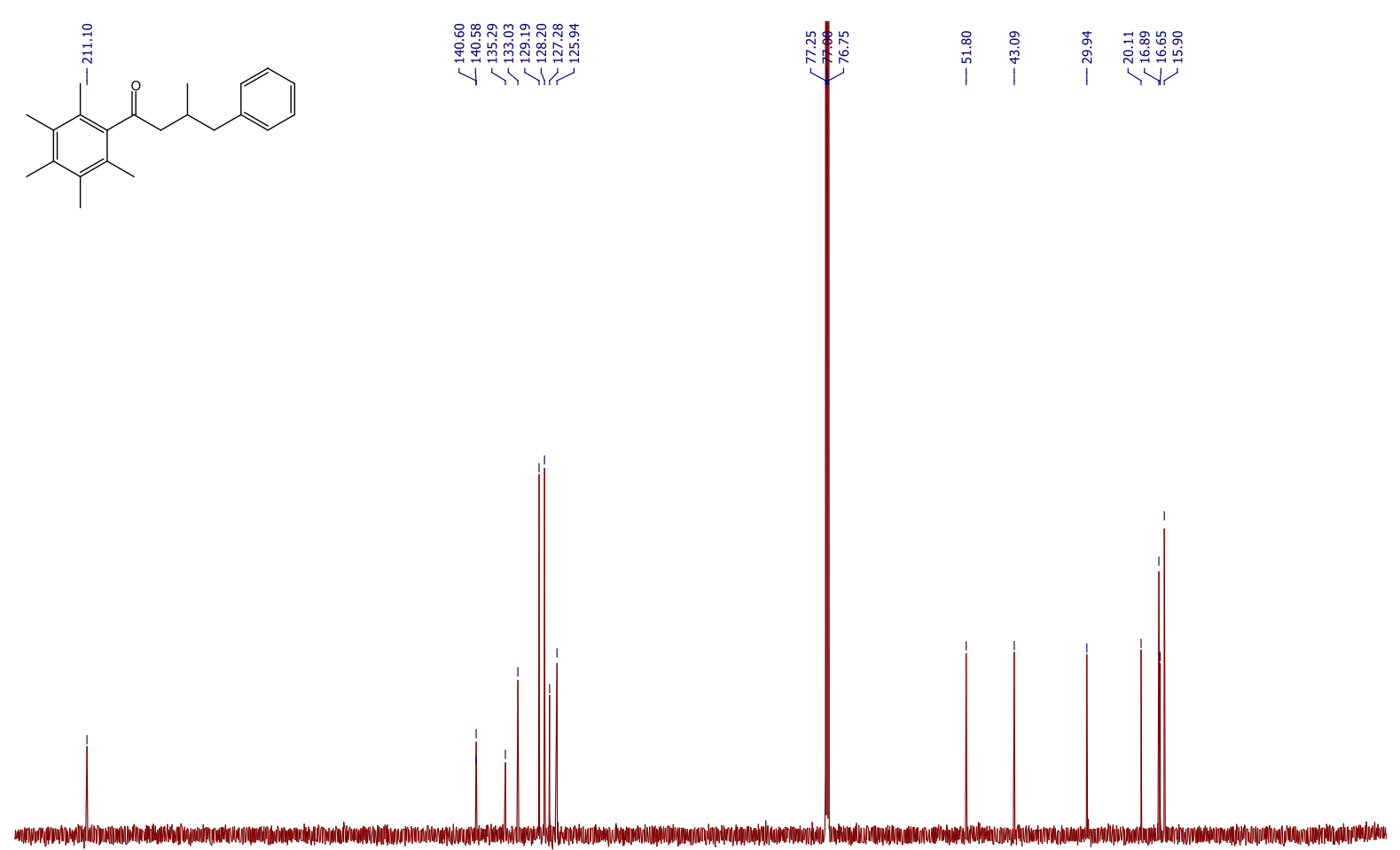

$\begin{array}{llllllllllllllllllllllllllllll}220 & 210 & 200 & 190 & 180 & 170 & 160 & 150 & 140 & 130 & 120 & 110 & 100 & 90 & 80 & 70 & 60 & 50 & 40 & 30 & 20 & 10 & 0 & -10 & -20\end{array}$

Figure S82: ${ }^{13} \mathrm{C}$-NMR (125 MHz) in $\mathrm{CDCl}_{3}$ of $2 \mathrm{f}$ 


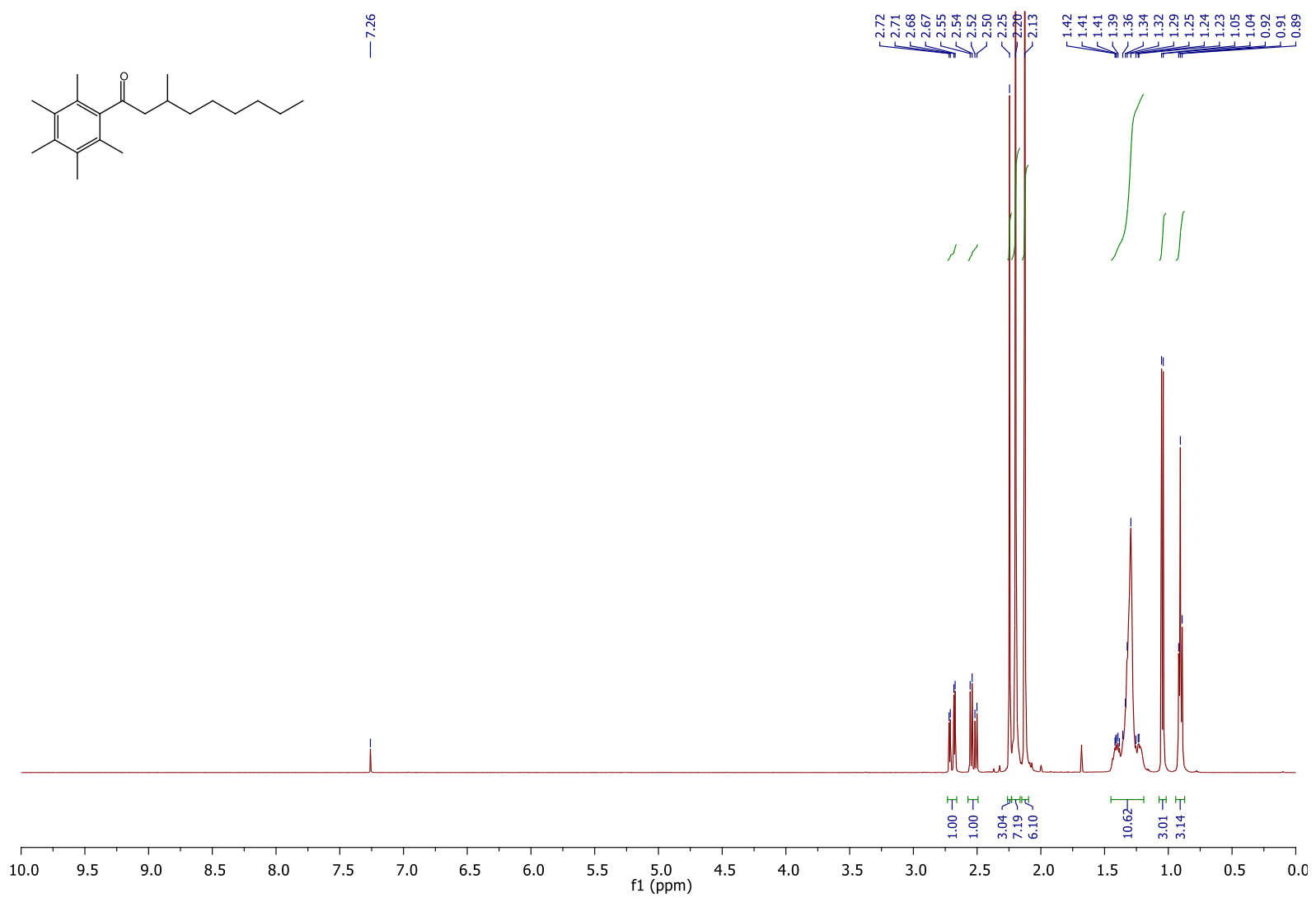

Figure S83: ${ }^{1} \mathrm{H}-\mathrm{NMR}(500 \mathrm{MHz})$ in $\mathrm{CDCl}_{3}$ of $2 \mathrm{~g}$
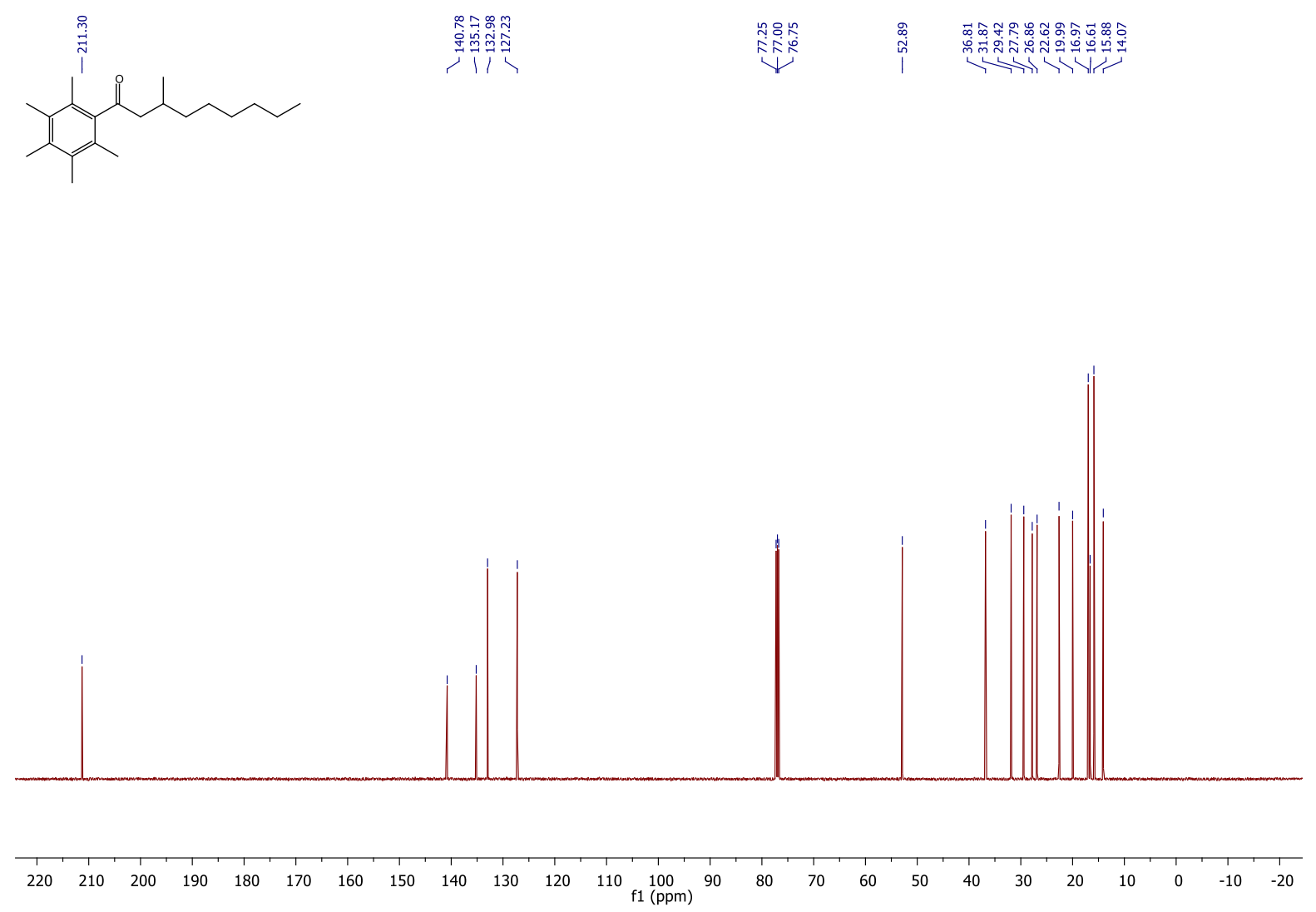

Figure S84: ${ }^{13} \mathrm{C}-\mathrm{NMR}(125 \mathrm{MHz})$ in $\mathrm{CDCl}_{3}$ of $\mathbf{2 g}$

S-65 


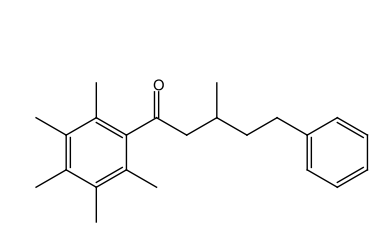

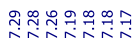

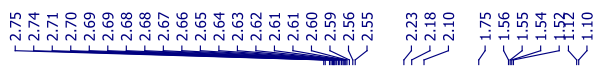
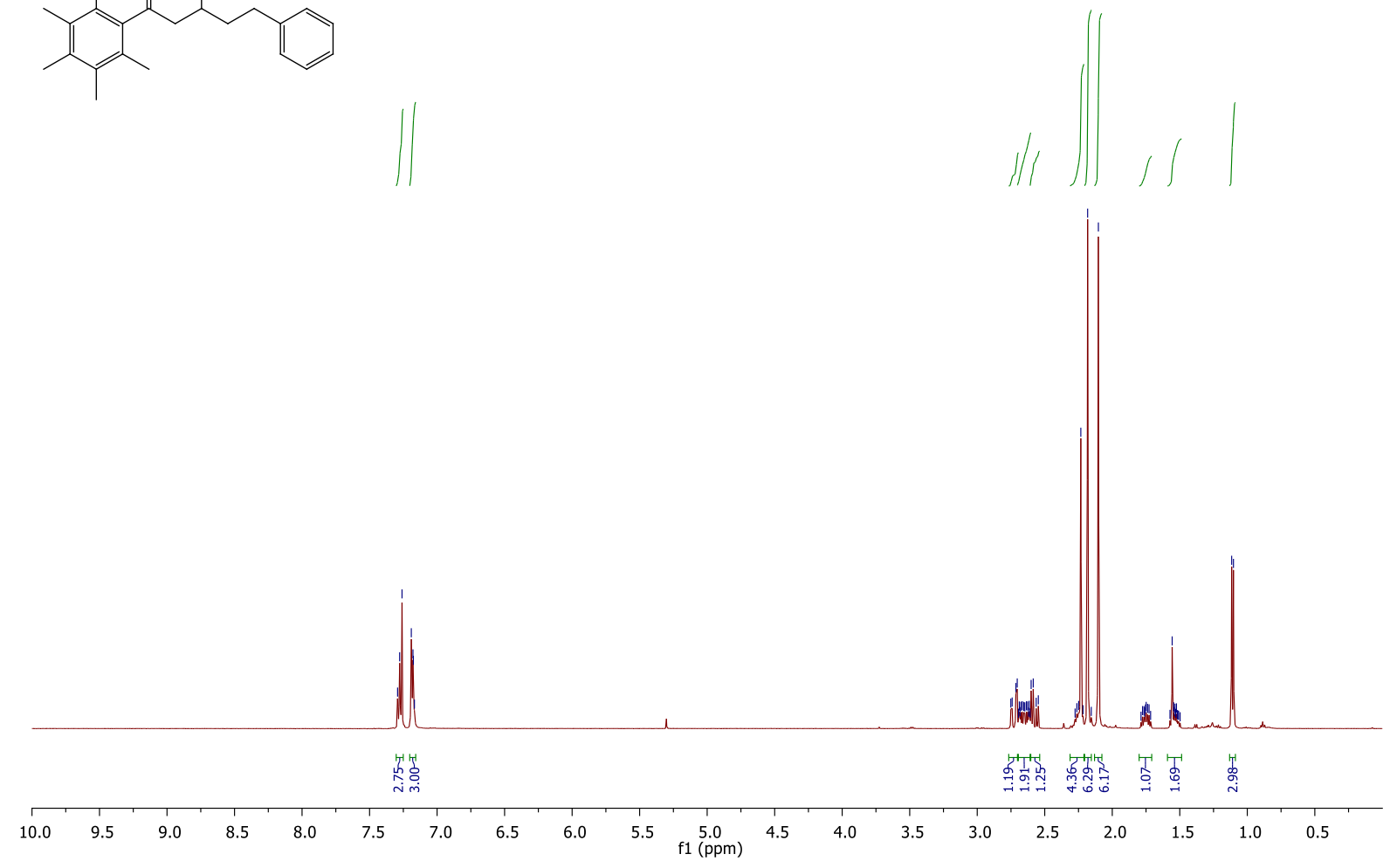

Figure S85: ${ }^{1} \mathrm{H}-\mathrm{NMR}(500 \mathrm{MHz})$ in $\mathrm{CDCl}_{3}$ of $2 \mathrm{~h}$
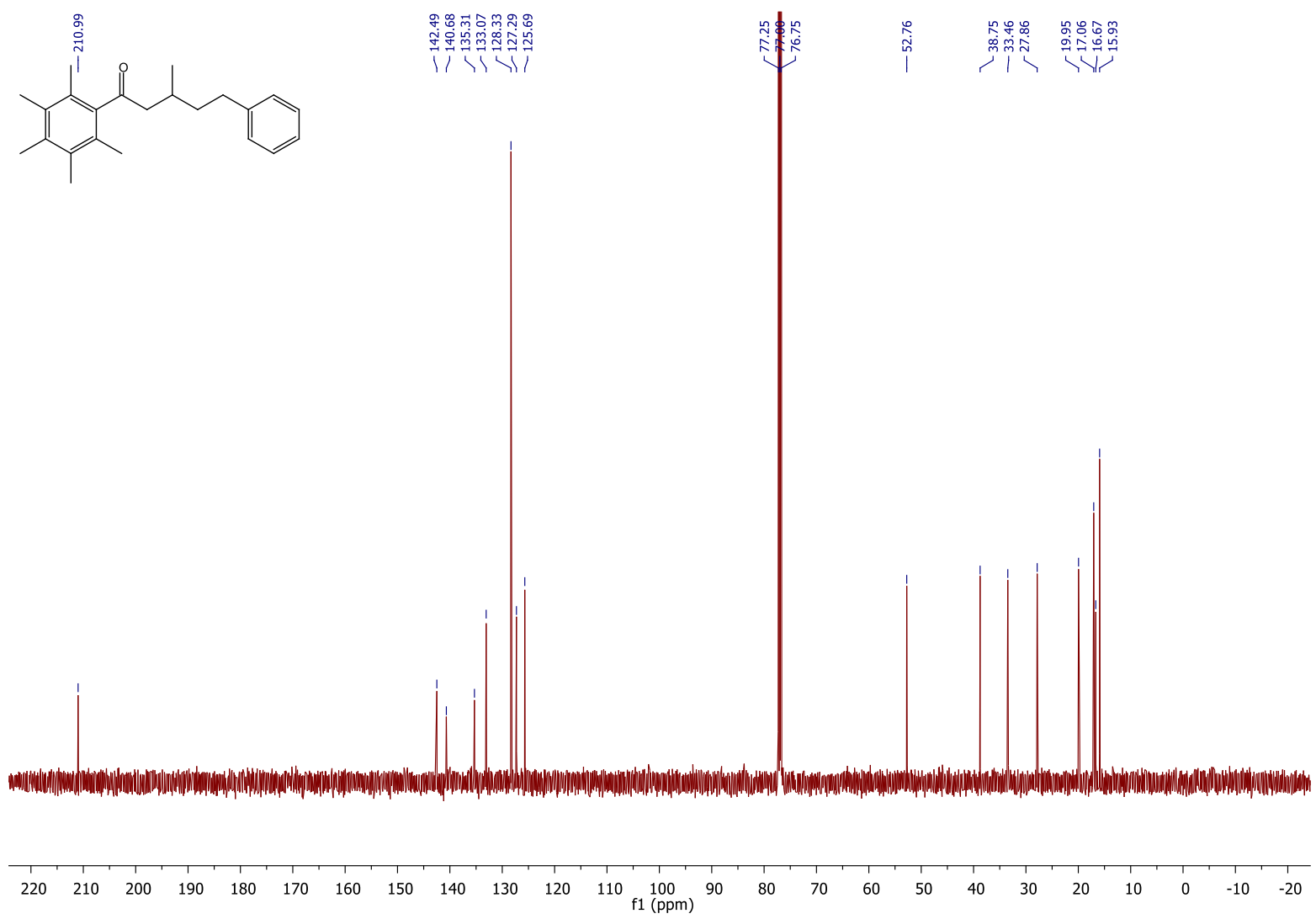

Figure S86: ${ }^{13} \mathrm{C}-\mathrm{NMR}(125 \mathrm{MHz})$ in $\mathrm{CDCl}_{3}$ of $2 \mathrm{~h}$

S-66 


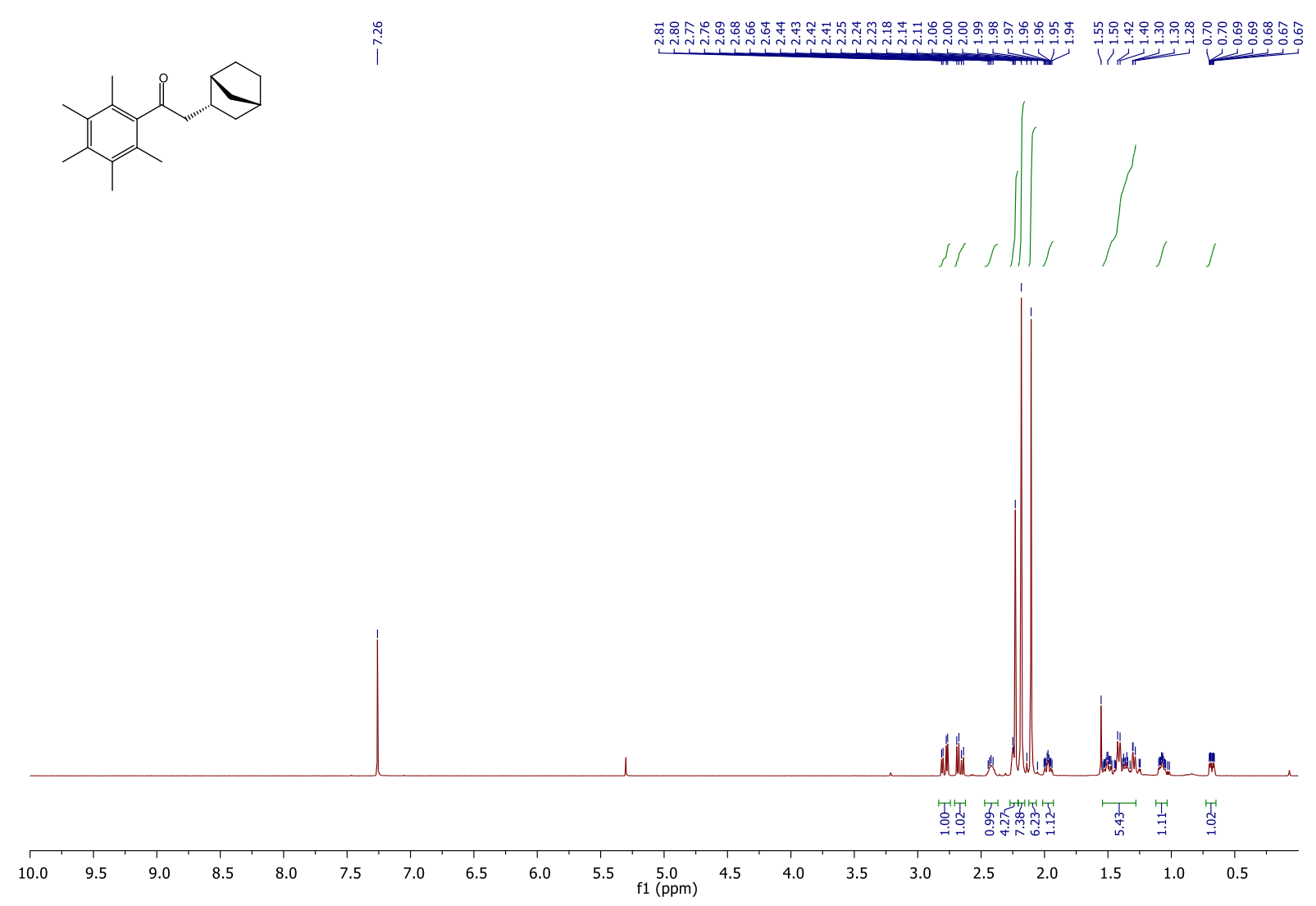

Figure S87: ${ }^{1} \mathrm{H}-\mathrm{NMR}(500 \mathrm{MHz})$ in $\mathrm{CDCl}_{3}$ of $2 \mathrm{i}$

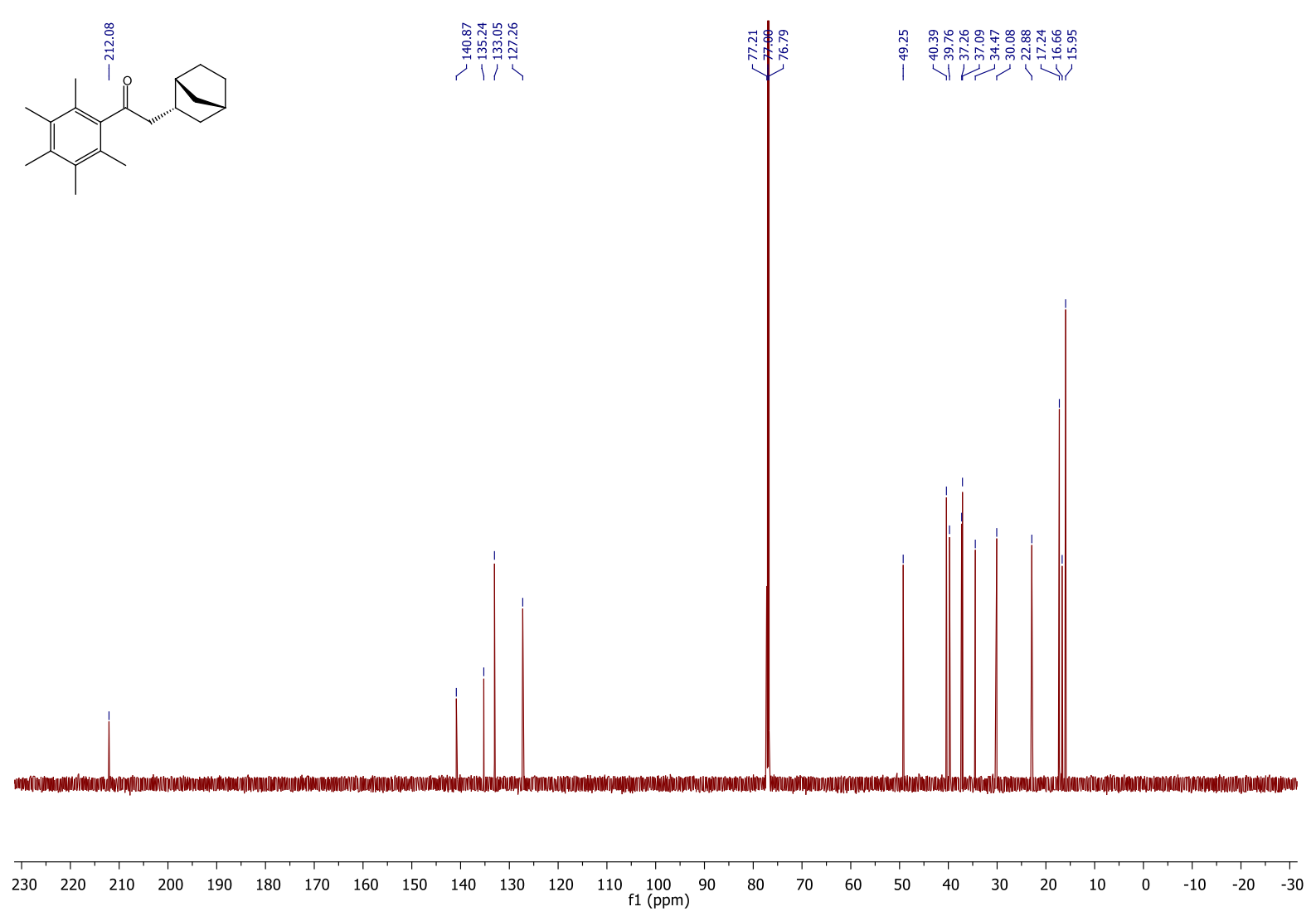

Figure S88: ${ }^{13} \mathrm{C}$-NMR (125 MHz) in $\mathrm{CDCl}_{3}$ of $2 \mathrm{i}$ 


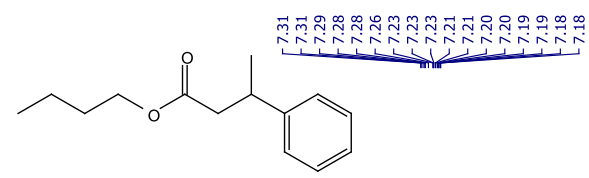

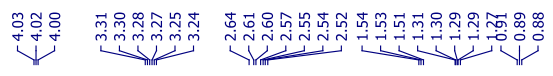
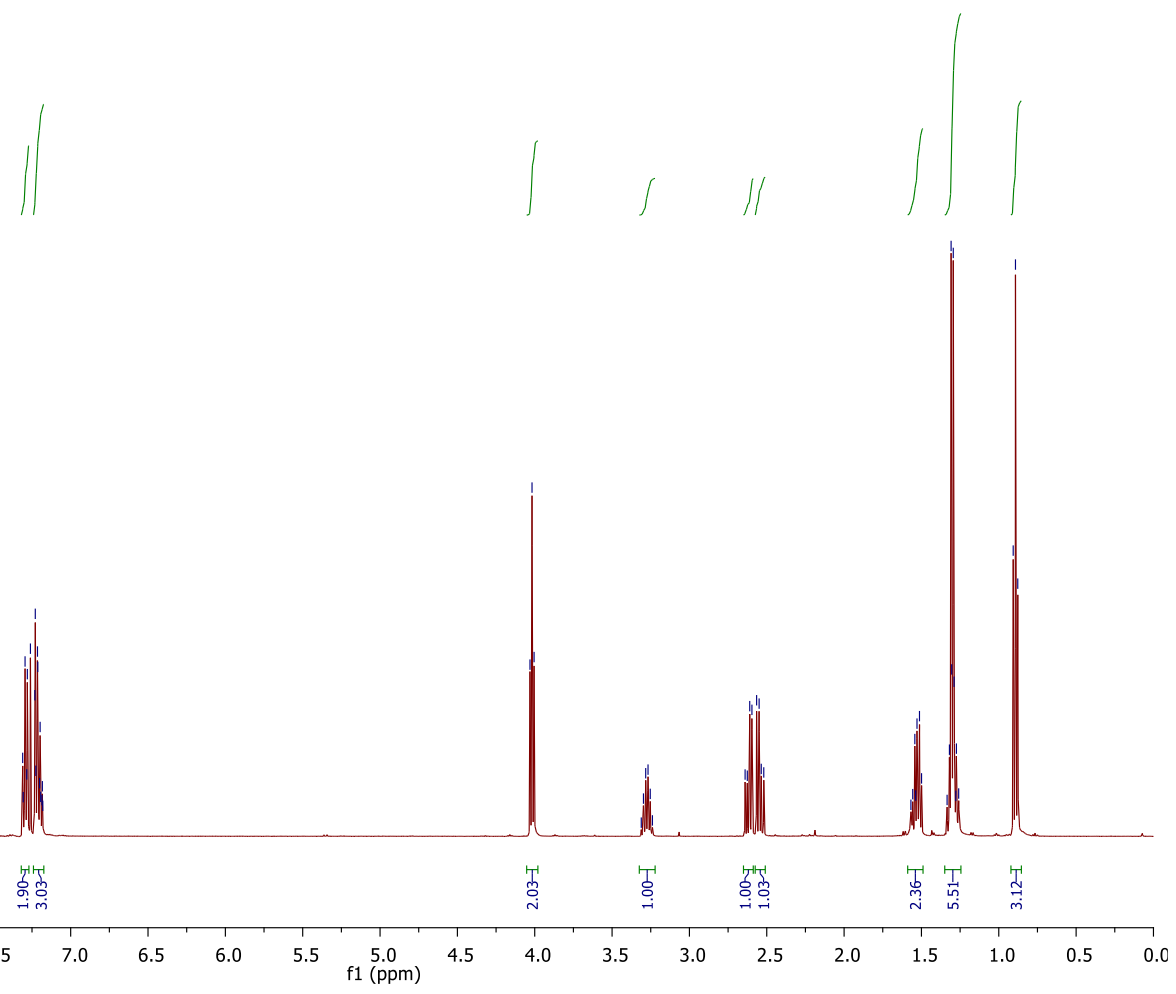

Figure S89: ${ }^{1} \mathrm{H}-\mathrm{NMR}(500 \mathrm{MHz})$ in $\mathrm{CDCl}_{3}$ of $4 \mathrm{a}$
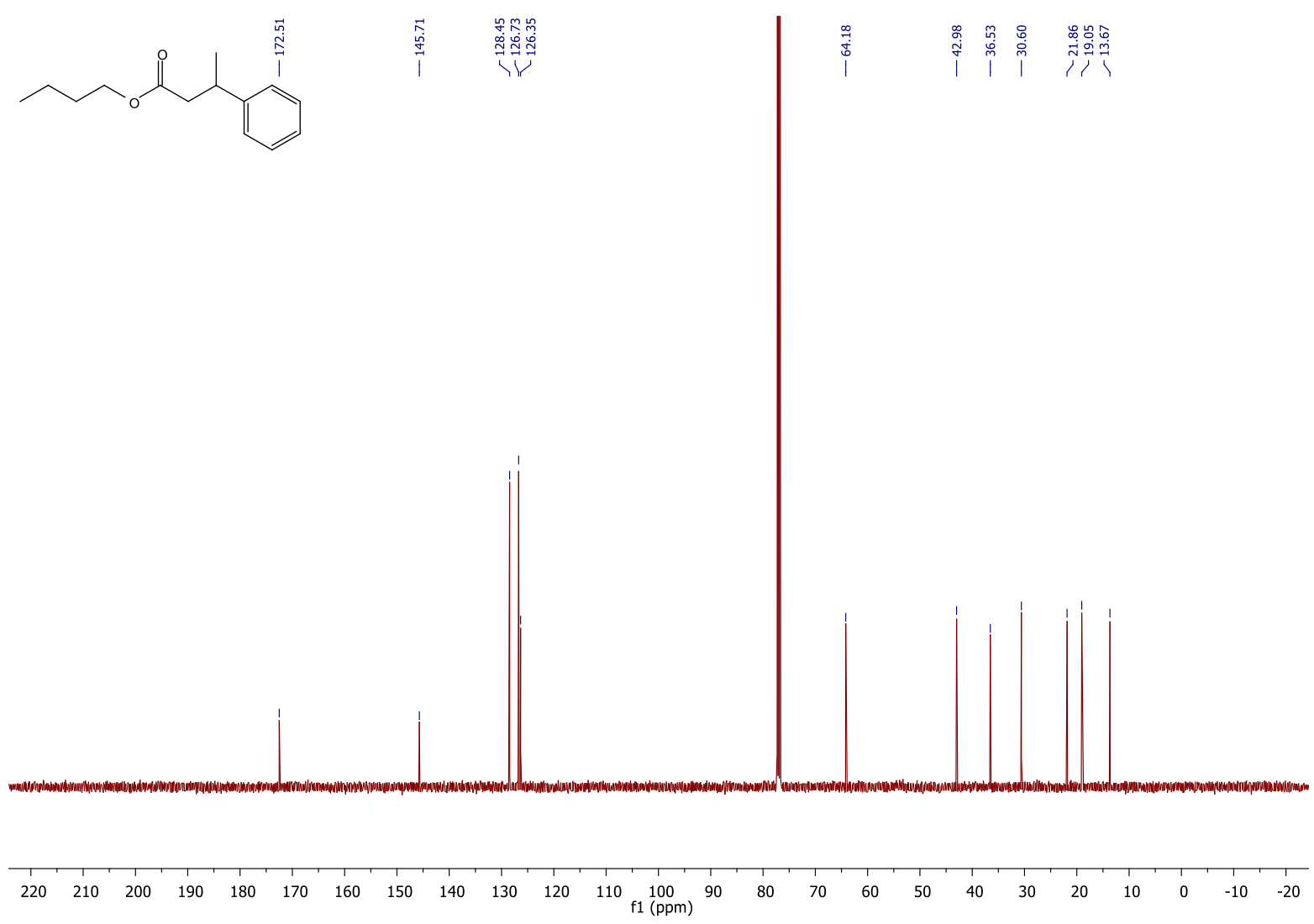

Figure S90: ${ }^{13} \mathrm{C}-\mathrm{NMR}(125 \mathrm{MHz})$ in $\mathrm{CDCl}_{3}$ of $4 \mathrm{a}$

S-68 


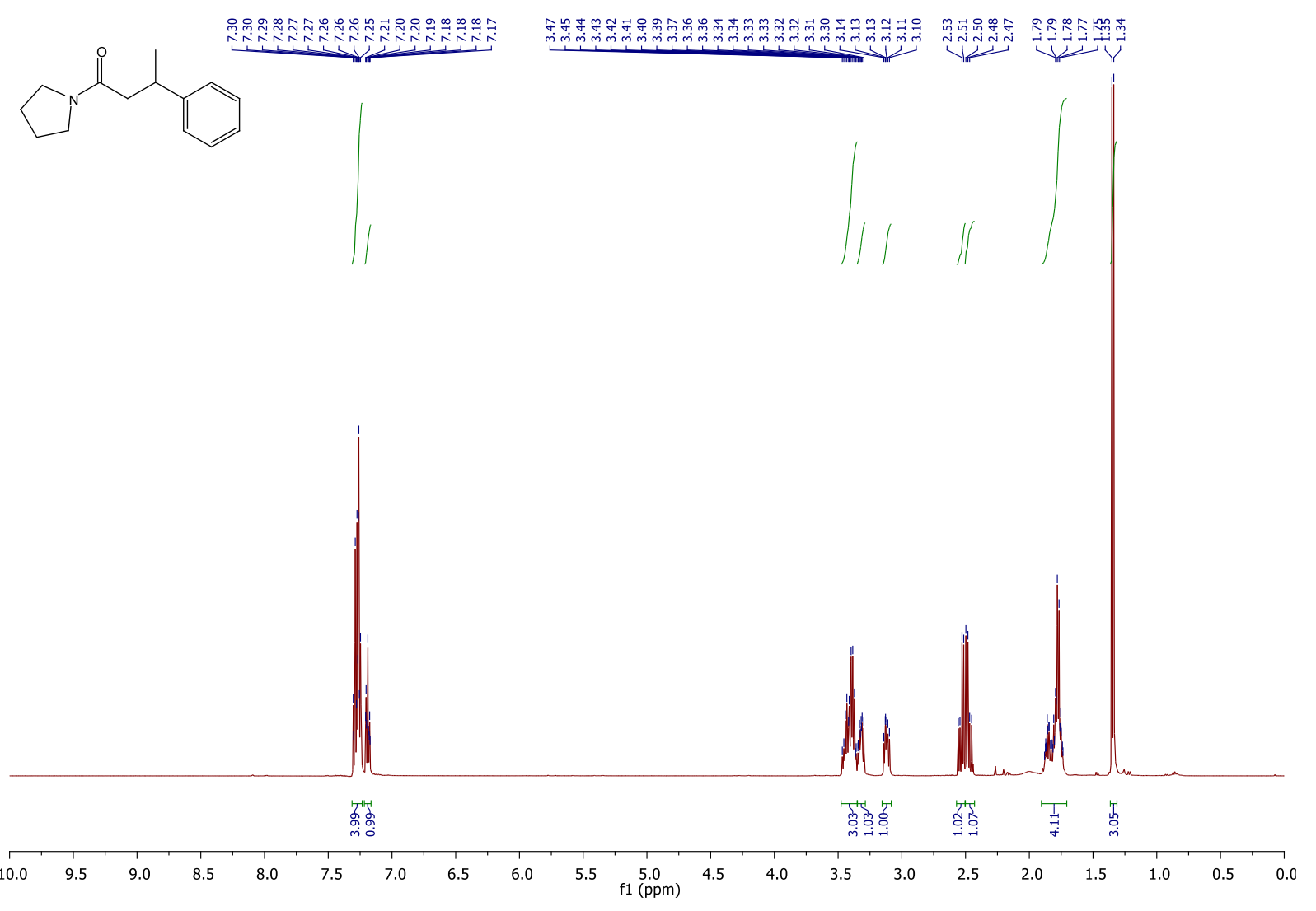

Figure S91: ${ }^{1} \mathrm{H}-\mathrm{NMR}(500 \mathrm{MHz})$ in $\mathrm{CDCl}_{3}$ of $4 \mathrm{~b}$
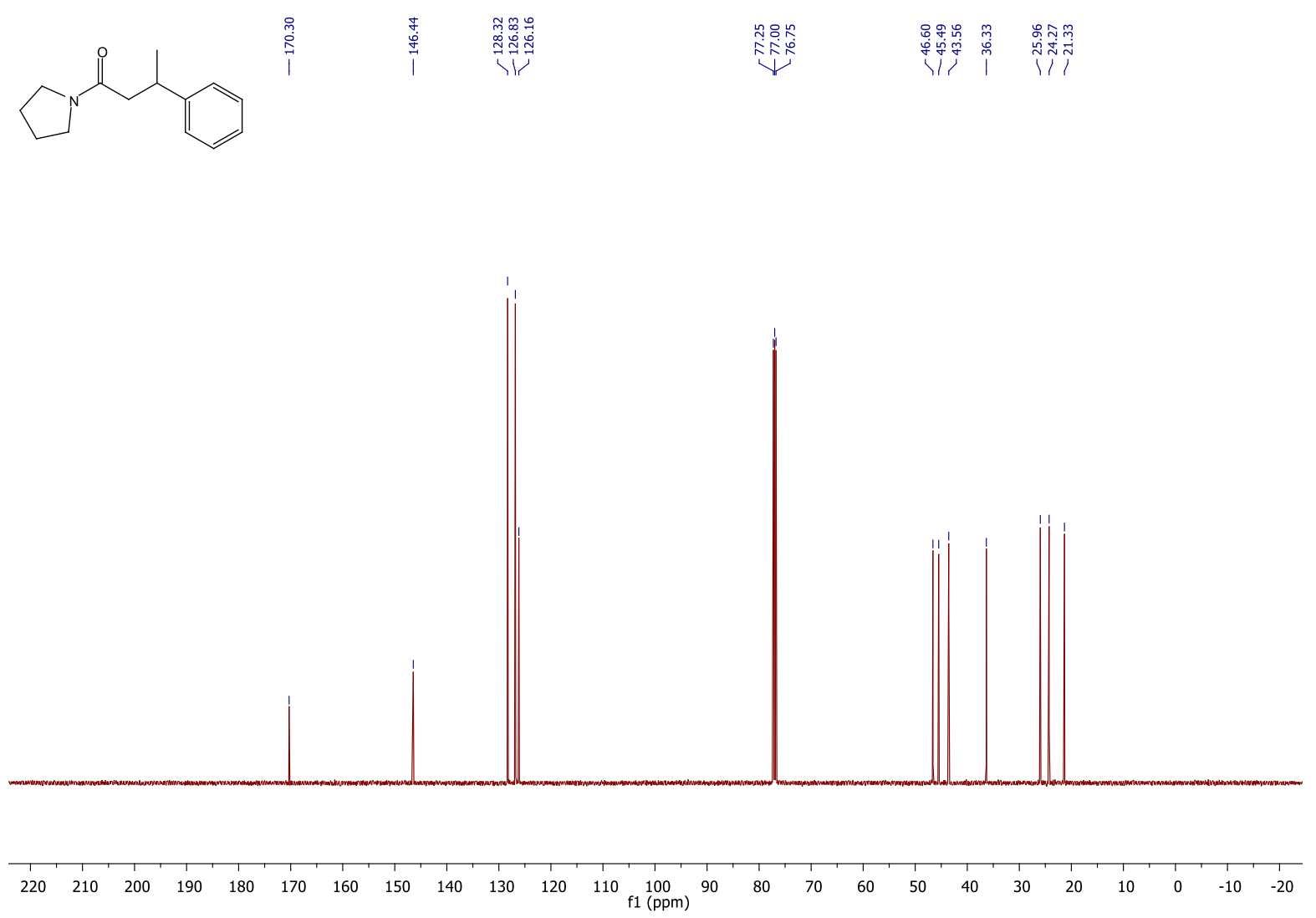

Figure S92: ${ }^{13} \mathrm{C}-\mathrm{NMR}(125 \mathrm{MHz})$ in $\mathrm{CDCl}_{3}$ of $4 \mathrm{~b}$

S-69 


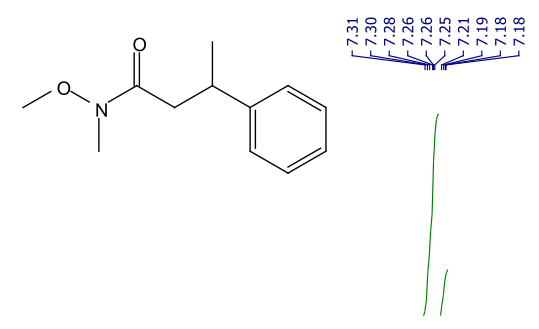

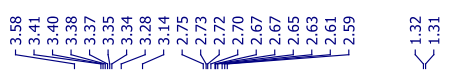

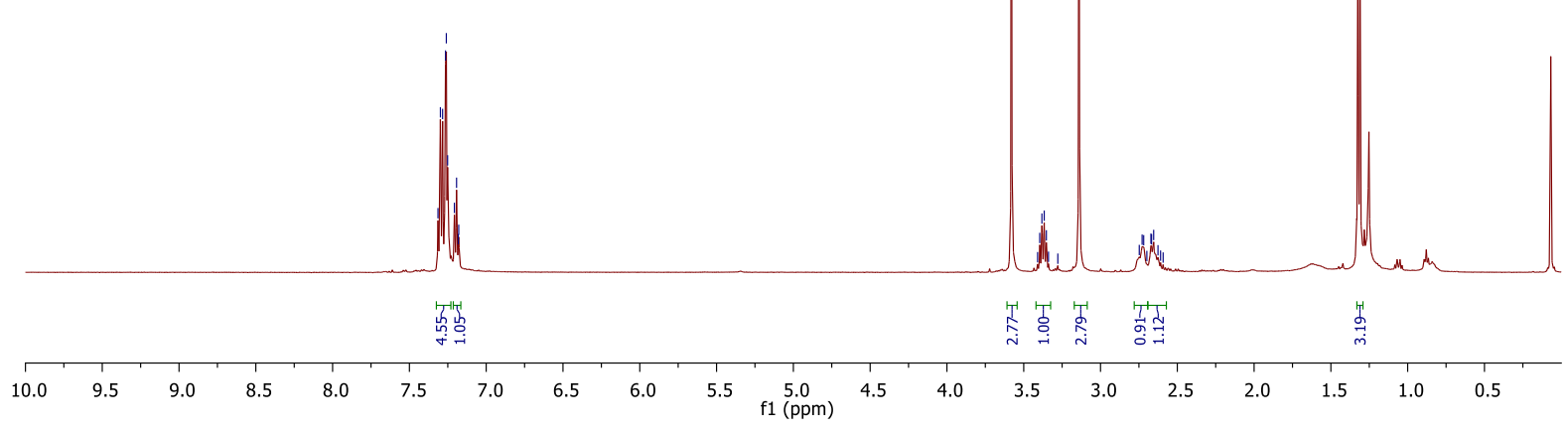

Figure S93: ${ }^{1} \mathrm{H}-\mathrm{NMR}(500 \mathrm{MHz})$ in $\mathrm{CDCl}_{3}$ of $4 \mathrm{C}$
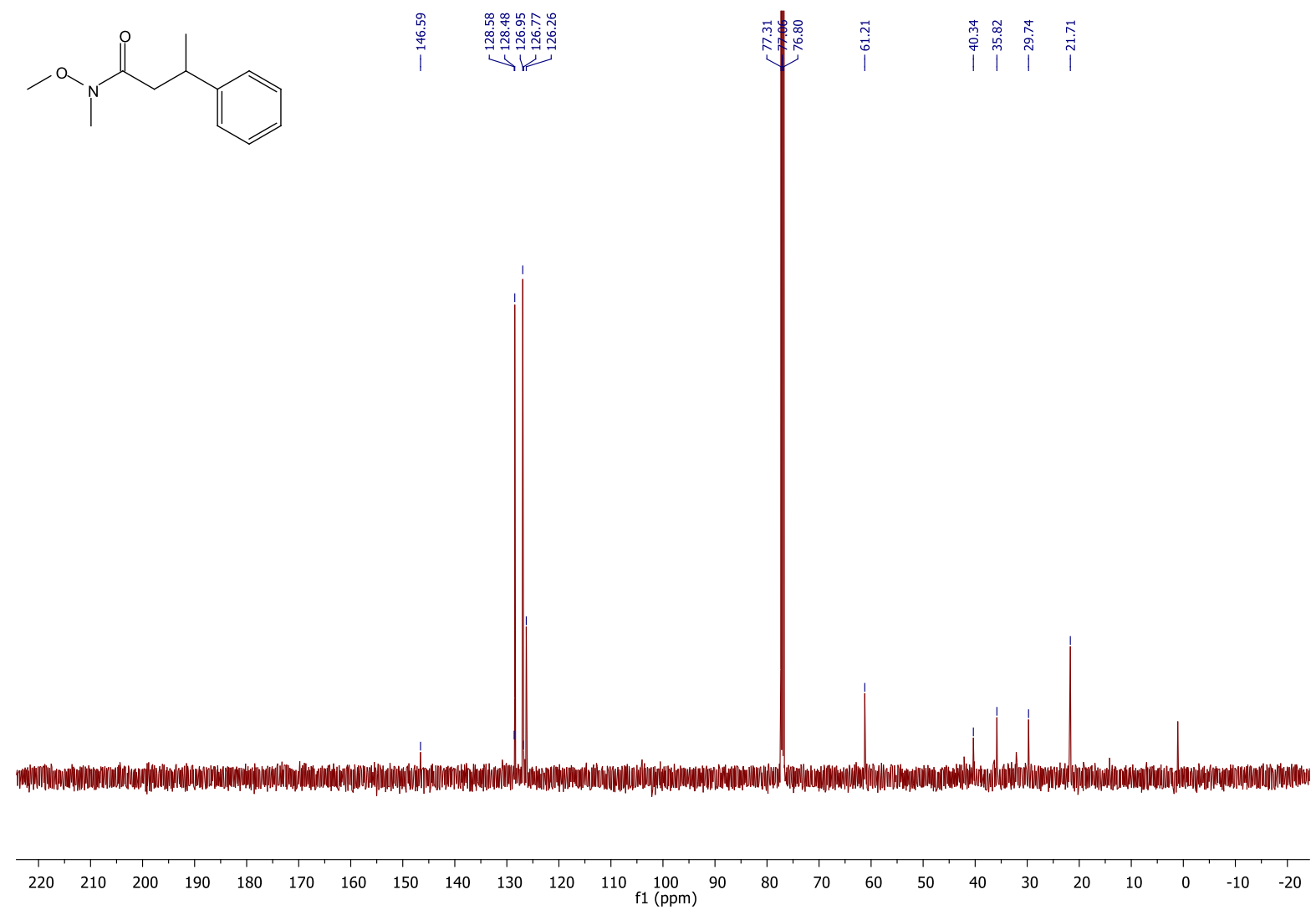

Figure S94: ${ }^{13} \mathrm{C}-\mathrm{NMR}(125 \mathrm{MHz})$ in $\mathrm{CDCl}_{3}$ of $4 \mathrm{C}$

S-70 


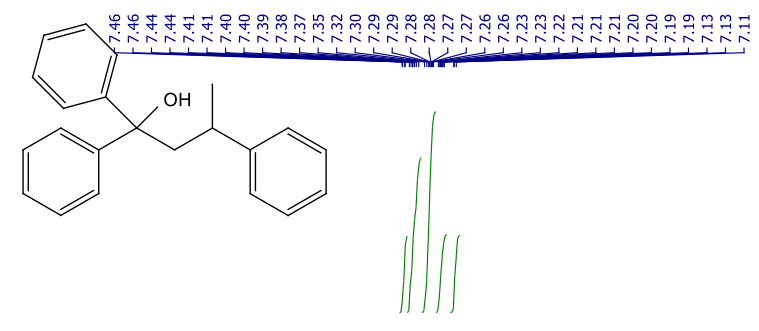

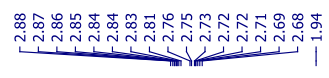

ำ

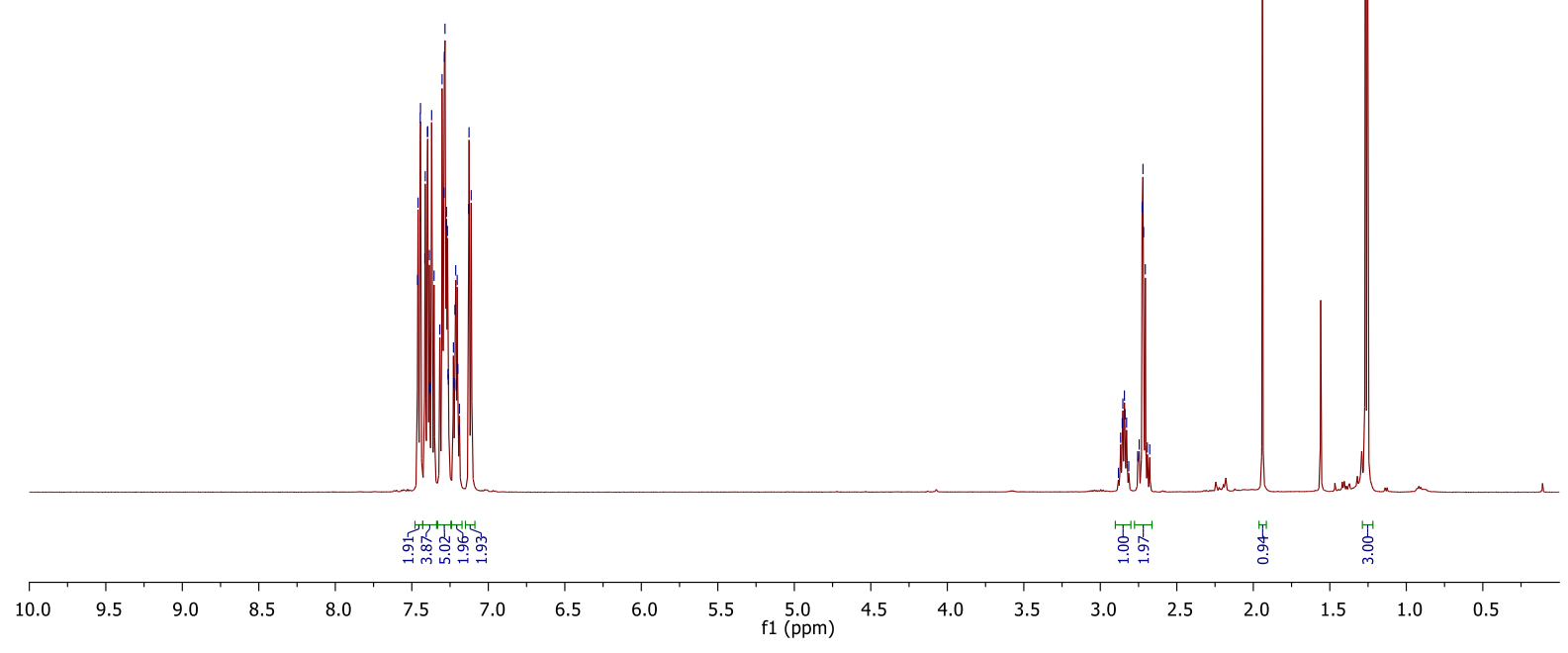

Figure S95: ${ }^{1} \mathrm{H}-\mathrm{NMR}(500 \mathrm{MHz})$ in $\mathrm{CDCl}_{3}$ of $4 \mathrm{~d}$
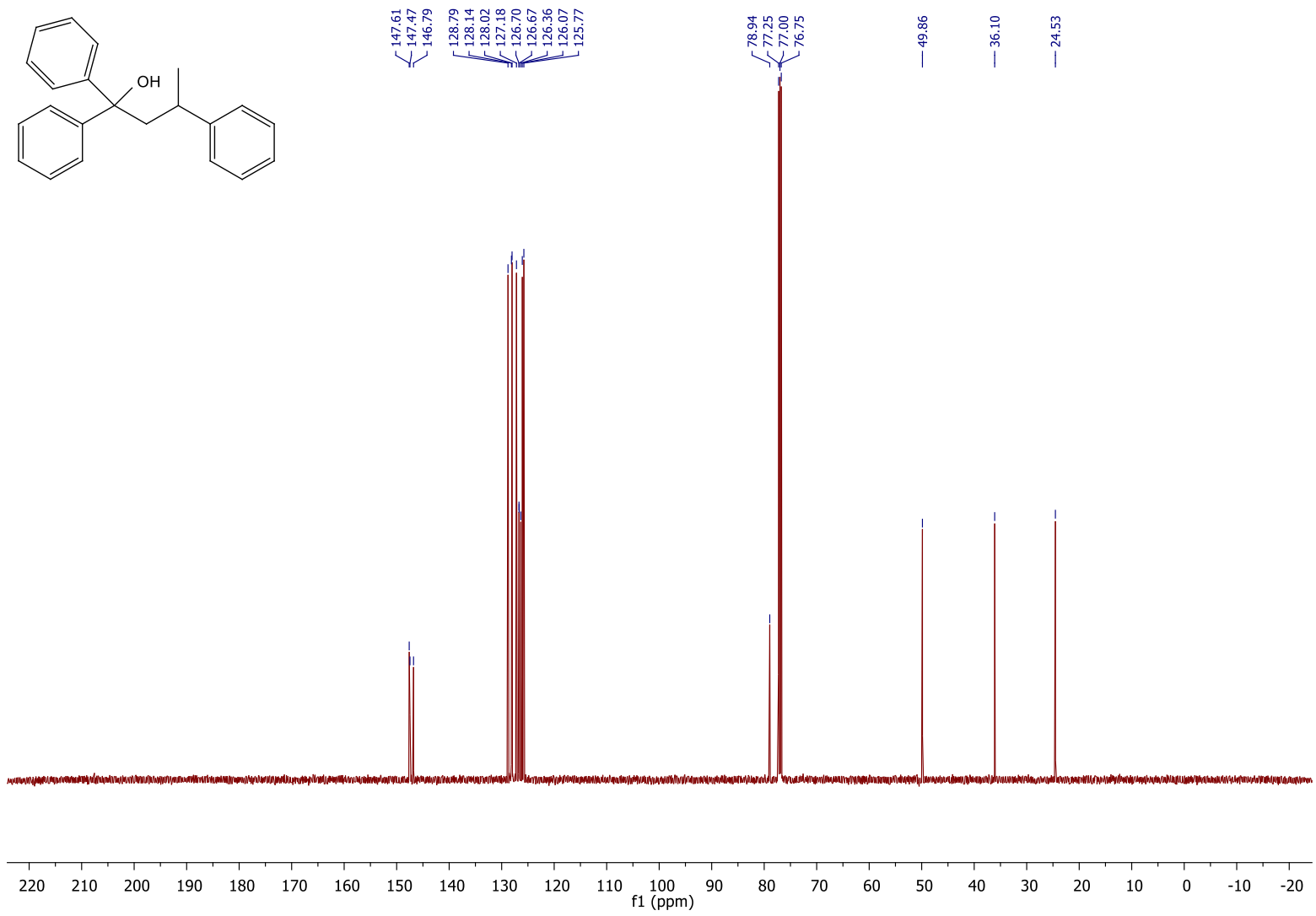

Figure S96: ${ }^{13} \mathrm{C}-\mathrm{NMR}(125 \mathrm{MHz})$ in $\mathrm{CDCl}_{3}$ of $4 \mathrm{~d}$

S-71 


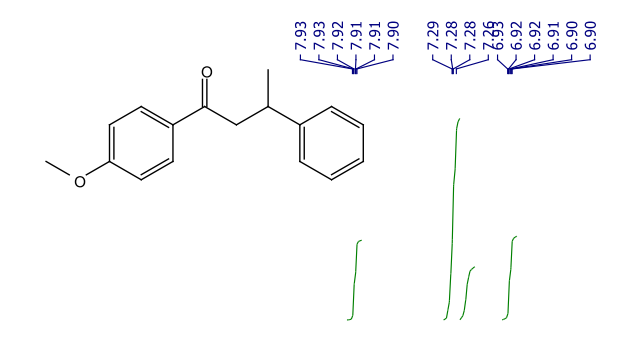

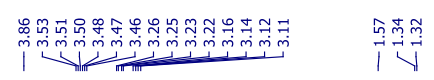

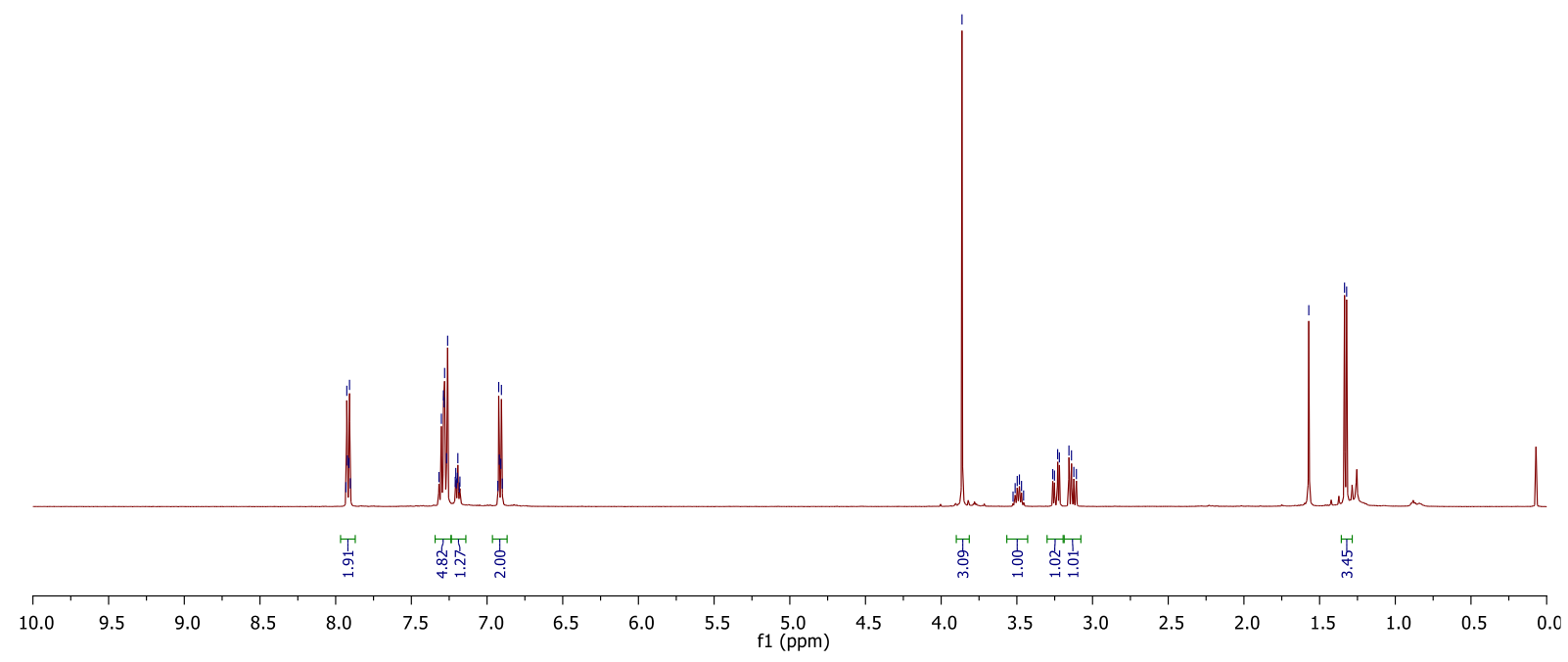

Figure S97: ${ }^{1} \mathrm{H}-\mathrm{NMR}(500 \mathrm{MHz})$ in $\mathrm{CDCl}_{3}$ of $4 \mathrm{e}$
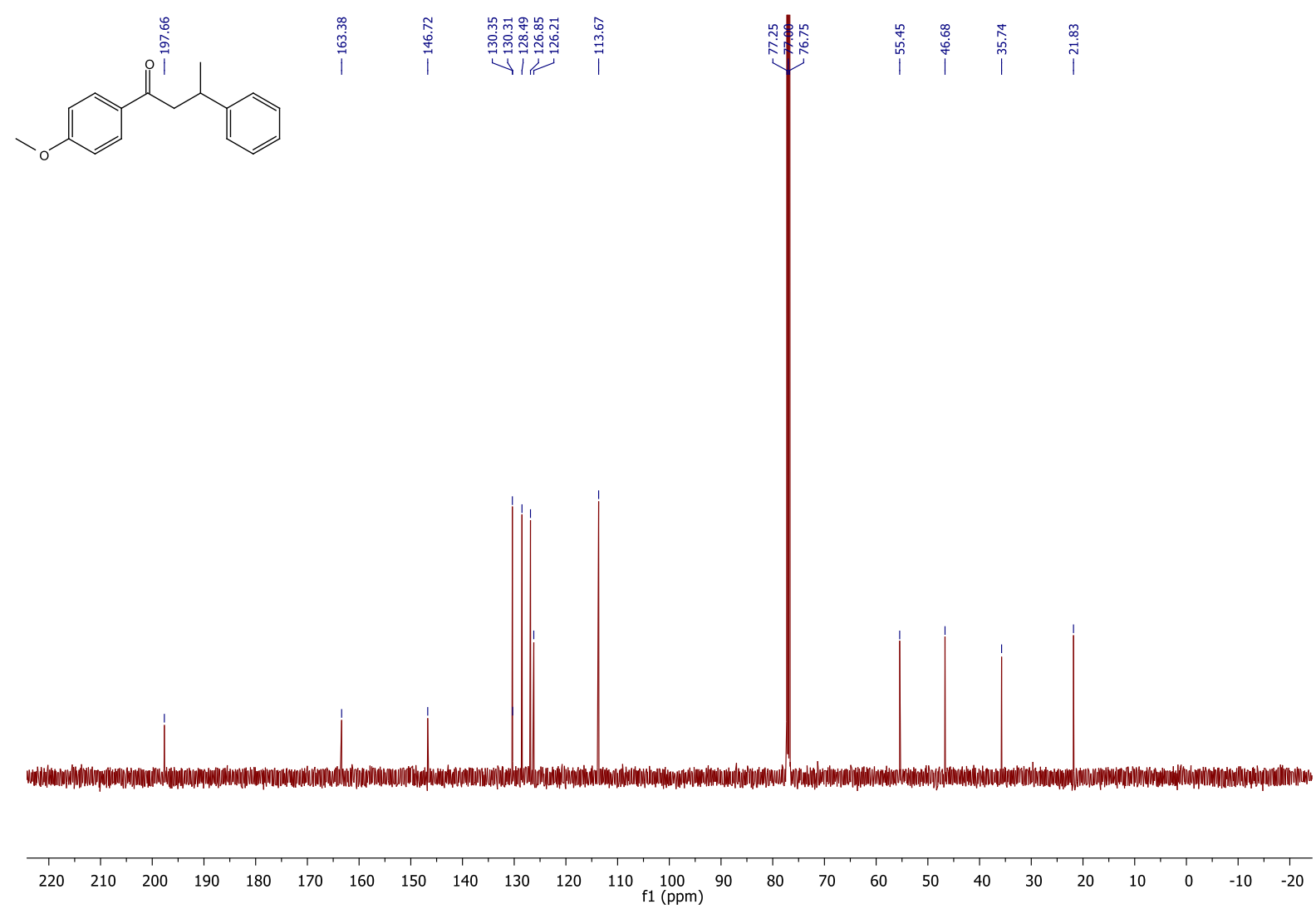

Figure S98: ${ }^{13} \mathrm{C}-\mathrm{NMR}(125 \mathrm{MHz})$ in $\mathrm{CDCl}_{3}$ of $4 \mathrm{e}$ 


\section{Part 7: Crystallographic Data of Compound 2i}

\section{X-Ray Crystal Structure of $2 \mathrm{i}$}<smiles>Cc1c(C)c(C)c(C(=O)C[C@@H]2CC3CCC2C3)c(C)c1C</smiles>

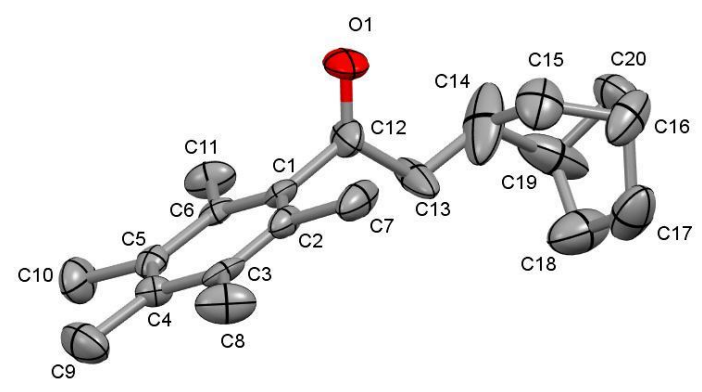

Single crystals of $\mathbf{2} \mathbf{i}$ suitable for $\mathbf{X}$-ray crystallographic analysis were obtained by slow evaporation of $\mathrm{MeOH}$ solution. X-ray diffraction experiments for monocrystal of $\mathbf{X}$ were performed at $150 \mathrm{~K}$ with graphite-monochromatized Mo $\mathrm{K}_{\alpha}$ radiation $(\lambda=0.71073 \AA)$ on a Bruker D8Quest diffractometer. Formula $\mathrm{C}_{20} \mathrm{H}_{28} \mathrm{O}$, formula weight 284.42 , crystal system orthorhombic, space group Pna2 $1, a=11.040(4) \AA, b=5.989(2) \AA, c=24.915(7) \AA, \alpha=\beta=\gamma$ $=90^{\circ}, V=1647.4(9) \AA^{3}, Z=4$, Calcd for density $=1.147 \mathrm{~g} / \mathrm{cm}^{3}, \mu=0.068 \mathrm{~mm}^{-1}, R_{\text {int }}=0.1474$, $\mathrm{R}\left[F^{2}>2 \sigma\left(F^{2}\right)\right]=0.1244, \mathrm{wR}\left(F^{2}\right)=0.2998$. Reflections measured $=9583$, independent reflections $=4036$, observed reflections $=1434$. CCDC number $: 1982548$. Program $(s)$ used to solve structure: SHELXT 2014/5. Program(s) used to refine structure: SHELXL-2018/3. Software used to prepare material for publication: SHELXTL. 